BRUNO CALDAS DE SOUZA

\title{
TOPOLOGY OPTIMIZATION OF FLUID DIODES BASED ON INTEGER LINEAR PROGRAMMING
}


BRUNO CALDAS DE SOUZA

\section{TOPOLOGY OPTIMIZATION OF FLUID DIODES BASED ON INTEGER LINEAR PROGRAMMING}

Doctoral thesis submitted to the Escola Politécnica of University of São Paulo in partial fulfillment of the requirements for the Doctor of Science degree . 
BRUNO CALDAS DE SOUZA

\title{
TOPOLOGY OPTIMIZATION OF FLUID DIODES BASED ON INTEGER LINEAR PROGRAMMING
}

\author{
Revised version \\ (Versão corrigida)
}

Doctoral thesis submitted to the Escola Politécnica of University of São Paulo in partial fulfillment of the requirements for the Doctor of Science degree .

Area of Concentration:

Engenharia de Controle e Automação Mecânica

Advisor:

Prof. Dr. Emílio Carlos Nelli Silva 
Autorizo a reprodução e divulgação total ou parcial deste trabalho, por qualquer meio convencional ou eletrônico, para fins de estudo e pesquisa, desde que citada a fonte.

Este exemplar foi revisado e corrigido em relação à versão original, sob responsabilidade única do autor e com a anuência de seu orientador.

São Paulo, 15 de Maio de 2021

Assinatura do autor: Bruno Caldas de bouza.

Assinatura do orientador:

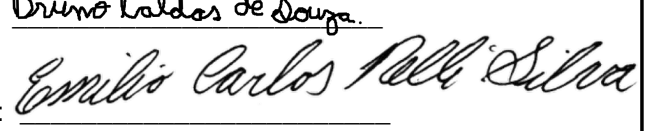

\section{Catalogação-na-publicação}

\section{SOUZA, BRUNO CALDAS DE}

Topology Optimization of Fluid Diodes based on Integer Linear

Programming / B. C. D. SOUZA -- versão corr. -- São Paulo, 2021. $145 \mathrm{p}$.

Tese (Doutorado) - Escola Politécnica da Universidade de São Paulo. Departamento de Engenharia Mecatrônica e de Sistemas Mecânicos.

1.1.Métodos Topológicos (Otimização) 2.Dinâmica dos Fluidos (Simulação) 3.Diodos Fluídicos 4.Juntas Labrintos 5.Método dos Elementos Finitos I.Universidade de São Paulo. Escola Politécnica. Departamento de Engenharia Mecatrônica e de Sistemas Mecânicos II.t. 


\section{ACKNOWLEDGMENTS}

Firstly, I would like to express my sincere gratitude to my advisor Prof. Dr. Emílio Carlos Nelli Silva for the continuous support of my DSc study and related research, for his patience, motivation, and immense knowledge. His guidance helped me in all the time of research and writing of this thesis.

I would like to thank CAPES and all the RCGI support including BG-Shell partnership, which made feasible the start and continuance of my research.

Also, I thank my research friends at University of Sao Paulo for all support and contribution to this work.

Finalmente gostaria de agradecer à minha família, em especial a minha mãe Rosangela e minha esposa Camila pelo amor incondicional, além do imprescindível apoio psicológico e financeiro dado durante todo o trabalho. Sem essas mulheres guerreiras nada disto seria possível. 


\section{RESUMO}

Diodos fluídicos são dispositivos que inibem o fluxo de fluido de um ambiente para outro, porém permitindo que em alguns casos ocorra o sentido oposto. Um exemplo bem conhecido é a válvula Tesla. Existe um tipo particular de diodo fluídico usado para minimizar vazamentos em turbinas e compressores chamados selos labirinto, cuja otimização pode trazer grandes melhorias relacionadas às emissões de $\mathrm{CO}_{2}$ e $\mathrm{CH}_{4}$. A otimização topológica é uma ferramenta de otimização poderosa, que já foi aplicada para projetar alguns diodos fluidos planos 2D. Assim, com esta questão ambiental em mente e a fim de encontrar novos selos labirintos eficientes, o escopo deste trabalho é desenvolver uma nova formulação de otimização topológica para obter projetos inovadores e não intuitivos de diodos fluídicos focado em selos labirintos. Como primeira contribuição, a formulação de otimização topológica proposta neste trabalho baseia-se na programação linear inteira. Na atual implementação de otimização de topológica para fluidos considerando métodos de densidade, existem essencialmente dois problemas. Primeiramente, a escala de cinza no resultado dificulta a identificação do contorno da malha do fluido, o que pode ser um problema em algumas aplicações também durante o processo de otimização. Em segundo lugar, mesmo para problemas de projeto de fluxo de Reynolds baixo, um esquema de continuação dos parâmetros de penalização do modelo de material é necessário para evitar a escala de cinza e para obter fronteiras sólido/fluido claras. Este trabalho propõe uma nova metodologia que resolve esses dois problemas, ou seja, evita a escala de cinza e obtém fronteiras de sólido e fluido mais claras. Já no projeto do selo labirinto, o mesmo apresenta partes fixas (estator) e rotacionais (eixo), assim como segunda contribuição, um algoritmo de classificação é implementado para identificar partes pertencentes ao eixo e ao sólido e desta forma aplicar condições de contorno rotacionais a cada sólido que surge durante a otimização topológica. Finalmente, uma função objetivo considerando alguma interação fluido-estrutura é definida para evitar o surgimento de ilhas, que são muito comuns neste tipo de projeto, porém não são aceitáveis em projetos axissimétricos de selos labirinto. Assim a função multiobjetivo definida contém termos de energia de dissipação, vorticidade e funções estruturais. Com relação à geometria do selo labirinto, o problema é modelado com um modelo de elemento finito girante 2D. É descrito o cálculo de sensibilidades e a linearização do problema para otimização em variáveis inteiras. A implementação numérica é feita em Python com o auxílio de bibliotecas de elementos finitos (FEniCS) para calcular o problema direto e adjunto. A biblioteca de otimização proprietária da IBM (CPlex) é usada como algoritmo de otimização. Como resultados são apresentados projetos de selo labirinto otimizados obtidos de acordo com o objetivo do projeto, variando a rotação, padrões de vazamento e razão de aspecto do domínio do projeto.

Palavras-Chave - Diodos Fluídicos, Otimização Topológica, Junta Labirinto, TOBS, método de elementos finitos, Fenics. 


\section{ABSTRACT}

Fluid diodes are devices that inhibit the fluid to flow from one environment to another and allowing in some cases the opposite direction to occur. A well-known example is the Tesla valve. There is a particular type of fluid diode used to minimize leakage in turbines and compressors called labyrinth seals, which optimization can bring major improvement related to $\mathrm{CO}_{2}$ and $\mathrm{CH}_{4}$ emissions. Topology optimization is a powerful tool, which has already been applied to design some plane $2 \mathrm{D}$ fluid diodes. So, with this environmental issue in mind and in order to find new efficient labyrinth seals, the scope of this work is to develop a new formulation of topology optimization to obtain innovative and non-intuitive designs of fluid diodes focused on labyrinth seals. As a first contribution, the formulation of topology optimization proposed in this work is based on the integer linear programming. In the current implementation of topology optimization for fluids considering density methods, there are essentially two problems. First, the gray scale in the result makes it difficult to identify the fluid mesh outline, which can be a problem in some applications and also during the optimization process. Second, even for low Reynolds flow design problems, a continuation scheme of the material model penalty parameters is necessary to avoid gray scale and to obtain clear solid/fluid boundaries. This work proposes a new methodology that solves these two problems, that is, it avoids gray scale and obtains clearer solid and fluid boundaries. In the labyrinth seal design, on the other hand, it presents fixed parts (stator) and rotational parts (axis), as well as a second contribution, a classification algorithm is implemented to identify parts belonging to the shaft and, thus, apply rotational boundary conditions to every solid that appears during topolgy optimization. Finally, an objective function considering some fluid-structure interaction is defined to prevent the appearance of islands, which are very common in this type of project, however, they are not acceptable in axysymmetric designs of labyrinth seals. Thus the defined multi-objective function contains terms of dissipation energy, vorticity and structural functions. Regarding the geometry of the labyrinth seal, the problem is modeled with a 2D swirl finite element model. The calculation of sensitivities and the linearization of the problem for optimization in integer variables is described. Numerical implementation is done in Python with the aid of finite element libraries (FEniCS) to calculate the direct and adjoint problem. The IBM proprietary optimization library (CPlex) is used as an optimization algorithm. As results, optimized labyrinth seal designs obtained according to the project objective are presented, varying the rotation, leakage patterns and aspect ratio of the project domain.

Keywords - Fluid Diodes, Topology Optimization, Labyrinth Seal, TOBS, finite element method, Fenics. 


\section{LIST OF FIGURES}

1 Possible fluid diodes devices. . . . . . . . . . . . . . . . . . . . . . . . 19

2 Pulsating heating pipes. . . . . . . . . . . . . . . 20

3 Labyrinth Seals in rotating environment. . . . . . . . . . . . . . . 20

4 Actual Application of a Labyrinth Seal [LIN et al., 2014] . . . . . . . . . . . 21

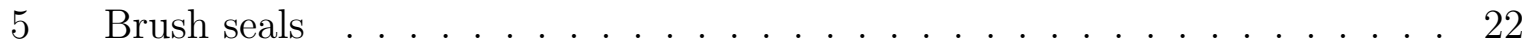

6 Schematic representation of a generic stepped labyrinth seal, depicting two cycles of a seal [VAKILI et al., 2006] . . . . . . . . . . . . . . . . 23

7 Natural Gas Reinjection. . . . . . . . . . . . . . . . . . . . . 24

8 Mechanical Contact Face Seal (MFS). . . . . . . . . . . . . . . . . 25

9 Liquid Film Seal (LFS). . . . . . . . . . . . . . . . . . . . . 26

10 Dry Gas Seal (DGS). . . . . . . . . . . . . . . . . 27

11 Possible parameters for generic seals. . . . . . . . . . . . . . . . . . 27

12 Possible LS geometries: (a)Interlocking (b)Traditional Stepped. . . . . . . 28

13 Optimization of labyrinth seal based on intuition. . . . . . . . . . . . . . 29

14 Illustration of the effect on the flow due to the rotation of an axis. . . . . . 30

15 Possible types of fluid diodes optimization. . . . . . . . . . . . . . . 31

16 Topology optimization procedure for a labyrinth seal. . . . . . . . . . . . 32

17 Incompressible flows according to Mach Number. . . . . . . . . . . . . . 39

18 Coordinates System chosen to model the 2.5D System. . . . . . . . . . . . 41

19 Taylor Hood Element and its interpolation. . . . . . . . . . . . . . . . . . 42

20 Triangular Element with coordinate frame. . . . . . . . . . . . . . 43

21 Plot of the relationship between the $\mathrm{q}, \alpha$ and $\kappa$ parameters based on [BORRVALL; PETERSSON, 2003]. . . . . . . . . . . . . . . . . 46 
22 Result of maximizing energy dissipation in a labyrinth seal (black: solid, white: fluid). . . . . . . . . . . . . . . . . . . . 48

23 Fluid flow load cases. . . . . . . . . . . . . . . . . . . . . . . . 48

24 Issues with the proposed problem with simple diodicity. . . . . . . . . . . . 49

25 Scheme showing how forces and boundary conditions are applied on structures with $\mathbf{u}$ as velocity with parabolic profile on inlet with magnitude according to a Reynods number value. . . . . . . . . . . . . . . . . . 51

26 Results when considering structural terms or not. . . . . . . . . . . . . 52

27 Elements $i$ of a Neighboorhood $\left(N^{e}\right)$ inside a distance of $r_{\min }$ to an element $e .53$

28 Solid position and rotational speed. . . . . . . . . . . . . . . . 55

29 Delaunay algorithm to determine if a point belongs to a circle. . . . . . . . 56

30 Classification algorithm detecting geometries. . . . . . . . . . . . 56

31 Rotation selection using classification for new geometries during TO process. 57

32 Classification algorithm used to design fluid diodes. . . . . . . . . . . . . 57

33 Overview of Fenics application. . . . . . . . . . . . . 63

34 Programming scheme used in this work. . . . . . . . . . . . . . 64

35 Flowchart of the Topology Optimization procedure with the TOBS Method. 65

36 Detailed implementation of both forward and adjoint problem of Fenics block in Figure $35 . \quad$. . . . . . . . . . . . . . . . . . . . . . . . 65

37 Design domain for the classic Stokes problem with $\mathbf{u}$ according to $R e$ value (spatial dimensions in $\mathrm{m}) . \ldots$. . . . . . . . . . . . . . . . . . 67

38 Mesh of the Stokes Problem with regular 9600 elements. . . . . . . . . . 67

39 Topology optimization Stokes results for $\delta=1.5 \ldots \ldots \ldots$. . . . . . 67

40 Objective function convergence for Stokes classic problem $(\delta=1.5) \ldots$. . . 68

41 Topology optimization results evolution during optimization. . . . . . . . . 69

42 Comparison between methods. . . . . . . . . . . . . . . . . 69

43 Comparison between methods during optimization process. . . . . . . . . . 69

44 Jagged contour caused by the proposed method in some case studies. . . . 70 
45 Results of the mesh independence test. . . . . . . . . . . . . . . . 71

46 Objective function convergence of the mesh independence test. . . . . . . . 72

47 Results with different $\kappa_{\max }$ values at iteration $160 \ldots \ldots \ldots \ldots$

48 Topology optimization Stokes results for $\delta=1.0 \ldots \ldots \ldots \ldots \ldots$

49 Objective function convergence for Stokes classic problem $(\delta=1.0)$ with continuation of $\kappa_{\max } \ldots \ldots \ldots \ldots \ldots \ldots$

50 Results of the influence of $q$ values in the binary optimization. . . . . . . . 75

51 Results evolution during optimization for different $\varepsilon$ values. . . . . . . . . . 76

52 Objective function convergence curves for different $\varepsilon$ values. . . . . . . . . . 77

53 Design domain for a Navier-Stokes problem with $\mathbf{u}$ according to $R e$ value (spatial dimensions in $\mathrm{m}) . \ldots \ldots . \ldots 78$

54 Finite element meshes for the Navier-Stokes problem. . . . . . . . . . . . 78

55 Topology optimization for Navier-Stokes example. . . . . . . . . . . . . . 79

56 Results evolution during optimization. . . . . . . . . . . . . . . . 79

57 Objective function convergence for the Navier-Stokes problem considering the design domain with a central squared hole $(\delta=1.5)$. . . . . . . . 80

58 Topology optimization results for different Re number. . . . . . . . . . . 81

59 3d design domain with $\mathbf{u}$ according to $R e$ value. . . . . . . . . . . . . . 82

60 Mesh discretization of the 3D problem (243000 elements) . . . . . . . . . . 82

61 Results evolution during optimization in the 3D design domain. . . . . . . 83

62 Convergence plot of the objective function in the 3D design domain. . . . . 84

63 Design domain of the fluid diode problem with $\mathbf{u}$ according to Re value. . . 84

64 Mesh discretization of the fluid diode problem (7200 elements). . . . . . . . 85

65 Results evolution during optimization using traditional density method. . . 86

66 Results evolution during optimization using binary approach. . . . . . . . . 87

67 Fluid flow simulation of the optimized result with material model. . . . . . 87

68 Fluid flow simulation of extracted geometry. . . . . . . . . . . . . 88 
69 Fluid flow simulation. . . . . . . . . . . . . . . . . . . . . 88

70 Fluid flow simulation for ratio aspect of $4: 3 \ldots \ldots \ldots$. . . . . . . 88

71 Fluid flow simulation.for ratio aspect of $6: 3 \ldots \ldots \ldots$. . . . . . . . 88

72 Labyrinth Seal design domain and boundary conditions with $\mathbf{u}$ based on Re number. . . . . . . . . . . . . . . . . . . 89

73 Geometry of a labyrinth seal using only diodicity. . . . . . . . . . . . . . 90

74 Modulus of plane velocity (flow from right to left) of geometry obtained with diodicity approach. . . . . . . . . . . . . . . . . . 91

75 Circumferential velocity plot of geometry obtained with diodicity approach. 91

76 Pressure field of geometry obtained with diodicity approach. . . . . . . . . 91

77 Objective function of diodes in a 2Dswirl system. . . . . . . . . . . . . . 92

78 Geometry with raised $W_{F}$ value. . . . . . . . . . . . . . . . . 92

79 Modulus of plane velocity of geometry with high $W_{F}$ value. . . . . . . . . . 92

80 Circumferential velocity plot of geometry with high $W_{F}$ value. . . . . . . . 93

81 Pressure field of geometry with high $W_{F}$ value. . . . . . . . . . . . . . 93

82 Restricted design domain of labyrinth seals. . . . . . . . . . . . . . . . . 93

83 Geometry of a labyrinth seal with a smaller design domain. . . . . . . . . . 93

84 Modulus of plane velocity of a labyrinth seal with smaller design domain. . 94

85 Circumferential velocity of a labyrinth seal with smaller design domain. . . 94

86 Pressure field of a labyrinth seal with smaller design domain. . . . . . . . . 94

87 Modified design domain with $r_{\min }=0.05 \ldots \ldots \ldots$. . . . . . . 95

88 Modulus of plane velocity of design domain with $r_{\text {min }}=0.05 \ldots$. . . . . 95

89 Circumferential velocity of design domain with $r_{\min }=0.05 \ldots \ldots . . . .95$

90 Pressure field of design domain with $r_{\text {min }}=0.05 . \ldots \ldots$. . . . . . 95

91 Modified design domain with $\operatorname{kmax}=10^{10} \ldots \ldots \ldots$. . . . . . . 96

92 Modulus of plane velocity with $\operatorname{kmax}=10^{10} \ldots \ldots \ldots$. . . . . . . 96

93 Circumferential velocity plot with $\operatorname{kmax}=10^{10} \ldots \ldots \ldots$. . . . . . 96 
94 Pressure field with $\operatorname{kmax}=10^{10} \ldots \ldots \ldots$. . . . . . . . 96

95 Modified design domain with $\operatorname{kmax}=10^{10}$ and $r_{\min }=0.20 \ldots \ldots 97$

96 Modulus of plane velocity of modified design domain with $\mathrm{kmax}=10^{10}$

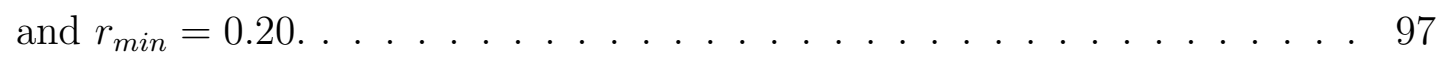

97 Circumferential velocity plot of modified design domain with kmax $=10^{10}$

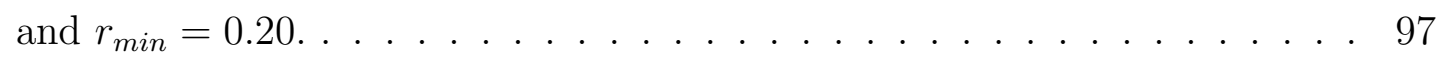

98 Pressure field of modified design domain with $\mathrm{kmax}=10^{10}$ and $r_{\min }=0.20 .98$

99 3D apperance of labyrinth seal considering diodicity. . . . . . . . . . . . 98

100 Vorticity with $\mathrm{kmax}=10^{10}$ and $r_{\min }=0.20 \ldots \ldots \ldots$. . . . . . 100

101 Modulus of plane velocity of modified design domain with vorticity. . . . . 100

102 Circumferential velocity plot of modified design domain with vorticity. . . . 100

103 Pressure field of modified design domain with vorticity. . . . . . . . . . 100

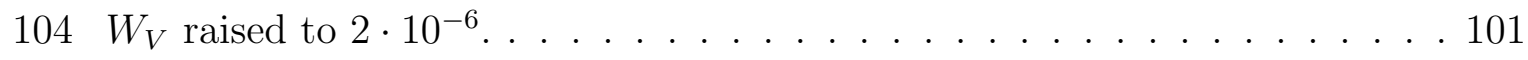

105 Modulus of plane velocity with $W_{V}=2 \cdot 10^{-6} \ldots \ldots \ldots 10$. . . . . . 101

106 Circumferential velocity plot with $W_{V}=2 \cdot 10^{-6}$. . . . . . . . . . . 101

107 Pressure field with $W_{V}=2 \cdot 10^{-6} \ldots \ldots \ldots \ldots 1$

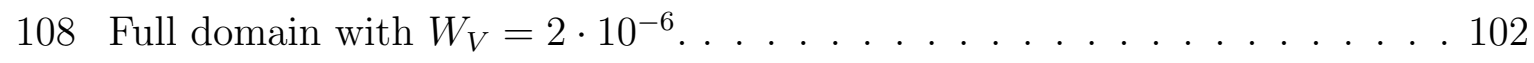

109 Modulus of plane velocity of full domain with $W_{V}=2 \cdot 10^{-6}$. . . . . . . 102

110 Circumferential velocity of full domain with $W_{V}=2 \cdot 10^{-6} \ldots \ldots$. . . . 102

111 Pressure field of full domain with $W_{V}=2 \cdot 10^{-6} \ldots \ldots \ldots$. . . . . . . 103

112 3D apperance of labyrinth seal considering vorticity. . . . . . . . . . . . . 104

113 Baseline for labyrinth seals comparison, respecting the same volume constraint. . . . . . . . . . . . . . . . . . . . 105

114 Boundary conditions of a rotating labyrinth seal. . . . . . . . . . . . 105

115 Labyrinth seal using fluid structure interaction algorithm. . . . . . . . . 106

116 Modulus of plane velocity in the Labyrinth seal using fluid structure interaction algorithm. . . . . . . . . . . . . . . . . 107 
117 Circumferential velocity plot in the Labyrinth seal using fluid structure interaction algorithm. . . . . . . . . . . . . . . . . 107

118 Pressure field in the Labyrinth seal using fluid structure interaction algorithm.107

119 Objective Function in a full optimization problem considering FSI. . . . . . 108

120 Displacement in red due to flow in Labyrinth seal. . . . . . . . . . . . . . . 108

121 3D apperance of labyrinth seal of a full optimization problem. . . . . . . . 109

122 Aspect ratio influence . . . . . . . . . . . . . . . . . . . . 109

123 Aspect ratio influence . . . . . . . . . . . . . . . . . . . . 110

124 Modulus of plane velocity of unit cell for different aspect ratio. . . . . . . . 110

125 Circumferential velocity plot of unit cell for different aspect ratio. . . . . . 110

126 Pressure field of unit cell for different aspect ratio. . . . . . . . . . . . . . 111

127 Deformation of geometries from different aspect ratio influence . . . . . . . 111

128 Topology optimization results for different shaft diameters. . . . . . . . . . 112

129 Modulus of plane velocity for different shaft diameters. . . . . . . . . . . 113

130 Circumferential velocity plot for different shaft diameters. . . . . . . . . . 114

131 Pressure field for different shaft diameters. . . . . . . . . . . . . . 115

132 Deformation of topology results for different shaft diameters. . . . . . . . 116

133 Topology optimization results for different rotational values. . . . . . . . 117

134 Velocity field of topology results for different rotational values. . . . . . . . 118

135 Circumferential velocity field of topology results for different rotational values. . . . . . . . . . . . . . . . . . . . . . . . . . . . 119

136 Pressure field of topology results for different rotational values. . . . . . . . 120

137 Deformation of topology results for different rotational values. . . . . . . . 121

138 Labyrinth seal with null circumferential velocity. . . . . . . . . . . . . . . . 121

139 Modulus of plane velocity in the Labyrinth seal with null circumferential velocity. . . . . . . . . . . . . . . . . . . . 122

140 Pressure field in the Labyrinth seal with null circumferential velocity. . . . 122 
141 Displacement in red due to flow in Labyrinth seal with null circumferential velocity. . . . . . . . . . . . . . . . . . 122

142 Topology results for different volume constraints. . . . . . . . . . . . . . 123

143 Modulus of plane velocity for different topology results with different volume constraints. . . . . . . . . . . . . . . . . . . . . . . . . 124

144 Modulus of plane velocity for different topology results with different volume constraints. . . . . . . . . . . . . . . . . . . . . . . . . 124

145 Modulus of plane velocity for different topology results with different volume constraints. . . . . . . . . . . . . . . . . . . . 125

146 Deformation of topology results for different volume constraints. . . . . . . 126

147 Comparison in velocity plot. . . . . . . . . . . . . . . . . . 141

148 Velocity magnitude over line. . . . . . . . . . . . . . . . . . . 141

149 Comparison in pressure plot. . . . . . . . . . . . . . . . . . . . . 142

150 Difference of calculated sensitivity and finite difference . . . . . . . . . . 143

151 Identification of cell in the calculated sensitivity field. . . . . . . . . . . . . 144 


\section{LIST OF TABLES}

2 Functional value $G$ comparison [CHALLIS; GUEST, 2009] for resulting topologies of double channel problem (mesh: 135000 elements $(\delta=1.5)$ and 14400 elements with $\delta=1.0 m$ and $\left.\kappa_{\max }=2.5 \cdot 10^{6}\right) \ldots \ldots$. . . . 70

3 Mesh size used in mesh independence test . . . . . . . . . . . . . 71

4 Parameters used in the fluid diode optimization . . . . . . . . . . 85

5 Diodicity Values for optimized geometries. . . . . . . . . . . . . . 89

6 Optimization parameters in the $2 \mathrm{D}$ swirl model . . . . . . . . . . . . . 90

7 Diodicity values in $2 \mathrm{D}$ swirl system. . . . . . . . . . . . . . . . 99

8 Optimization parameters considering vorticity . . . . . . . . . . . . . 99

9 Performance of optimized labyrinth seal including vorticity terms (units:SI). 103

10 Optimization parameters in a FSI problem . . . . . . . . . . . 106

11 Performance of optimized labyrinth seal using FSI (units:SI) . . . . . . . 108

12 Performance of optimized labyrinth seal using FSI with different aspect ratio considering only one cell. . . . . . . . . . . . . . . . . . . . 111

13 Performance of optimized labyrinth seal using FSI with different shaft diameters.

14 Performance of optimized labyrinth seal using FSI for different rotational values.

15 Performance of optimized labyrinth seal using FSI for different rotational values.

16 Performance of optimized labyrinth seal using FSI for different volume constraints.

17 Parameters used to CFD simulation . . . . . . . . . . . . . . . 140

18 Optimization parameters used to check gradient . . . . . . . . . . . 143

19 Parameters used in the fluid diode optimization . . . . . . . . . . . . . 144 


\section{NOTATION}

$\begin{array}{ll}\mathbf{u} & \text { Velocity } \\ \mathbf{v} & \text { Test function similar to velocity } \\ p & \text { Pressure field } \\ q & \text { Test function similar to pressure } \\ \mu & \text { Dynamic viscosity } \\ \rho & \text { Fluid density } \\ t & \text { Time } \\ \mathbf{f} & \text { External forces } \\ \mathbf{s} & \text { Position vector } \\ \mathbf{r} & \text { Horizontal component of the coordinate system } \\ \theta & \text { Angular component of the coordinate system } \\ \mathbf{z} & \text { Vertical component of the coordinate system } \\ \mathbf{e}_{\mathbf{r}} & \text { Unit vector of r } \\ \mathbf{e}_{\theta} & \text { Unit vector of } \theta \\ \mathbf{e}_{\mathbf{z}} & \text { Unit vector of z } \\ \mathbf{u}_{a b s} & \text { Absolute velocity } \\ \mathbf{u}_{r} & \text { Relative velocity } \\ \eta & \text { Horizontal component of finite element } \\ \epsilon & \text { Vertical component of finite element } \\ \omega & \text { Circumferential velocity } \\ \Omega & \text { Desing domain } \\ V & \text { Vector function space } \\ \hat{V} & \text { Test vector function space } \\ \Phi & \text { Energy dissipation } \\ \kappa & \text { Absortion coefficient } \\ \alpha & \text { Inverse permeability } \\ \boldsymbol{\sigma} & \text { Mechanical stress } \\ \boldsymbol{\epsilon} & \text { Strain of structure } \\ J & \text { Objective function } \\ L & \text { Sensitivity of an objective function } \\ \mathbf{F} & \text { Forward problem } \\ & \end{array}$




\section{CONTENTS}

$\begin{array}{llr}1 & \text { Introduction } & 18\end{array}$

1.1 Fluid Diodes . . . . . . . . . . . . . . . . . . . . . . . . 18

1.1.1 Labyrinth Seals . . . . . . . . . . . . . . . . . 20

1.1.1.1 Modeling and optimization of labyrinth seals . . . . . . . 25

1.1.1.2 The importance of the rotational speed . . . . . . . . . 29

1.2 Topology Optimization Method $(\mathrm{TO}) \ldots \ldots . \ldots . \ldots . \ldots$

1.2.1 Bibliography review of topology optimization . . . . . . . . . 31

1.2.2 Topology optimization for fluids . . . . . . . . . . . . . . . . . 34

1.3 Motivation . . . . . . . . . . . . . . . . . 35

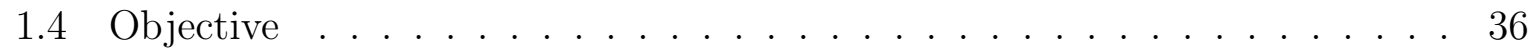

1.5 Scientific Contributions . . . . . . . . . . . . . . . 36

2 Fluid flow modeling $\quad 38$

2.1 Hypothesis . . . . . . . . . . . . . . . . . . . 38

2.2 The Navier-Stokes Equations . . . . . . . . . . . . . . . . . . . . . . 39

2.2.1 Strong Formulation . . . . . . . . . . . . . . . . . 39

2.2.2 Weak Formulation in 2D plane model . . . . . . . . . . . . . . 40

2.2.3 Weak formulation in 2D Swirl Model . . . . . . . . . . . . . . . 41

2.3 Finite Element Method for fluid Modelling . . . . . . . . . . . . . . . . . . 42

3 Topology Optimization Method 45

3.1 Material Model for Topology Optimization . . . . . . . . . . . . . . . 45

3.2 Objective Functions . . . . . . . . . . . . . . . . . 46

3.2.1 Total Pressure Loss . . . . . . . . . . . . . . . . . . . 47 
3.2.2 Energy Dissipation . . . . . . . . . . . . . . . . . . 47

3.2.3 Diodicity . . . . . . . . . . . . . . . . . . . . 48

3.2 .4 Vorticity . . . . . . . . . . . . . . . . 50

3.2.5 Fluid-Structure interaction (FSI) . . . . . . . . . . . . 51

3.2.6 Filtering in topology optimization . . . . . . . . . . . . . . 52

3.3 The TOBS Method . . . . . . . . . . . . . . . . . 53

3.4 Movable rotation boundary condition $(\mathrm{MRBC}) \ldots \ldots \ldots \ldots$

3.5 Sensitivity Analysis . . . . . . . . . . . . . . . . . . . 58

4 Numerical Implementation $\quad 62$

4.1 Programming environment . . . . . . . . . . . . . . . . . 62

4.2 Optimization procedure . . . . . . . . . . . . . . . 64

$\begin{array}{llr}5 & \text { Results } & 66\end{array}$

5.1 Fluid topology optimization with TOBS . . . . . . . . . . . 66

5.1.1 Classical Stokes problem . . . . . . . . . . . . . . . 66

5.1.1.1 Mesh independence . . . . . . . . . . . . . . 70

5.1.1.2 Influence of $\kappa_{\max }$ parameter . . . . . . . . . . 72

5.1.1.3 Influence of the penalization term $(q) \ldots \ldots . \ldots 74$

5.1.1.4 Influence of $\varepsilon$ parameter . . . . . . . . . . . . . 75

5.1.1.5 Influence of $\beta$ parameter . . . . . . . . . . . . . 77

5.1 .2 Navier-Stokes problem . . . . . . . . . . . . . . . . 77

5.1.2.1 Low Reynolds number . . . . . . . . . . . . . . 78

5.1.2.2 Wide range of Reynolds numbers . . . . . . . . . . . . 80

5.1 .3 3D fluid problem . . . . . . . . . . . . . . . . 81

5.2 Fluid diodes . . . . . . . . . . . . . . . . . . . . 84

5.2.1 2D swirl fluid diodes design considering diodicity . . . . . . . . 89

5.2.2 2D swirl fluid diodes design considering vorticity . . . . . . . . . 99 
5.3 Complete optimization problem considering Fluid-Structure interaction in fluid diodes . . . . . . . . . . . . . . . . . . . . . . . . . 104

5.3.1 Aspect ratio influence . . . . . . . . . . . . . . . . . 109

5.3 .2 Diameter influence . . . . . . . . . . . . . . . . 111

5.3.3 Rotational speed influence . . . . . . . . . . . . . . . . 116

5.3.4 Volume Constraint influence . . . . . . . . . . . . . . . . . . . . . 122

6 Conclusions

6.1 Future work . . . . . . . . . . . . . . . . . . . . . . 129

References

Appendix A - Filter of the design variables

Appendix B - Fluid flow verification 


\section{INTRODUCTION}

\section{$1.1 \quad$ Fluid Diodes}

Fluid diodes are devices that control fluid flow direction. The application varies, however, one relevant case study is when it is desirable to keep fluid in one chamber with open channel to exterior environment.

Fluid diodes can be divided in three categories, the first contains a small sphere able to block fluid flow (see Figures 1a-1b), the second category has active parts able open and close the passage (see Figures 1c-1d). However, an interesting third category are passive devices which uses only the fluid flow to inhibit flow direction. The most common geometry of the third kind is the famous Tesla Valve where secondary flanges can create a fluid recirculation (see Figures 1e-1f). It was invented and patented (U.S. Patent 1329559) by Nikola Tesla in 1916. The resistance can reach 10 to 200 times greater in one direction compared to the other. 


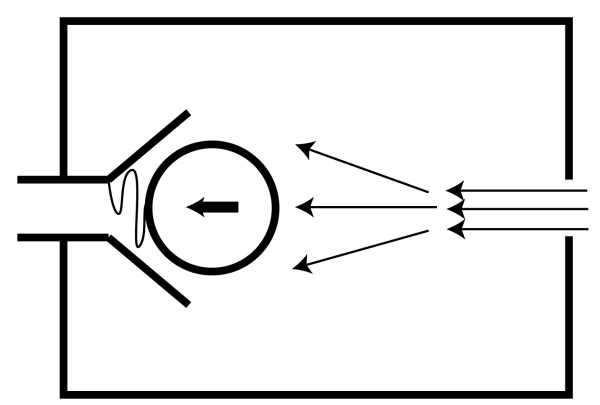

(a) Sphere blocking the flow from right to left.

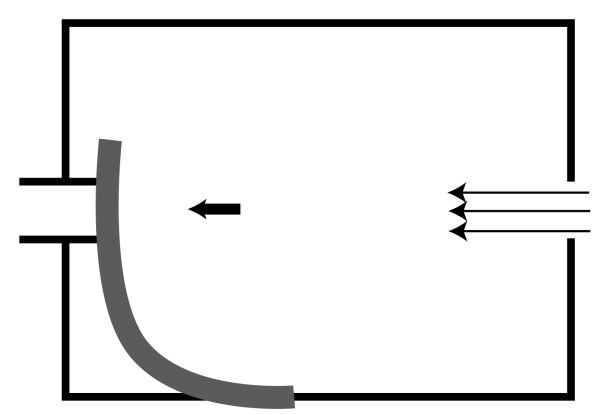

(c) Flexible active structure blocking the flow from right to left.

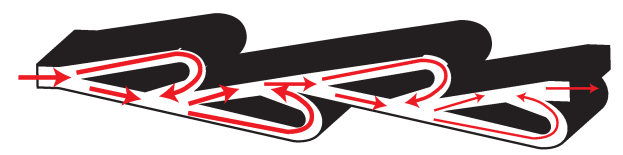

(e) Classic Tesla Valve with reduced flow from left to right

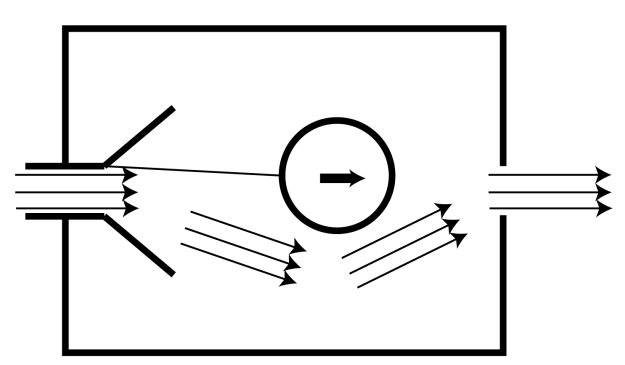

(b) Free flow from left to right

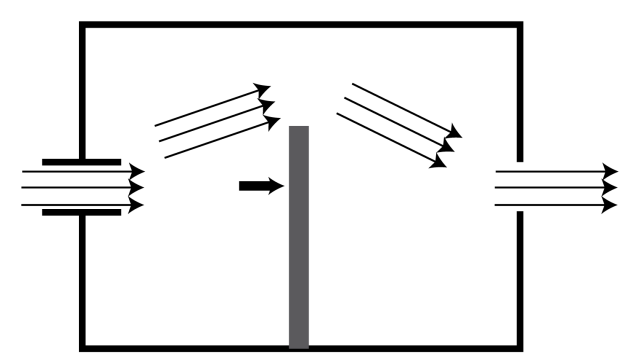

(d) Free flow from left to right

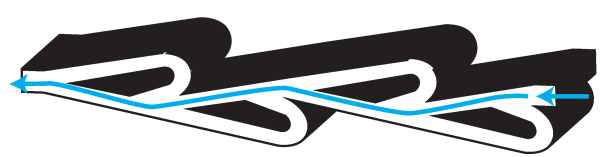

(f) Classic Tesla Valve with free flow from right to left

Figure 1: Possible fluid diodes devices.

Modern applications of fluid diodes are used in gas turbines (FDX Diodeß), compressors and pulsating heat pipes (PHP). A PHP is illustrated in Figure $2 \mathrm{a}$ in which it can be seen that there is a mixture of liquid and gas that is obligated to flow through small pipes due to a phase transformation occurred by heat exchange. Figure $2 \mathrm{~b}$ shows the use of fluid diodes inside the heat exchangers of a schematic PHP, stabilizing the pulsating flow and increasing the efficiency of the device.

The PHP are proven to be very effective in heat exchange [KHANDEKAR; DOLLINGER; GROLL, 2003; CHIEN et al., 2012; KHANDEKAR; GROLL, 2004] and the use of fluid diodes can considerably enhance its performance [VRIES et al., 2017]. 


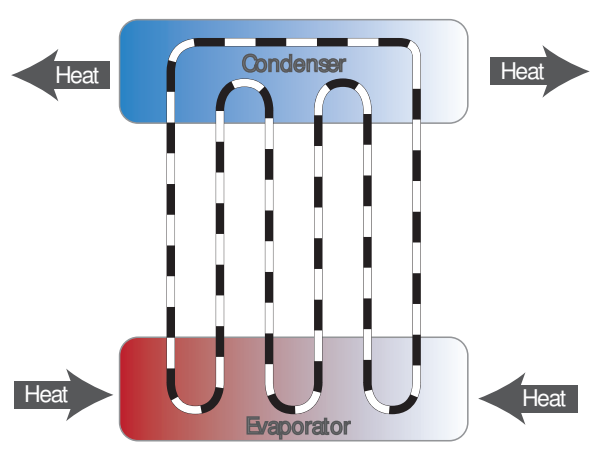

(a) Traditional pulsating heating pipe

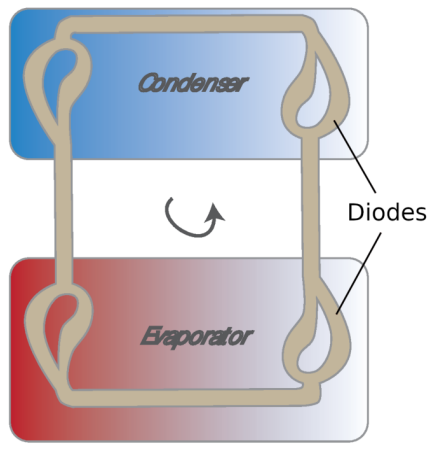

(b) Fluid diodes applied to pulsating heating pipe

Figure 2: Pulsating heating pipes.

\subsubsection{Labyrinth Seals}

There is a special type of fluid diode called Labyrinth Seals (LS) (Figure 3a) in which the reverse flow plays a more important role. LS are mechanical devices which inhibit the leakage from a chamber to another without closing the passage, i.e. keeping a gap between parts. In these devices a shaft is rotating and may not touch the stator due to wear conditions, however, fluid leakage must be avoided.

The application of LS varies a lot, however, it has been often used in turbines and compressors, mainly because the shaft of these devices reach high temperatures and speeds and this could cause wearing between rotating and fixed parts.

Figure 3b illustrates a labyrinth seal operation. Two different chambers with different pressures imply a flow leakage.

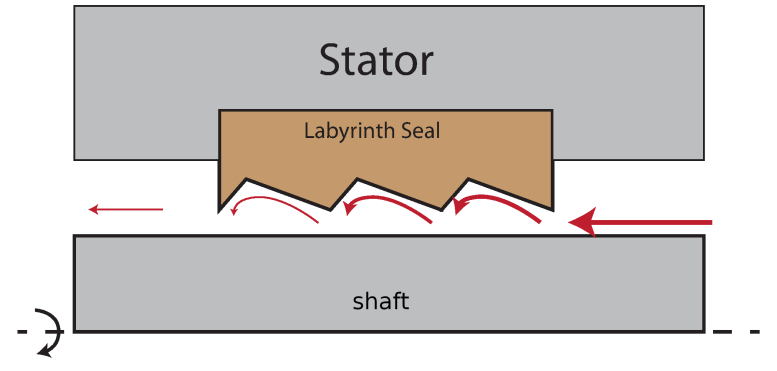

(a) Schematic labyrinth seal

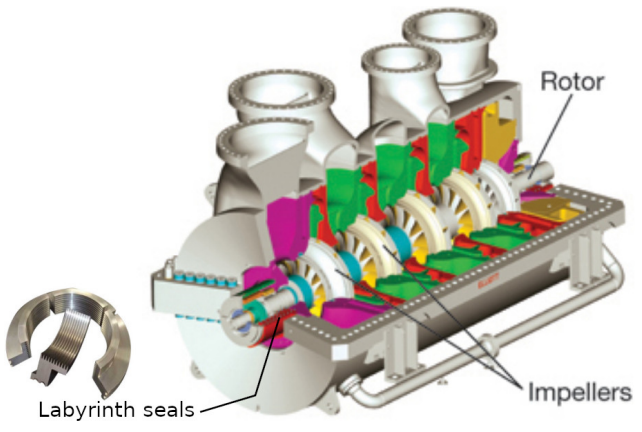

(b) Application in compressor

Figure 3: Labyrinth Seals in rotating environment.

A representation of a LS mounted in a turbine shaft is illustrated in Figure 4 and it is important to point out that these seals have been in use for decades in a variety of turbo-machinery applications [STOCKER, 1975, 1978; WYLER, 1981; MORRISON; 
CHI, 1985]. Labyrinth seals have numerous intrinsic other benefits including low maintenance, negligible running torque, simplicity, and reduced particulate contamination. However, typical labyrinth seals have an innate tendency to leak because there is no mechanical seal between the two areas of different pressures. Their leakage losses strongly affect the overall efficiency of the equipments.

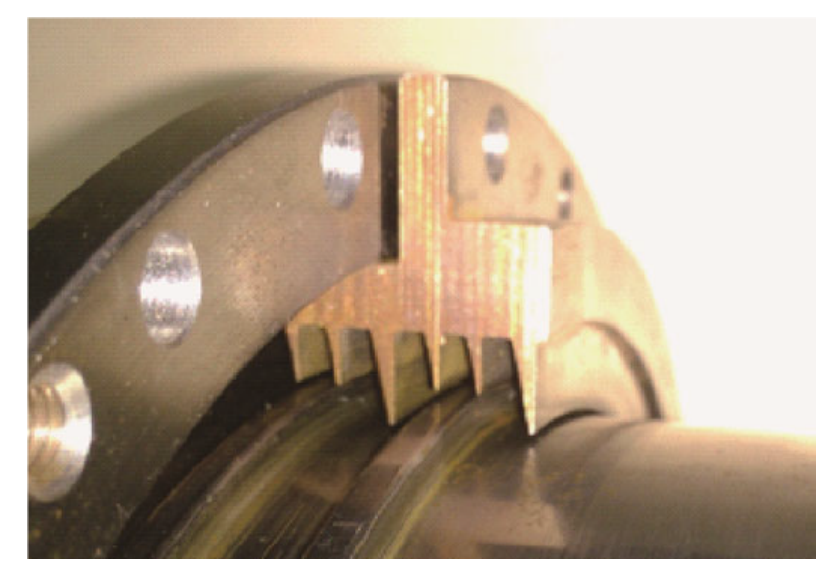

Figure 4: Actual Application of a Labyrinth Seal [LIN et al., 2014].

Labyrinth seals are often seen as suitable for both static and dynamic applications, having the advantages of being simple, reliable and tolerant to dirty and thermal expansion with minimal effects on the rotor dynamics [BOYCE, 2011]. An attractive feature of labyrinth seals is that they do not contact the rotating shaft. This has a direct impact on the integrity of the rotor and drastically decreases the cost of regular replacement and maintenance compared to other contacting seals, such as brush seals.

Although other types of contacting seals have been devised that provide better leakage characteristics, the reliance on contact between a rotating and non-rotating surface with these devices leads to unacceptable levels of wear. Other seal types include the viscosity [HEIDRICH, 1996] (for relatively high viscous fluids) and the brush seal [HENDRICKS et al., 1994] (limited by material properties). 


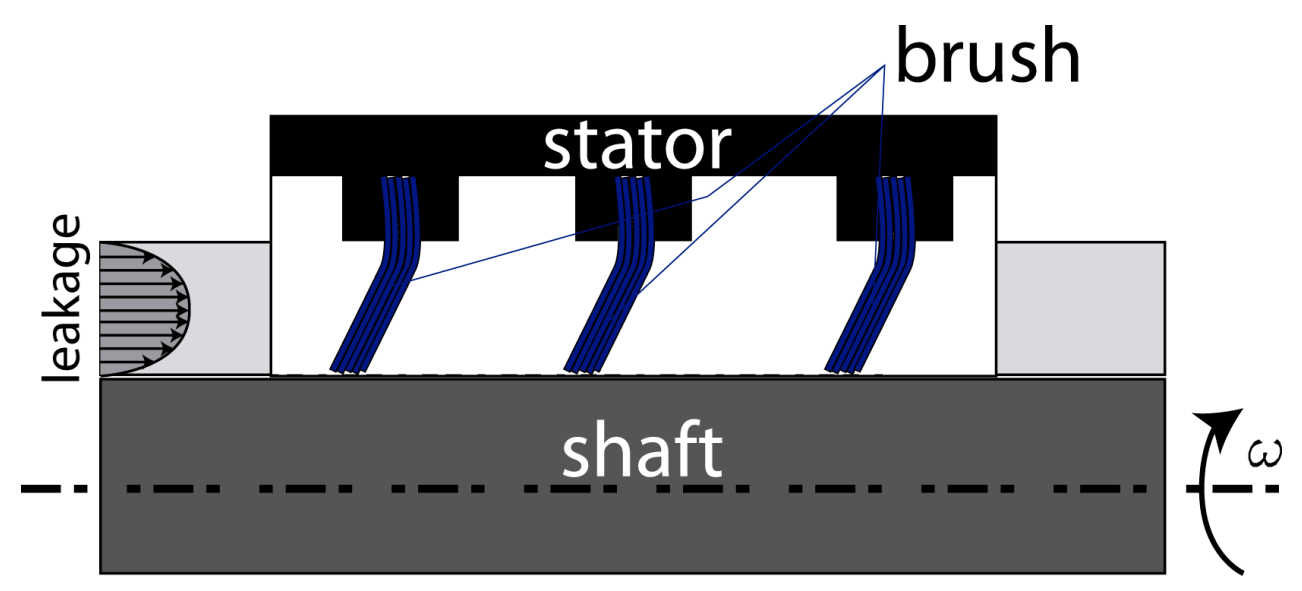

Figure 5: Brush seals

Recently, brush seals (Figure 5) have received special attention because they can be quite effective in reducing steam leakage. However, these seals do have certain shortcomings. Brush seals work well in reducing leakage if their clearance relative to the shaft is small. They are most effective as contact seals, for which life and wear rate are major concerns. At certain locations along the shaft, the lateral displacement of the shaft at critical speed during start up exceeds the brush seal limit, and the seal and shaft wear is quite high. For such situations, if the initial clearances for the seals are too small, the seal and the shaft may be damaged. The seal clearances after such a rub event are large, which reduces the seal performance. In addition, bristle loss and debris could lead to maintenance problems [HENDRICKS et al., 1994].

Advantages derived from the lowered maintenance and replacement costs in the end have led to renewed efforts in enhancing the performance of the labyrinth seal. The key design factor restricting leakage is the large total pressure drop produced by flow passing through multiple labyrinth seals. If not damaged, multiple labyrinth seals are capable of dropping the leakage stream pressure to a level near that of the exhaust pressure, thus reducing the leakage flow. The leakage flow through a labyrinth seal can be considered as a flow through a series of orifice restrictions. Figure 9 depicts a schematic representation of a labyrinth seal with vertical knives and steps, including the definition of key parameters. In a global sense, losses caused by individual restrictions and obstacles combine to produce a net energy loss to the system. The fluid, driven by the total pressure differential between $\mathrm{Pi}$ and $\mathrm{Pi}+1$ (as shown in Figure 6) is forced through a narrow clearance (restriction). As the fluid passes through the restriction (acting as an orifice), it undergoes an increase in velocity and a corresponding decrease in pressure with increased turbulence due to the sharp knife tip. At some point after the orifice, the fluid adjusts to the pressure 
condition in the next chamber. During this process, some of the kinetic energy of the fluid is recovered as a pressure rise, and some losses are converted to heat. The remaining total pressure of the fluid provides the pressure difference that forces the fluid to enter the next stage of the seal. Ideally, the kinetic energy of the fluid resulting from the previous stage of throttling will be dissipated before the fluid enters the next stage [KEARTON; KEH, 1952; MORRISON et al., 1983]. In this manner, by the time the fluid has travelled through all of the stages of the seal, its total pressure difference is greatly reduced, leading to negligible leakage flow through the seal.

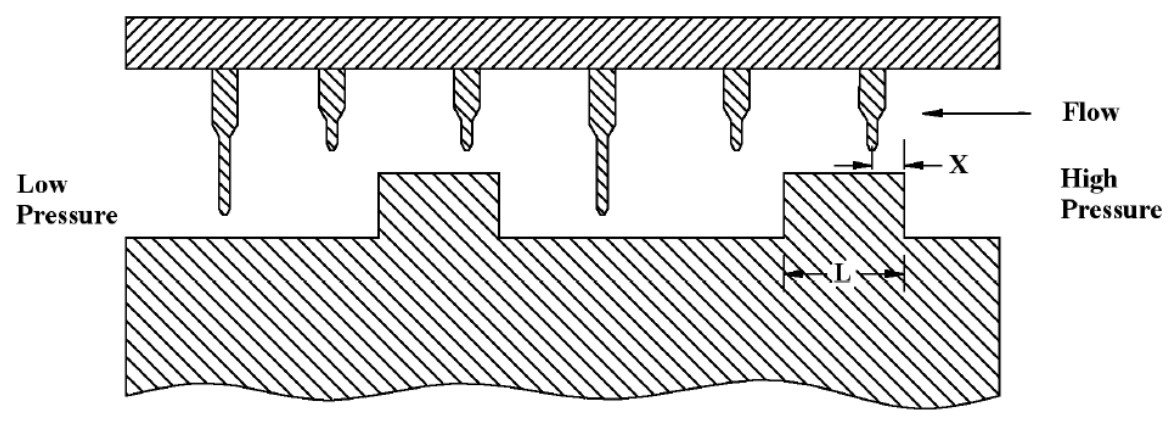

Figure 6: Schematic representation of a generic stepped labyrinth seal, depicting two cycles of a seal [VAKILI et al., 2006].

The application of labyrinth seals are wide, however, two main applications are very relevant: wet gas and natural gas. The former refers to the manufacturing of gasoline, i.e. when it occurs a wet gas stream, which is a gas mixture of saturated hydrocarbons, where additional condensing may happen in the suction pipes.

Natural Gas Seals are related to the gas transmission by pipeline, which requires a tremendous compressing capacity and it is the largest segment in the natural gas industry involving compressors and as a result labyrinth seals are very important to this field. Pneumatic devices release natural gas to the atmosphere as part of normal operation, however there are equipment that leak in excess of $4 \mathrm{~m}^{3}$ per day (high-bleed devices), releasing an average of 3,965 $\mathrm{m}^{3} /$ year per device [MCGETTIGAN, 2006]. Studies show that the major emission sources of methane are related to pneumatic devices/pumps and equipment leaks, accounting to approximately $60 \%$ of methane emissions [EPA, 2019]. As it can be seen in Figure 7, natural gas compression is also needed in the production of oil, because the so-called gas re-injection which requires the use of turbines and compressors.

When it comes specifically to compressors examples, seals are designed using labyrinth seals to prevent or restrict the process gas to leak to atmosphere or to mix the sealing liquid with the process gas stream. Mainly there are three classes of seals: mechanical (contact) face seal, liquid film seal and dry gas seal. 


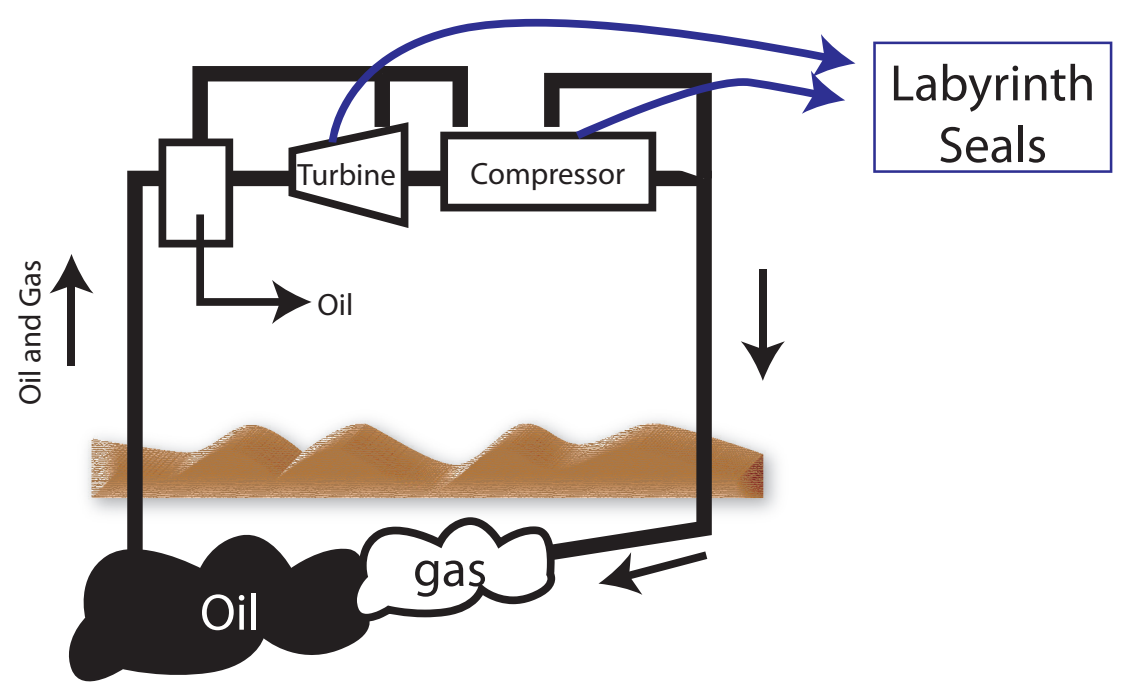

Figure 7: Natural Gas Reinjection.

Mechanical Face Seals (MFS) (Figure 8) uses liquid, such as oil, to lubricate, cool and create a sealing action to the sealing components during normal operation. The leak of mechanical face seal can be expected to be up to 10 gallons per day [ROSS; BECKINGER et al., 2003] of gas. The leakage of a MFS is considered dirty and contaminated, because most of the equipment with these seals drain the leakage to a separated area.

When the pressure is too high to use Mechanical Face Seal, another kind of seal called Liquid Film Seal (LFS) can be used. The principle of this seal is to maintain a film of oil between the gas side seals bushing and the shaft sleeve. Figure 9 shows a scheme of a liquid film seal.

A disadvantage of the LFS is the limitation on the use of hydrogen sulfide or chlorides as process gas, because there is usually corrosion in the system, increasing the clearance between the components. As a result, this can bring extremely high leakage rates for the sweet oil as well as contaminated oil [ROSS; BECKINGER et al., 2003].

Dry Gas Seal (DGS) is the most common kind of seal nowadays and it has been selling as a standard device, due to its reliability and environmental benefits. Figure 10 illustrates the principle of this seal, i.e. the gap between the rotating seal ring and the stationary seal ring is controlled by a spring, the process gas is usually used as a sealing medium and the labyrinth seal is always at the end shaft.

If the process gas is extremely hazardous, toxic or dirty, an external gas must be used as a sealing medium instead, for example nitrogenous. Besides that, if the process is dirty or can lead to phase changes, it must work with knockout vessels or mechanical separators 


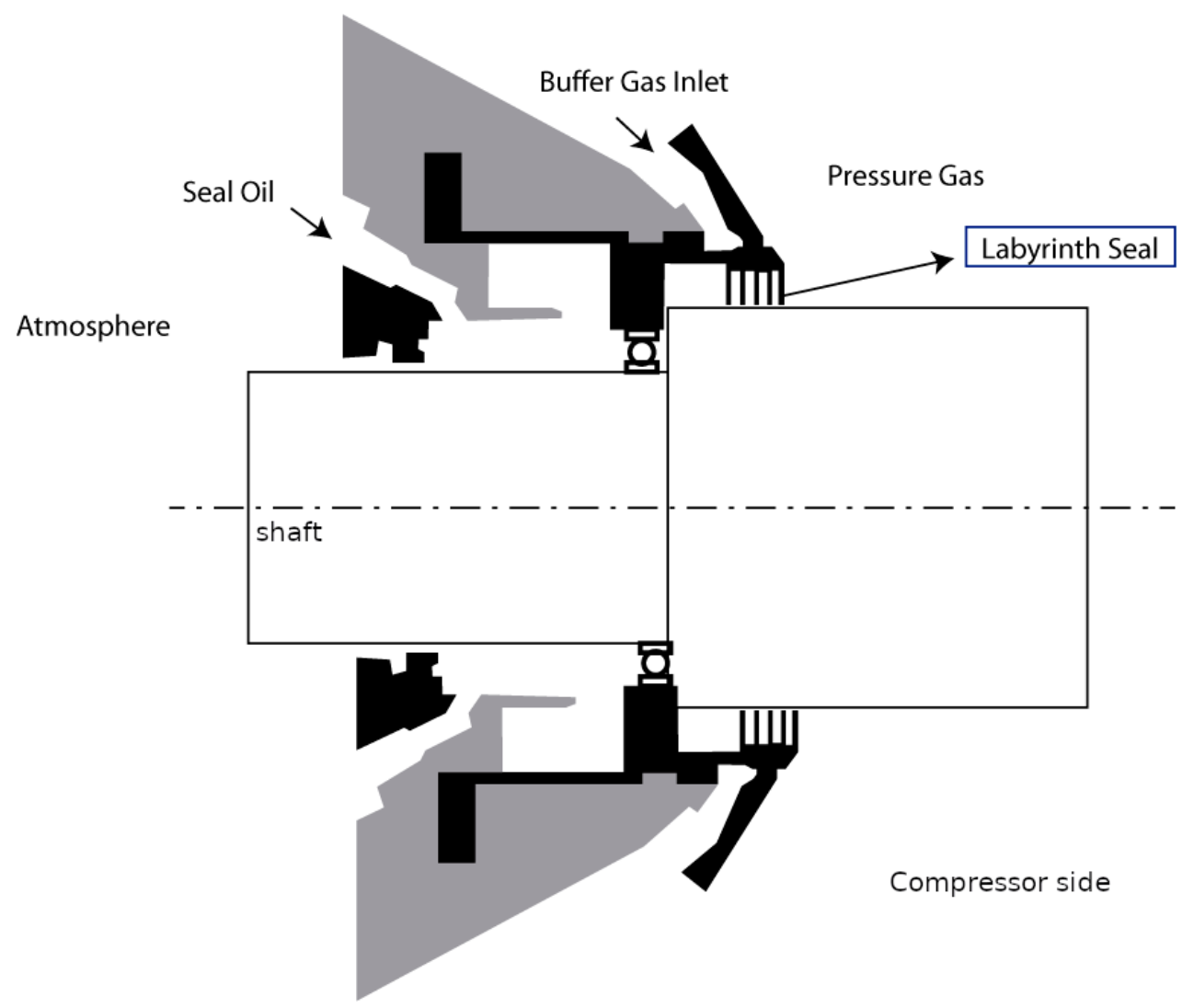

Figure 8: Mechanical Contact Face Seal (MFS).

in order to keep the dry gas seal operation.

\subsubsection{Modeling and optimization of labyrinth seals}

In a labyrinth seal, the flow locally changes direction often and rapidly speeds up and down as it negotiates a path through the seal. Total pressure is lost continuously through the seal, however there may be local rises in static pressure due to area changes, local stagnation points, and sudden expansions as the fluid flows into a chamber of the seal.

Methods for analyses of labyrinth seal leakage can be classified into two main categories: global models [KOMOTORI, 1977; TIPTON, 1986] and knife-to-knife models [MEYER; LOWRIE, 1974; ZHU; HE, 1990].

Global models approximate the labyrinth seal either by evaluating the cumulative effect due to the series of throttling losses or as a rough pipe model with uniformly distributed wall friction [NADA, a]. Knife-to-knife models (Figure 6) explicitly characterize the system geometry and calculate the internal flow through a seal by calculating relevant physical parameters as they change at various points internal to the seal [HESLER, 2005]. Although computationally intensive, this knife-to-knife method allows changing in flow 


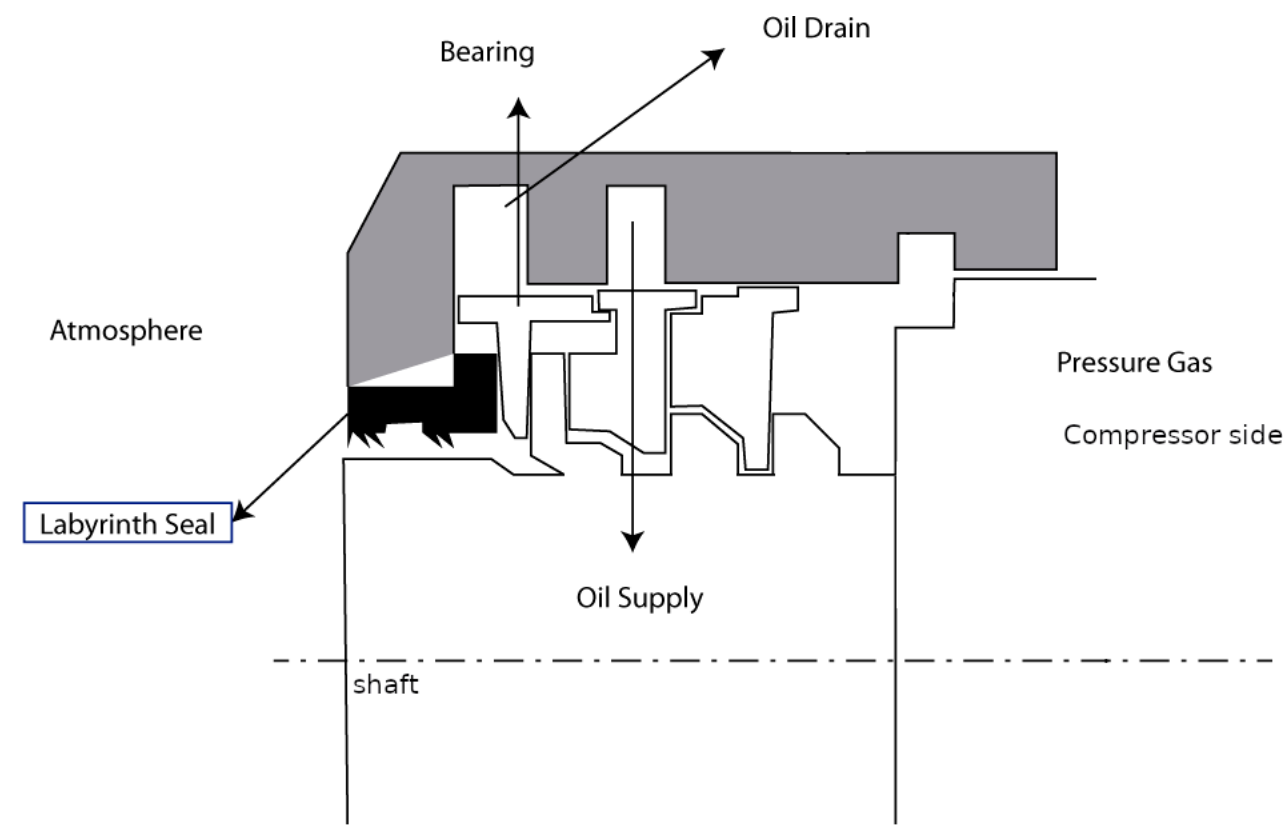

Figure 9: Liquid Film Seal (LFS).

behaviour at each throttling within the seal.

Although either of these approaches can yield good empirical correlations, the rough pipe model provides few information to the seal designer in terms of physically relevant design parameters such as knife spacing, shape, and sequence. A series of global models provide good results, however, encounter difficulty when calculating kinetic energy carryover. Additionally, problems are encountered predicting seal behavior in choked flow regimes [TIPTON, 1986].

Computational Fluid Dynamics (CFD) has been successfully applied to investigate selected labyrinth seal configurations [WITTIG et al., 1987; DEMKO; MORRISON; RHODE, 1989; LESCHZINER; DIMITRIADIS, 1989; RHODE; ADAMS, 2000; SCHRAMM et al., 2000; DENECKE et al., 2002]. The current available computer performance allows conducting CFD calculations for a high number of different seal geometries within a tolerable computing time. This allows the use of CFD for automated shape optimization of labyrinth seals, as it is frequently done to determine improved shaped airfoils and diffusers [CHOLASEUK; SRINIVASAN; MODI, 1999; SHAHPAR, 2000]. The effect of the labyrinth seal flow on rotor dynamics is also an important topic that has been studied extensively[CHILDS; SCHARRER, 1988; DEMKO; MORRISON; RHODE, 1990; VANCE; ZIERER; CONWAY, 1993; BASKHARONE; GHALI, 1994; PFAU et al., 2005].

It is important to mention some studies of fluid diodes focused specially in Labyrinth Seal, due to the wide application. First, there is a study [ABOULAICH et al., 2000] 


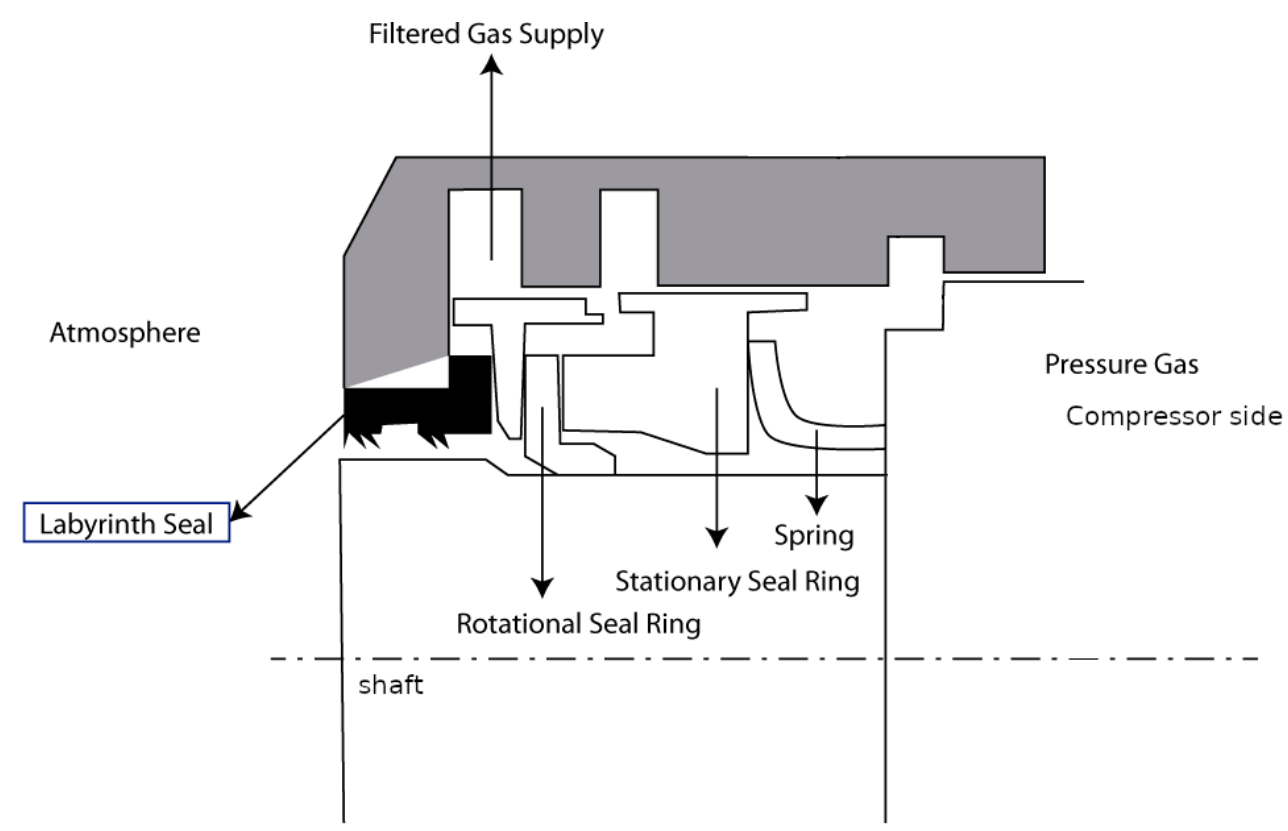

Figure 10: Dry Gas Seal (DGS).

which developed an improved shaped domain for a single cavity straight labyrinth seal, using a finite element code. They found an improved shape for incompressible, laminar flow conditions with minimal leakage.

Figures 11a and 11b show some possible seal geometry parameters that were improved by [SCHRAMM et al., 2004], considering for instance the step position SD/KP, the step height $\mathrm{SH} / \mathrm{C}$ and so on, obtaining reasonable values for pressure drop.

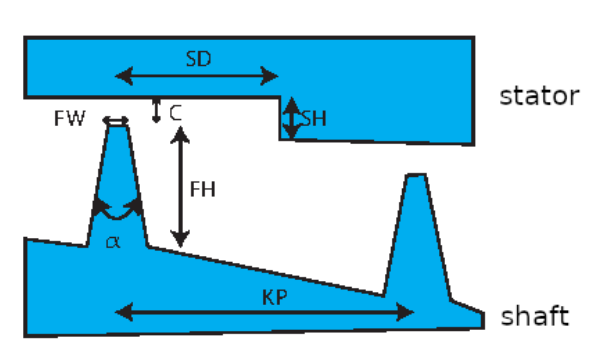

(a) Stepped labyrinth seal.

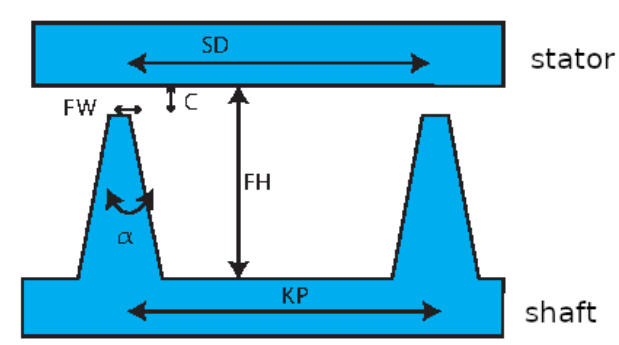

(b) Straight labyrinth seal.

Figure 11: Possible parameters for generic seals.

Considering for example Figure 11b, the flow passes the gap and hits the step. The jet is then strongly deflected and forms a stagnation point on the chamber ground below the step. Inside the labyrinth chamber, the jet separates into vortices. With increasing step distance $K P$, the stagnation point moves along the chamber ground to and then up the downstream fin. If the stagnation point reaches the region near the fin tip respective 
to the next gap, part of the kinetic energy of the jet is directly transferred to the next chamber. This mechanism is known as the carry-over effect and particularly occurs in straight-through labyrinth seals (Figure 11b), where it strongly impairs the sealing efficiency [HODKINSON, 1939; VERMES, 1961]. A numerical investigation on the influence of the teeth arrangement on the leakage fluid flow through the labyrinth seal were also been made [WANG et al., 2007].

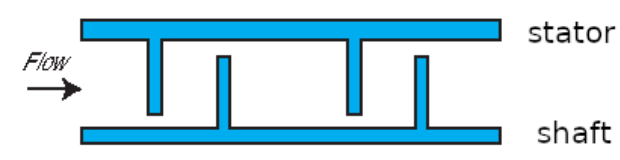

(a)

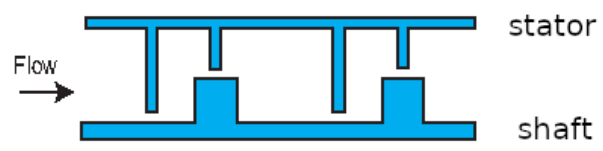

(b)

Figure 12: Possible LS geometries: (a)Interlocking (b)Traditional Stepped.

Figure 12 reveals a simple definition of two possible stepped labyrinth seal geometries: interlocking (Figure 12a) or traditional stepped seal and the traditional stepped seal (Figure 12b). One interesting result found by [WANG et al., 2007] is that the former reached a leakage larger than the latter, i.e the traditional stepped seal shows better performance. The numerical modeling of the flow through the labyrinth seals was performed by solving the Navier-Stokes equations and $\mathrm{k}-\epsilon$ turbulence model based on assumption of the axisymmetric flow.

[KIM; KANG, 2010] investigate the leakage characteristics of two labyrinth seals with different configurations: straight and stepped, in which the latter has been proved to be better than the former. In recent researches, [KIM; KANG; MOON, 2009; KIM; CHA, 2009] compare leakage characteristics between the straight and stepped seals, considering possible influences, such as clearance size between the standard configurations.

Labyrinth seals are widely employed in industry, with several layouts being studied [HESLER, 2005]. However, the optimization of these shapes is underexploited in literature, with works limited to parametric, shape optimization and genetic algorithms [BELLAOUAR; KOPEY; ABDELBAKI, 2013; SCHRAMM et al., 2004; WRÓBLEWSKI et al., 2010].

There are also studies [SCHRAMM et al., 2004], which applied the simulated annealing method to perform a shape optimization aiming at minimizing the leakage mass flow rate of a stepped labyrinth seal. Optimization applied to labyrinth seals have also been performed by [ASOK et al., 2007], however they have performed only parametric optimization in the labyrinth seals and unfortunately these methods restricts the final geometry to the initial guess and just few innovation can be achieved as illustrated in 
Figure 15.

[ABOULAICH et al., 2000; SCHRAMM et al., 2004] have performed shape optimization, and they highlight the importance of the geometry in a labyrinth seal, concerning its performance. Based on the same idea, [ASOK et al., 2007], have performed a Neural network and CFD-based optimization of square cavity and curved static labyrinth seals. The study performed by [ASOK et al., 2007] have the objective of maximizing the pressure drop of the system, which depends on a turbulent coefficient and the drag between the flow and the wall. The interesting conclusion of this study is that the intuitive analysis (see Figure 13) is much better than the neural optimization itself and that is possibly due the fact that the geometry plays a more important role than its parameter. Thus, it can be concluded and reinforced that the topology optimization is a promising method to this field.

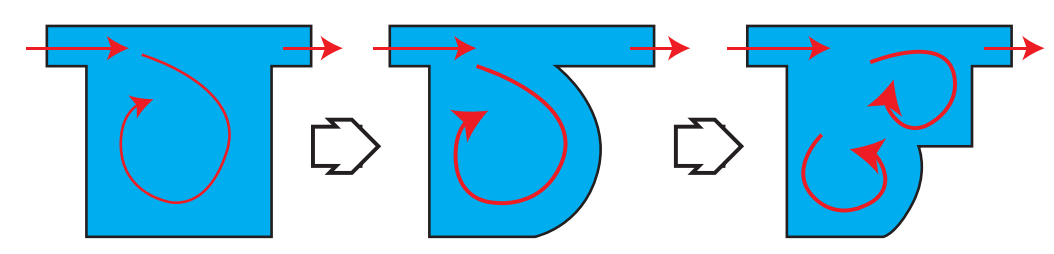

Figure 13: Optimization of labyrinth seal based on intuition.

\subsubsection{The importance of the rotational speed}

One important effect that is explored in this work is the influence of the rotation of the shaft in labyrinth seal design as illustrated in Figure 14. It was reported [WASCHKA et al., 1993] that the effect of shaft rotation is important only when the rotating speed is very high (more exactly, when the ratio between the circumferential speed of the seal arm and the flow speed is very large). There is also a study [SUBRAMANIAN; SEKHAR; PRASAD, 2015] about leakage characteristics, influenced by centrifugal and thermal radial growth computing a generic rotating labyrinth seal used in the gas turbine secondary air system. In this study, the combined influence of seal location and its radial growth on the leakage performance is also investigated. Three-dimensional Reynolds Averaged NavierStokes compressible equations are solved together with the additional transport equations for the turbulent kinetic energy and the turbulent dissipation rate. A standard $k-\epsilon$ model, with enhanced wall treatment, available in ANSYS FLUENT 14, has been incorporated into the analysis. The conclusion was that the leakage flow rate decreases considerably 
with centrifugal growth and further gets reduced drastically as the thermal growth is also included, regardless of location. The effect is more pronounced at high temperature and high pressure ratio, as the radial position of the seal is increased. Besides, the influence of rotational speed up to $2000 \mathrm{rad} / \mathrm{s}$ plays important role up to $22 \%$ [SUBRAMANIAN; SEKHAR; PRASAD, 2015].

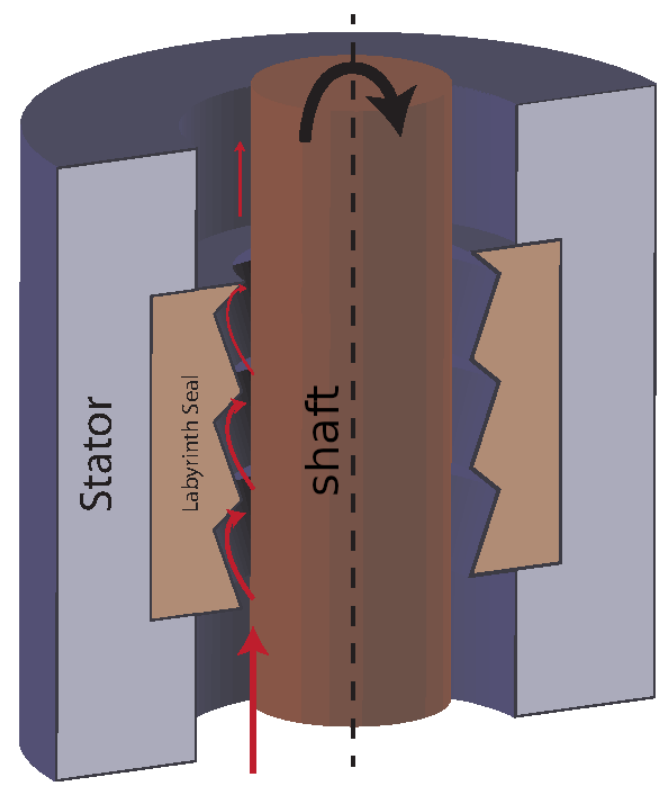

Figure 14: Illustration of the effect on the flow due to the rotation of an axis.

With the purpose of studying better the rotational speed with respect to the possible optimized geometries, this work also explores this parameter in optimization results.

\subsection{Topology Optimization Method (TO)}

Optimization is the search for the best possible solution to a problem with an objective function, design variables, and constraints. The optimization in Engineering currently has three forms: the parametric, shape and topology optimization as it can be seen in Figure 15. The first operates with dimensions or ratio of dimensions, however, there is no change in the shape of the structure. The second kind modifies the internal or external contours of the structure by means of polynomials or splines, however, there is no addition of new gaps in the part. Finally, topology optimization method, here referred to as TO, consists in distributing the material in the studied area to optimize (maximize or minimize) the objective function subjected to some constraints. This is accomplished by assigning a design variable which is usually a pseudo-density ranging from 0 to 1 , with 0 being the absence material 1 and the presence of material. 


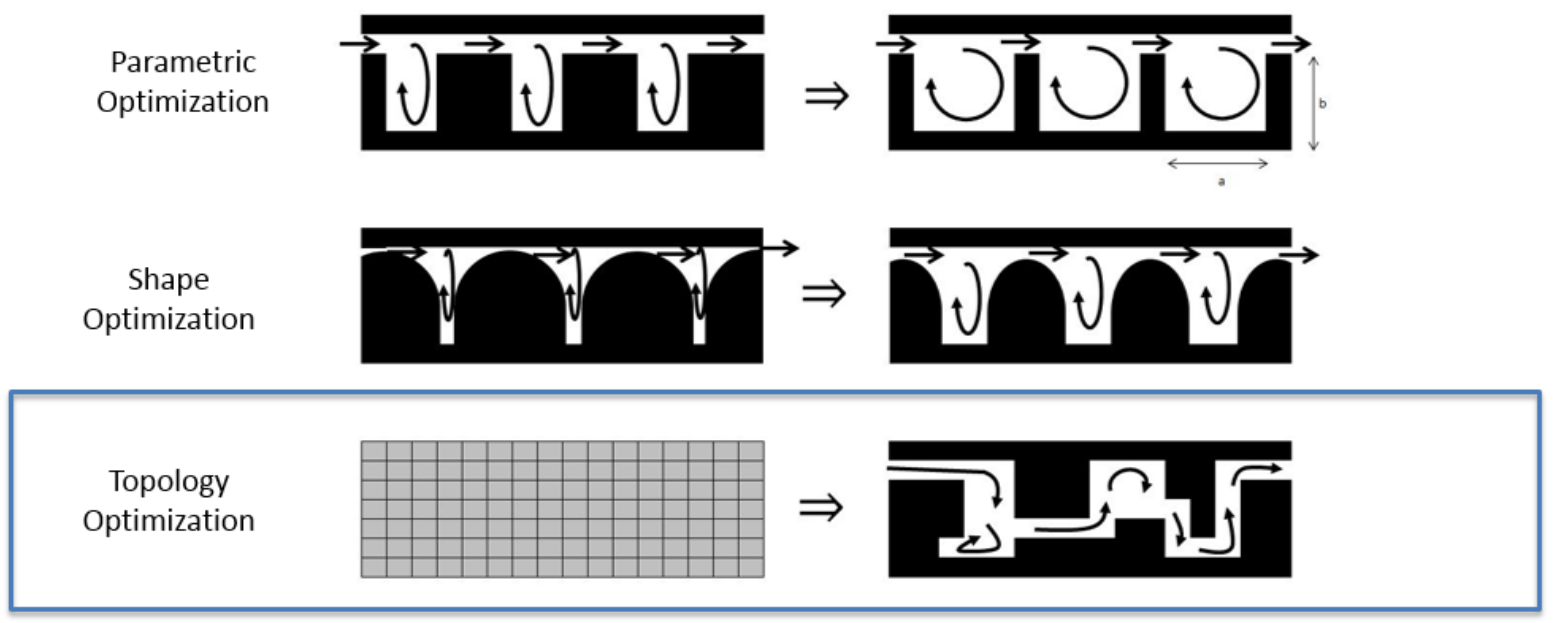

Figure 15: Possible types of fluid diodes optimization.

The topology optimization procedure can be seen in Figure 16, where the design domain can be discretized, optimized and the found solution can be verified and then manufactured.

With the previous studies of the literature in mind, it is possible to imagine the wide of possible solutions to the fluid diode design, specially the labyrinth seals.

\subsubsection{Bibliography review of topology optimization}

The first successful implementation of topology optimization was made by [BENDSØE; KIKUCHI, 1988] for structural design applications. In their works, the homogenization method [BENDSØE; KIKUCHI, 1988] and SIMP (Solid Isotropic Material with Penalization) method [ROZVANY; ZHOU; BIRKER, 1992; BENDSØE; SIGMUND, 1999] were developed. The former employs homogenization concept on micro-structure to represent lay out of the material and void region. The latter uses the relationship between material properties and element density to be optimized. In SIMP method, the final optimization problem can be stated as finding the optimized distribution of element densities in the structure.

SIMP method is easy to implement and efficient due to good harmony with Finite Element Method (FEM) and thus, it has been widely used in a broad range of Engineering design problems such as linear elastic, dynamics, fluid mechanics and multi-physics. The numerous works on the conventional topology optimization can be found in [BENDSOE; SIGMUND, 2013] and references therein. Topology Optimization method has its inherent numerical instabilities such as checkerboard patterns, mesh dependency and minimum member size control, although they can be avoided by several techniques and con- 


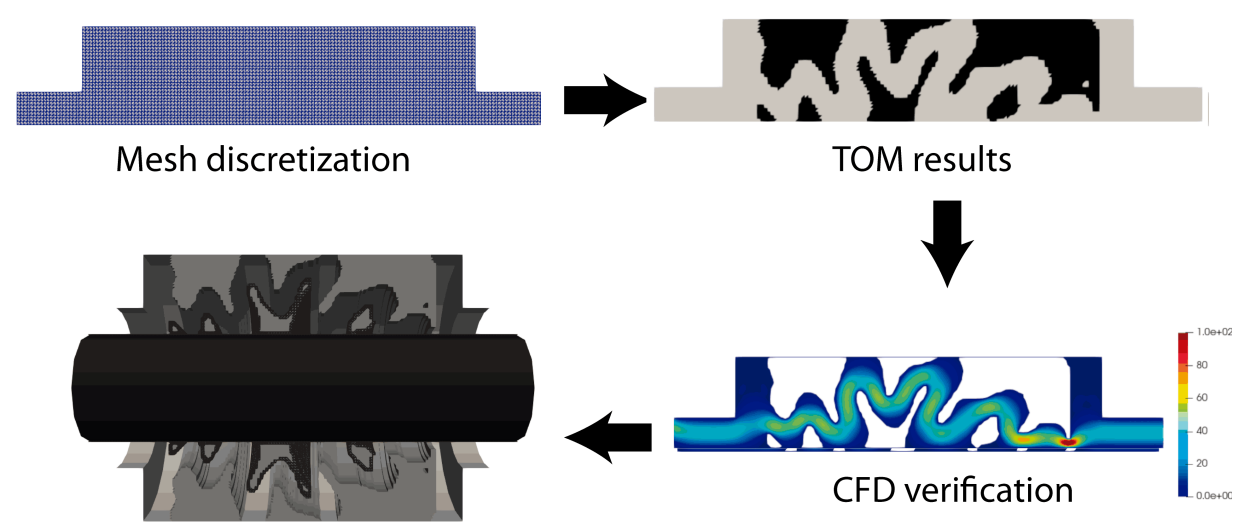

Prototype manufacturing

Figure 16: Topology optimization procedure for a labyrinth seal.

straints such as perimeter control [HABER; JOG; BENDSØE, 1996], density redistribution [YOUN; PARK, 1997], slope constraint [PETERSSON; SIGMUND, 1998; BOURDIN, 2001; ZHOU; SHYY; THOMAS, 2001] and density or sensitivity filtering [SIGMUND; PETERSSON, 1998; BRUNS; TORTORELLI, 2001; BOURDIN, 2001; GUO; GU, 2004].

Xie and Steven proposed the evolutionary structural optimization (ESO) based on element-wise stress level [XIE; STEVEN, 1993, 1994; QUERIN; STEVEN; XIE, 1998; KIM et al., 2002]. In the ESO, optimized topology of a structure can be represented by adding and/or subtracting elements in a heuristic way. [YOON; KIM, 2005] proposed a new topology optimization methodology named element connectivity parameterization (ECP). In the ECP, the elastic links are assumed to exist at vertices of elements and employed as design variables to determine existence of elements connected by them. The ECP can provide excellent designs in geometrically nonlinear problems since unrealistic effect on distortion of low density elements is eliminated. The material cloud (MC) method was proposed by [CHANG; YOUN, 2006]. In this method, the optimized topology is expressed by means of the material clouds and the size and/or position of them are design variables. With this method, the change of design space can be easily achieved and computational costs can be reduced by introducing the active finite elements.

Since cell-based representations of topology leads irregular and vague boundary layouts, other attempts for smoothing material boundary have been studied. In recent years, level set based topology optimization which was first proposed by [SETHIAN; WIEGMANN, 2000] has been extensively investigated. The moving front which express material boundary is adopted as design variables. The evolution of material boundary 
is governed by Hamilton-Jacobi equation employing the shape velocity computed from design sensitivity analysis. Several works on level set based shape and topology optimization can be found in [WANG; WANG; GUO, 2003; ALLAIRE; JOUVE; TOADER, 2004; ALLAIRE; JOUVE, 2005; AMSTUTZ; ANDRÄ, 2006; WANG; WANG; GUO, 2004].

Despite the profound investigations on level set based topology optimization, it has a shortcoming which is concerned with the incapability of creating new inner fronts during the optimization process. Due to the shortcoming, an initial level set generally includes a number of inner fronts so that the optimized topology of level set based topology optimization is highly dependent on the initial number and positions of inner fronts. In order to overcome the shortcoming, level set based topology optimization attempts for free creation of new inner fronts based on the topological derivative [BURGER; HACKL; RING, 2004; ALLAIRE; JOUVE, 2005; AMSTUTZ; ANDRÄ, 2006] and the strain energy density [PARK; YOUN, 2008] have been presented. [BELYTSCHKO; XIAO; PARIMI, 2003] proposed a nodal implicit function based topology optimization using level set representation and extended finite element method (XFEM). Level set method may be able to provide clear boundaries during topology optimization process, however, the usual implementation is generally complex and it is not an easy task to setup the optimization parameters.

There has been several works on spline based topology optimization. The earliest spline based topology optimization is the bubble method [ESCHENAUER; KOBELEV; SCHUMACHER, 1994]. In this method, bubbles are inserted based on the characteristic function and shape optimization of the outer boundaries and inner bubbles which are represented by splines are sequentially repeated for topology optimization, however, the conventional FEM with remeshing scheme is used for analysis in the method.

The Topology optimization of binary structures (TOBS) was introduced by [SIVAPURAM; PICELLI, 2018; SIVAPURAM; PICELLI; XIE, 2018] and have a reasonable potential in TO for fluids, since it can eliminate gray scale, which requires some effort on setting up parameters in density method. Besides, TOBS can keep clear boundaries between fluid and solid, which is essential for simulations that requires determination of wall distance. Previous works in the literature reported that solving TO with binary variables is time consuming [BECKERS, 1999; SVANBERG; WERNE, 2006], however, literature[NADA, b] shows that the TOBS branch-and-bound implementation is efficient. 


\subsubsection{Topology optimization for fluids}

The application of the topology optimization method to fluid problems was introduced by [BORRVALL; PETERSSON, 2003], proposing the optimization of the flow in a 2D channel in order to minimize energy dissipation. In this case, the flow was modelled by the Navier-Stokes equation for incompressible low Reynolds numbers and to avoid intermediate values, they penalized the design variables with a factor, very similar to the SIMP ("Solid Isotropic Material with Penalization") method. The main idea is to discretize a design domain in finite elements, finite volumes or similar methods in order to solve a problem of finding which regions should be solid $(\rho=0)$ or fluid $(\rho=1)$. This requires relaxing the problem and acceppting temporarily intermediate values to minimize an objective function, which is usually energy dissipation, subjected to some constraints. As the optimization proceeds, the intermediate values (gray elements) in the design domain should disappear and a clear solution of a geometry should be obtained. The process of implementing topology optimization for fluids can be illustrated in Figure 16.

[GERSBORG-HANSEN; SIGMUND; HABER, 2005; OLESEN; OKKELS; BRUUS, 2006] extended the work of [BORRVALL; PETERSSON, 2003] by studying a wider range of Reynolds numbers, considering fluid inertial effects, and nonlinearity, through application of the Navier-Stokes equation. These implementation allow the design of devices based on the variation of the velocity of the fluid in the channel, as directional flow selectors, in which, according to the velocity, the fluid follows a different direction through the channel. [EVGRAFOV, 2005] compares the model applied by [BORRVALL; PETERSSON, 2003] with the Brinkman model with an approach that considers fluid viscosity as one of the problem variables. Afterwards, it is studied the extension of TO to fluids with quasi-compressibility effect [EVGRAFOV, 2006] and the effects of the application of topology optimization to non-Newtonian fluids [PINGEN; MAUTE, 2010]. Topology optimization has been also studied for rotational fluid flow [ALONSO et al., 2018] and considering non-newtonian fluids [ALONSO; SAENZ; SILVA, 2020]. Topological derivatives applied to fluids has been explored by [SÁ et al., 2016]. [YOON, 2016] applied topology optimization for turbulent flows modeled by the Spalart-Allmaras model. More recently, [DILGEN et al., 2018] used the Spalart-Allmares and $k-\omega$ models, showing that optimized designs outperform those optimized under a frozen turbulence assumption and [SÁ et al., 2021] expanded the topology optimization model of turbulent flows using Spalart- Allmaras model in rotating fluid domains. 
It is not common to found also articles about topology optimization for Fluid Diodes, however, [LIN et al., 2015] has published an article which uses topology optimization method for designing Tesla valves, that is, for designing fixed-geometry fluid diodes that allow easy passage of fluid flowing in one direction while inhibiting flow in the reverse direction. Also, an exploration of Pareto frontier of this problem was studied [SATO et al., 2017] as well as a comparision of 2D and 3D analysis in this context was performed [GUO et al., 2020]. As for the topology optimization applied to Labyrinth Seals, nothing was found in the literature.

The Topology Optimization of Binary Structures method in fluid flow problems has not been largely explored, since most of analysis relies on density method. However, the TOBS method combining Navier-Stokes equation with Darcy's law not only can help eliminating gray scale issues, making it clear where the boundary is located, but also simplify setting some material model penalization parameters along optimization process, which are used to avoid gray scale in density method.

This work extends the progress made by [LIN et al., 2015] with the labyrinth seal approach by using TOBS considering rotational speed of the shaft, influence of structural parts and implementation of movable rotational boundary condition that can detect if the parts belongs to stator or shaft.

\subsection{Motivation}

Uncontrolled methane emissions can have a great influence on climate impacts. The emission occurs in a variety of ways, such as a second product from crude oil production, leakage from labyrinth seals on compressors and turbines, fugitive leakage on facilities with a high number of equipment components, direct emissions from gas burned in pilot flame on flares and also from pneumatic devices, which are designed to vent gas as part of operation. Studies show that the major emission sources are related to pneumatic devices/pumps and equipment leaks, accounting to approximately $60 \%$ of methane emissions [EPA, 2019]. Pneumatic devices release natural gas to the atmosphere as part of normal operation, however there are equipment that leak in excess of $4 m^{3}$ per day (high-bleed devices), releasing an average of 3,965 $\mathrm{m}^{3}$ /year per device [MCGETTIGAN, 2006].

The leakage rate is dependent on maintenance and design, so typically the solutions orbit between replacing the high-leaking devices with low-leaking devices with focus on improved labyrinth seals and maintenance aimed at reducing losses. However, the main- 
tenance has a limited effectiveness, since it involves generally an operational pressure reduction. Thus, the most effective approach is to redesign labyrinth seals for equipment in the group of turbines and compressors.

Considering all the topology optimization capabilities, it can perform this redesign by bringing non-intuitive geometries, avoiding a long cycle of design, simulation and redesign. Besides, the use of TOBS method can provide clear solid-fluid boundaries and avoids the need of continuation schemes to eliminate gray scale. It can, thus, bring innovation to this field, fighting against undesirable emissions, through a systematic methodology to design such seals.

\subsection{Objective}

The main objective of this work is the development of a systematic design optimization method, based on the Topology Optimization Method (TO), applied to the design of fluid diodes focused on labyrinth seals, in order to reduce fluid leakage, using integer variables (the Topology Optimization of Binary Structures [NADA, c]). The goals of the project can be divided in:

- Development of a methodology to design passive dissipative fluid diodes focusing on labyrinth seal devices using topology optimization method;

- Apply the TOBS method to fluid devices design;

- Development of methods for dealing with moving rotational boundaries conditions and floating islands elimination in the topology optimization process.

\subsection{Scientific Contributions}

There are recent studies about fluid diodes and topology optimization, however this work believes to add the following scientific contributions:

- Development of a TO methodology to design fluid diodes focused on labyrinth seals.

- Extension of the TOBS methods to fluids[SOUZA et al., 2021], applying it to fluid flow problems, not only helping to eliminate gray scale issues, making it clear where 
the boundary is located, but also simplifying setting some material model penalization parameters along optimization process, being a simpler method to deal with these problems;

- Development of a classification algorithm to recognize geometries during TO, helping the implementation of a movable rotational boundary conditions;

- Define an objective function based on maximization of vorticity;

- Development of an objective function with structural terms that can eliminate undesirable floating islands in the results. 


\section{FLUID FLOW MODELING}

\subsection{Hypothesis}

In this section some hypothesis are explored and discussed in order to properly implement the method. Fluid diodes have a variety of application with a wide range of parameters related to rotation, dimensions and leakage.

In the application of labyrinth seals related to compressors and turbines, it is common to use turbulence models due to high velocities values, however this work uses low Reynolds number since it is a pioneer work using topology optimization and several challenges are faced, such as how to deal with solid parts in the middle of domain (floating islands) and how to develop a method that does not close the passage (gap) between rotor and stator.

Permanent regime is also considered in this work in order to simplify the initial models, letting transient regime analysis as a future work that can be studied.

Incompressibility is adopted in this work for being a resonable option, since compressible flow is the branch of fluid mechanics that deals with flows having significant changes in fluid density and that is not the case of this work. In order to distinguish between compressible and incompressible flow, the Mach number (the ratio of the speed of the flow to the speed of sound) must be greater than about 0.3 (since the density change is greater than $5 \%$ in that case) before significant compressibility occurs as illustrated in Figure 17. So, although it might be counter-intuitive, in these cases the density can be considered negligible [FOX; MCDONALD, 1994].

This incompressibility hypothesis may not be the case for higher rotational speed devices with thicker shaft and high pressure differences than the values used in this work. 


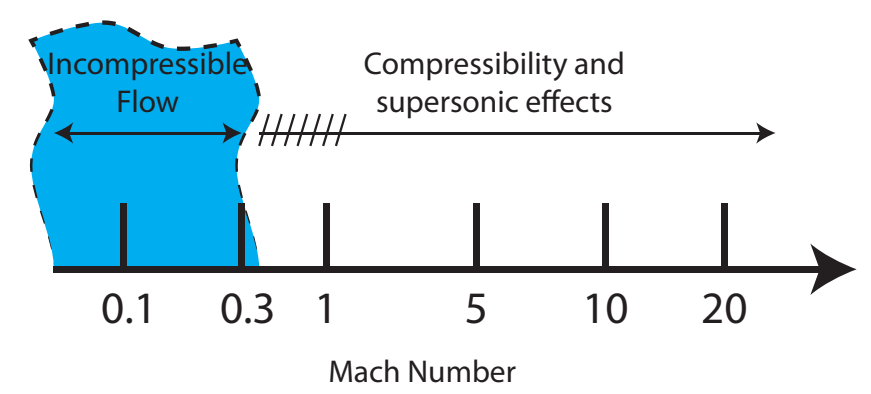

Figure 17: Incompressible flows according to Mach Number.

\subsection{The Navier-Stokes Equations}

In the next sections, it will be explored the most common equations for fluids, i.e. the Navier-Stokes equations, in order to apply them into the topology optimization method.

\subsubsection{Strong Formulation}

In order to simulate the fluid diodes, first it is necessary to write the complete NavierStokes equation considering all hypothesis. The Navier-Stokes equations can be written generically as Equation 2.1 together with continuity Equation 2.2 [FOX; MCDONALD, 1994].

$$
\begin{aligned}
\frac{D \mathbf{u}}{D t}-\frac{1}{2} \mu \nabla \cdot\left(\nabla \mathbf{u}+\nabla \mathbf{u}^{T}\right)+\nabla p & =\mathbf{f} \\
\nabla \cdot \mathbf{u} & =0
\end{aligned}
$$

where $\frac{D \mathbf{u}}{D t}=\frac{\partial \mathbf{u}}{\partial t}+\nabla \mathbf{u} \cdot \mathbf{u}$

When permanent regime is analyzed, then $\frac{D \mathbf{u}}{D t}=\frac{\partial \mathbf{y}^{0}}{\partial t}+\nabla \mathbf{u} \cdot \mathbf{u}$.

Transient regime can be considered in fluid diodes, however it does not bring too much value to the proposed analysis. Specially, when the diodes are inserted in equipment such as turbines and compressors without pulsating flow, the term $\frac{\partial \mathbf{u}}{\partial t}$ can be neglected, leading to Equation 2.3 and 2.4 . 


$$
\begin{aligned}
\nabla \mathbf{u} \cdot \mathbf{u}-\frac{1}{2} \mu \nabla \cdot\left(\nabla \mathbf{u}+\nabla \mathbf{u}^{T}\right)+\nabla p & =\mathbf{f} \\
\nabla \cdot \mathbf{u} & =0
\end{aligned}
$$

\subsubsection{Weak Formulation in 2D plane model}

For the problems where the time dependency is negligible, then in order to obtain the variational formulation and considering any external force equal to zero and $\mathbf{u} \in V, p \in Q$ and $\mathbf{w}=(\mathbf{u}, p)$, the strong formulation should be multiplied by test functions $(\mathbf{v}, q) \in W$ as shown in Equation 2.5.

$$
\int_{\Omega} \rho(\nabla \mathbf{u} \cdot \mathbf{u}) \cdot \mathbf{v} d \Omega+\int_{\Omega} \nabla p \cdot \mathbf{v} d \Omega-\frac{1}{2} \int_{\Omega} \mu \nabla \cdot\left(\nabla \mathbf{u}+\nabla \mathbf{u}^{T}\right) \cdot \mathbf{v} d \Omega=0
$$

Integrating Equation 2.5 by parts, it results in Equation 2.6. In this last form, the term in the boundary can be eliminate, beacause the boundary conditions to be applied considers a Dirichlet condition on the countour.

$\int_{\Omega} \rho(\nabla \mathbf{u} \cdot \mathbf{u}) \cdot \mathbf{v} d \Omega+\int_{\Omega} \nabla p \cdot \mathbf{v} d \Omega-\frac{1}{2} \int_{\Omega} \mu\left(\nabla \mathbf{u}+\nabla \mathbf{u}^{T}\right) \cdot \nabla \mathbf{v} d \Omega+\frac{1}{2} \int_{\partial \Omega} \mu\left(\nabla \mathbf{u}+\nabla \mathbf{u}^{T}\right) \cdot n \mathbf{v} \cdot d s=0$

Defining the weak form of the momentum equation as $R_{1}$ (Equation 2.7), continuity equation as $R_{2}$ (Equation 2.8) and combining them toghether in Equation 2.9.

$$
\begin{gathered}
R_{1}=\int_{\Omega} \rho(\nabla \mathbf{u} \cdot \mathbf{u}) \cdot \mathbf{v} d \Omega+\int_{\Omega} \nabla p \cdot \mathbf{v} d \Omega-\frac{1}{2} \int_{\Omega} \mu\left(\nabla \mathbf{u}+\nabla \mathbf{u}^{T}\right) \cdot \nabla \mathbf{v} d \Omega \\
R_{2}=\int_{\Omega} \nabla \cdot \mathbf{u} q \cdot d \Omega=0 \\
R_{1}+R_{2}=0
\end{gathered}
$$

The resulting system can written as Equation 2.10.

$$
\int_{\Omega} \rho(\nabla \mathbf{u} \cdot \mathbf{u}) \cdot \mathbf{v} d \Omega+\int_{\Omega} \nabla p \cdot \mathbf{v} d \Omega-\frac{1}{2} \int_{\Omega} \mu\left(\nabla \mathbf{u}+\nabla \mathbf{u}^{T}\right) \cdot \nabla \mathbf{v} d \Omega+\int_{\Omega} \nabla \cdot \mathbf{u} q \cdot d \Omega=0
$$




\subsubsection{Weak formulation in 2D Swirl Model}

It is the scope of this work to consider rotational speed in the generation of new topologies. Thus, a 2D axysimmetric model with swirl is implemented [ALONSO et al., 2018], which is commonly referred as 2.5D. Thus, the position and velocity vectors should be rewritten as shown in Equation 2.11 and 2.12.

Position:

$$
\mathbf{s}=(r, \theta, z)=r \mathbf{e}_{\mathbf{r}}+z \mathbf{e}_{\mathbf{z}}
$$

Velocity:

$$
\mathbf{u}=\left(u_{r}, u_{\theta}, u_{z}\right)=u_{r} \mathbf{e}_{\mathbf{r}}+u_{\theta} \mathbf{e}_{\theta}+u_{z} \mathbf{e}_{\mathbf{z}}
$$

Considering axisymmetry, the derivatives in relation to $\theta$ becomes zero $\left(\frac{\partial()}{\partial \theta}=0\right)$ With the system as shown in Figure 18, the velocities and acceleration equations are written as

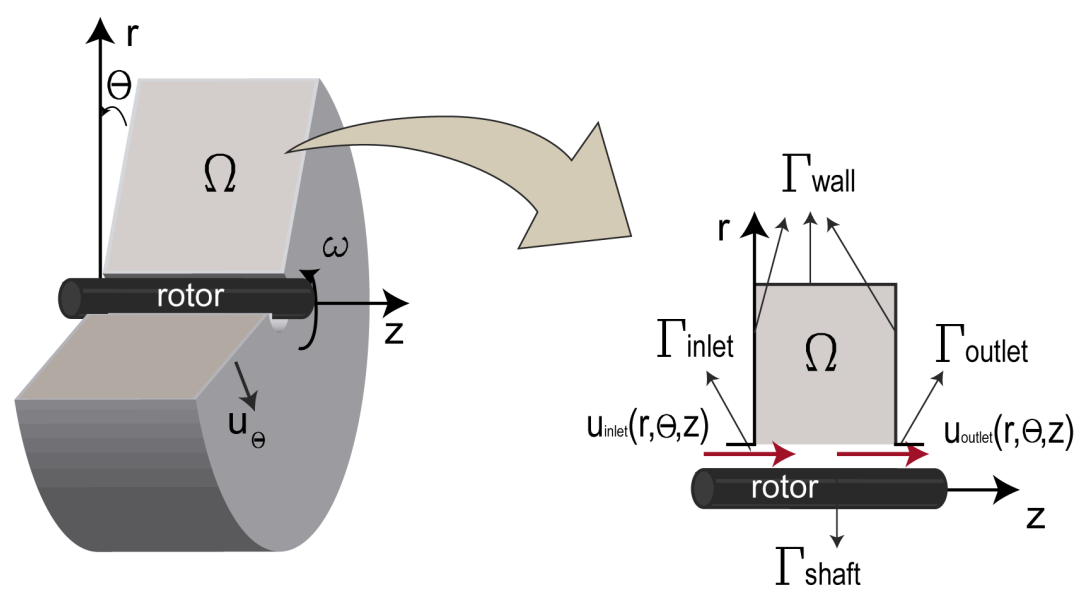

Figure 18: Coordinates System chosen to model the 2.5D System.

$$
\begin{gathered}
\mathbf{u}_{\mathbf{a b s}}=\mathbf{u}_{r}+\omega \wedge \mathbf{s} \\
\mathbf{a}_{\mathbf{a b s}}=\frac{D \mathbf{u}_{\mathbf{a b s}}}{D t}=\mathbf{a}+2 \omega \wedge \mathbf{u}_{r}+\omega \wedge(\omega \wedge \mathbf{s})+\hat{\omega} \wedge s
\end{gathered}
$$

When applying the rotating referential system to the Navier Stokes, the result is represented in Equation 2.15, which is the equation used in this project when 2D-Swirl is referred.

$$
\rho \nabla \mathbf{u}_{r} \cdot \mathbf{u}_{r}=-\nabla p+\mu \nabla^{2} \mathbf{u}_{r}+\rho \mathbf{f}-2 \rho \omega \wedge \mathbf{u}_{r}-\rho \omega \wedge(\omega \wedge s)
$$


Similar to previous section, the resulting equation is represented in Equation 2.16.

$$
\begin{array}{r}
\int_{\Omega} \rho\left(\nabla \mathbf{u}_{r} \cdot \mathbf{u}_{r}\right) \cdot \mathbf{v} d \Omega+\int_{\Omega} \nabla p \cdot \mathbf{v} d \Omega-\frac{1}{2} \int_{\Omega} \mu\left(\nabla \mathbf{u}_{r}+\nabla \mathbf{u}_{r}^{T}\right) \cdot \nabla \mathbf{v} d \Omega \\
-\int_{\Omega} \nabla \cdot \mathbf{u}_{r} q \cdot d \Omega \\
+\int_{\Omega} 2 \cdot \rho \omega \wedge \mathbf{u}_{r} \cdot \mathbf{v} d \Omega \\
+\int_{\Omega} \rho \cdot \omega \wedge(\omega \wedge s) \cdot \mathbf{v} d \Omega=0 \\
\frac{\partial()}{\partial \theta}=0
\end{array}
$$

with $d \Omega=2 \pi r d r d z$

\subsection{Finite Element Method for fluid Modelling}

This work uses the Taylor-Hood element, illustrated in Figure 19, for the finite element analysis, because it has been shown to be fast and easy to converge [TAYLOR; HOOD, 1973]. The idea of this approach is to use a triangular elements approximating the nodal velocities with 2- degree-polynomial and nodal pressure with 1-degree-polynomial, and the design variable is defined as one variable per element with discontinous Garlekin element (order 0 ).

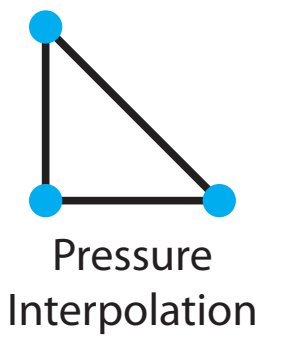

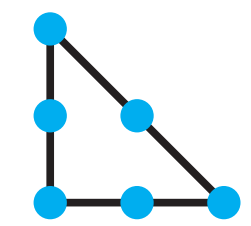

Velocity Interpolation

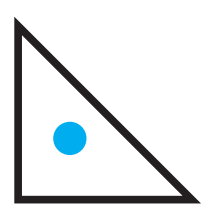

Design

Variable

Figure 19: Taylor Hood Element and its interpolation.

The triangular element (same with quadrilateral elements) illustrated in Figure 20 needs an interpolation done in local coordinate frame defined by $\epsilon$ and $\eta$ with $\Omega \in[0,1] \times$ $[0,1]$ and then a transformation to the global coordinate frame by using the relation with the vertices $\left(x_{1}, y_{1}\right),\left(x_{2}, y_{2}\right)$ and $\left(x_{3}, y_{3}\right)$.

$$
x=x_{1}+\left(x_{2}-x_{1}\right) \epsilon+\left(x_{3}-x_{1}\right) \eta
$$




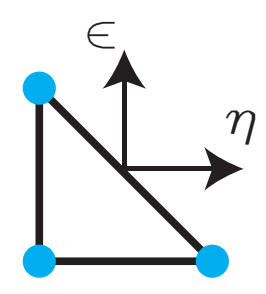

Figure 20: Triangular Element with coordinate frame.

$$
y=y_{1}+\left(y_{2}-y_{1}\right) \epsilon+\left(y_{3}-y_{1}\right) \eta
$$

By using the local coordinates, the interpolation for the pressure is given by

$$
\begin{gathered}
x_{1}(\epsilon, \eta)=1-\epsilon-\eta \\
x_{2}(\epsilon, \eta)=\epsilon \\
x_{3}(\epsilon, \eta)=\eta
\end{gathered}
$$

Analogously, the velocity is similar except for the quadratic interpolation

$$
\begin{gathered}
\psi_{1}(\epsilon, \eta)=(1-\epsilon-\eta)(1-2 \epsilon-2 \eta) \\
\psi_{2}(\epsilon, \eta)=\epsilon(2 \epsilon-1) \\
\psi_{3}(\epsilon, \eta)=\eta(2 \eta-1) \\
\psi_{4}(\epsilon, \eta)=4 \epsilon(1-\epsilon-\eta) \\
\psi_{5}(\epsilon, \eta)=4 \epsilon \eta \\
\psi_{6}(\epsilon, \eta)=4 \eta(1-\epsilon-\eta)
\end{gathered}
$$

After previous analyses it is necessary to perform the partition of the domain $\Omega$ into a finite set of cells such that together these cells form a mesh of the domain $\Omega$.

Then, it is necessary to substitute in the nonlinear variational problem considered in the analyzed case given by Equation 2.28.

$$
F(\mathbf{u} ; \mathbf{v})=0 \forall \mathbf{v} \in \hat{\mathrm{V}}
$$

where now $F: V \times \hat{\mathrm{V}} \rightarrow R$ is a semilinear form, linear in the argument(s) subsequent to 
the semicolon. With the discretization of the variational problem restricted to a pair of discrete trial and test spaces: find $\mathbf{u}_{h} \in V_{h} \subset V$ satisfying Equation 2.29.

$$
F\left(\mathbf{u}_{h} ; \mathbf{v}\right)=0 \forall v \in \hat{\mathrm{V}}_{h} \subset \hat{\mathrm{V}}
$$

The finite element solution $\mathbf{u}_{h}=\sum_{j=1}^{N} \mathbf{U}_{j} \Phi_{j}$ may be calculated by solving a nonlinear system .

$$
\mathbf{b}(\mathbf{U})=0
$$

where $\mathbf{b}: \mathbb{R}^{N} \rightarrow \mathbb{R}^{N}$ and $\mathbf{b}_{i}(\mathbf{U})=F\left(\mathbf{u}_{h}, \hat{\phi}\right)$

In order to solve the nonlinear system, the Newton method is used by computing the Jacobian $\mathbf{A}=\mathbf{b}^{\prime}$. If the semilinear form $\mathrm{F}$ is differentiable in $\mathbf{u}$, then the entries of the Jacobian $\mathbf{A}$ are given by Equation 2.31.

$$
\mathbf{A}_{i j}\left(\mathbf{u}_{h}\right)=\frac{\partial b_{i}(\mathbf{U})}{\partial \mathbf{U}_{j}}=\frac{\partial}{\partial \mathbf{U}_{j}} F\left(\mathbf{u}_{h}, \hat{\phi}\right)=F^{\prime}\left(\mathbf{u}_{h}, \hat{\phi}_{i}\right) \frac{\partial \mathbf{u}_{h}}{\partial \mathbf{U}_{j}}=F^{\prime}\left(\mathbf{u}_{h}, \hat{\phi}_{i}\right) \Phi_{j}=F^{\prime}\left(\mathbf{u}_{h}, \Phi_{j}, \hat{\phi}_{i}\right)
$$

At each Newton iteration, it is necessary then to evaluate (assemble) the matrix $\mathbf{A}$ and update the solution vector $\mathbf{U}$ by

$$
\mathbf{U}^{k+1}=\mathbf{U}^{k}-\delta \mathbf{U}^{k}, k=0,1, \ldots
$$

with $\partial \mathbf{U}^{k}$ to solve the linear system 2.33.

$$
\mathbf{A}\left(\mathbf{u}_{h}^{k}\right) \partial \mathbf{U}^{k}=\mathbf{b}\left(\mathbf{u}_{h}^{k}\right)
$$

At each Newton iteration, a linear variational problem of the canonical form is solved and the discretization of Equation 2.34 recovers the linear system.

$$
F^{\prime}\left(\mathbf{u}_{h} ; \partial \mathbf{u}, \mathbf{v}\right)=F\left(\mathbf{u}_{h} ; \mathbf{v}\right) \quad \forall \mathbf{v} \in \hat{\mathbf{V}}_{h}
$$




\section{TOPOLOGY OPTIMIZATION METHOD}

The traditional topology optimization method used in fluids are based on density method [BORRVALL; PETERSSON, 2003] , in which design variables from a design domain can reach values between 0 (solid) and 1 (fluid).

In the current implementation of topology optimization for fluids considering density methods, there are essentially three problems. First, the gray scale in the result turns difficult the identification of the contour of the fluid mesh, which may be a problem in some applications also during the optimization process. Second, even for low Reynolds flow design problems, a continuation scheme of the material model penalization parameters is necessary to avoid gray scale and to obtain clear boundaries. Third, in complex fluid flow optimization problems it is difficult to specify the maximum value of the inverse permeability in order to avoid the fluid flow inside the solid.

For this reason, this work proposes a novel methodology that tackles the first two problems, i.e., it avoids gray scale and obtain clear boundaries. The idea is to implement the Topology Optimization of Binary Structures (TOBS) [NADA, c] for fluid flow design, which is a novel topology optimization method that has been used in solid mechanics to generate optimized structural solutions considering only binary $\{0,1\}$ design variables. The main advantage of $\{0,1\}$ methods is the clear definition of the interface and the absence of gray scale. It is a method easy to implement which preserves the material distribution features.

Thus, this section explains the material model, objective functions and the TOBS method applied to fluids.

\subsection{Material Model for Topology Optimization}

In this research, the approach [BORRVALL; PETERSSON, 2003; GERSBORG-HANSEN; SIGMUND; HABER, 2005] is to adopt a field of porosity to determine the path to be 
covered by the fluid. Regions of low permeability are considered to be solid and regions of high permeability are considered to be fluid. The variable used in this project is the inverse permeability $(\alpha)$.

It is important to interpolate the material flow (see Figure 21) in the domain and an absorption coefficient $\kappa$ is defined, which in solid regions it has values of $\kappa>>1$ and in fluid regions it has values of $\kappa=0$. For the optimization algorithm of fluid diodes, the material model function of Equation 3.1 is used [BORRVALL; PETERSSON, 2003].

$$
\kappa(\alpha)=\kappa_{\max }+\left(\kappa_{\min }-\kappa_{\max }\right) \alpha \frac{1+q}{\alpha+q}
$$

where $\kappa \in\left[\kappa_{\min }, \kappa_{\max }\right]$ and $q$ is the parameter that controls the linearity of $\kappa$. Thus, when $q \rightarrow \infty, \kappa \rightarrow \kappa_{\max }\left(\kappa_{\min }-\kappa_{\max }\right)$ becomes a linear function.

A relevant difference when appliying the TOBS method is that the $q$ factor is set to be 1 and does not need to change during the optimization. Also, a linear form of material model interpolation could be used, however, it is kept the traditional form in order to facilitate comparisons.

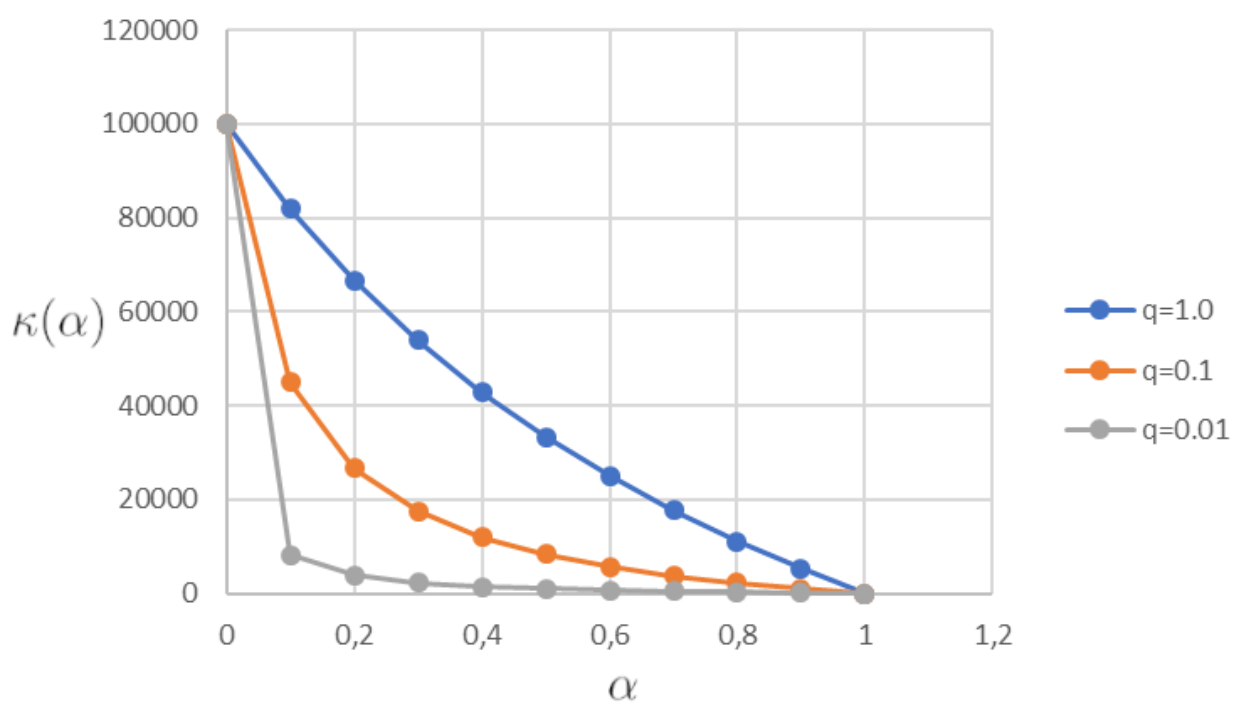

Figure 21: Plot of the relationship between the $\mathrm{q}, \alpha$ and $\kappa$ parameters based on [BORRVALL; PETERSSON, 2003].

\subsection{Objective Functions}

This section start with common used objective functions and then explores properly different possible alternatives to the fluid diodes design problem. 


\subsubsection{Total Pressure Loss}

The first idea of an objective function could be the maximization of the pressure drop between inlet and outlet in a certain design domain. The effectiveness of a particular seal design or configuration can be represented in terms of the loss of total pressure across the seal. The pressure loss can be expressed, relative to the inlet total pressure, in dimensionless form as given by Equation 3.2.

$$
\Delta P=\frac{P_{\text {inlet }}-P_{\text {outlet }}}{P_{\text {inlet }}}
$$

This approach could be very useful because it is a direct measure of the relative energy dissipated in the seal internal flow passages, however, when considered as objective function this approach did not converge easily and for this reason other objective functions are explored.

\subsubsection{Energy Dissipation}

The most common objective function used in topology optimization in fluids is energy dissipation inside a design domain. [BORRVALL; PETERSSON, 2003] defined in Equation 3.3.

$$
\Phi(\boldsymbol{u}, \alpha)=\frac{1}{2} \mu \int_{\Omega}\left\|\nabla \boldsymbol{u}+\nabla \boldsymbol{u}^{T}\right\|^{2} d \Omega+\int_{\Omega} \kappa(\alpha)\|\boldsymbol{u}\|^{2} d \Omega
$$

where $\mathbf{u}$ is velocity vector, $\kappa(\alpha)$ the inverse porosity of an element (fluid or structure) and $\mu$ the viscosity.

The first term of Equation 3.3 can be sometimes simplified in a 2D scenario as shown in Equation 3.4 [LIN et al., 2015].

$$
\left\|\nabla \boldsymbol{u}+\nabla \boldsymbol{u}^{T}\right\|^{2}=\mu\left(\partial_{i} u_{i}+\partial_{j} u_{j}\right) \partial_{j} u_{i}=\sum \mu\left(\partial_{i} u_{i}+\partial_{j} u_{j}\right)^{2}
$$

The problem with this function is that when working with labyrinth seals its maximization usually brings results blocking the inlet and outlet. That is completely undesirable since rotating parts requires a distance from stationary parts. This is illustrated in Figure 22. 


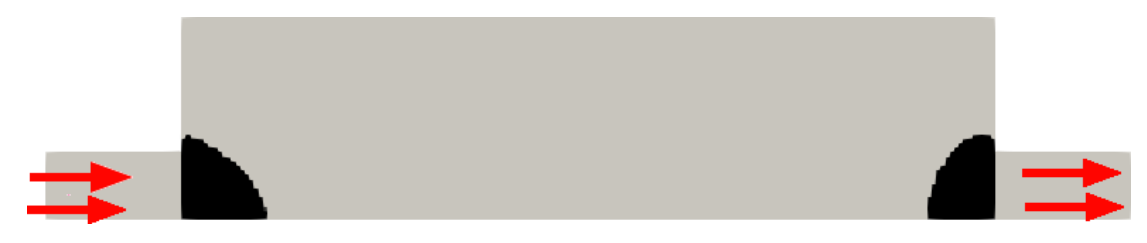

Figure 22: Result of maximizing energy dissipation in a labyrinth seal (black: solid, white: fluid).

\subsubsection{Diodicity}

In order to fix the previous issue, an approach of considering labyrinth seals as fluid diodes are used. In fluid diodes design the usual adopted objective function is the diodicity. The diodicity is defined in Equation 3.5 as a relation between the pressure difference between two flows (forward and reverse). The analysis considers both forward and reverse flow illustrated in Figure 23.

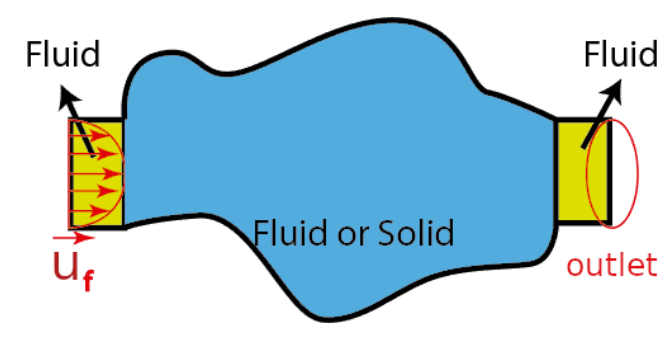

(a) Forward Flow

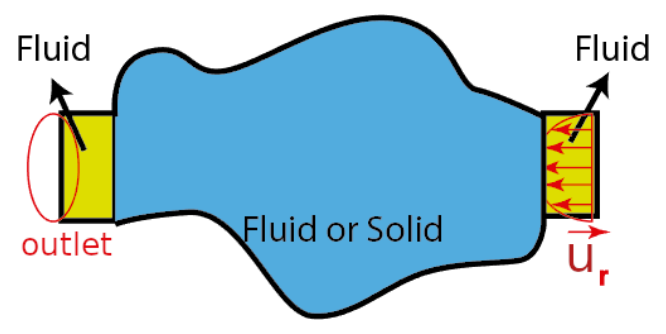

(b) Reverse Flow

Figure 23: Fluid flow load cases.

$$
D i=\frac{\Delta p_{\text {reverse }}}{\Delta p_{\text {forward }}}
$$

[LIN et al., 2015] shows that the relationship between pressure drop is equivalent of relationship of energy dissipation (see Equation 3.6).

$$
D i=\frac{\Delta p_{\text {reverse }}}{\Delta p_{\text {forward }}}=\frac{\Delta p_{r} \cdot Q_{r}}{\Delta p_{f} \cdot Q_{f}}=\frac{\Phi\left(u_{r}, p_{r}\right)}{\Phi\left(u_{f}, p_{f}\right)}
$$

where $Q$ is the fluid flow given by $Q=\int \mathbf{u} d S$.

When simulating diodicity, the nonlinear optimization problem has a variety of solutions, thus a term $F$ with its weight $W_{F}$ (usually from 0.1 to 3 ) should be added to the objective function and configured to avoid undesirable local minima. The idea of $F$ is to enable the reverse flow to occur, minimizing $\left(\kappa \cdot\|\mathbf{u}\|_{\text {reverseFlow }}^{2}\right)$ term, caring not to 
influence very much the main objective function (diodicity).

$$
F=\frac{1}{L^{2}} \int_{\Omega} \frac{\kappa \cdot\|\mathbf{u}\|_{\text {reverseFlow }}^{2}}{\kappa_{\max } \mathbf{U}_{\text {reverseAverage }}} d \Omega
$$

where $L$ represents the inlet size.

Thus, the problem of a fluid diode can be set as Equation 3.8. However, this proposed optimization problem does not solve two common problems, which are velocity through solid parts (see Fig. 24a) and floating islands in the design domain (see Fig. 24b).

$$
\begin{aligned}
& \min _{\alpha} \frac{\Phi\left(\mathbf{u}_{f}, p_{f}\right)}{\Phi\left(\mathbf{u}_{r}, p_{r}\right)}+W_{F} \cdot F \\
& \text { s.t. }(u \cdot \nabla) \mathbf{u}-\mu \nabla^{2} \mathbf{u}+\nabla p+\alpha \cdot \mathbf{u}=0 \quad \text { in } \Omega \\
& \nabla \cdot \mathbf{u}=0 \quad \text { in } \Omega \\
& V_{\text {down }} \leq \int \alpha d \Omega \leq V_{\text {up }} \\
& 0 \leq \alpha \leq 1
\end{aligned}
$$

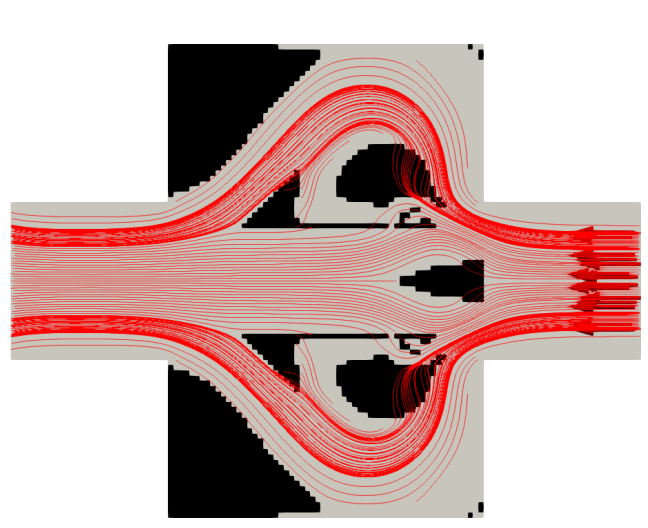

(a) Fluid flowing through solids.

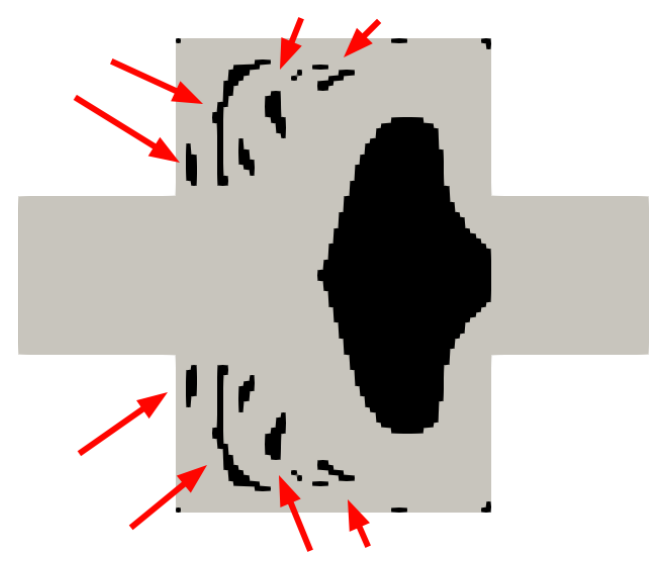

(b) Undesirable floating islands.

Figure 24: Issues with the proposed problem with simple diodicity.

In a 2D plane fluid diode such geometries are acceptable, however, labyrinth seals with rotational conditions having floating islands may not be wanted, thus to solve previous problems, structural terms under forces from fluid are considered. It introduces an additional term in the objective function similar to fluid-solid interaction problems, trying to minimize the compliance of structures (solid parts) as described in Section 3.2.5. 


\subsubsection{Vorticity}

A novel proposed approach to design fluid diodes is to consider the vorticity squared (defined in Equation 3.9) of the flow which is desirable to raise inside the cavity in order to recirculate fluid and inhibit flow in a certain direction.

Such approach is necessary because diodicity alone may not bring interesting and complex geometries to the results. Vorticity induces rotational flow in the labyrinth seal and, consequently, more complex and efficient geometry are generated.

$$
\operatorname{Vor}(\mathbf{u}, p)=\int_{\Omega}(\nabla \times \mathbf{u}):(\nabla \times \mathbf{u}) d \Omega
$$

The optimization problem considering vorticity could be described by Equation 3.10.

$$
\begin{array}{ccc}
\max & \operatorname{Vor}_{r}\left(\mathbf{u}_{r}, p_{r}\right)=\int_{\Omega}\left(\nabla \times \mathbf{u}_{r}\right):\left(\nabla \times \mathbf{u}_{r}\right) d \Omega & \\
\text { s.t. } & (u \cdot \nabla) \mathbf{u}-\mu \nabla^{2} \mathbf{u}+\nabla p+\alpha \cdot \mathbf{u}=0 & \text { in } \Omega \\
\nabla \cdot \mathbf{u}=0 & \text { in } \Omega \\
V_{\text {down }} \leq \int \alpha d \Omega \leq V_{u p} & \\
0 \leq \alpha \leq 1 &
\end{array}
$$

The main goal of this work is to raise the vorticity in the real flow direction in order to generate complex and interesting geometries. Thus, this term is added to the full optimization problem, considering the reverse flow (from right to left), however with a minus signal since the original optimization problem is minimization. Thus, the complete problem of a fluid diode can be described in Equation 3.11.

$$
\begin{aligned}
& \min _{\alpha} \frac{\Phi\left(\mathbf{u}_{f}, p_{f}\right)}{\Phi\left(\mathbf{u}_{r}, p_{r}\right)}+W_{F} \cdot F-W_{V} \cdot \text { Vor }_{r} \\
& \text { s.t. }(u \cdot \nabla) \mathbf{u}-\mu \nabla^{2} \mathbf{u}+\nabla p+\alpha \cdot \mathbf{u}=0 \quad \text { in } \Omega \\
& \nabla \cdot \mathbf{u}=0 \quad \text { in } \Omega \\
& V_{\text {down }} \leq \int \alpha d \Omega \leq V_{\text {up }} \\
& 0 \leq \alpha \leq 1
\end{aligned}
$$




\subsubsection{Fluid-Structure interaction (FSI)}

As explained in previous section undesirable islands in some cases may remain in the final solution. For this reason, a compliance term of the solid parts is added to the objective function and the forces can come from the fluid flow simulation.

The main idea is illustrated in Figure 25 showing that the pressure field obtained from a CFD simulation is transformed into forces on structures (solid parts) with a boundary condition of no displacements on the boundaries and this tends to eliminate floating islands since the deformation is high when not attached to wall. One additional hypothesis in this simulation is that the deformation of the structure is too small to interfere in the fluid flow simulation.

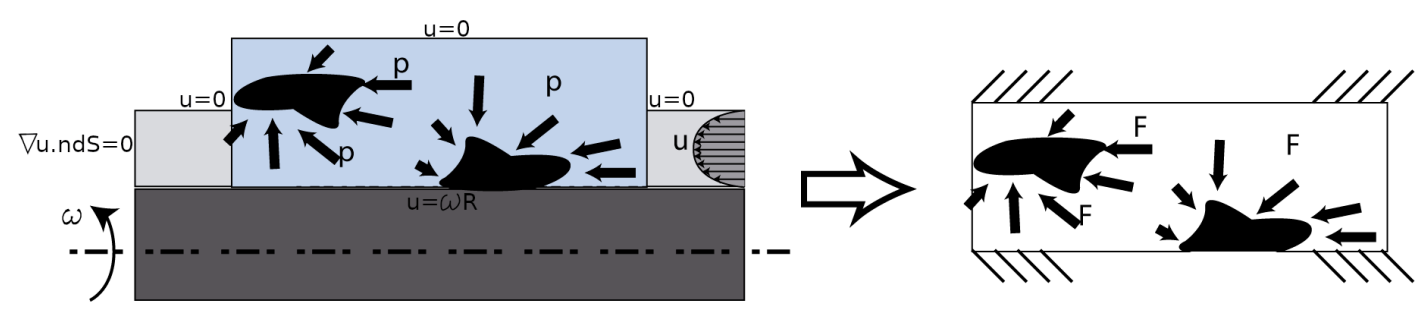

Figure 25: Scheme showing how forces and boundary conditions are applied on structures with $\mathbf{u}$ as velocity with parabolic profile on inlet with magnitude according to a Reynods number value.

After applying properly the forces on solids, the structural term must be introduced in the objective function as minimization of the compliance (strain energy), defined in Equation 3.12. The structure analysis in this work is performed, considering isotropic material with Young's Moduls of 200GPa and Poisson's ratio of 0.3.

$$
E=\int \frac{1}{2} \boldsymbol{\sigma}: \boldsymbol{\epsilon} d \Omega
$$

where $\boldsymbol{\sigma}$ is mechanical stress tensor and $\boldsymbol{\epsilon}$ is symmetric strain-rate tensor of structure.

To solve the forward problem of structural problem in the variation formulation, the Equation 3.13 is necessary to be solved.

$$
\int \boldsymbol{\sigma}(x): \boldsymbol{\epsilon}(v) d \Omega=0
$$


with $\mathrm{x}$ as displacement, and $\mathrm{v}$ as test function

The structural term is added in the full optimization problem in Equation 3.14, with a term $W_{E}$ to properly scale to a similar order of magnitude of $D i$.

$$
\begin{array}{ccc}
\min & \frac{\Phi\left(\mathbf{u}_{f}, p_{f}\right)}{\Phi\left(\mathbf{u}_{r}, p_{r}\right)}+W_{F} \cdot F-W_{V} \cdot \text { Vor }_{r}+W_{E} \cdot E & \\
\text { s.t. } & (u \cdot \nabla) \mathbf{u}-\mu \nabla^{2} \mathbf{u}+\nabla p+\alpha \cdot \mathbf{u}=0 & \text { in } \Omega \\
\nabla \cdot \mathbf{u}=0 & \text { in } \Omega \\
\int \sigma(x): \epsilon(v) d \Omega=0 & \\
V_{\text {down }} \leq \int \alpha d \Omega \leq V_{u p} & \\
0 \leq \alpha \leq 1 &
\end{array}
$$

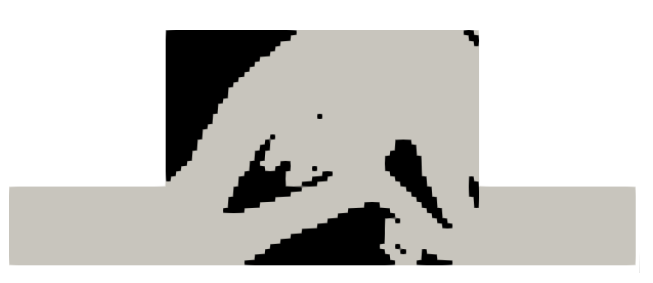

(a) Labyrinth seal with undesirable islands.

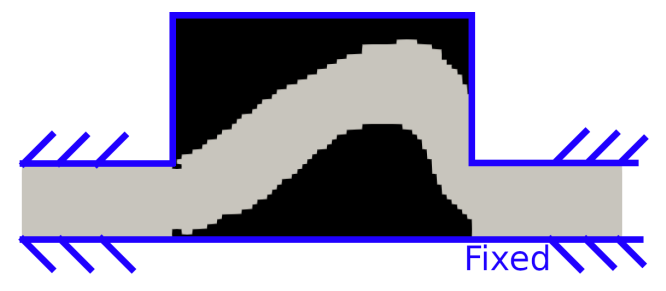

(b) FSI term added to the objective function.

Figure 26: Results when considering structural terms or not.

The benefits of adding such term is illustrated in Figure 26 a-b, where floating islands disappear and the geometry becomes clean.

\subsubsection{Filtering in topology optimization}

Fluid flow simulation usually does not require the use of filter in the topology optimization, because it usually does not present checkerboard patterns or mesh dependency. However, the maximization of energy dissipation or the use of diodicity bring results with some challenges, such as small width channels which require some kind of complexity control for minimum size.

The filter employed in this work is the same of [GUEST; GENUT, 2010], since the idea is to separate the design variable discretization from the mesh. For that, a Heaviside projection scheme can be defined in Equation 3.15. 


$$
\begin{gathered}
\phi(\alpha)=\left\{\begin{array}{c}
0 \text { if } \sum_{i \in N^{e}} \alpha_{i}>0 \\
1 \text { if } \sum_{i \in N^{e}} \alpha_{i}=0
\end{array}\right. \\
i \in N^{e} \text { if }\left\|x_{i}-x_{e}\right\|<r_{\text {min }}
\end{gathered}
$$

where $\phi(\alpha)$ is filtered design variable, $x_{i}$ and $x_{e}$ are the position of an element from the mesh to an element of design variable, respectively, and the distance between them should be inside a distance $r_{\min }$ (see Figure 27).

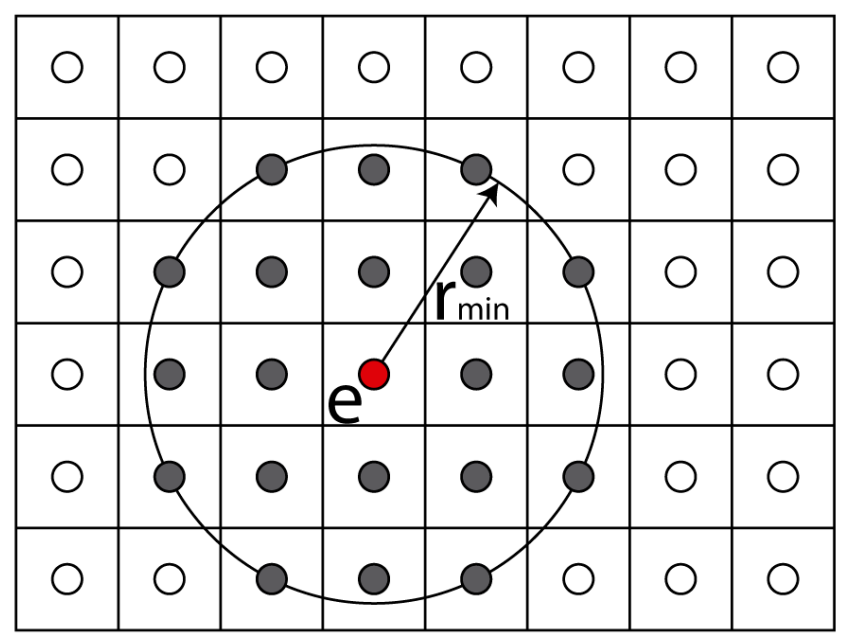

Figure 27: Elements $i$ of a Neighboorhood $\left(N^{e}\right)$ inside a distance of $r_{\min }$ to an element $e$.

\subsection{The TOBS Method}

In the TOBS methodology [NADA, $\mathrm{c}, \mathrm{d}$ ], a set of binary variables $\alpha_{j} \in\{0,1\}$ is defined to indicate the absence or the presence of material and in this work it is related to the permeability of the fluid. A generic binary optimization problem with inequality constraints is given by Equation 3.16.

$$
\begin{aligned}
& \underset{\boldsymbol{\alpha}}{\operatorname{Minimize}} f(\boldsymbol{\alpha}) \\
& \text { Subject to } g_{i}(\boldsymbol{\alpha}) \leq \bar{g}_{i}, i \in\left[1, N_{g}\right] \\
& \qquad \alpha_{j} \in\{0,1\}, j \in\left[1, N_{d}\right]
\end{aligned}
$$

where $\boldsymbol{\alpha}$ is the vector of design variables (usually called densities in the case of structural Topology Optimization) of size $N_{d}, f$ is the objective function, $g_{i}$ is the $i^{\text {th }}$ inequality 
constraint with $\bar{g}_{i}$ being its associated upper bound and $N_{g}$ is the number of inequality constraints.

In the TOBS method the optimization problem is approximated by Equation 3.17, a sequential linearization of the objective and constraint functions. Thus, the approximated linearized optimization problem at an iteration $k$ is represented by

$$
\begin{aligned}
\underset{\Delta \boldsymbol{\alpha}^{k}}{\operatorname{Minimize}} & \frac{\partial f\left(\boldsymbol{\alpha}^{k}\right)}{\partial \boldsymbol{\alpha}} \cdot \Delta \boldsymbol{\alpha}^{k} \\
\text { Subject to } & \frac{\partial g_{i}\left(\boldsymbol{\alpha}^{k}\right)}{\partial \boldsymbol{\alpha}} \cdot \Delta \boldsymbol{\alpha}^{k} \leq \bar{g}_{i}-g\left(\boldsymbol{\alpha}^{k}\right)=\Delta g_{i}^{k}, i \in\left[1, N_{g}\right] \\
& \left\|\Delta \boldsymbol{\alpha}^{k}\right\|_{1} \leq \beta N_{d} \\
& \Delta \alpha_{j}^{k} \in\left\{-\alpha_{j}^{k}, 1-\alpha_{j}^{k}\right\}, j \in\left[1, N_{d}\right]
\end{aligned}
$$

where $(\cdot)^{k}$ indicates the quantity $(\cdot)$ at iteration $k, \Delta \boldsymbol{\alpha}^{k}$ is the update values of design variables and $\Delta g_{i}^{k}$ is the upper bound of constraint $i$. To avoid drastic changes of the design, an extra-constraint based on the 1-norm of design variables is added to restrict the number of flips from 0 to 1 and vice-versa. A parameter $\beta$ defines a percentage of the total number $N_{d}$ of design variables that is allowed to flip variables. By using small values of $\beta$ ensures that the number of flips remains low at each iteration $k$, thereby keeping the truncation error $O\left(\left\|\Delta \boldsymbol{\alpha}^{k}\right\|_{2}^{2}\right)$ of the linearization approximation small. Such tool is very important in structural problems, however, not very useful to fluid flow problems, as shown in the results. Since this study can work in both fields the $\beta$ stands in Equation 3.17 .

The volume constraint in TOBS method uses $\epsilon$ value that restricts big changes in the design variables over optimization iterations. This is discussed in numerical examples, however what matters in practice in fluids is how fast the optimization goes to a local minimum, because the range of the limits in the linearized optimization is higher. This behaviour can be seen as example in Figure 52 of result section. The constraint bounds are modified by using Equation 3.18.

$$
\Delta g_{i}^{k}= \begin{cases}-\varepsilon_{1} g_{i}\left(\boldsymbol{\alpha}^{k}\right) & : \bar{g}_{i}<\left(1-\varepsilon_{1}\right) g_{i}\left(\boldsymbol{\alpha}^{k}\right) \\ \bar{g}_{i}-g_{i}\left(\boldsymbol{\alpha}^{k}\right) & : \bar{g}_{i} \in\left[\left(1-\varepsilon_{1}\right) g\left(\boldsymbol{\alpha}^{k}\right),\left(1+\varepsilon_{2}\right) g\left(\boldsymbol{\alpha}^{k}\right)\right] \\ \varepsilon_{2} g_{i}\left(\boldsymbol{\alpha}^{k}\right) & : \bar{g}_{i}>\left(1+\varepsilon_{2}\right) g_{i}\left(\boldsymbol{\alpha}^{k}\right)\end{cases}
$$

The integer optimization problem comes from sequential linearization that can be 
solved via Integer Linear Programming (ILP). In this work, the ILP problem is solved by using the branch-and-bound solver present in $\mathrm{CPLEX}^{\circledR}$ optimization package from $\mathrm{IBM}^{\circledR}$. Other libraries have been also tested, such as Mixed-integer linear programming (MILP) from Matlab ${ }^{\circledR}$ and glpk from Octave, however, they all lack of efficiency.

The branch-and-bound method is an algorithm based on the heap data structure. The ILP is first solved without any integer constraints using some linear optimization techniques. Then branches of the problem are created with additional inequality constraints on the design variables in order to find integer solutions. This process repeats until the first integer solution is obtained.

\subsection{Movable rotation boundary condition (MRBC)}

In some design application, it is desirable to have solid regions attached to the $\operatorname{shaft}\left(\omega=\frac{u_{\theta}}{r}\right)$ rather than the stator $(\omega=0)$ (see Figure 28).

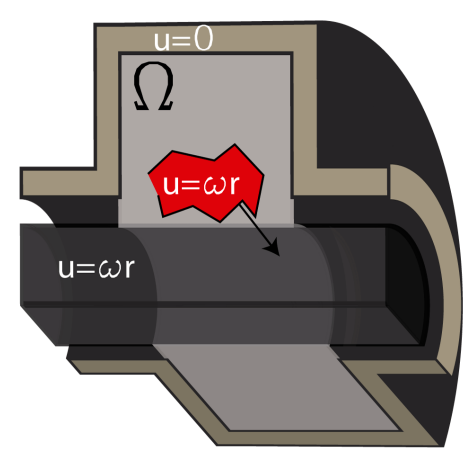

(a) Solid (red) attached to the shaft.

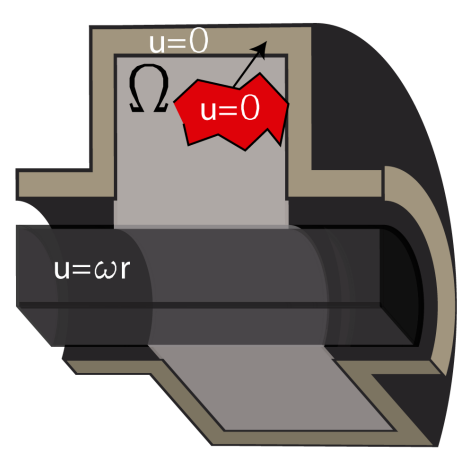

(b) Solid (red) attached to the stator.

Figure 28: Solid position and rotational speed.

Thus, a movable rotation boundary condiction (MRBC) has to be implemented, however, the difficult of the MRBC relies on how to determine where each solid part belongs to, along the optimization process. It could be set, for example, a parameter as the distance from the centroid of the solid parts to the shaft or stator, however this would have a problem with solids in the middle of the design domain.

For this reason, it is implemented an algorithm able to transform a generic 2D point cloud into polygons that can be extracted and meshed. The main idea is to use 2D Delaunay triangulation and then to extract geometries. The goal is to maximize the smaller angle of a triangle using the point cloud and then determine a mesh that can 
define a surface. Figure 29 shows an example to determine if D belongs to the circle or not, and then select the points of the mesh. This determination can be done by using the determinant of Equation 3.19.

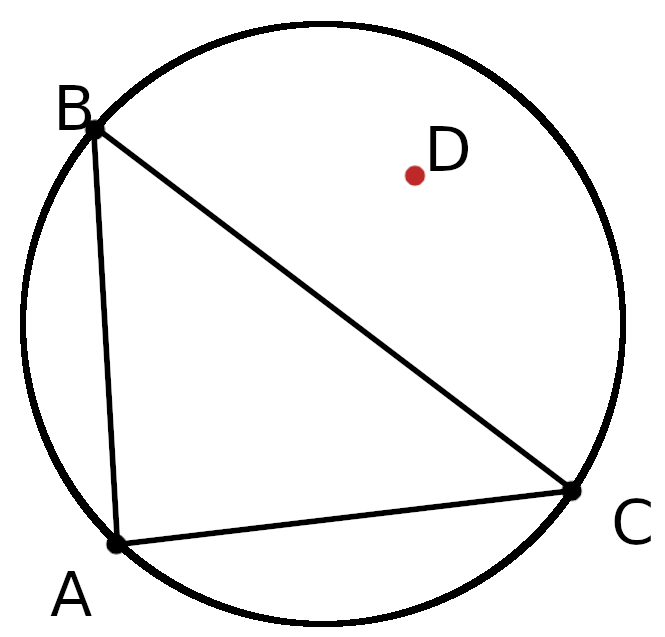

Figure 29: Delaunay algorithm to determine if a point belongs to a circle.

$$
\left|\begin{array}{lll}
A_{x}-D_{x} & A_{y}-D_{y} & \left(A_{x}-D_{x}\right)^{2}+\left(A_{y}-D y\right)^{2} \\
B_{x}-D_{x} & B_{y}-D_{y} & \left(B_{x}-D_{x}\right)^{2}+\left(B_{y}-D y\right)^{2} \\
C_{x}-D_{x} & C_{y}-D_{y} & \left(C_{x}-D_{x}\right)^{2}+\left(C_{y}-D y\right)^{2}
\end{array}\right|>0 \Leftrightarrow D \in \text { Circle }
$$

where indexes $x$ and $y$ indicate the coordinate of each point.

Figure 30 shows the process, starting with a point cloud,that is obtained after a topology optimization step, followed by a delaunay triangulation, determining the correct triangles to be considered. Finally, it is determined two different polygon (green or blue) and also a hole in each surface when appropriate.

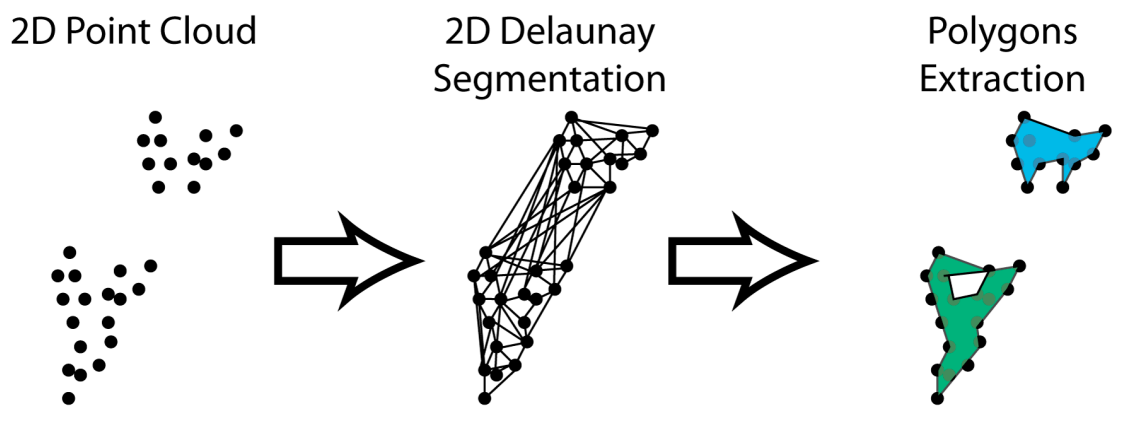

Figure 30: Classification algorithm detecting geometries.

Now, to properly implement the MRBC, the points on the surface of the shaft are 
forced to exist and when some design variables are turned into solid and they are near the shaft, the classification algorithm will determine these points as an unique geometry and as a shaft. After this step, the boundary condition with rotational velocity is applied. All solids considered far by the triangulation algorithm from the shaft will receive a different polygon and will receive a zero velocity boundary condition. All the process is illustrated in Figure 31.

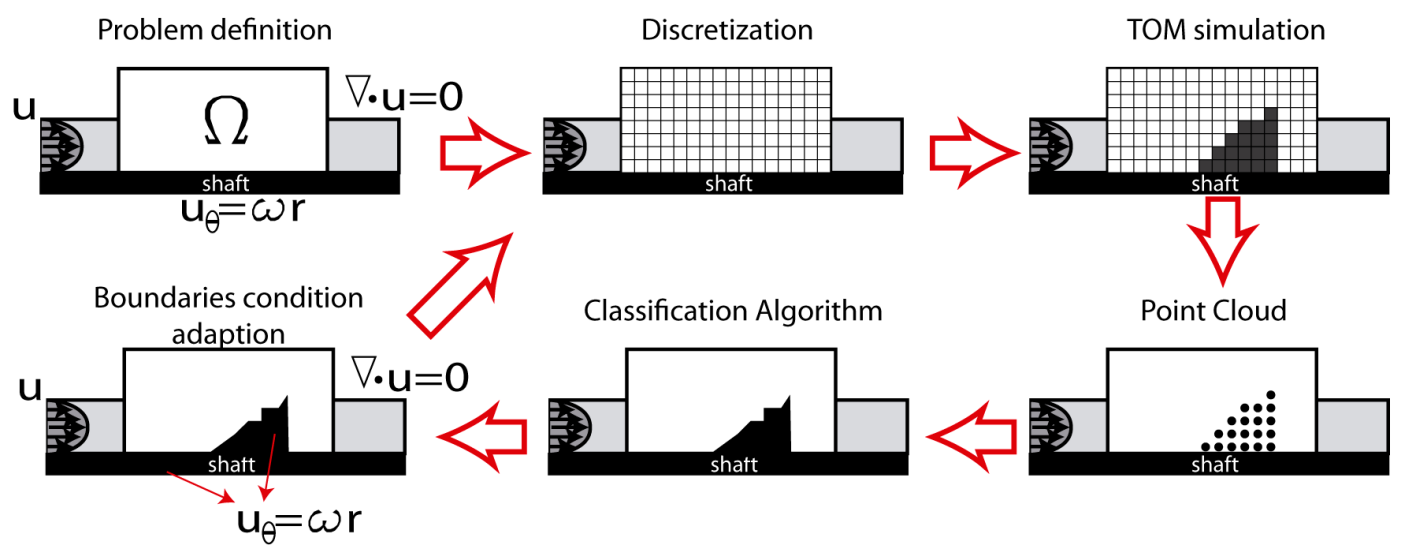

Figure 31: Rotation selection using classification for new geometries during TO process.

Figure 32 shows that the algorithm can detect properly new geometries attached to shaft during optimization process.

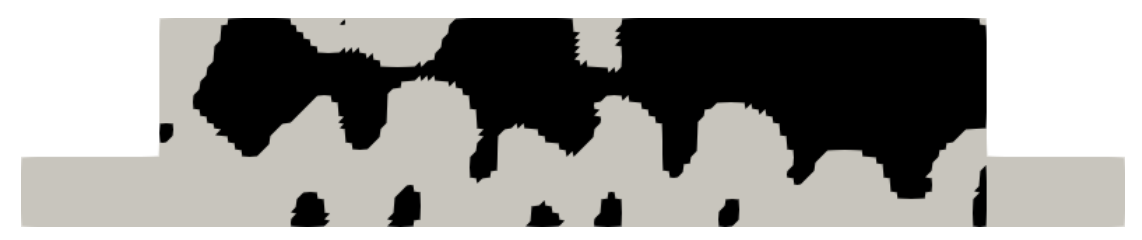

(a) Generic geometry obtained with topology optimization in a 2D swirl model.

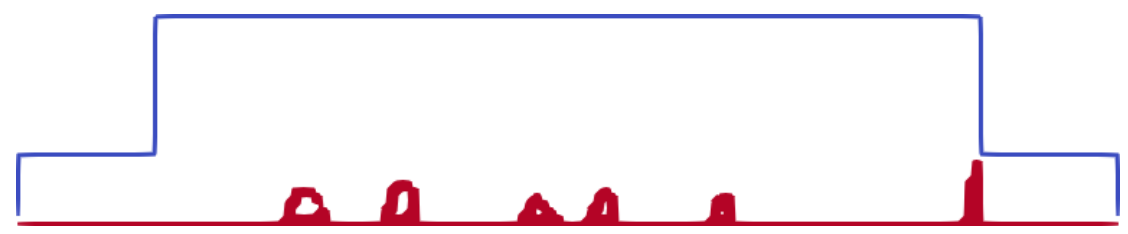

(b) Rotational velocity applied to the red region since it may be easily attached to the shaft

Figure 32: Classification algorithm used to design fluid diodes.

Fluid flow simulation usually requires the exactly determination of the contour of the fluid domain to correctly discretize, however it is not elementary to do it when having gray scale in the topology optimization result and classic topology optimization methods requires gray scale. For this reason the Topology Optmization of Binary Structures (TOBS) 
[SIVAPURAM; PICELLI, 2018; SIVAPURAM; PICELLI; XIE, 2018] was extended to be used with fluids and applied to this work.

\subsection{Sensitivity Analysis}

In this work, the objective function can have several sensitivity terms: diodicity, fluid vorticity and also structural compliance. The full sensitivity is validated in Appendix C to check if the optimization has the correct gradient.

Therefore, for each term of the objective function, the following analysis can be done, considering a functional $J(\alpha, \mathbf{u})$. The final optimization problem can be represented in Equation 3.20.

$$
\begin{array}{lll}
\min & J(\alpha, \mathbf{u}) & \\
\alpha & & \\
\text { s.t. } & (u \cdot \nabla) \mathbf{u}-\mu \nabla^{2} \mathbf{u}+\nabla p+\alpha \cdot \mathbf{u}=0 & \text { in } \Omega \\
& \nabla \cdot \mathbf{u}=0 & \text { in } \Omega \\
& \mathbf{u}=\mathbf{u}(z) & \text { on }\left[\partial \Omega_{\text {inlet }}\right] \\
& \mathbf{u}=0 & \text { on }\left[\partial \Omega_{\text {wall }}\right]
\end{array}
$$

The gradients of the problem plays an important role in the optimization, therefore it is now written the adjoint system of the Navier Stokes Equation. The definition of the lagrangian is in Equation 3.25 with the terms that will be calculate separately later $\left(L_{1}, L_{2}, \ldots\right)$ in Equation 3.26.

$$
\frac{d J}{d \alpha}=\underbrace{\frac{\partial J}{\partial \mathbf{u}} \frac{\partial \mathbf{F}^{-1}}{\partial \mathbf{u}}}_{\lambda} \frac{\partial \mathbf{F}}{\partial \alpha}+\frac{\partial J}{\partial \alpha}
$$

where $\mathbf{F}$ represents Equation 3.21 and 3.22.

$$
\begin{gathered}
L:=\frac{\partial J}{\partial \alpha}-\int_{\Omega} \lambda \cdot(\overbrace{(\mathbf{u} \cdot \nabla) \mathbf{u}-\mu \nabla^{2} \mathbf{u}}^{L_{1}}+\overbrace{\nabla p}^{L_{3}}+\overbrace{\alpha \cdot \mathbf{u}}^{L_{2}}) d \Omega \\
-\int_{\Omega} \beta \overbrace{\nabla \cdot \mathbf{u}}^{L_{5}} d \Omega-\int_{\partial \Omega_{\text {inlet }}} \gamma \cdot(\mathbf{u}-m) d \Omega-\int_{\partial \Omega_{\text {wall }}} \rho \cdot(\mathbf{u}) d \Omega
\end{gathered}
$$

with $\lambda, \beta, \gamma, \rho$ as lagrangian multipliers 
Using the Frechet derivatives, it is possible to derive all the terms $L_{1}, L_{2}, L_{3}, L_{4}, L_{5}$, as:

$$
\begin{aligned}
& L_{1}^{\prime}=\lim _{\epsilon \rightarrow 0+} \frac{1}{\epsilon} \int_{\Omega}([(\mathbf{u}+\epsilon \mathbf{v}) \cdot \nabla](\mathbf{u}+\epsilon \mathbf{v})-(\mathbf{u} \cdot \nabla) \mathbf{u}) d \Omega \\
& \lim _{\epsilon \rightarrow 0+} \frac{1}{\xi} \int_{\Omega}\left((\mathbf{u} \cdot \nabla) \mathbf{u}+(\xi \mathbf{v} \cdot \nabla) \mathbf{u}+(\mathbf{u} \cdot \nabla) \dot{\epsilon} \mathbf{v}+\left(\epsilon^{2} \cdot \vec{v} \cdot \vec{\nabla}-(\mathbf{u}-\nabla) \mathbf{u}\right) d \Omega\right. \\
& =\int_{\Omega}((\mathbf{v} \cdot \nabla) \mathbf{u}+(\mathbf{u} \cdot \nabla) \mathbf{v}) d \Omega \\
& L_{2}^{\prime}=-\lim _{\epsilon \rightarrow 0+} \frac{\mu}{\epsilon} \int_{\Omega}\left(\nabla^{2}(\mathbf{u}+\epsilon \mathbf{v})-\nabla^{2}(\mathbf{u})\right) d \Omega=-\int_{\Omega} \mu \nabla^{2} \mathbf{v} d \Omega \\
& L_{3}^{\prime}=-\lim _{\epsilon \rightarrow 0+} \frac{\mu}{\epsilon} \int_{\Omega}(\nabla(p+\epsilon q)-\nabla p) d \Omega=\int_{\Omega} \nabla q d \Omega \\
& L_{4}^{\prime}=\lim _{\epsilon \rightarrow 0+} \frac{1}{\epsilon} \int_{\Omega}(\alpha(\boldsymbol{u}+\xi \mathbf{v})-\alpha \mathbf{u}) d \Omega=\int_{\Omega} \alpha \mathbf{v} d \Omega \\
& L_{5}^{\prime}=\lim _{\epsilon \rightarrow 0+} \frac{1}{2 \downarrow} \int_{\partial \Omega} \nabla \cdot(\mathbf{u}+\epsilon \mathbf{v})-\nabla \cdot(\mathbf{u}) d \Omega=\int_{\partial \Omega} \nabla \cdot \mathbf{v} d \Omega
\end{aligned}
$$

It is possible to write the necessary condition for optimality $\left(L^{\prime}=0\right)$ in the weak form:

$$
\begin{array}{r}
L^{\prime}=\int_{\Omega}(2 \alpha \mathbf{u} \cdot \mathbf{v}+\mu \nabla \mathbf{u}: \nabla \mathbf{v}) d \Omega-\int_{\Omega}(\lambda \cdot(\mathbf{v} \cdot \nabla) \mathbf{u}+\lambda \cdot(\mathbf{u} \cdot \nabla) \mathbf{v}+ \\
\left.-\mu \lambda \cdot \nabla^{2} \mathbf{v}+\lambda \cdot \nabla q+\alpha \lambda \cdot \mathbf{v}\right) d \Omega-\int_{\Omega} \beta \nabla \cdot \mathbf{v} d \Omega-\int_{\partial \Omega_{\text {inlet }}} \gamma \cdot \mathbf{v} d \Omega-\int_{\partial \Omega_{\text {wall }}} \rho \cdot \mathbf{v} d \Omega
\end{array}
$$

If it is desired to implement Equation 3.32, it is wanted to eliminate the $\nabla^{2}$ by integration by parts in order to make the problem well-posed. This way:

$$
\begin{aligned}
L^{\prime}=\int_{\Omega}(\alpha \mathbf{u} \cdot \mathbf{v}+\mu \nabla \mathbf{u}: \nabla \mathbf{v}) d \Omega & -\int_{\Omega}(\lambda \cdot(\mathbf{v} \cdot \nabla) \mathbf{u}+\lambda \cdot(\mathbf{u} \cdot \nabla) \mathbf{v}+ \\
+\mu \nabla \lambda: \nabla \mathbf{v}+\lambda \cdot \nabla q+\alpha \lambda \cdot \mathbf{v}) d \Omega & -\int_{\Omega} \beta \nabla \cdot \mathbf{v} d \Omega+\int_{\partial \Omega} \mu \lambda \cdot \nabla \mathbf{v} \cdot n d S \\
& -\int_{\partial \Omega_{\text {inlet }}} \gamma \cdot \mathbf{v} d \Omega-\int_{\partial \Omega_{\text {wall }}} \rho \cdot \mathbf{v} d \Omega
\end{aligned}
$$

Converting Equation 3.33 to the strong form of the adjoint system, integrating by parts, it is possible to obtain Equation 3.37 since the equation is true for all $\mathbf{v}$ and $q$ in $\Omega$ : 


$$
\begin{array}{rc}
-(\nabla \mathbf{u}) \lambda+(\nabla \lambda)^{T} \mathbf{u}-\mu \nabla^{2} \lambda+\nabla \beta-\alpha \lambda=\frac{\partial J}{\partial \alpha} & \text { in } \Omega \\
\nabla \cdot \lambda=0 & \text { in } \Omega \\
\gamma=0 & \text { on } \partial \Omega_{\text {inlet }} \\
\rho=0 & \text { on } \partial \Omega_{\text {wall }}
\end{array}
$$

with $\partial \Omega=\partial \Omega_{\text {inlet }}+\partial \Omega_{\text {wall }}$

The adjoint problem now can be solved in the same way the forward problem due to its similarity in form. With the adjoint variables in hand the sensitivity of the problem, which is essential to the work, can now be determined by Equation 3.38.

$$
L=\frac{\partial J}{\partial \alpha}-\lambda \frac{\partial \mathbf{F}}{\partial \alpha}
$$

The sensitivity of of the complete optimization problem can be divided in diodicity, vorticity and structural parts. The following analysis shows the calculation for diodicity and vorticity, while the structural term due to its simplicity is calculated direct from the library Dolfin-Adjoint[FUNKE, 2013] that uses an automatic differentiator.

The optimization problem of diodicity can be divided in two problems as shown in Equation 3.39 according to energy dissipation.

$$
\min _{\alpha} J_{\Phi}=\frac{\Phi\left(\mathbf{u}_{f}, p_{f}\right)}{\Phi\left(\mathbf{u}_{r}, p_{r}\right)} \Rightarrow J^{\prime}=\frac{\Phi_{\left(\mathbf{u}_{f}, p_{f}\right)}^{\prime}}{\Phi\left(\mathbf{u}_{r}, p_{r}\right)}-\frac{\Phi\left(\mathbf{u}_{f}, p_{f}\right) \Phi_{\left(\mathbf{u}_{r}, p_{r}\right)}^{\prime}}{\Phi\left(\mathbf{u}_{r}, p_{r}\right)^{2}}
$$

Now the energy dissipation can be calculated using the Frechet derivative, since the derivative of the objective function is related to energy dissipation (see Equation 3.42).

$$
\begin{gathered}
\frac{\partial J_{\Phi}}{\partial \alpha}=\lim _{\epsilon \rightarrow 0+} \frac{J(\mathbf{u}+\epsilon \mathbf{v})-J(\mathbf{u})}{\epsilon}=\lim _{\epsilon \rightarrow 0+} \frac{1}{2 \epsilon} \int_{\Omega}(\alpha(\mathbf{u}+\epsilon \mathbf{v}) \cdot(\mathbf{u}+\epsilon \mathbf{v})-\alpha \mathbf{u} \cdot \mathbf{u} \\
+\mu \nabla(\mathbf{u}+\epsilon \mathbf{v}): \nabla(\mathbf{u}+\epsilon \mathbf{v})-\mu \nabla(\mathbf{u}):(\mathbf{u})) d \Omega \\
\frac{\partial J_{\Phi}}{\partial \alpha}=\lim _{\epsilon \rightarrow 0+} \frac{1}{2 \hbar} \int_{\Omega}\left(\alpha\left(\mathbf{u}-\mathbf{u}+2 \epsilon \mathbf{v} \cdot \mathbf{u}+\epsilon^{\natural} \mathbf{v}\right)-\alpha \mathbf{u} \cdot \mathbf{u}\right. \\
\mu\left(\nabla \mathbf{u}: \nabla \mathbf{u}+2 \hbar \nabla \mathbf{v} \cdot \nabla \mathbf{u}+\underline{\left.\epsilon^{2} \nabla \mathbf{v} \cdot \nabla \mathbf{v}\right)-}-\underline{\mu \mathbf{u}: \nabla \mathbf{u}}\right) d \Omega
\end{gathered}
$$




$$
\frac{\partial J_{\Phi}}{\partial \alpha}=\int_{\Omega}(2 \alpha \mathbf{u} \cdot \mathbf{v}+\mu \nabla \mathbf{u}: \nabla \mathbf{v}) d \Omega
$$

The vorticity optimization problem can be described in Equation 3.43.

$$
\begin{gathered}
\min \\
\alpha
\end{gathered} \quad J_{V o r}=-\operatorname{Vor}(\mathbf{u}, p)=-\int_{\Omega}(\nabla \times \mathbf{u}):(\nabla \times \mathbf{u}) d \Omega
$$

Now using the Frechet derivative, it is possible to determine the gradient of vorticity term (see Equation 3.45).

$$
\begin{gathered}
\frac{\partial J_{V o r}}{\partial \alpha}=\lim _{\epsilon \rightarrow 0+} \frac{J(\mathbf{u}+\epsilon \mathbf{v})-J(\mathbf{u})}{\epsilon}=\lim _{\epsilon \rightarrow 0+} \frac{1}{\epsilon} \int_{\Omega} \nabla \times(\mathbf{u}+\epsilon \mathbf{v}): \nabla \times(\mathbf{u}+\epsilon \mathbf{v}) d \Omega \\
\frac{\partial J_{V o r}}{\partial \alpha}=\int_{\Omega}-2(\nabla \times \mathbf{u}):(\nabla \times \mathbf{v}) d \Omega
\end{gathered}
$$

Finally, the gradient of diodicity and vorticity can be added to the output of the DolfinAdjoint, multiplying each term with each weight parameter $W_{F}, W_{V}$ and $W_{E}$, respectively, and, this way, it can be determined the sensitivity of the complete optimization problem (Equation 3.14). All this implementation is checked by finite difference and it can be found in Appendix C. 


\section{NUMERICAL IMPLEMENTATION}

\subsection{Programming environment}

All the implementation is written in Python with the help of FEniCS, which is a high level open-source $\mathrm{C}++$ and Python framework that solves partial differential equations, offering powerful capabilities, specially when parallel simulations are needed.

The Fenics library is a collection open source software components with the common goal to enable automated solution of differential equations. The components provide scientific computing tools for working with computational meshes, finite element variational formulations of ordinary and partial differential equations, and numerical linear algebra.

In order to provide a simple and consistent user interface, FEniCS wraps the functionality of other components and external software, and handles the communication between these components. Figure 33 shows an overview of the relationships between the components of FEniCS and external software. The main library called DOLFIN holds functions as both user interface and core component of FEniCS. All communication between an user program, other core components of FEniCS and external software is routed through wrapper layers that are implemented as part of the DOLFIN user interface. Variational forms expressed in the UFL form language are passed to the form compiler FFC to generate UFC code, which can then be used by DOLFIN to assemble linear systems. Then, this code generation depends on the finite element backend FIAT and the just-in-time compilation utility Instant that can be optimized by the FErari backend. Finally, the plotting capabilities provided by DOLFIN are implemented by Viper. Some of this communication is exposed to users of the DOLFIN $\mathrm{C}++$ interface, which requires an user to explicitly generate UFC code from a UFL form file by calling a form compiler on the command-line. DOLFIN also relies on external software for important functionality such as the linear algebra libraries PETSc, Trilinos, uBLAS and MTL4, and the mesh partitioning libraries ParMETIS and SCOTCH. 


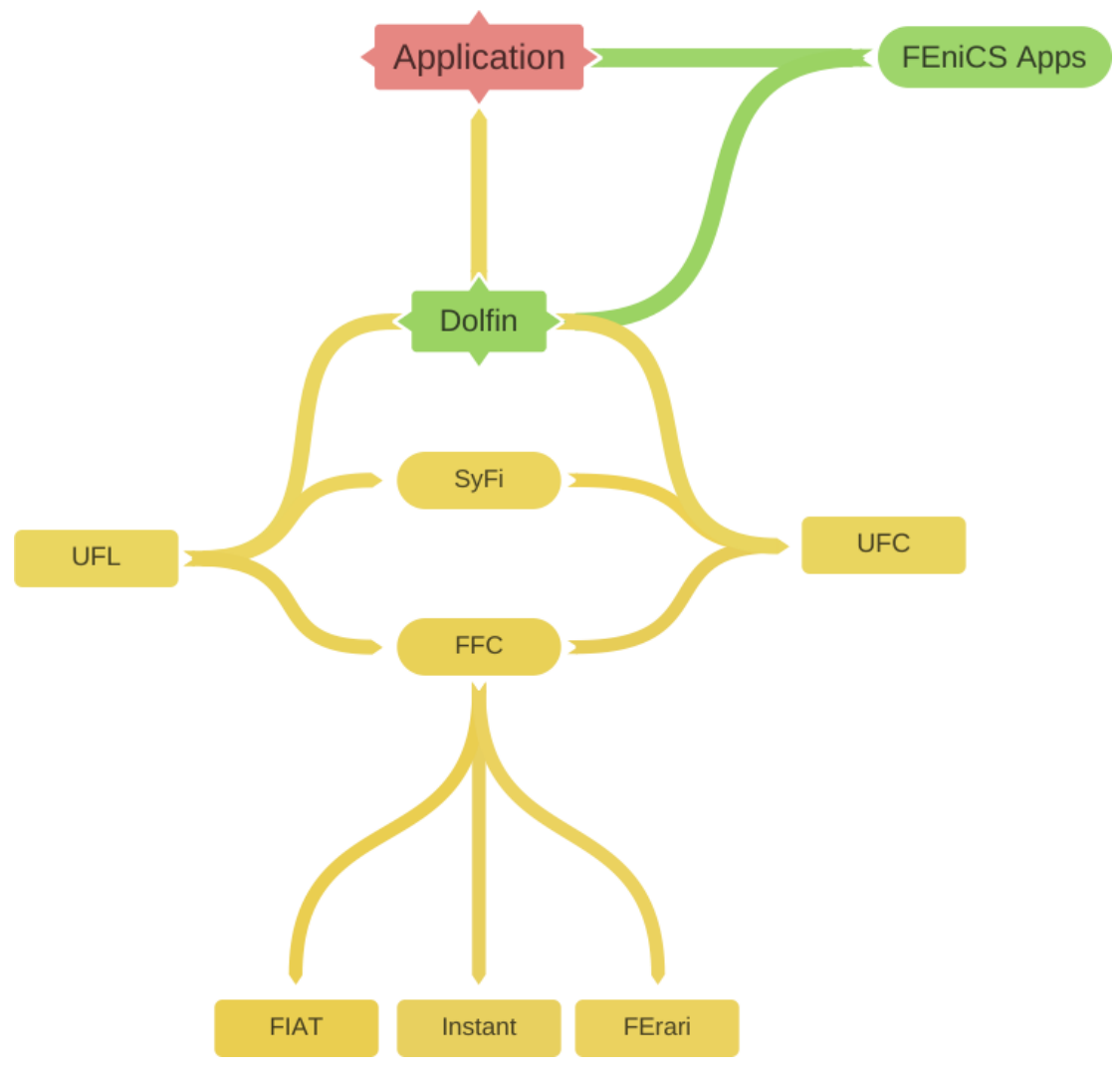

Figure 33: Overview of Fenics application.

For the optimization part, the minimization of compliance is easily calculated by another library called Dolfin-Adjoint. This tool can automatically calculate the sensitivity of many problems without additional implementation and that is the reason it is used in the structural simulation.

Figure 34 shows two approaches used in this work. For the fluid flow problem, the continuous adjoint equation is deduced and implemented in discrete form using Unified Form-Assembly Code (UFC) and the Fenics Form Compiler (FFC). In the structural analysis, the Dolfin-Adjoint can deduce the adjoint equations from the forward problem, using UFL and FFC and returning the necessary sensitivity values. The both values are combined in a class based Python program.

In order to check all the implementation exposed before, the sensitivity of the full problem is checked and can be found in Appendix C. 


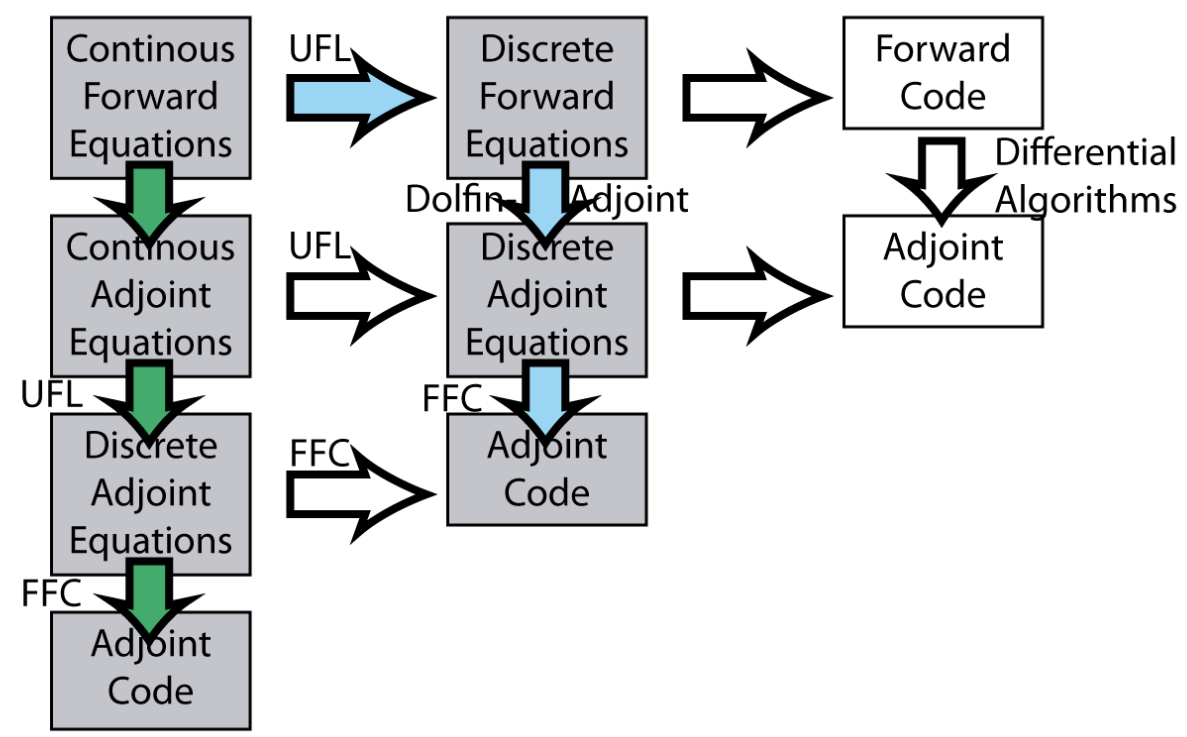

Figure 34: Programming scheme used in this work.

\subsection{Optimization procedure}

In the classic topology optimization, the initial values and boundary conditions must set first, then the finite element analysis gives the solution of the primal system. After this, the adjoint system is calculated to obtain the sensitivity of the problem. All these data are sent to the optimizer to obtain the new values of the design variables. The forward problem is recalculated and this whole process is repeated until a convergence criteria is achieved and the solution does not change significantly (see Figure 35).

The TOBS method[NADA, c] requires linearization of the optimization and that is done using the Octave (open source alternative to Matlab). The third part is the optimization and it occurs with the help of $\mathrm{CPLEX}^{\circledR}$ optimizer (the most efficient algorithm for binary variables found). This process run all over until a convergence criteria is achieved. All the process is illustrated in Figure 35.

In problem with solid-fluid interaction, the full optimization problem (Equation 3.11) requires more effort, thus the dark blue box in Figure 35 is actually more complex. This is represented in Figure 36, where both forward and reverse flow are simulated to obtain the primal solution of $\{\mathbf{u}, p\}$ to then simulate the structural analysis and, thus, the objective function with all terms can be calculated. After this, the adjoint system is simulated to obtain the adjoint variable values of flow analysis to calculate the sensitivity. This is added in the end by the derivative calculated by Dolfin-Adjoint of structural analysis, and 


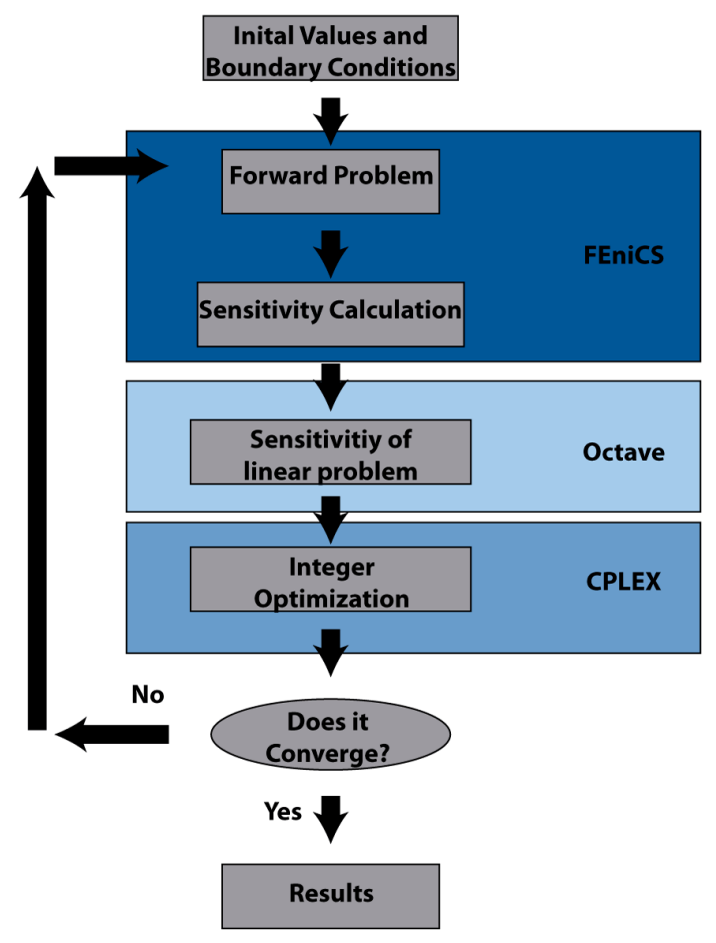

Figure 35: Flowchart of the Topology Optimization procedure with the TOBS Method.

then the correct gradient of the problem is determined.

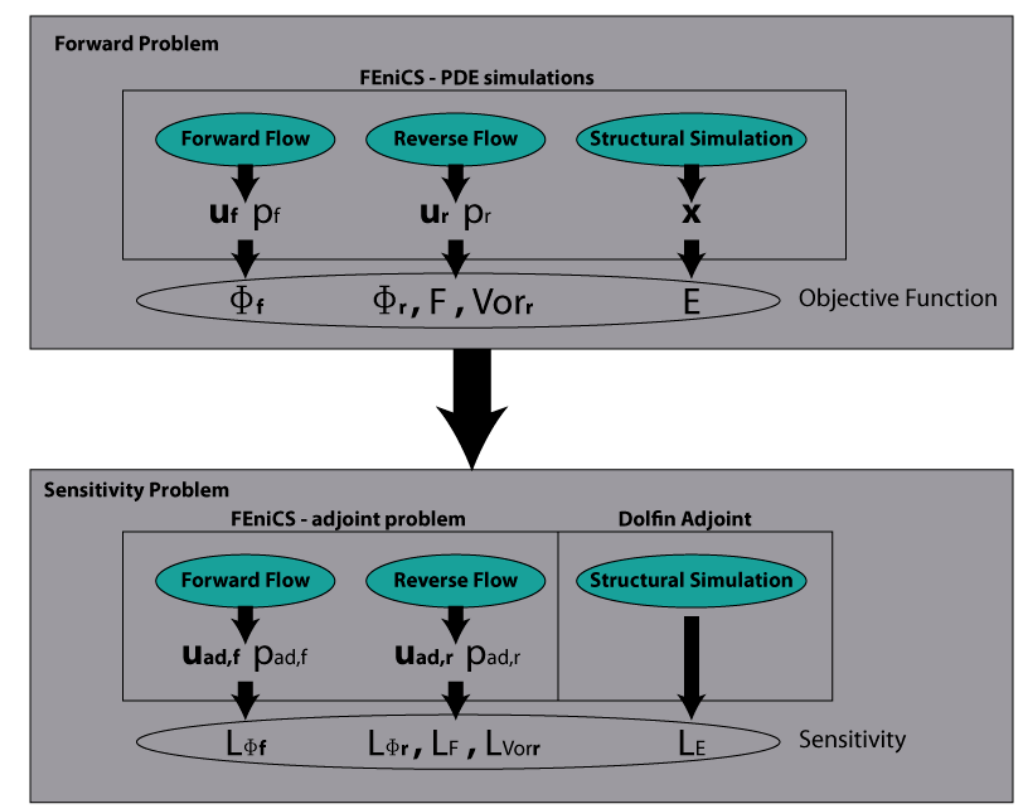

Figure 36: Detailed implementation of both forward and adjoint problem of Fenics block in Figure 35. 


\section{RESULTS}

In this chapter the results are presented, starting with the validation of the TOBS method in Fluid, followed by analysis of fluid diodes. Then, an approach of designing fluid diodes and labyrinth seals is developed.

The results presented in the following sections are computed with an Intel(R) Core(TM) i7-6700K CPU (4.00GHz) machine with an Arch Linux distribution and $24 \mathrm{~Gb}$ of RAM memory.

\subsection{Fluid topology optimization with TOBS}

The first contribution of this work is to implement TOBS method into the fluid flow optimization. Thus, the purpose of this section is to validate the method with classical problems such as double pipe stokes problem [BORRVALL; PETERSSON, 2003] and Navier-Stokes varying Reynolds $(R e)$ number.

\subsubsection{Classical Stokes problem}

In this section the classical Stokes problem is presented as illustrated in Figure 37, which represents two inlets at the left border of the domain $\Omega$ and two outlets on the right. The considered objective function is the minimization of the energy dissipation. The volume constraint of $1 / 3$ of domain occupied by fluid is employed.

The boundary conditions used in this section has a parabolic velocity profile with maximum value of $1[\mathrm{~m} / \mathrm{s}]$ (which corresponds to a Reynolds Number $R e=0.1$ defined in Equation 5.1 used for all numerical examples) on both inlets and outlets, and walls with no-slip condition. These conditions are illustrated in Figure 37.

$$
R e=\frac{\rho u_{a v} D}{\mu}=u_{a v} D
$$


where $\rho$ is fluid density, $u_{a v}$ is magnitude of average inlet velocity, $D$ is inlet length, and $\mu$ is dynamic viscosity of fluid.

The TOBS parameters used are $\varepsilon=0.1$ and flip limits $\beta=0.2$. The employed mesh in this example is shown in Figure 38. The result considering $\delta=1.5 m$ [BORRVALL; PETERSSON, 2003] is illustrated in Figure 39.

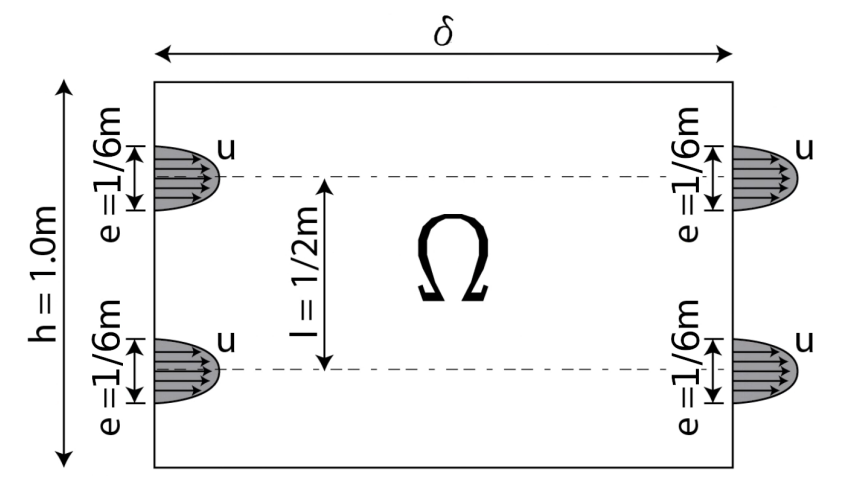

Figure 37: Design domain for the classic Stokes problem with $\mathbf{u}$ according to $R e$ value (spatial dimensions in $\mathrm{m}$ ).

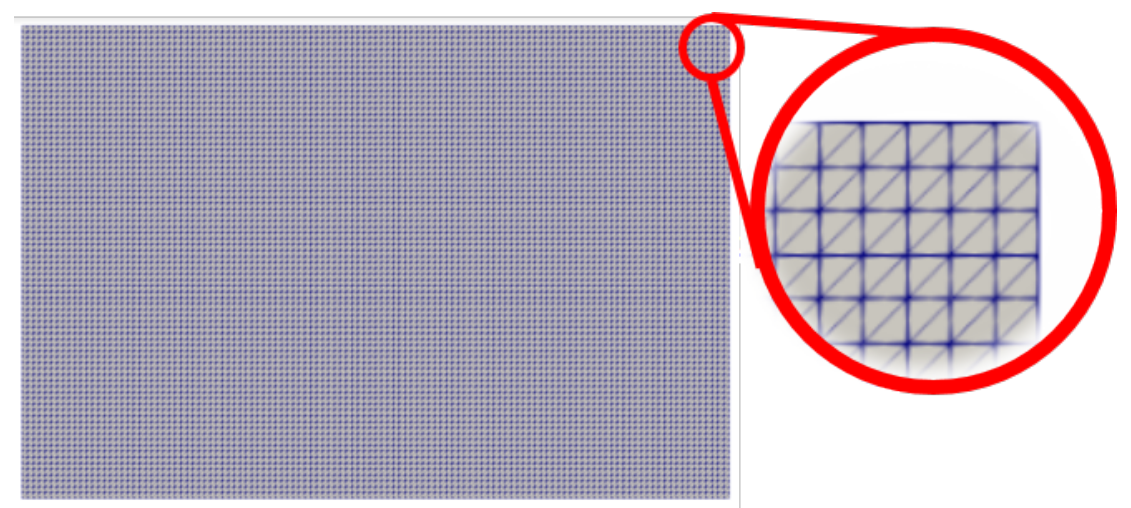

Figure 38: Mesh of the Stokes Problem with regular 9600 elements.

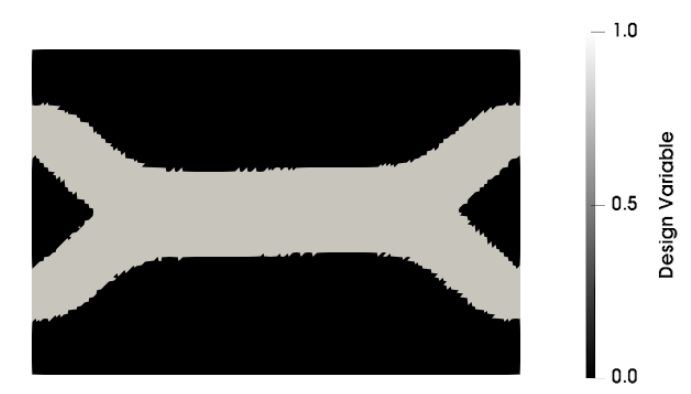

Figure 39: Topology optimization Stokes results for $\delta=1.5$. 
The convergence plot of the objective function for this case is presented in Figure 40 and the volume constraint value. The increase of the objective function in the initial iterations can be addressed to the volume constraint not being satisfied. After the constraint value is achieved, the optimization proceeds normally with decrease of the objective function value.

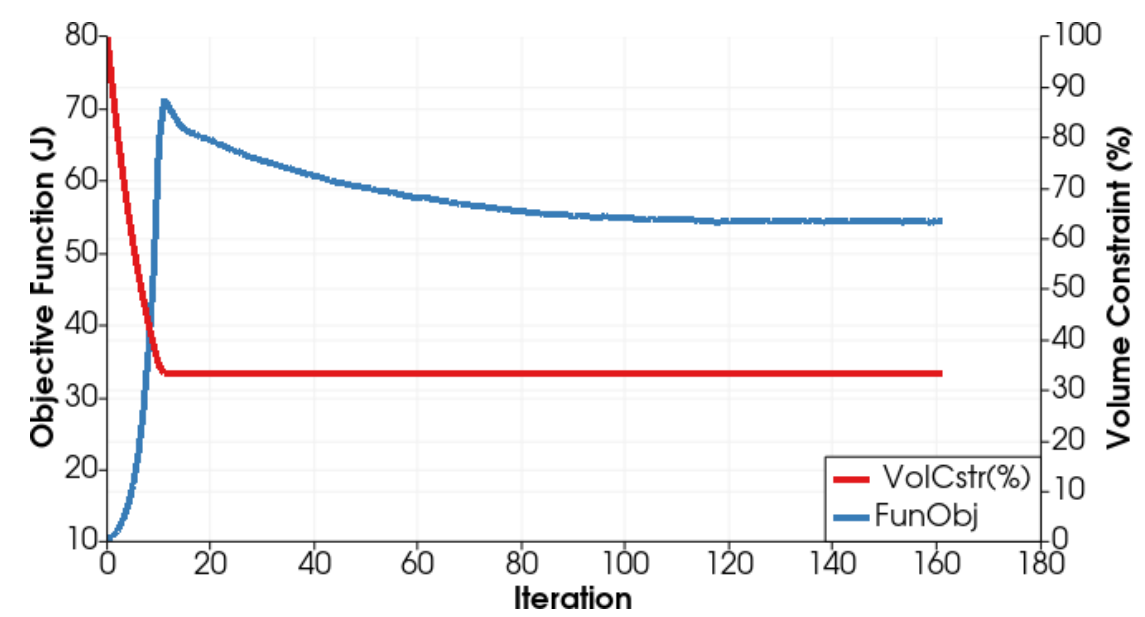

Figure 40: Objective function convergence for Stokes classic problem $(\delta=1.5)$.

The evolution of the optimization is shown in Figure 41, in which can be seen that its interface with a clear contour and intermediate values are absent (no grayscale). The comparison of results with proposed and traditional method [BORRVALL; PETERSSON, 2003] can be seen in Figure 42.

Figure 43 presents iteration number 10 of the double pipe problem on both traditional and TOBS methods. The large gray area makes hard to determine distance to the wall and this can be problematic in more complex problems where the wall position is important. 


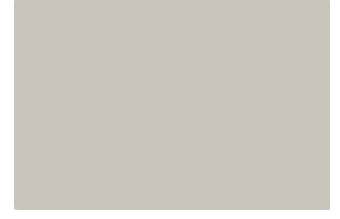

(a) Iteration number: 0

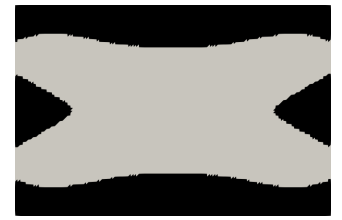

(d) Iteration number: 5

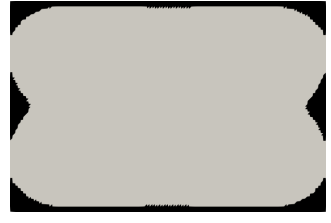

(b) Iteration number: 1

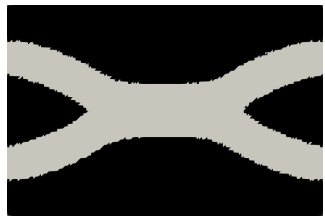

(e) Iteration number: 30

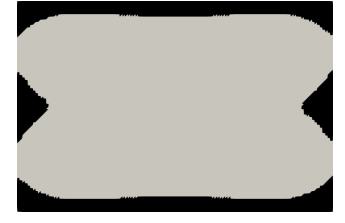

(c) Iteration number: 2

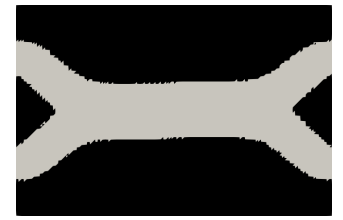

(f) Iteration number: 160

Figure 41: Topology optimization results evolution during optimization.

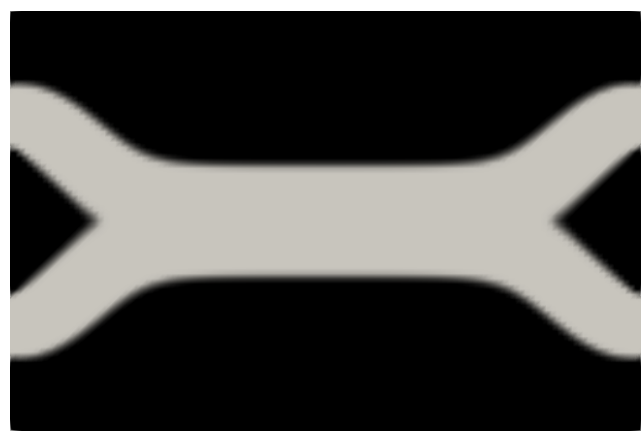

(a) Traditional method.

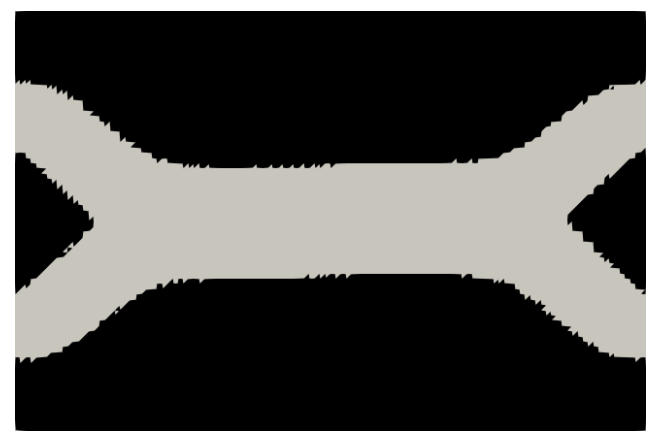

(b) TOBS method.

Figure 42: Comparison between methods.

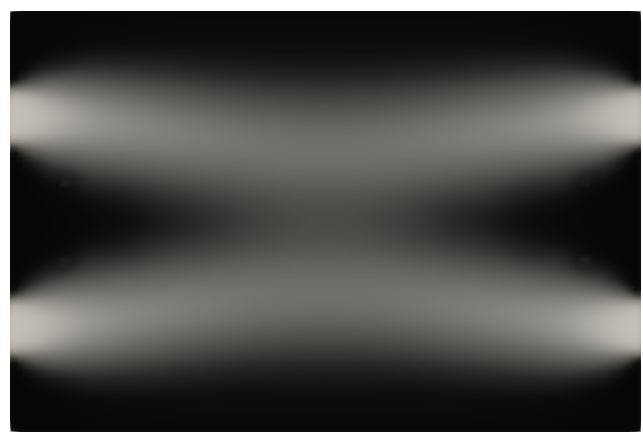

(a) Traditional method in iteration 10 .

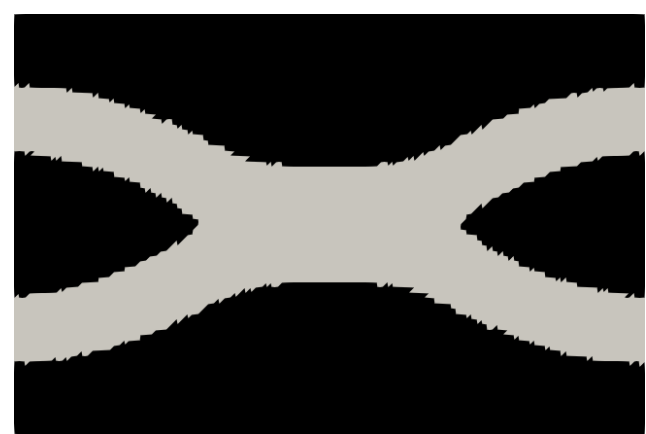

(b) TOBS method in iteration 10 .

Figure 43: Comparison between methods during optimization process.

Table 2 shows the comparison of the achieved objective function from literature [CHALLIS; GUEST, 2009], with objective function $G(\mathbf{u}))$ defined in Equation 5.2. 


$$
G(\boldsymbol{u}, \boldsymbol{\alpha})=\frac{\mu}{2} \int_{\Omega}\|\nabla \boldsymbol{u}\|^{2} d \Omega
$$

Table 2: Functional value $G$ comparison [CHALLIS; GUEST, 2009] for resulting topologies of double channel problem (mesh: 135000 elements $(\delta=1.5)$ and 14400 elements with $\delta=1.0 \mathrm{~m}$ and $\left.\kappa_{\max }=2.5 \cdot 10^{6}\right)$

\begin{tabular}{ccc}
\hline Case & $\delta=1.0 m$ & $\delta=1.5 m$ \\
\hline Analytical & 32.00 & - \\
Proposed method & 31.28 & 32.92 \\
Challis \& Guest & 31.68 & 32.86 \\
Borrvall \& Petersson & 25.67 & 27.64 \\
\hline
\end{tabular}

With $\delta=1.5 m$, due to the jagged solution (see Figure 44) that brings the proposed method, it indicates that smoothing methods may be necessary depending on the case study.

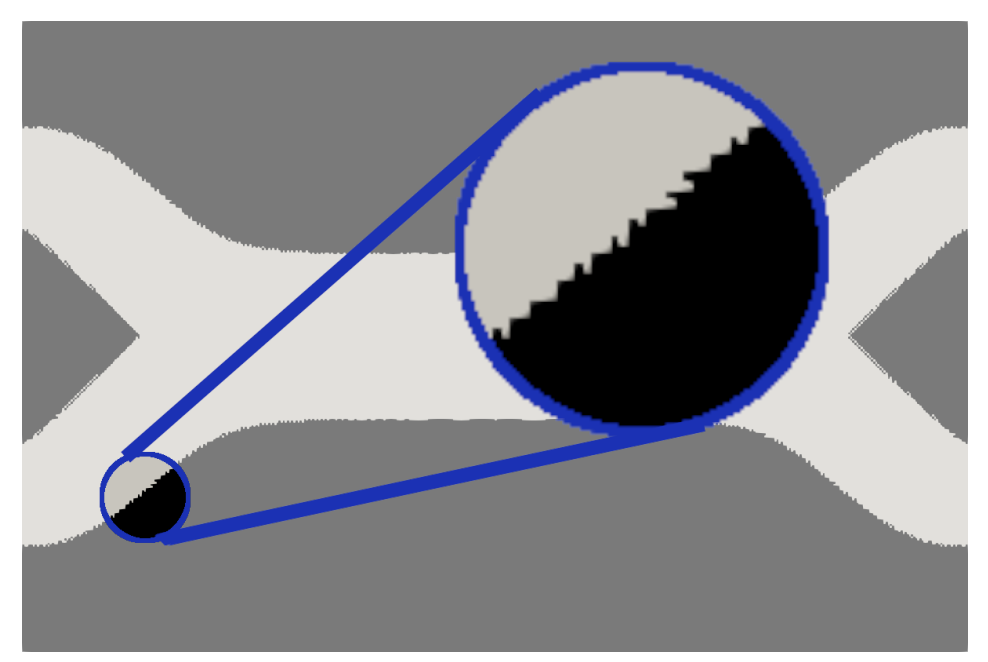

Figure 44: Jagged contour caused by the proposed method in some case studies.

\subsubsection{Mesh independence}

This subsection studies the behavior of the TOBS in the classical Stokes problem with a mesh independence test. The meshes used are described in Table 3, with N1 being the coarsest mesh and N3 the finest. The final topology of each mesh is shown in Fig 45. 
Table 3: Mesh size used in mesh independence test

\begin{tabular}{cc}
\hline$n$ & mesh elements \\
\hline N1 & 15000 \\
N2 & 60000 \\
N3 & 135000 \\
\hline
\end{tabular}

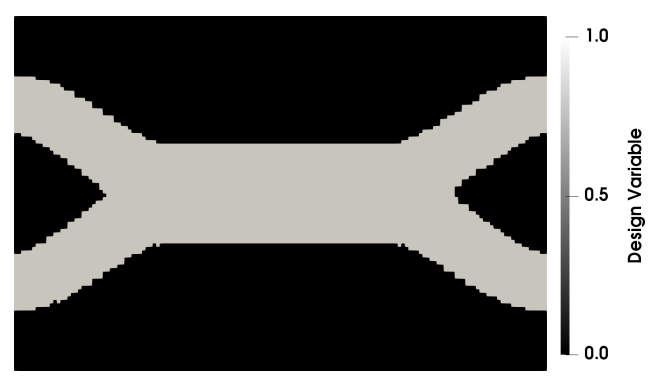

(a) Mesh N1

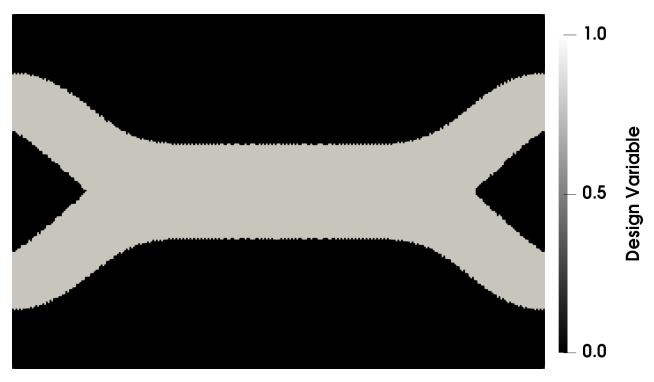

(b) Mesh N2

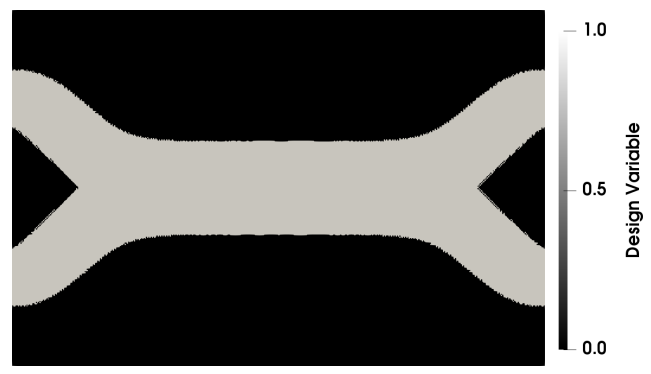

(c) Mesh N3

Figure 45: Results of the mesh independence test.

The increase in the number of elements has not changed the local minimum, which indicates that the optimization can be performed in coarser meshes when optimizing the Stokes model.

The convergence history of the objective function value for the mesh independence test can be visualized in Fig. 46. The finer mesh presented the lower objective function, although all the meshes tested reached the same local minimum. 


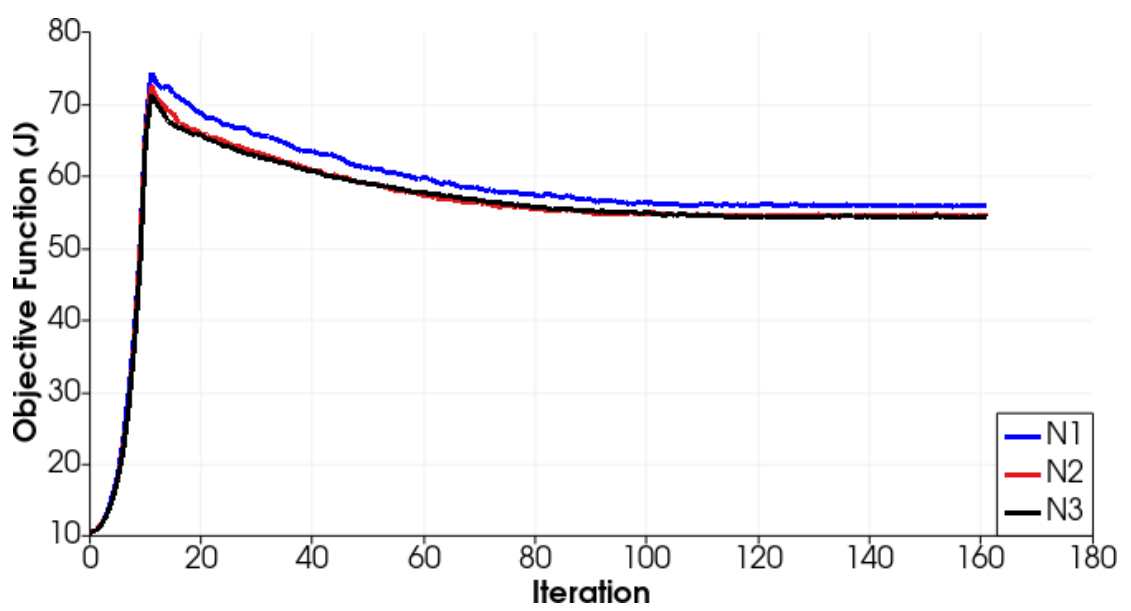

Figure 46: Objective function convergence of the mesh independence test.

Besides the absence of filter in these examples, another difference of the implementation in this work and the implementation performed by [NADA, c, d] is the absence of a stabilization method between successive optimization iterations.

\subsubsection{Influence of $\kappa_{\max }$ parameter}

This subsection studies the influence of the $\kappa_{\max }$ (described in Section 3.1) value with a domain with shorter length $(\delta=1)$.

When the $\delta$ value is set to 1.0, while maintaining the discretization $80 \mathrm{x} 80$ (Fig. 38), it is expected that the optimized topology is two straight lines, as shown in [BORRVALL; PETERSSON, 2003]. However, if no change in the $\kappa_{\max }$ value is made it is harder to achieve the corresponding minimum, which is two horizontal straight lines in the design domain. The results achieved with 160 iterations and with different $\kappa_{\max }$ values are shown in Figure 47. 


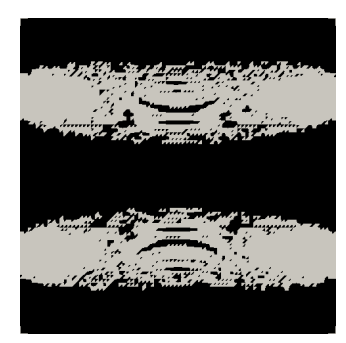

(a) $\kappa_{\max }=2.5 \cdot 10^{2}$

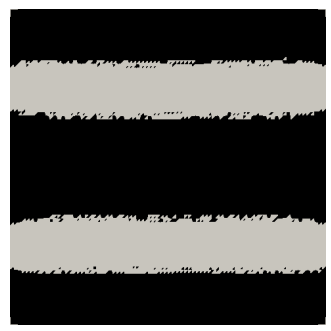

(b) $\kappa_{\max }=2.5 \cdot 10^{3}$

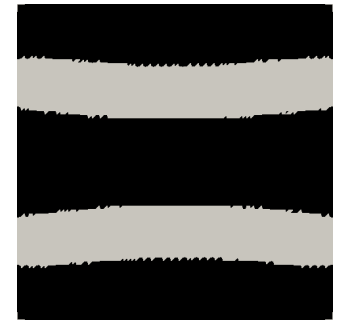

(c) $\kappa_{\max }=2.5 \cdot 10^{4}$

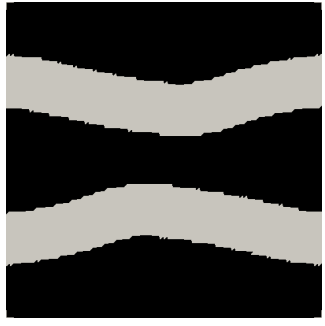

(d) $\kappa_{\max }=2.5 \cdot 10^{5}$

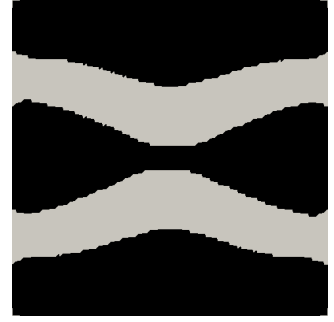

(e) $\kappa_{\max }=2.5 \cdot 10^{6}$

Figure 47: Results with different $\kappa_{\max }$ values at iteration 160.

Figure 47a-b shows an undesirable islands problem. They occur due to small values of $\kappa$, which implies fluid flow inside solid parts. This causes the islands because it makes the presence of fluid or solid less clear for the algorithm. In Figures $47(\mathrm{a})$ and $47(\mathrm{~b}), \kappa_{\max }$ has relatively small values, $2.5 \cdot 10^{2}$ and $2.5 \cdot 10^{3}$, respectively, bringing island in the design domain since fluid can flow through solid parts. As the $\kappa_{\max }$ increases (Figs. 47(c-e)), this problem disappears, however, the straight-channels minimum is more difficult to be reached. This indicates that a continuation in the $\kappa_{\max }$ value should be performed as the optimization proceeds. Thus, starting the algorithm with $\kappa_{\max }=2.5 \cdot 10^{1}$ and multiplying its value by 10 every 10 iterations until it reaches $\kappa_{\max }=2.5 \cdot 10^{4}$, the straight-channel topology is achieved, as shown in Figure 48. The impact of changing $\kappa_{\max }$ on the objective function value can be seen in Figure 49.

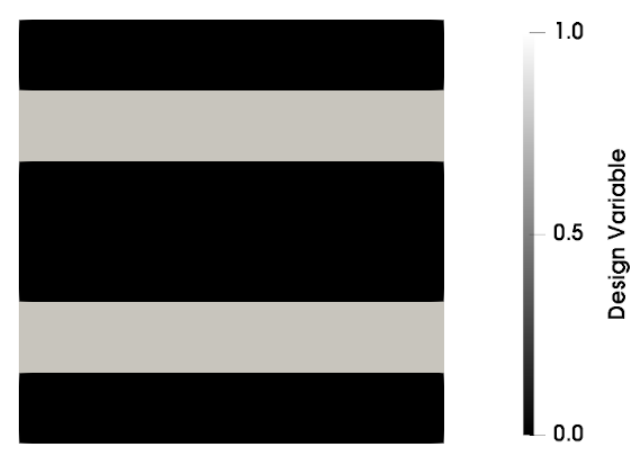

Figure 48: Topology optimization Stokes results for $\delta=1.0$. 


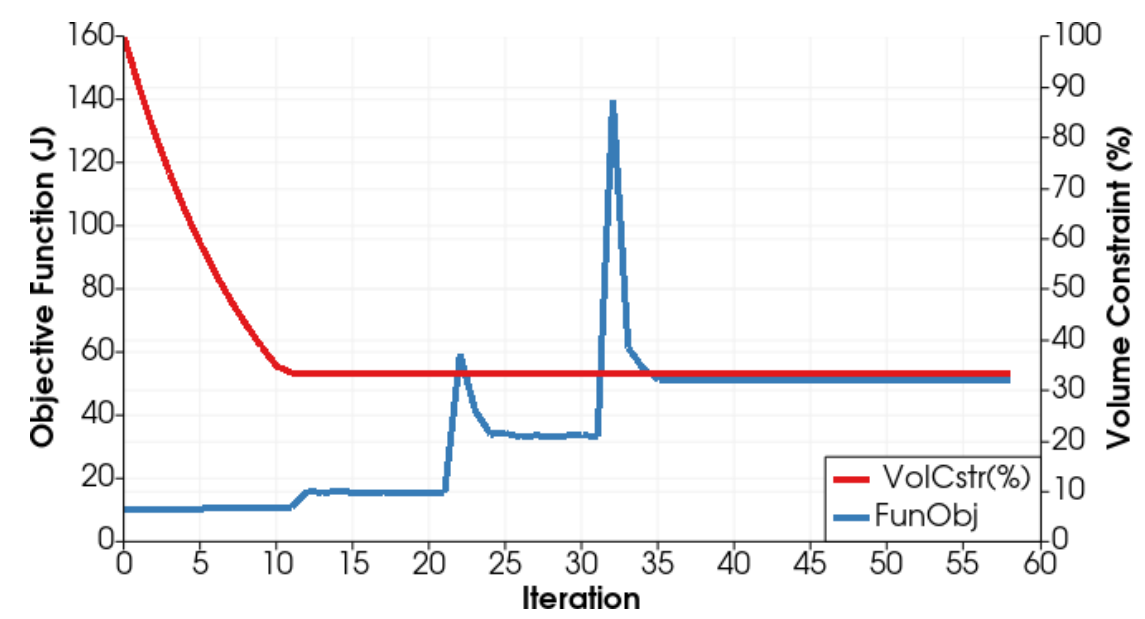

Figure 49: Objective function convergence for Stokes classic problem $(\delta=1.0)$ with continuation of $\kappa_{\max }$.

\subsubsection{Influence of the penalization term $(q)$}

The penalization term $q$ of the material model interpolation is very important in the traditional topology optimization in fluids, given that depending on its value a different topology can be reached. The literature indicates that in order to avoid a local minimum a continuation should be performed in the $q$ value [BORRVALL; PETERSSON, 2003]. However, with the methodology proposed in this work it can be set to 1.0 and no gray-scale appear.

A more complete analysis can be seen in Figure 50. A smaller value of $q$ brings the island problem on the topology contour. More over, when the value decreases to $q=0.01$ the solution is trapped into an undesirable local minimum (see Figure 50 (c)). 


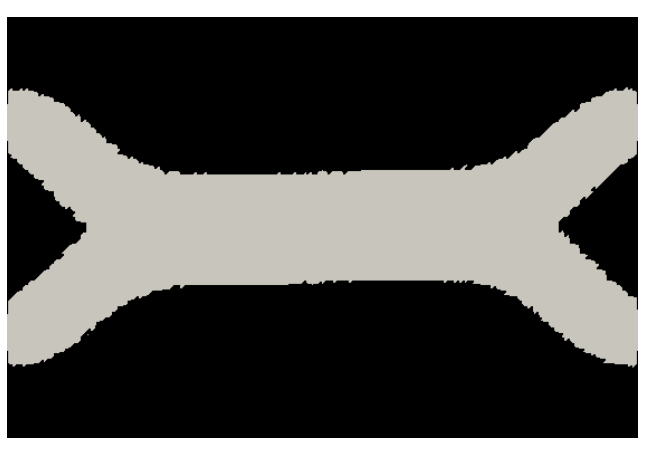

(a) $q=1.0$

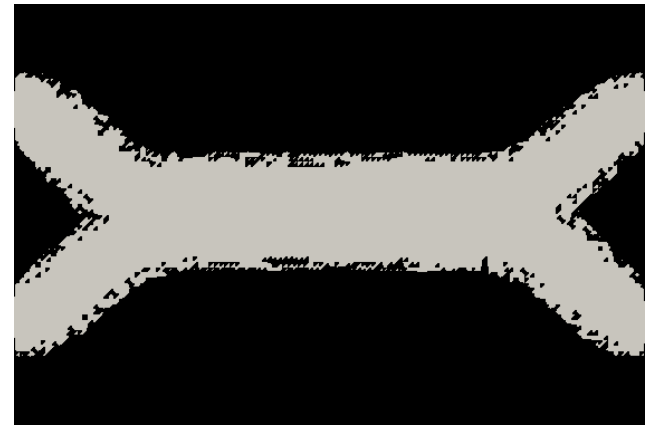

(b) $q=0.1$

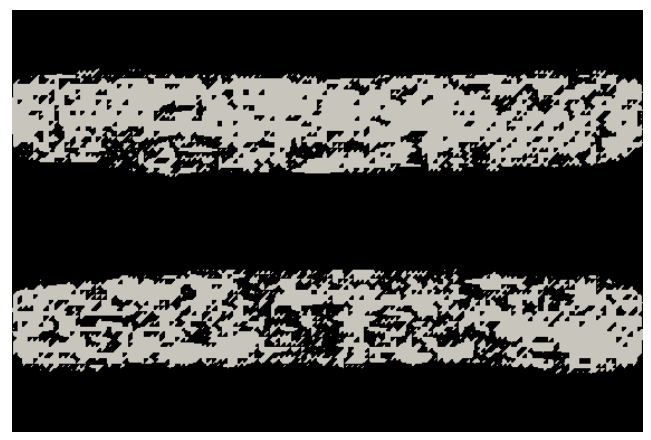

(c) $q=0.01$

Figure 50: Results of the influence of $q$ values in the binary optimization.

\subsubsection{Influence of $\varepsilon$ parameter}

All results in this work are presented for $\varepsilon=0.1$. Hence, in this section, aiming to evaluate the $\varepsilon$ influence, two cases are explored: $\varepsilon=0.15$ and $\varepsilon=0.01$. Both cases reach the expected minimum, however, the path each one has taken is relatively different, as it can be seen in Figure 51.

It can be seen in Figure 52 that the bigger the $\varepsilon$ value is, the sooner the optimization reaches the local minimum due to larger steps. When using large $\varepsilon$ values, it would result in a small overshoot effect in the objective function when the volume constraint is reached. 


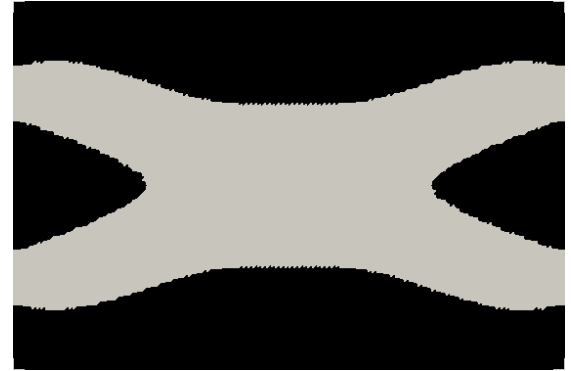

(a) Iteration number: 5 with $\varepsilon=0.15$

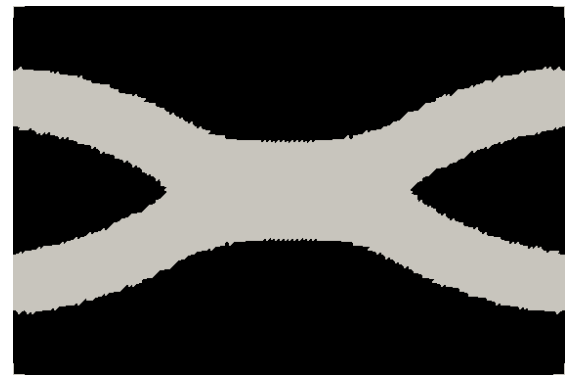

(c) Iteration number: 15 with $\varepsilon=$ 0.15

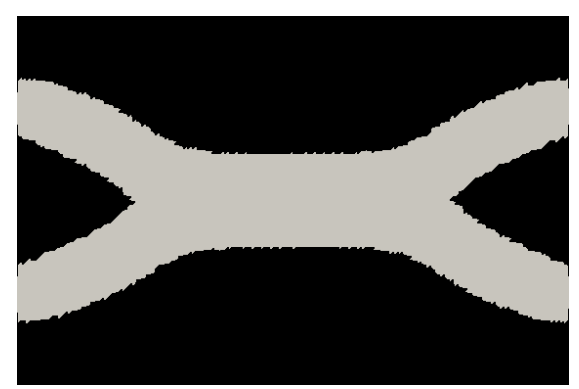

(e) Iteration number: 50 with $\varepsilon=$ 0.15

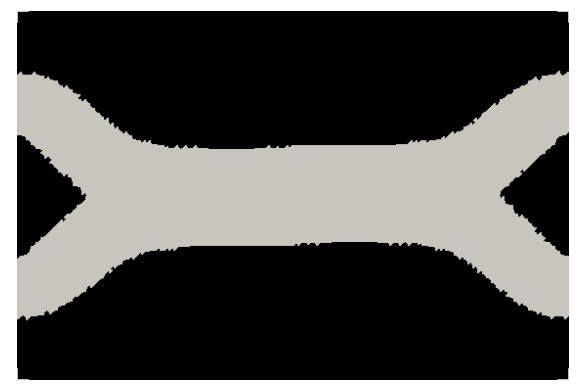

(g) Iteration number: 160 with $\varepsilon=$ 0.15

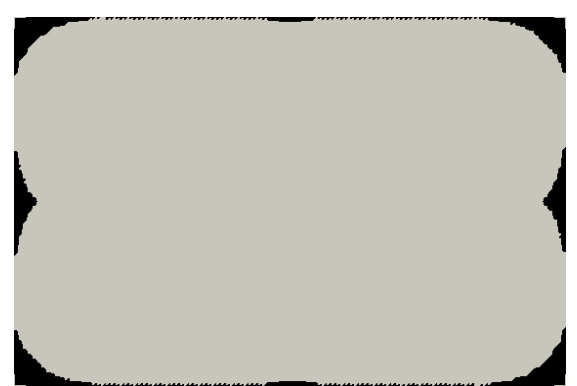

(b) Iteration number: 5 with $\varepsilon=0.01$

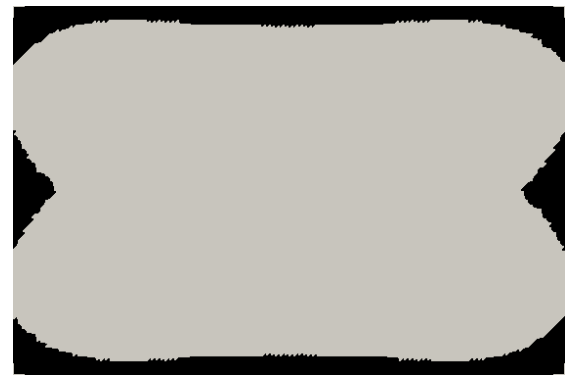

(d) Iteration number: 15 with $\varepsilon=$ 0.01

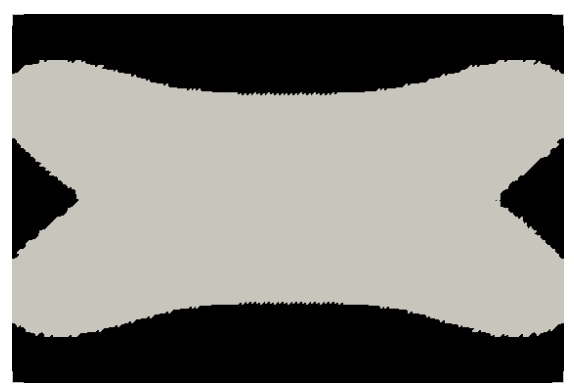

(f) Iteration number: 50 with $\varepsilon=$ 0.01

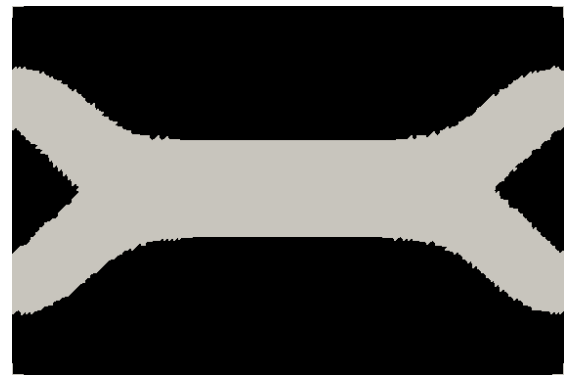

(h) Iteration number: 160 with $\varepsilon=$ 0.01

Figure 51: Results evolution during optimization for different $\varepsilon$ values. 


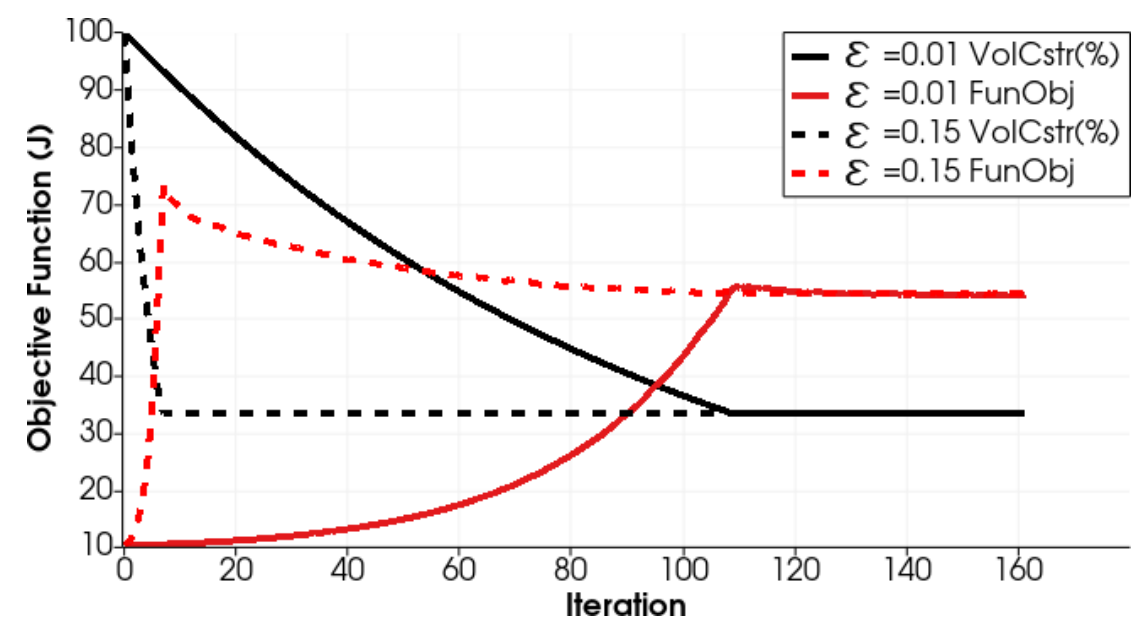

Figure 52: Objective function convergence curves for different $\varepsilon$ values.

\subsubsection{Influence of $\beta$ parameter}

The investigation on the truncation error constraint defined by the $\beta$ value is performed in this subsection. The use of TOBS methodology in structural problems usually present some challenges regarding, for example, structural members breaking during optimization and leading to drastic changes in the topology with a high number of elements flips [NADA, c]. The parameter $\beta$ restricts such changes. The present fluid problem does not face these challenges and, therefore, changing the $\beta$ value did not change the final solution. On the other hand, it is beneficial to keep $\beta$ in the optimization formulation as it keeps the truncation error small and as it may be important for other yet unexplored fluid problems.

From the results presented in this section, the authors advise to explore different values of the optimization parameters and initial guesses, in order to try to reach different solutions if the TOBS is intended to be used in other applications.

\subsubsection{Navier-Stokes problem}

Besides the first verification of the topology optimization implementation, it is important to check the behaviour of the optimization when the incompressible Navier-Stokes equations are considered. First, a low Reynolds number case is explored, then some cases with higher Reynolds numbers are analyzed.

Two bend pipe domains are defined: one shown in Figure 53(a) with the corresponding boundary conditions, and other shown Figure 53(b), which describes the same problem with a squared hole in the center. The corresponding meshes are shown in Figures 54(a) 
and 54(b), respectively.

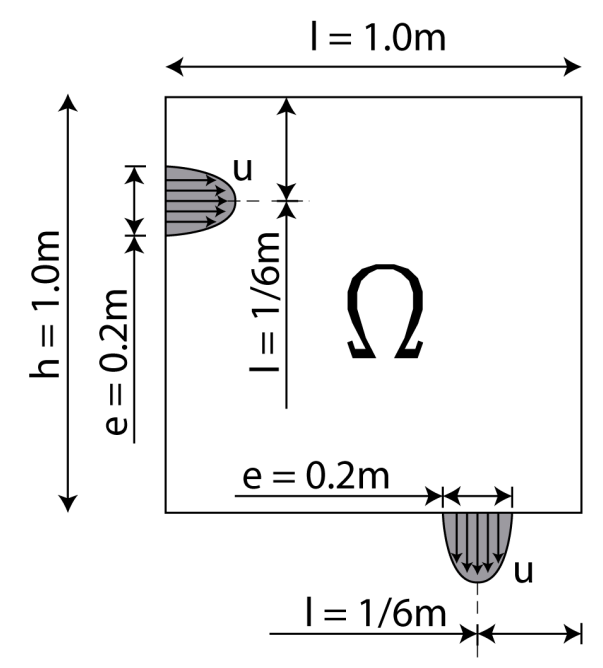

(a) Bending pipe domain.

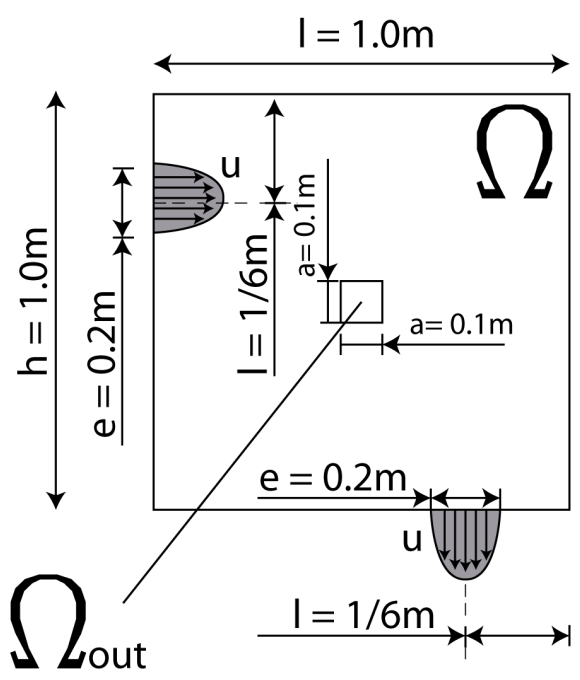

(b) Bending pipe domain with a hole.

Figure 53: Design domain for a Navier-Stokes problem with $\mathbf{u}$ according to $R e$ value (spatial dimensions in $\mathrm{m}$ ).

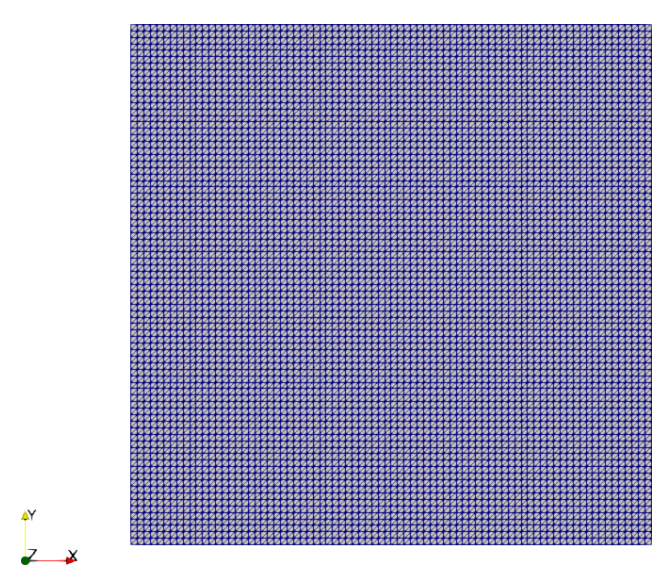

(a) Finite element mesh of bending pipe domain (25600 elements).

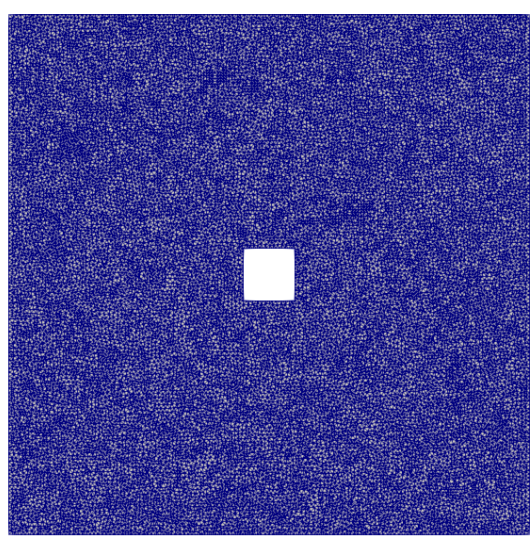

(b) Finite element mesh of bending pipe domain with a hole (22960 elements).

Figure 54: Finite element meshes for the Navier-Stokes problem.

\subsubsection{Low Reynolds number}

The first set of results are analyzed with low Reynolds Number $(\mathrm{Re}=1)$ with $\varepsilon=0.1$ and flip limits $\beta=0.2$ for this problem. A wide range of Reynolds number is analyzed for this problem in the subsection 5.1.2.2. 


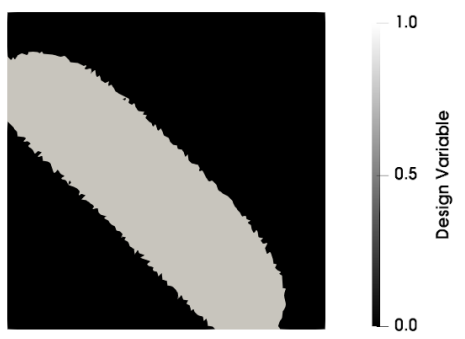

(a) Bending pipe domain.

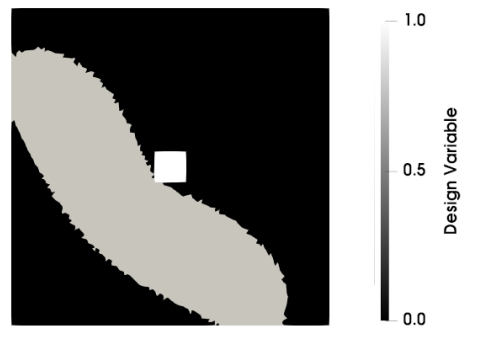

(b) Bending pipe domain with a hole.

Figure 55: Topology optimization for Navier-Stokes example.

The results of this optimization problem are presented in Figure 55 (a-b) and they are very similar to the literature [SÁ et al., 2016]. In order to illustrate the optimization process, Figure 56 shows how the $\{0,1\}$ optimization step goes by iteration in the more complex design domain. The convergence curve of the objective function is illustrated in Figure 57, which presents a similar behaviour as the convergence curve shown in Section 5.1.1.

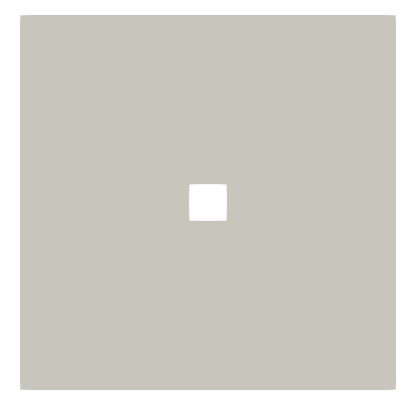

(a) Iteration number: 0

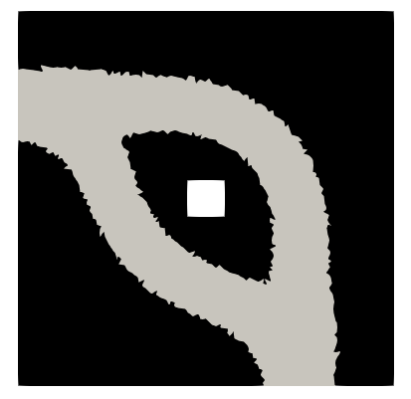

(d) Iteration number: 6

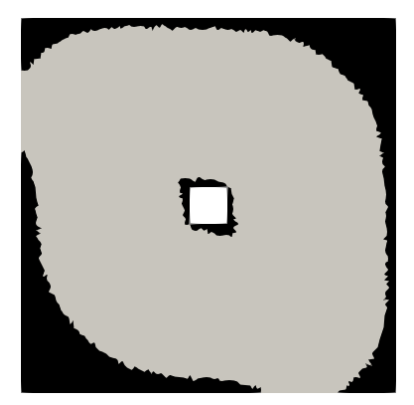

(b) Iteration number: 1

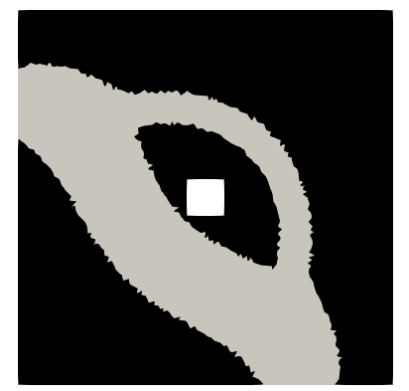

(e) Iteration number: 20

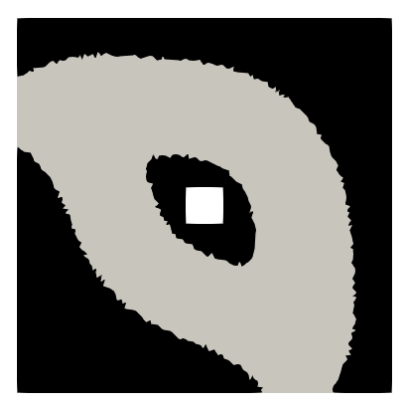

(c) Iteration number: 3

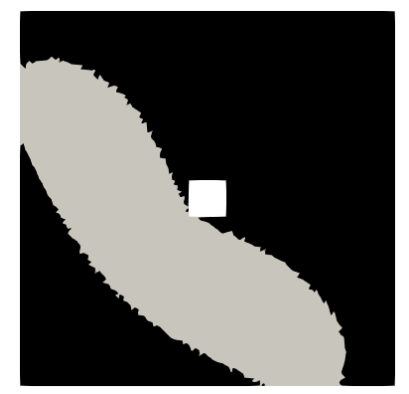

(f) Iteration number: 47

Figure 56: Results evolution during optimization. 


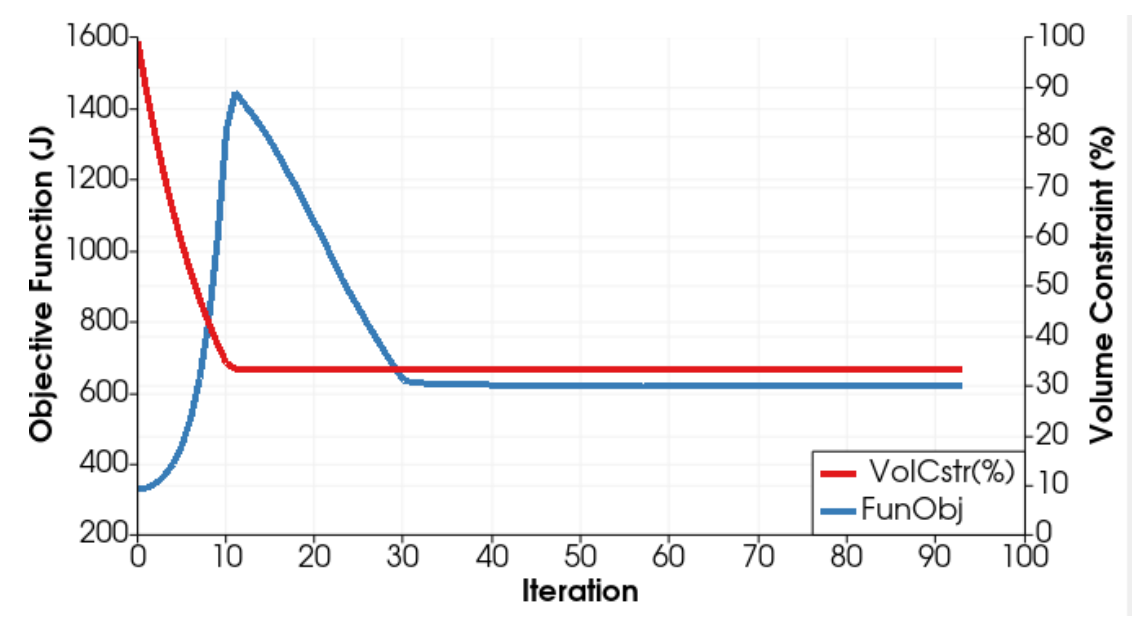

Figure 57: Objective function convergence for the Navier-Stokes problem considering the design domain with a central squared hole $(\delta=1.5)$.

The reason that the convergence curve (Fig. 57) rises strongly in the beginning and then falls is due to the volume constraint, since the initial domain is fluid full.

\subsubsection{Wide range of Reynolds numbers}

To explore the algorithm behavior under the Navier-Stokes modelling, other Reynolds numbers are chosen among $R e=\{1,5,10,50,100\}$, and results are presented in Figure 58. The results for the bend pipe without obstacle (Fig. 55(a)) are very similar for all cases. Thus, only results obtained for the domain with a squared hole in the center (Fig. $55(\mathrm{~b}))$ are shown, since it is easier to observe the differences among the final topologies. The results can be seen in Figure 58. 


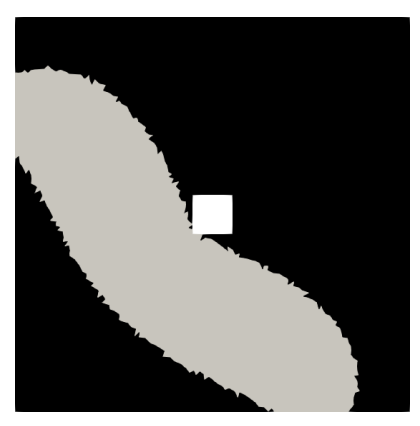

(a) Reynolds number: 1

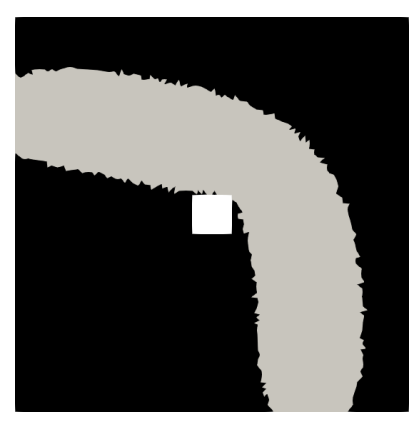

(b) Reynolds number: 5

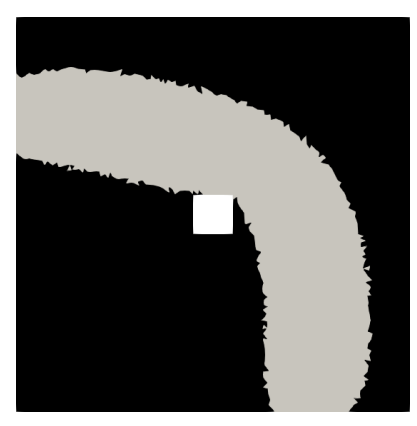

(c) Reynolds number: 10

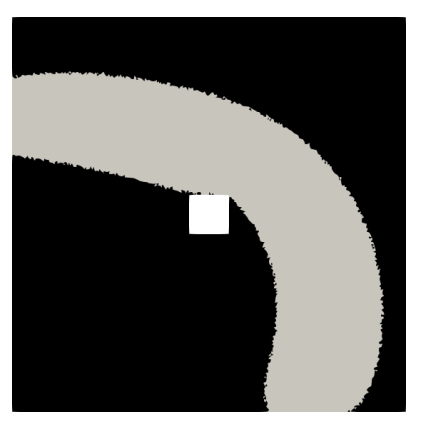

(d) Reynolds number: 50

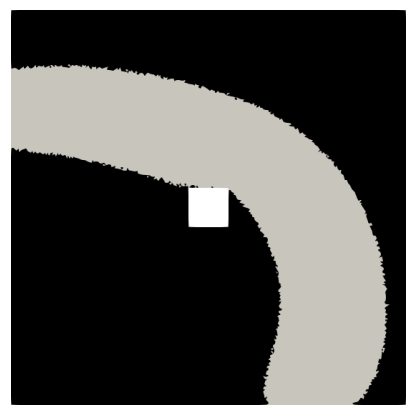

(e) Reynolds number: 100

Figure 58: Topology optimization results for different Re number.

As the Reynolds number increases, the algorithm tends to change the channel position, from the bottom of the obstacle to the top. The result from Figure 58(a) represents a very low Reynolds number and it could be achieved just by neglecting the convection term in the Navier-Stokes model, i.e., with the Stokes model.

Figures 58(b) and (c) present similar behaviours, with medium Reynolds number. The highest Reynolds number analyzed shows that the curvature intensifies with the velocity increase (Figures 58(d) and (e)). Higher inlet velocities than presented here, have not been analyzed here due to the complexity of solving Navier-Stokes for high Reynolds numbers which does not belong to the scope of this work.

\subsubsection{D fluid problem}

To show that the methodology can be easily expanded to 3D problems, the problem illustrated in Figure 59 is analyzed. The problem presents a tri-dimensional simulation of four channels, with boundary conditions considering parabolic velocity profile on both inlets and outlets, with $R e=1, \varepsilon=0.1 \beta=0.2$ and applying a volume constraint of $1 / 6$ of total volume. The dimensions are similar to the $2 \mathrm{D}$ previous problem, keeping the entrance with a diameter of $0.2 \mathrm{~m}$ and a parallelepiped with dimensions $1.0 \mathrm{~m} \times 1.0 \mathrm{~m} \times 1.5 \mathrm{~m}$. The regular discretization of the 3D problem is shown in Figure 60, which shows an 
unstructured mesh. The corresponding results of the topology optimization can be seen in Figure 61.

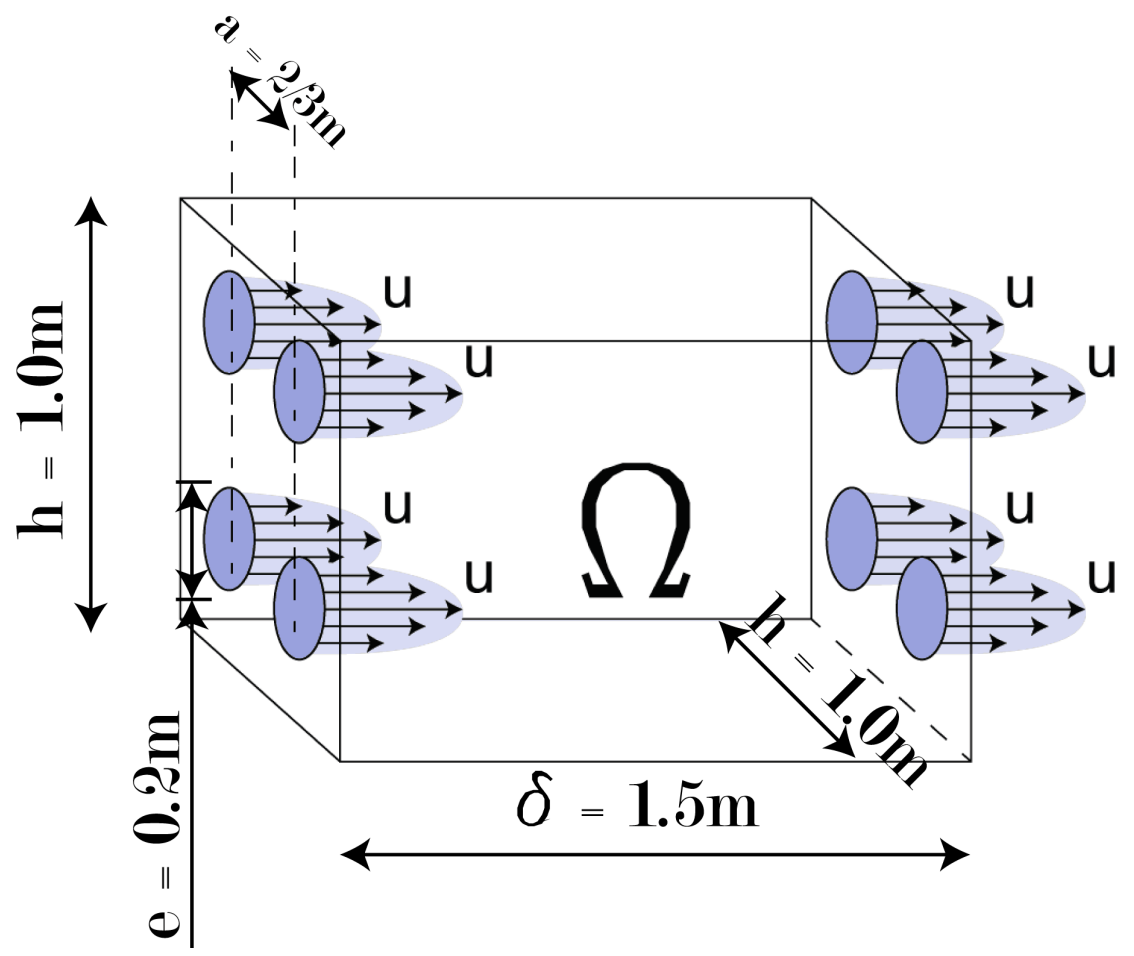

Figure 59: 3d design domain with $\mathbf{u}$ according to $R e$ value.

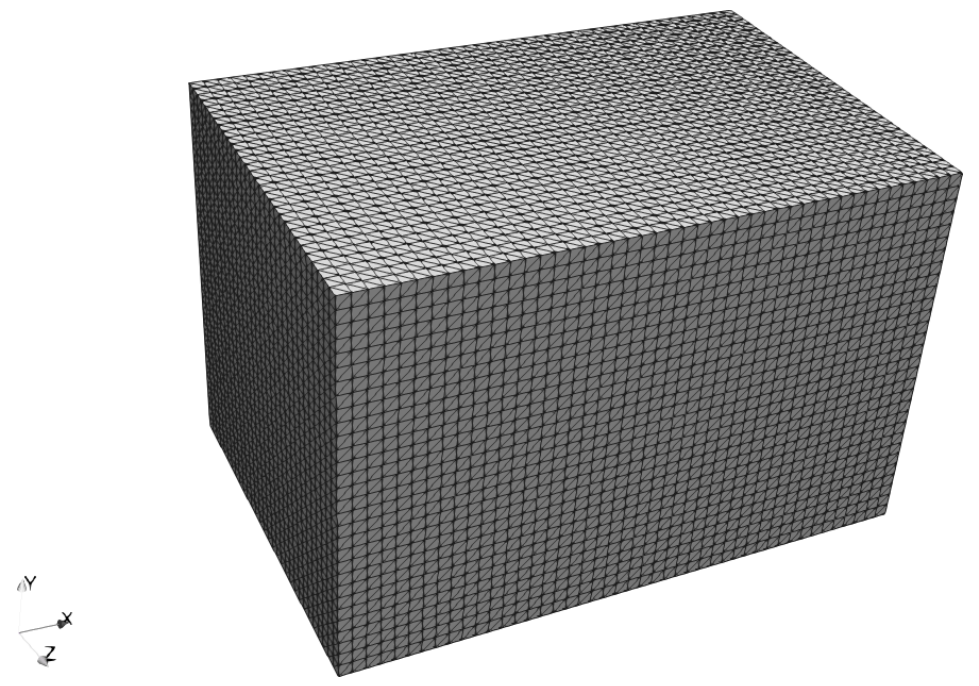

Figure 60: Mesh discretization of the 3D problem (243000 elements).

The intermediary topologies of the problem are shown in Figure 61, which starts with a fully fluid domain and proceed to merge the channels, similarly to the behavior of the double-channel example. It is interesting to see how a more complex geometry behave with the technique presented in this work. In this case, the four inlets merge into a unique 
pipe in the middle of the design domain, thus the energy dissipation is reduced due to smaller surface area inside pipes.

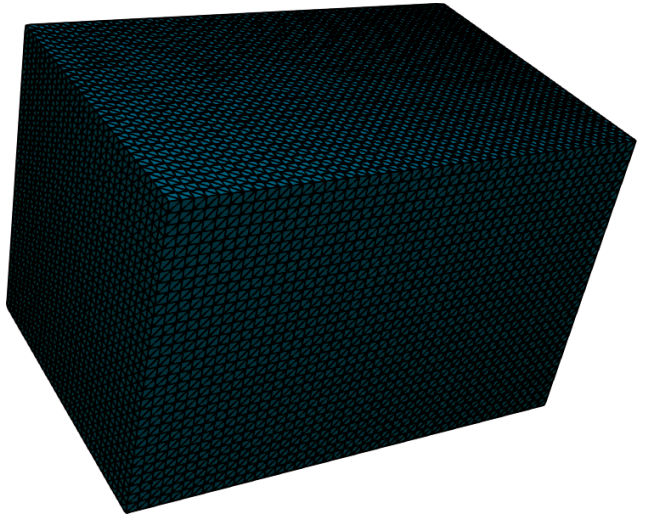

(a) Iteration number: 0

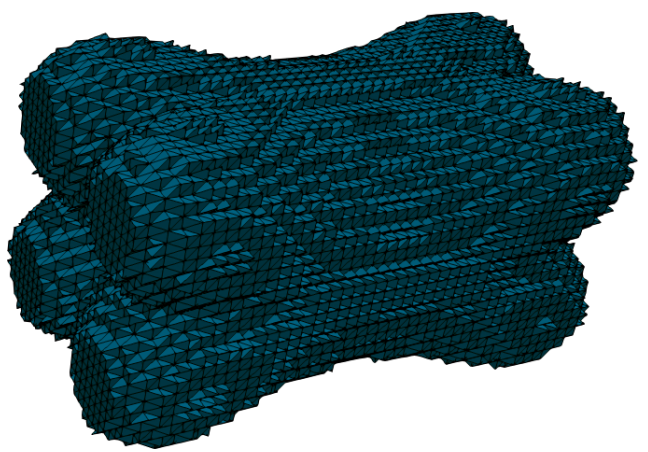

(c) Iteration number: 25

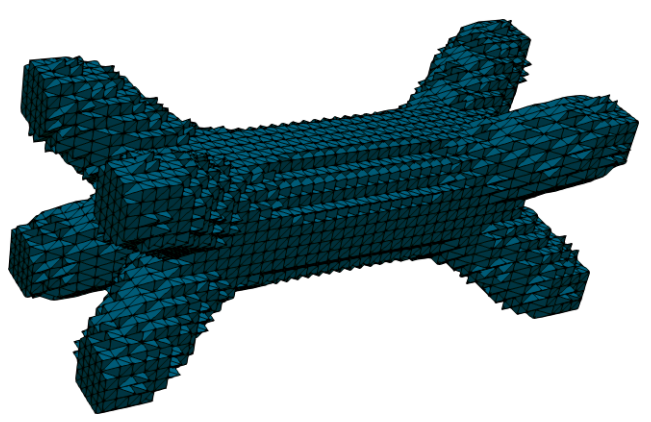

(e) Iteration number: 100

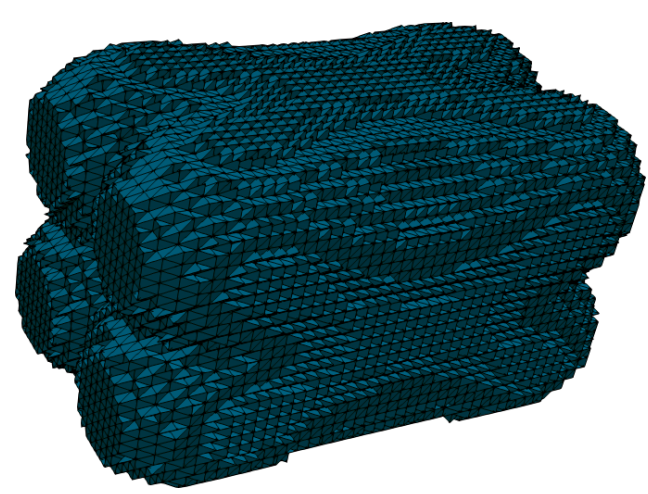

(b) Iteration number: 10

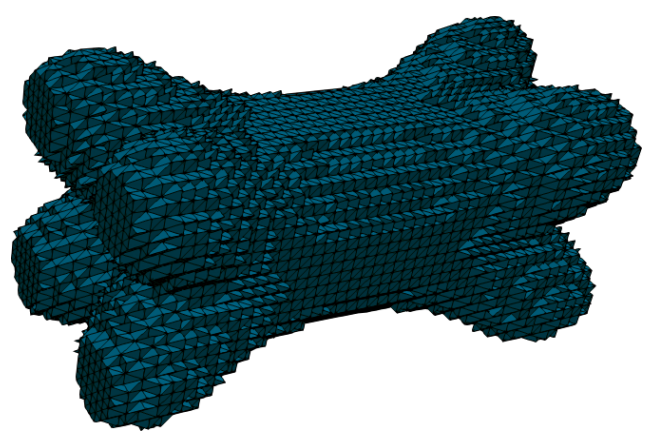

(d) Iteration number: 50

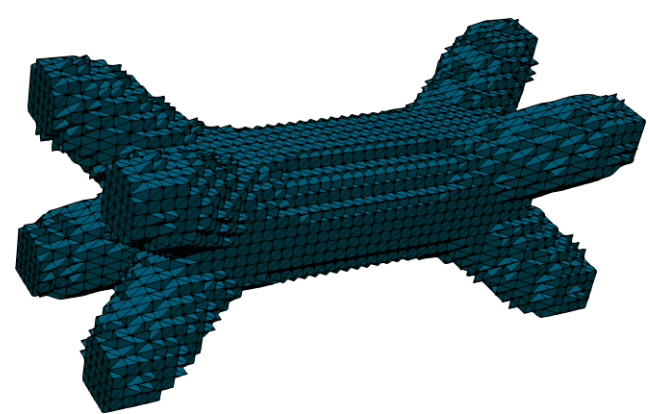

(f) Iteration number: 240

Figure 61: Results evolution during optimization in the 3D design domain.

The convergence plot is shown in Figure 62 . 


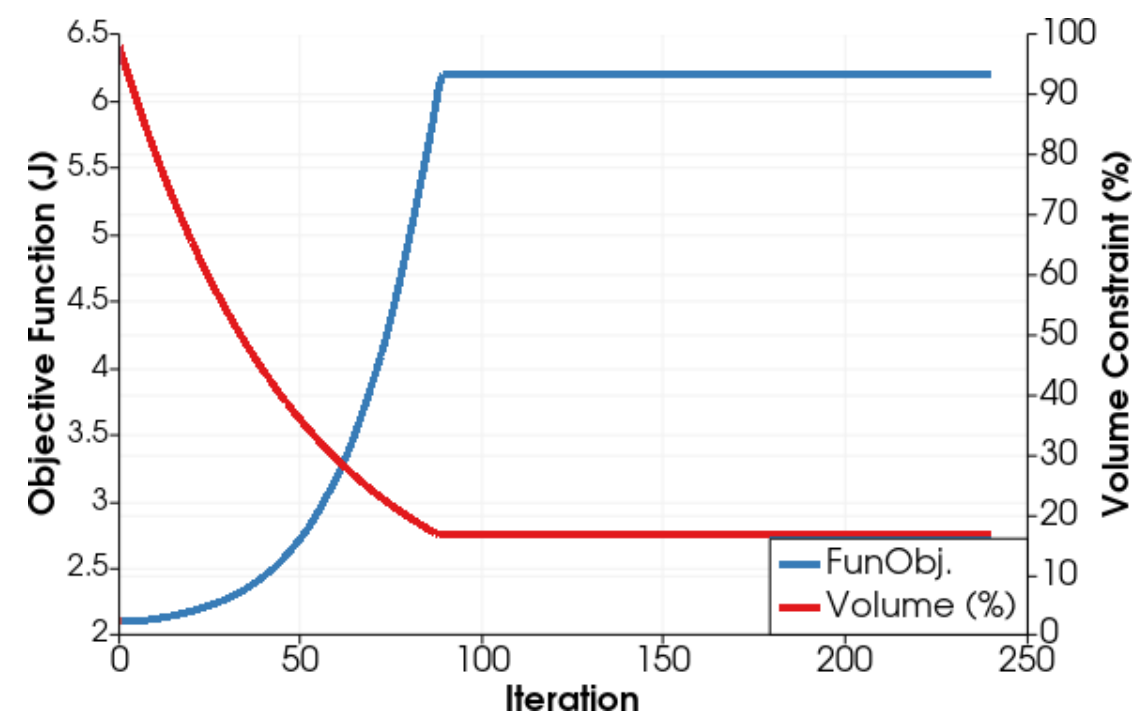

Figure 62: Convergence plot of the objective function in the 3D design domain.

\subsection{Fluid diodes}

The previous examples solve dissipation minimization problems as benchmarks. This subsection now introduces the diodicity problem illustrated in Figure 63. The main idea is to facilitate the flow in one direction and to inhibit the fluid flow in the reverse direction. The applied objection function (Eq.3.8) and the boundary condition (including pressure on outlet) represents the diodicity and it is the same used in literature [LIN et al., 2015]. The finite elements are illustrated in Figure 64 with 7200 elements considering the applied symmetry.

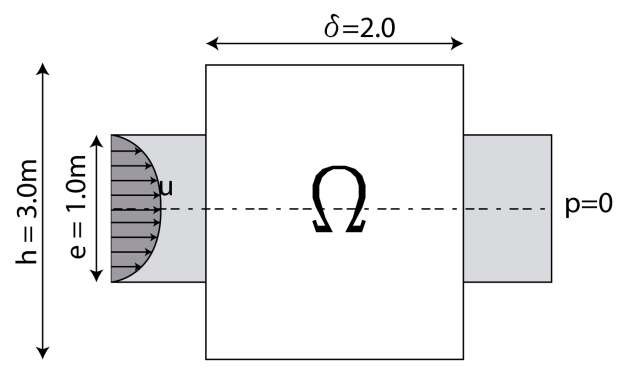

(a) Main Flow

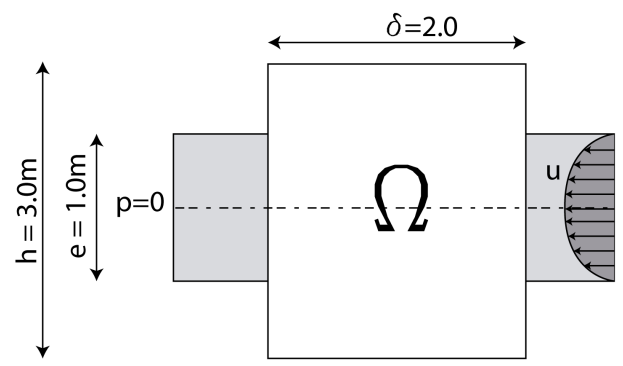

(b) Reverse Flow

Figure 63: Design domain of the fluid diode problem with $\mathbf{u}$ according to $R e$ value. 


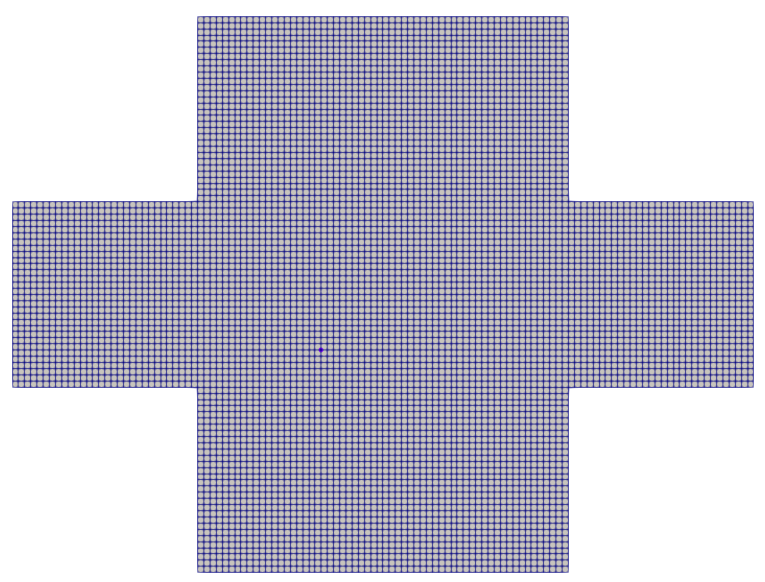

Figure 64: Mesh discretization of the fluid diode problem (7200 elements).

To show one advantage of the method, which is elimination of gray scale, the fluid diode optimization is performed by using the traditional density method with standard properties described in Table 4 including the usual continuation in the $q$ factor. Figure 65 shows the evolution of the topology optimization using the traditional method (with Interior Point Optimizer) and also the undesirable gray scale. The literature [LIN et al., 2015] had to change this default values and also to insert a filter to obtain a reasonable 0-1 solution. However, depending on the problem, these settings can be hard to obtain with many trial and errors.

Table 4: Parameters used in the fluid diode optimization

\begin{tabular}{cc}
\hline Property & Value \\
\hline Re & 300 (inlet) \\
$\mathrm{q}$ & $0.01->0.10$ \\
$\kappa_{\max }$ & $2.5 \cdot 10^{4}$ \\
$\kappa_{\min }$ & 0 \\
\hline
\end{tabular}




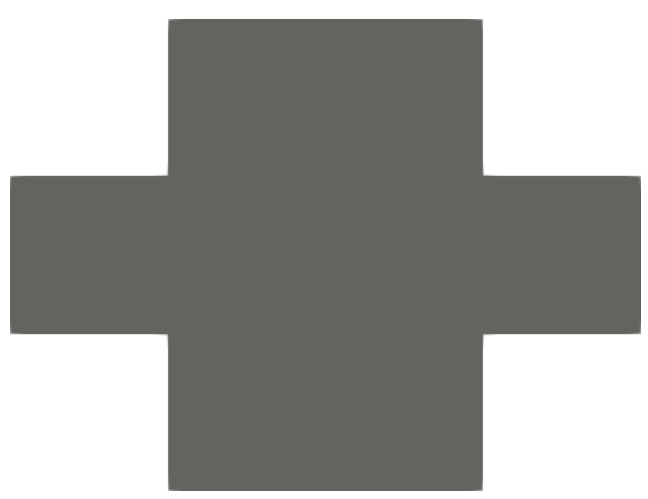

(a) Iteration number: 0

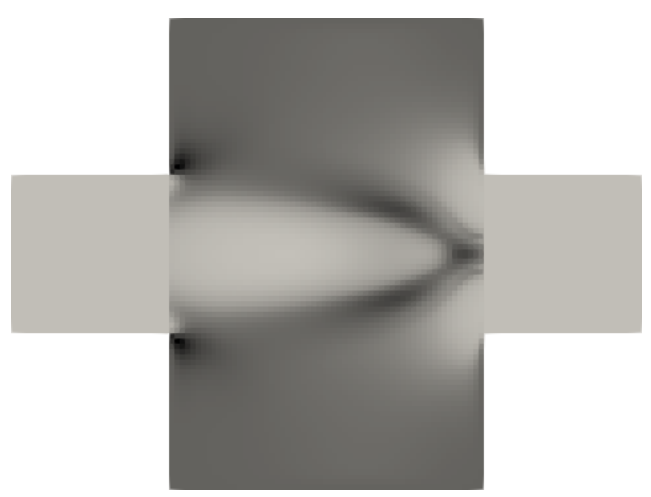

(c) Iteration number: 100

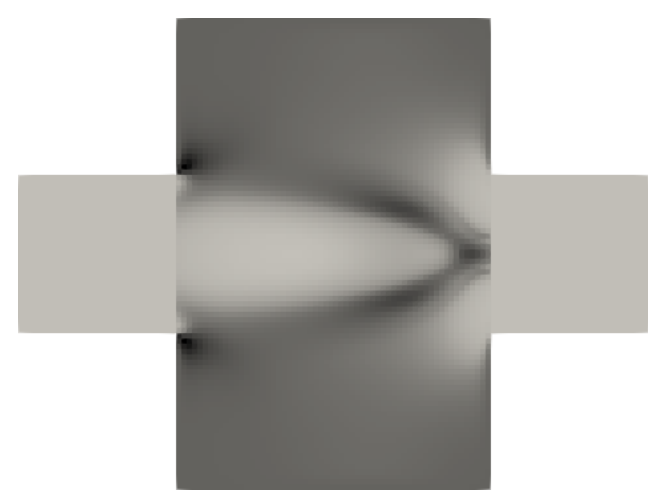

(b) Iteration number: 25

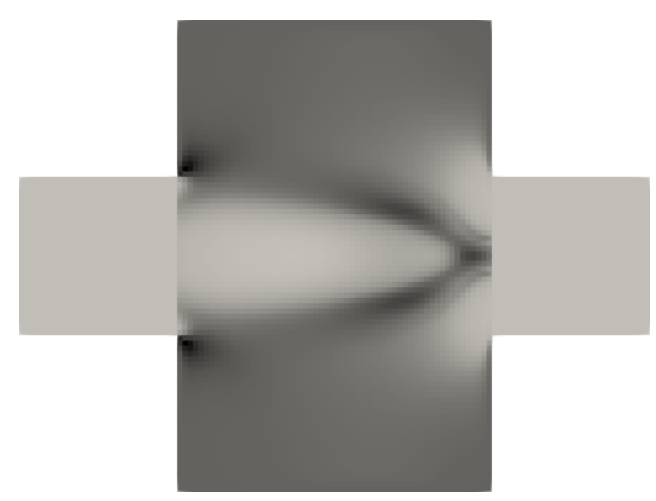

(d) Iteration number: 240

Figure 65: Results evolution during optimization using traditional density method.

On the other hand, the proposed binary method can create only 0-1 solutions and the results are illustrated in Figure 66. Since this problem is complex to understand its behaviour, Figure 67 shows the velocity magnitude of both (a) main and (b) reverse flow, indicating greater dissipation in the second case. In such simulation, it is easy to find small portions of fluid flowing through solid parts (see original work of [LIN et al., 2015]) and the extracted geometry can check if the diodicity actually occurs or not. For this reason, the velocity field of extracted geometry is plot in Figure 68 showing very similitude to the Figure 67 and the calculated diodicity is 1.16 and 1.13, respectively.

In the TOBS method, $q$ factor is set to $1, \kappa_{\max }$ is set to $8 \cdot 10^{3}$ and all remaining values are kept the same. The $\kappa_{\max }$ value can be changed and other local minima can be achieved. 


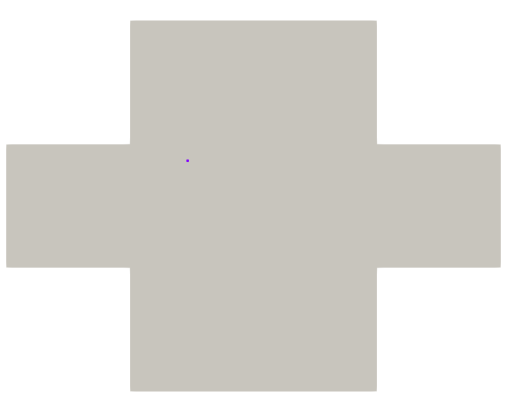

(a) Iteration number: 0

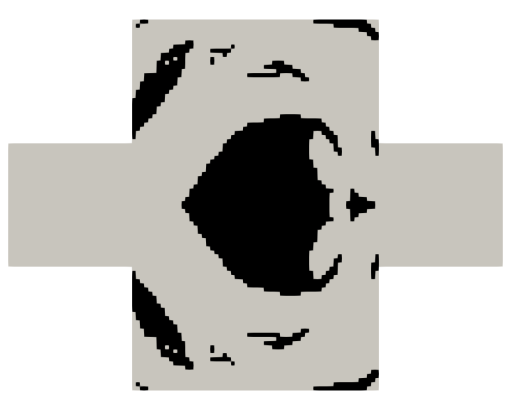

(c) Iteration number: 50

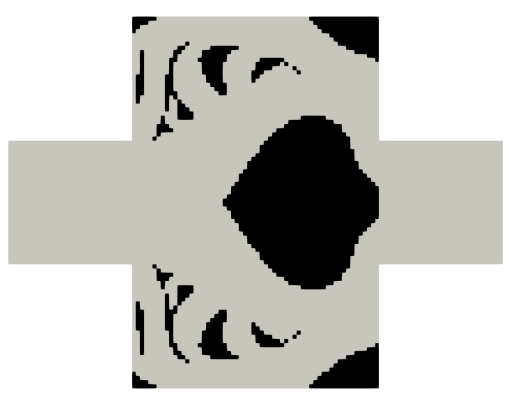

(e) Iteration number: 100

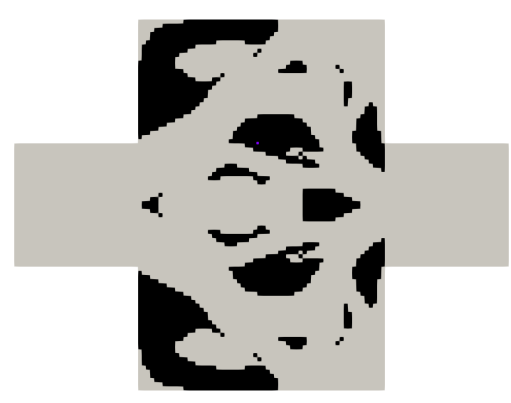

(b) Iteration number: 25

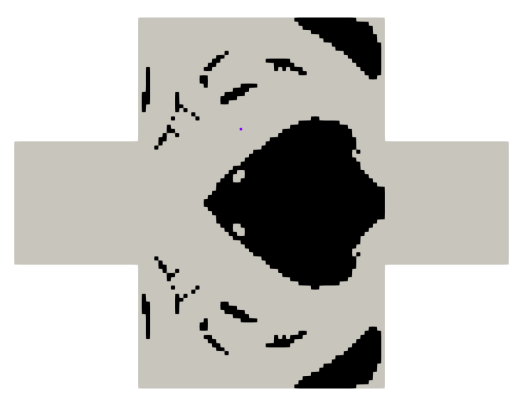

(d) Iteration number: 75

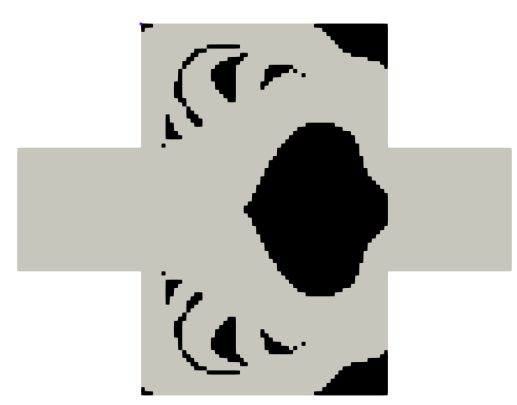

(f) Iteration number: 240

Figure 66: Results evolution during optimization using binary approach.

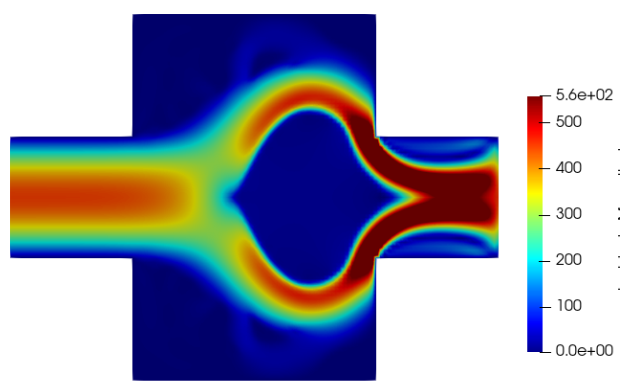

(a) Main flow

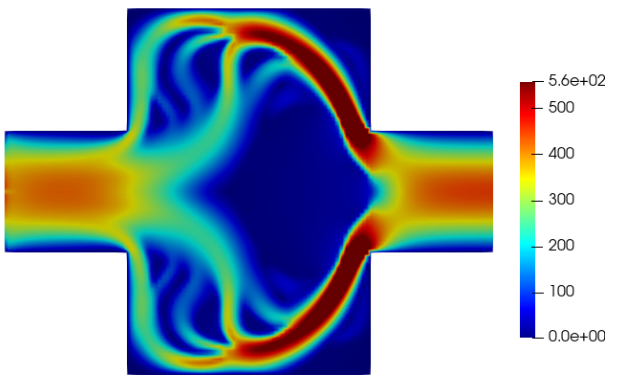

(b) Reverse flow

Figure 67: Fluid flow simulation of the optimized result with material model. 


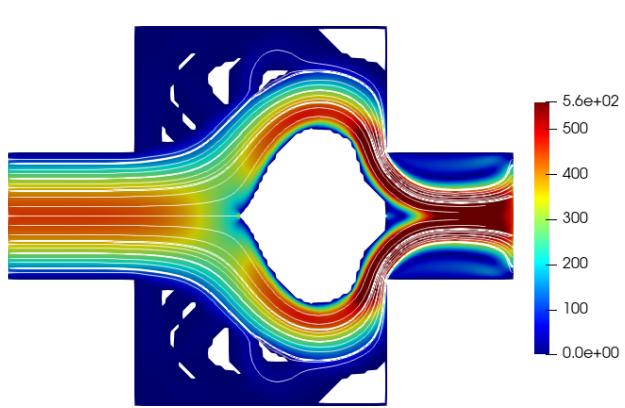

(a) Main flow

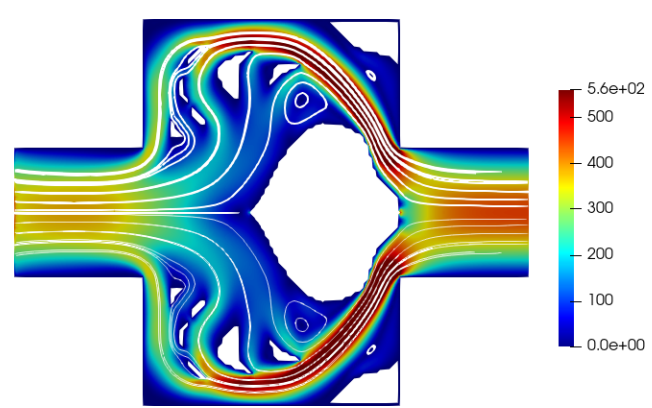

(b) Reverse flow

Figure 68: Fluid flow simulation of extracted geometry.

Also, larger fluid diodes are also shown in Figure 69a-b in order to explore different aspect ratios. The velocity plots can clearly show the difference of forward flow (Figure 70a and 71a) and reverse flow (Figure 70b and 71b).

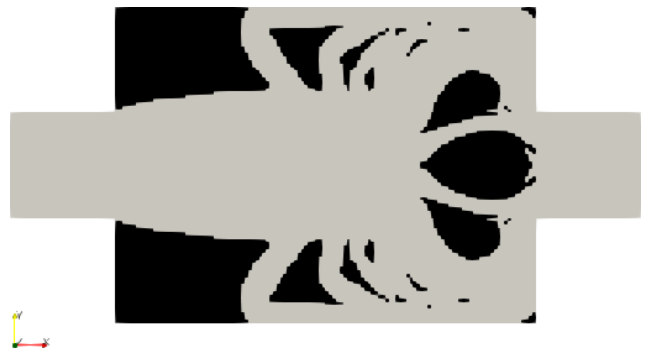

(a) Aspect ratio of 4:3

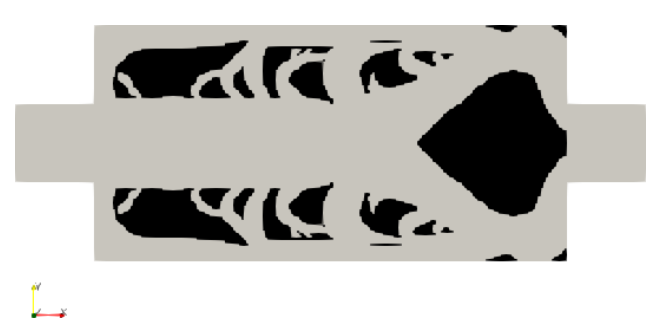

(b) Aspect ratio of 6:3

Figure 69: Fluid flow simulation.

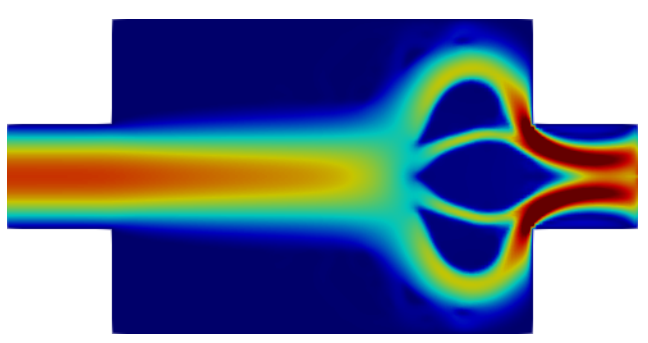

(a) Main flow

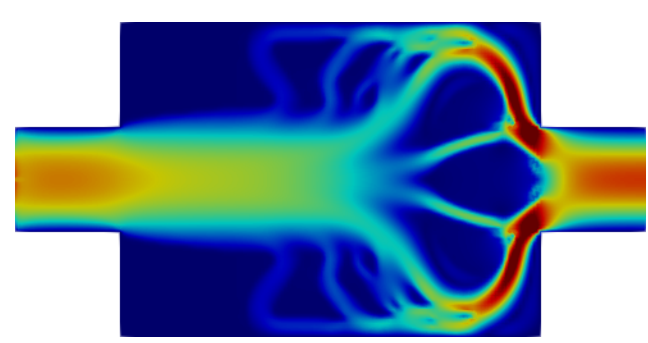

(b) Reverse flow

Figure 70: Fluid flow simulation for ratio aspect of 4:3.

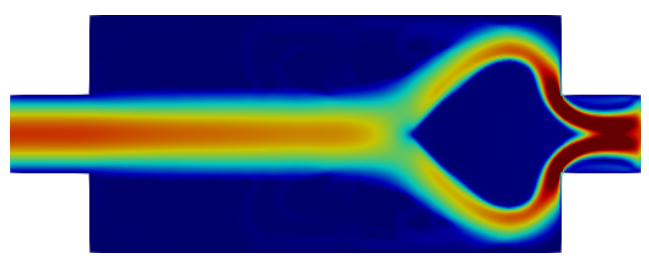

(a) Main flow

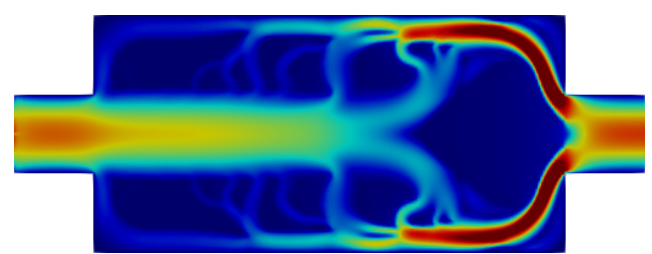

(b) Reverse flow

Figure 71: Fluid flow simulation.for ratio aspect of 6:3. 
The effectiveness of presented geometries can be seen as diodicity in Table 5, according to the aspect ratio.

Table 5: Diodicity Values for optimized geometries.

\begin{tabular}{cc}
\hline Aspect ratio & Diodicity \\
\hline $2: 3$ & 1.13 \\
$4: 3$ & 1.22 \\
$6: 3$ & 1.33 \\
\hline
\end{tabular}

\subsubsection{D swirl fluid diodes design considering diodicity}

From this section onwards, the approach is related to labyrinth seals. When facing special fluid diodes such as labyrinth seals, it is important to model a 2D swirl space and also force the energy dissipation in one direction, which in this work is defined as right to left. The results shown in next sections uses the design domain and boundary conditions illustrated in Figure 72.

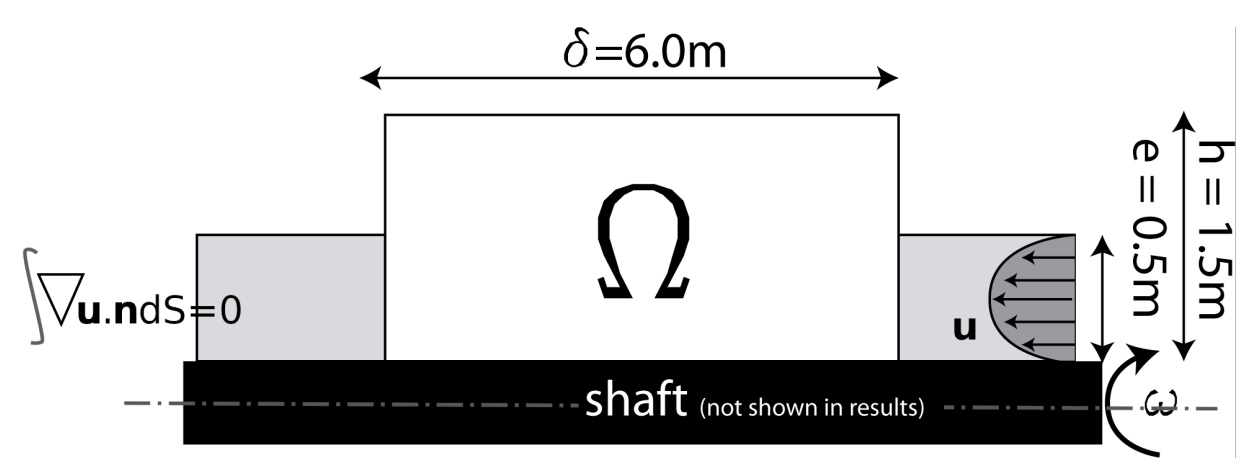

Figure 72: Labyrinth Seal design domain and boundary conditions with $\mathbf{u}$ based on $R e$ number.

The parameters of this simulation can be seen in Table 6, with a initial guess full of fluid in the design domain. 
Table 6: Optimization parameters in the 2D swirl model

\begin{tabular}{cc}
\hline parameters & value \\
\hline$d$ & $40 \mathrm{~mm}$ \\
$R e$ & 200 \\
$n$ & $100 \mathrm{rpm}$ \\
$r_{\min }$ & $0.10 \mathrm{~mm}$ \\
$\kappa_{\text {max }}$ & $10^{9}$ \\
$W_{F}$ & 0.1 \\
$W_{E}$ & 0 \\
$W_{V}$ & 0 \\
$V_{u p}$ & $80 \%$ \\
$V_{\text {down }}$ & $50 \%$ \\
\hline
\end{tabular}

The topology optimization results of an approach considering only diodicity can be seen in Figure 73. The velocity plot in this work focuses on the $2 \mathrm{D}$ plot, since the perpendicular velocity is very high and can be hard to understand the full behaviour. With this in mind, looking Figure 74, it is possible to see a microchannel almost closing the passage, which is not acceptable for labyrinth seals, because the fixed parts may not touch the shaft. Figure 75 shows the circumferential velocity evidencing parts attached to shaft and Figure 76 shows the pressure plot. The convergence plot of this simulation is shown in Figure 77.

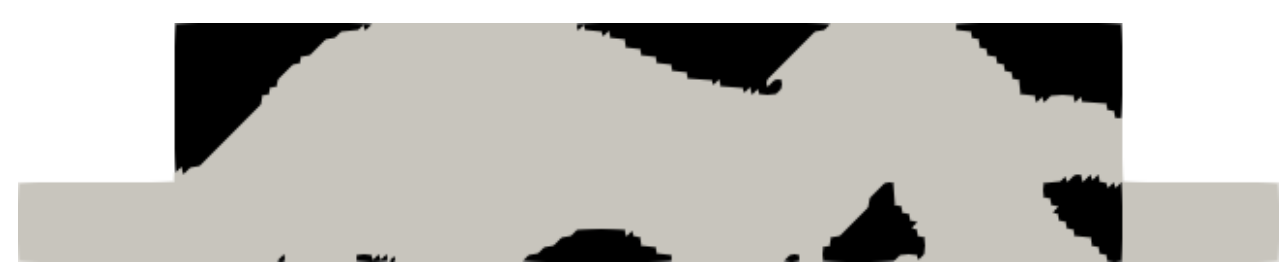

Figure 73: Geometry of a labyrinth seal using only diodicity. 


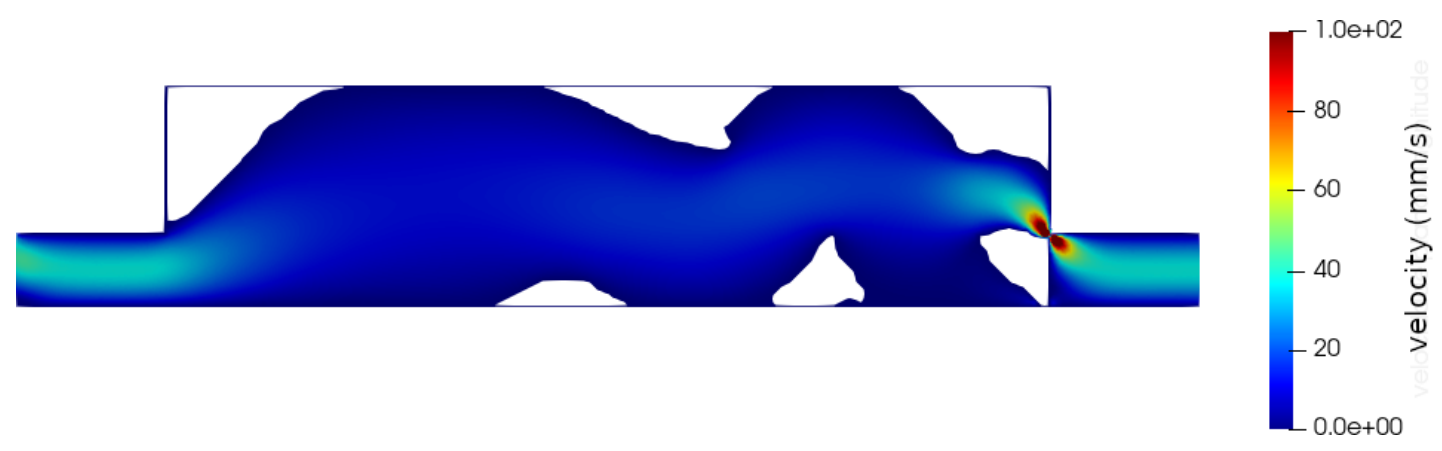

Figure 74: Modulus of plane velocity (flow from right to left) of geometry obtained with diodicity approach.

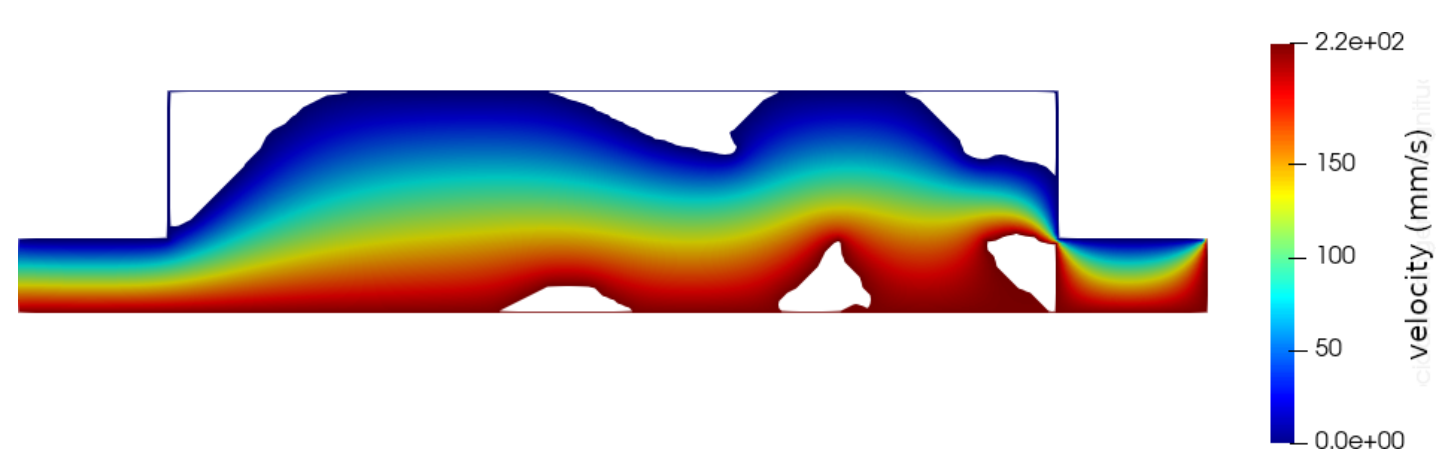

Figure 75: Circumferential velocity plot of geometry obtained with diodicity approach.

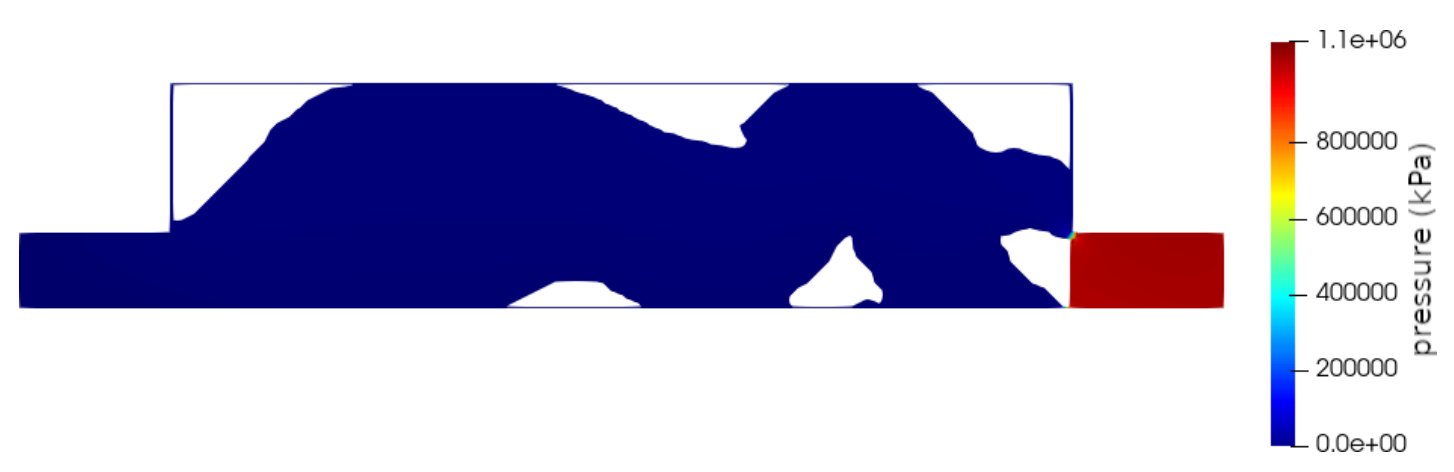

Figure 76: Pressure field of geometry obtained with diodicity approach. 


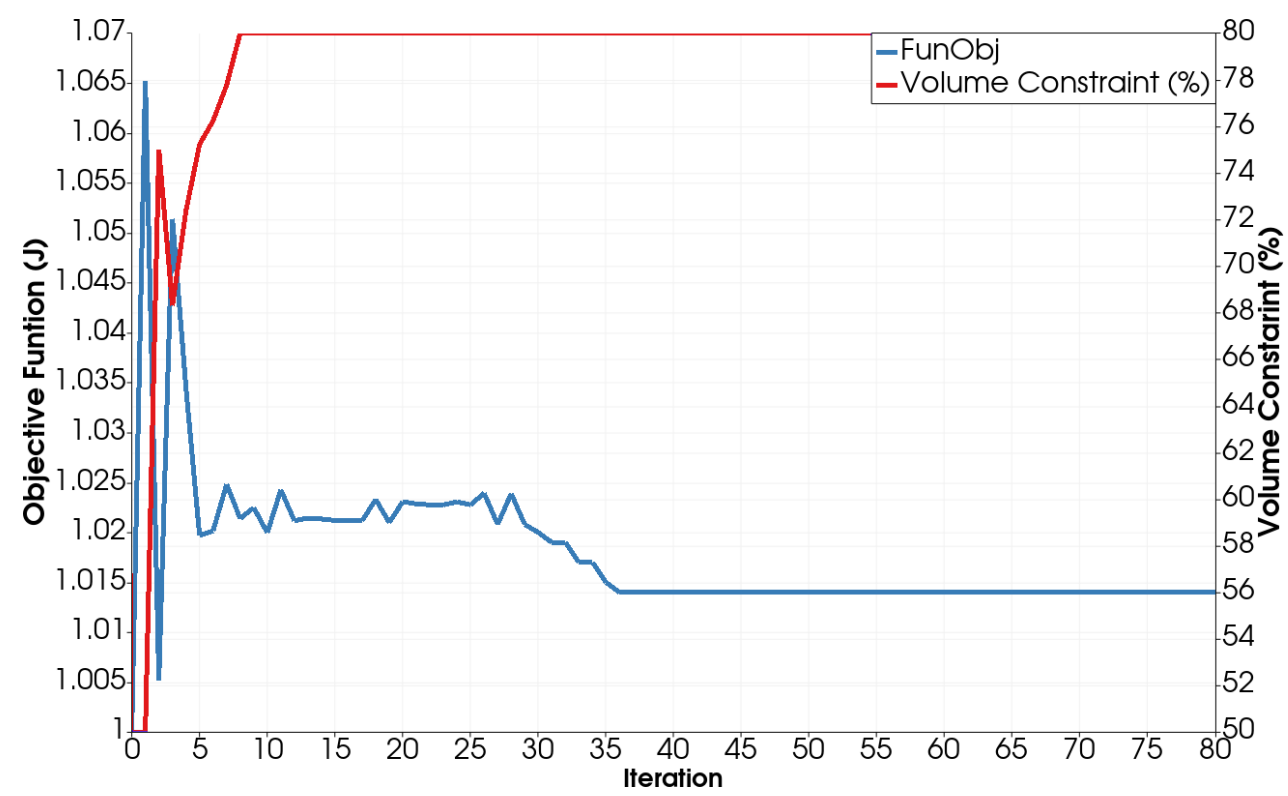

Figure 77: Objective function of diodes in a 2Dswirl system.

For this reason, the parameter $W_{F}$ is raised to 3 and the result is shown in Figure 78. Despite the problem with closing passage is now solved, the geometry and effectiveness of the seal is not plausible (see Figure 79). Figures 79 and 80 shows the plane and circumferential velocity, respectively, and Figure 81 shows the pressure plot.

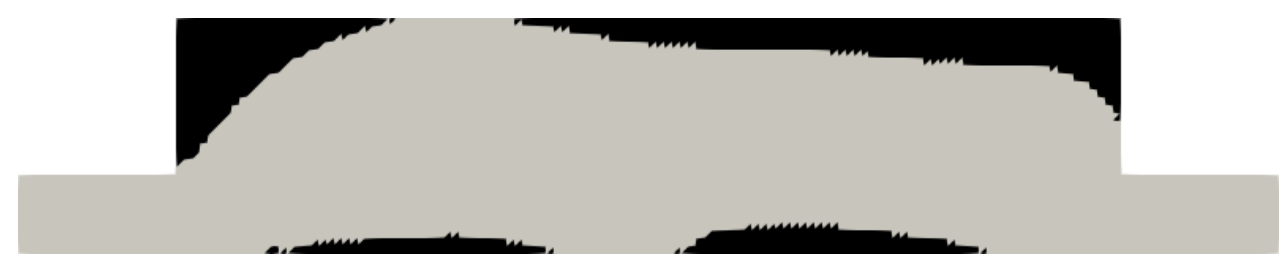

Figure 78: Geometry with raised $W_{F}$ value.

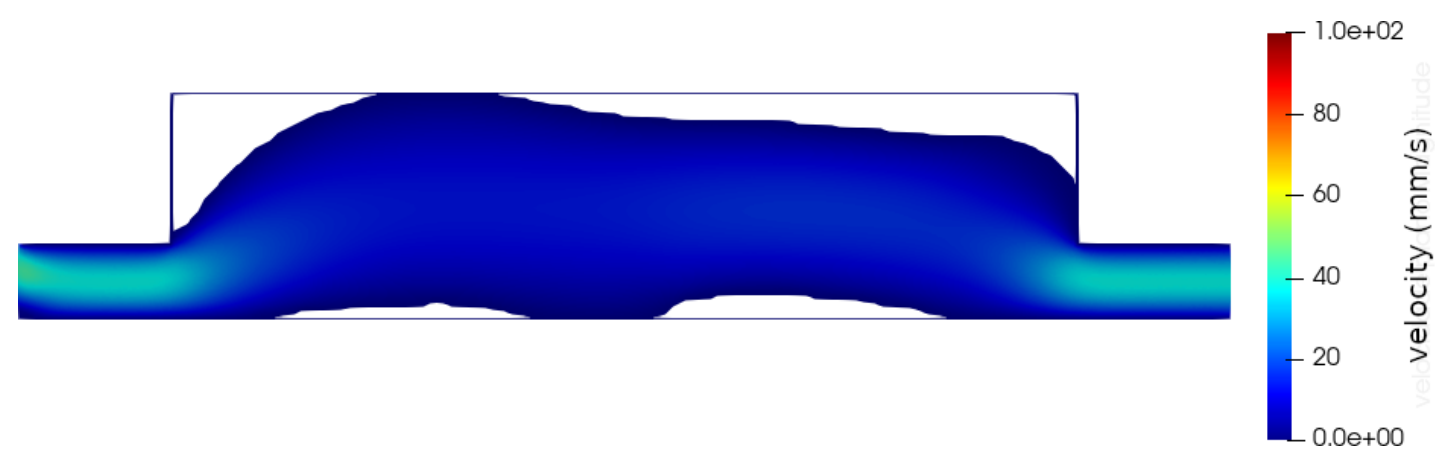

Figure 79: Modulus of plane velocity of geometry with high $W_{F}$ value. 


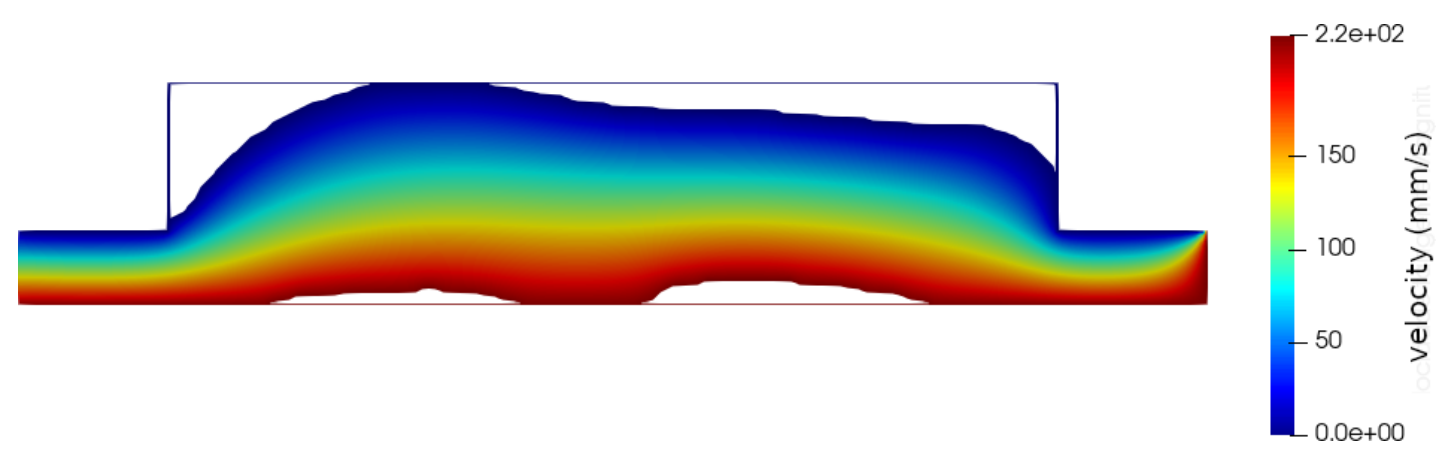

Figure 80: Circumferential velocity plot of geometry with high $W_{F}$ value.

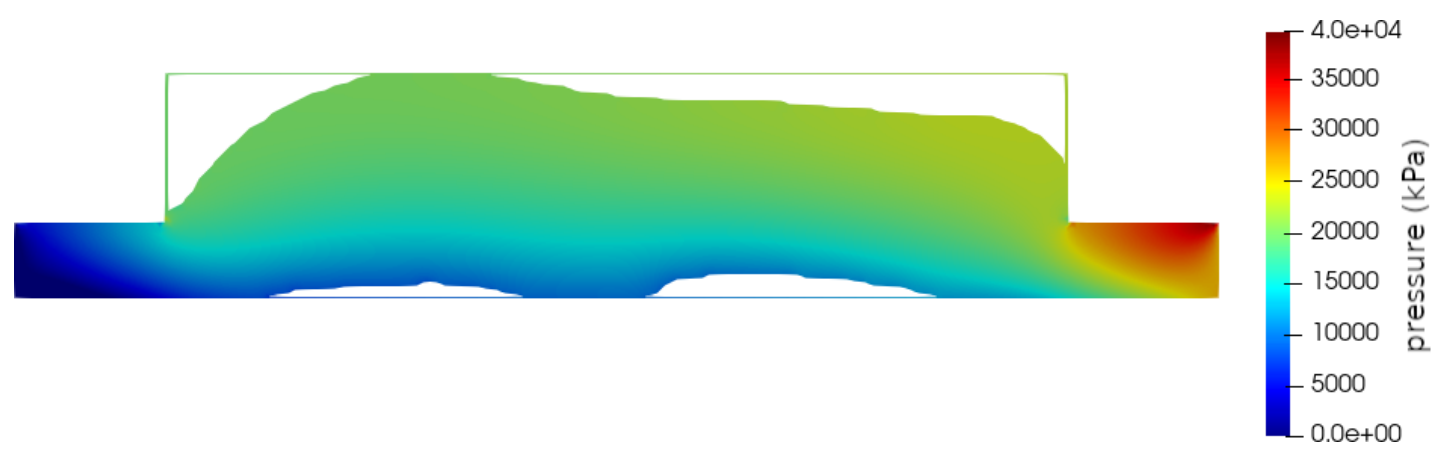

Figure 81: Pressure field of geometry with high $W_{F}$ value.

One good approach to solve this issue, it is to keep low $W_{F}$ value and to restrain the design domain on both left and right side with the size of the gap length (see Figure 82). Thus, the obtained geometry can be seen in Figure 83 with velocity plot in Figure 84.

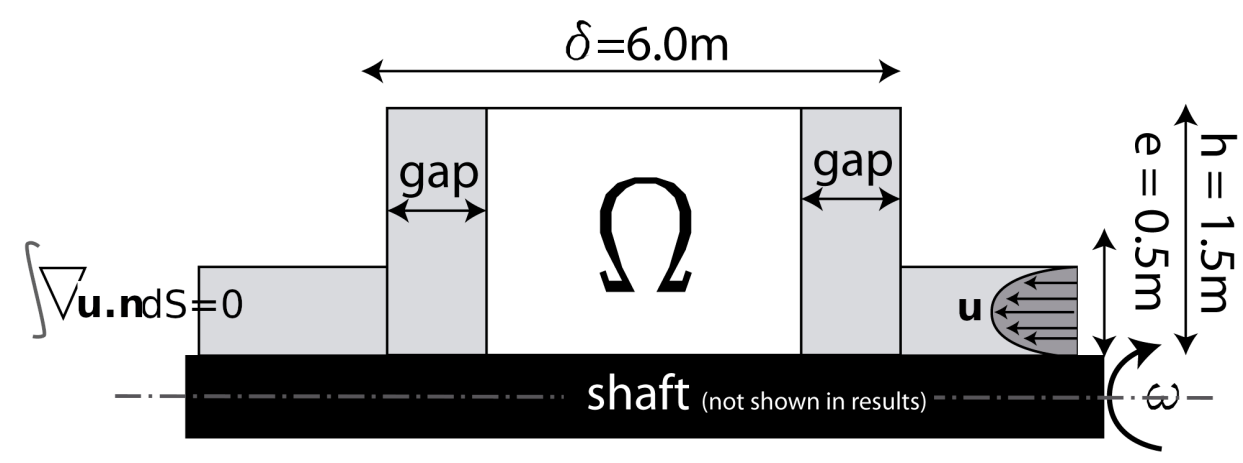

Figure 82: Restricted design domain of labyrinth seals.

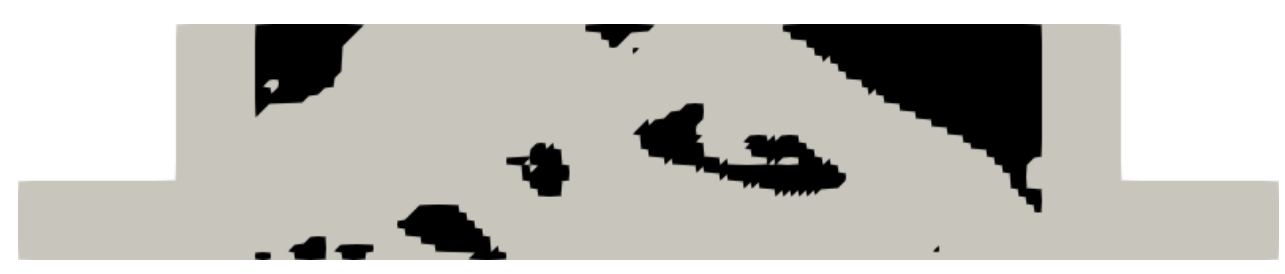

Figure 83: Geometry of a labyrinth seal with a smaller design domain. 


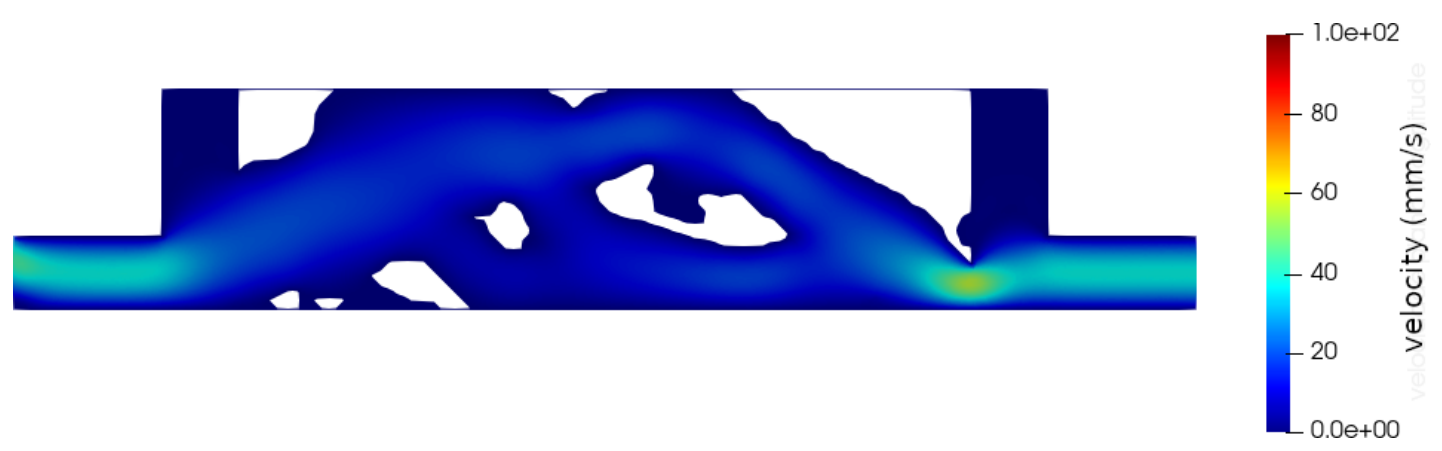

Figure 84: Modulus of plane velocity of a labyrinth seal with smaller design domain.

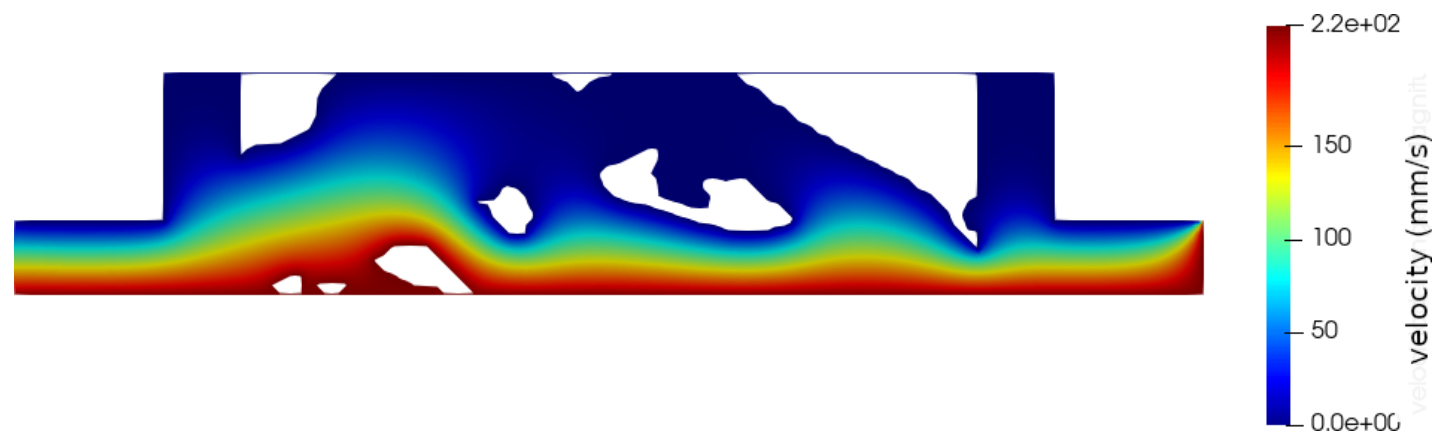

Figure 85: Circumferential velocity of a labyrinth seal with smaller design domain.

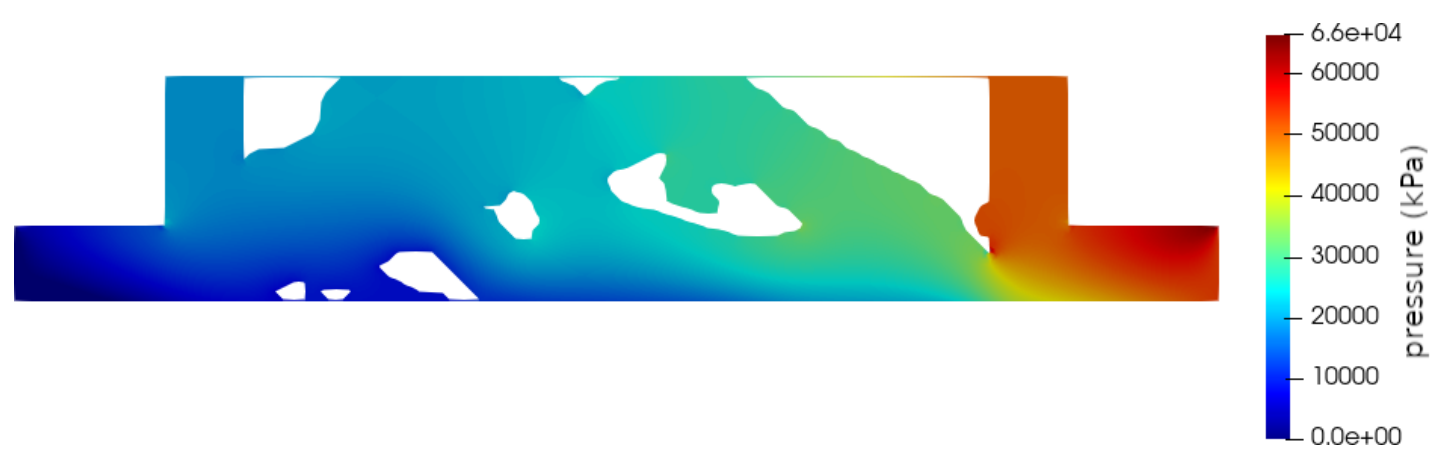

Figure 86: Pressure field of a labyrinth seal with smaller design domain.

Figure 83 also shows the undesirable floating islands in the middle of the design domain, which is very hard to implement, since it does not have any support from stator or the shaft. Figures 84 and 85 shows the plane and circumferential velocity, respectively and Figure 86 shows the pressure plot.

To explore more possible geometries, another simulation is performed with a filter radius of $r_{\min }=0.05$ and interesting geometries are shown in Figure 87 with plane and perpendicular velocities plot in Figures 88 and 89, respectively. Pressure field is shown in Figure 90 . 


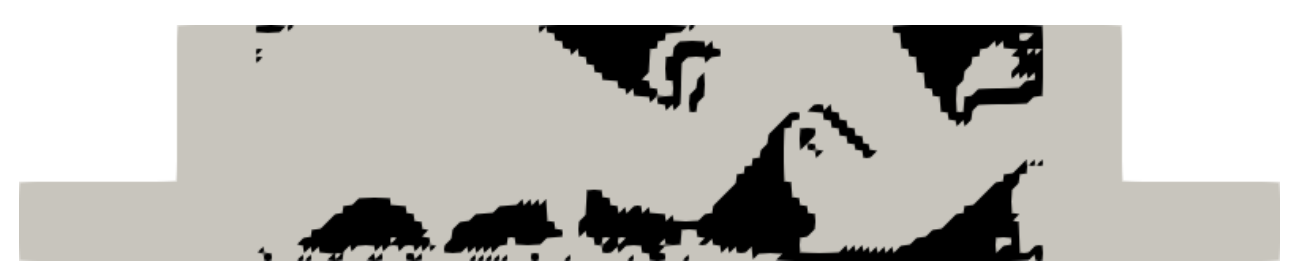

Figure 87: Modified design domain with $r_{\min }=0.05$.

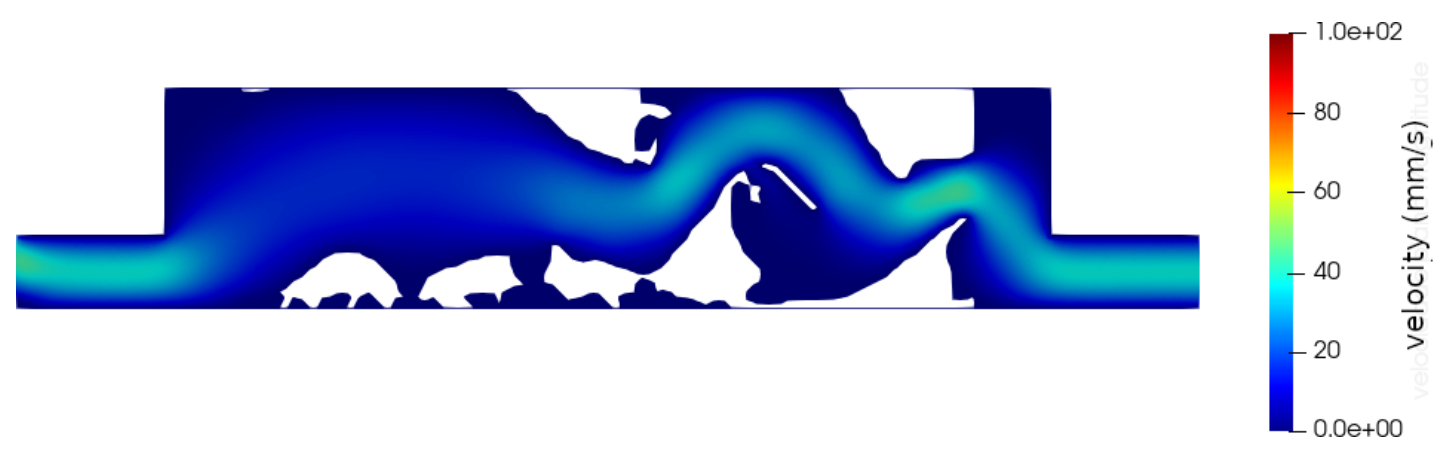

Figure 88: Modulus of plane velocity of design domain with $r_{\min }=0.05$.

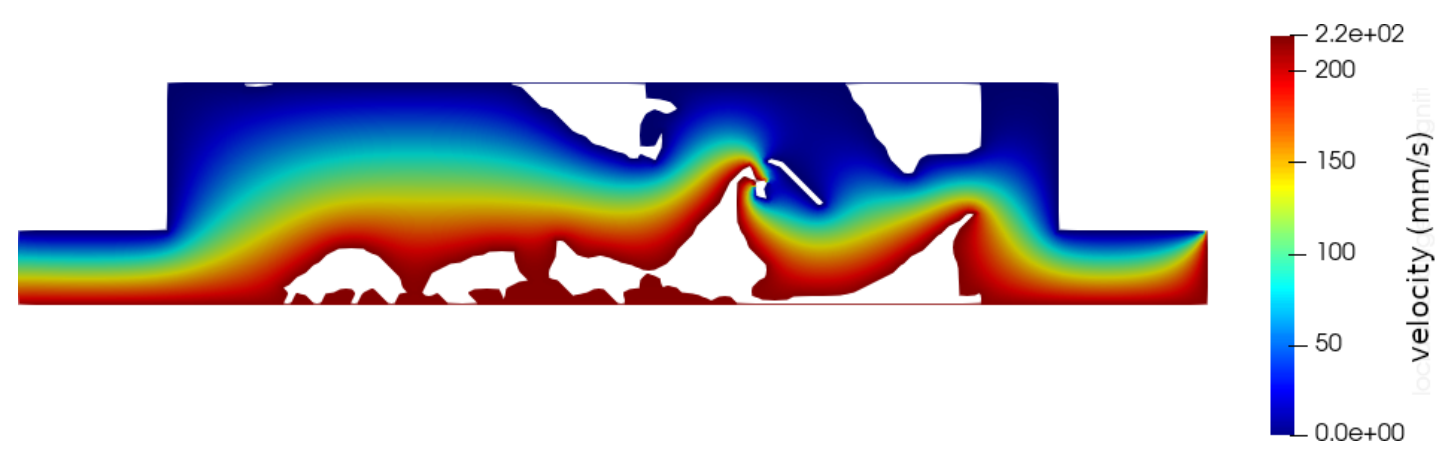

Figure 89: Circumferential velocity of design domain with $r_{\min }=0.05$.

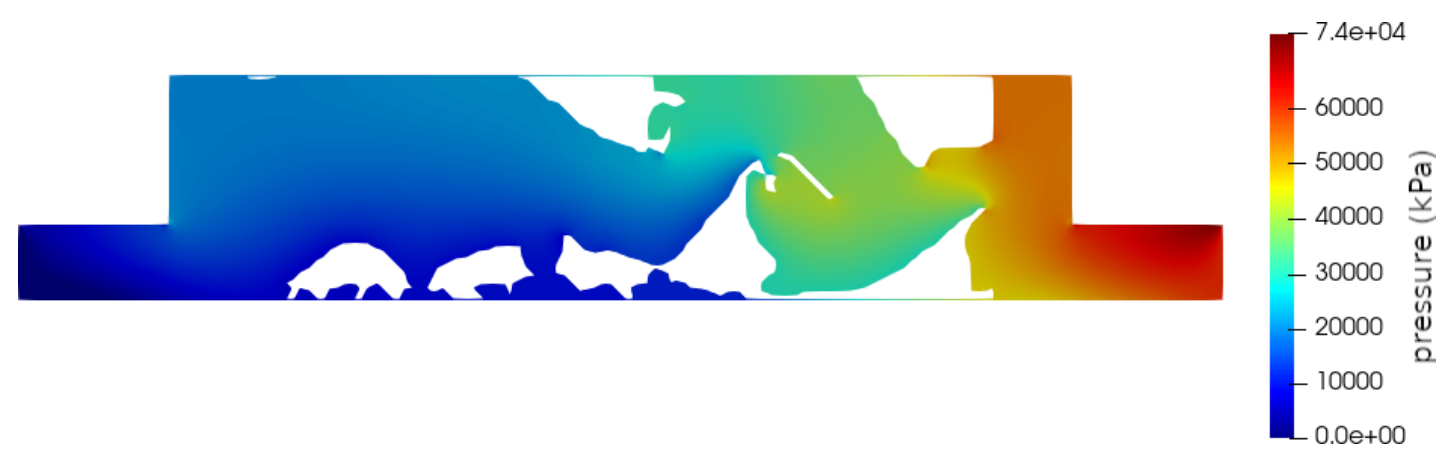

Figure 90: Pressure field of design domain with $r_{\text {min }}=0.05$.

Besides, the $\kappa_{\max }$ is changed to $10^{10}$ and a different geometry is shown in Figure 91. The plane and circumferential velocity plot in Figures 92 and 93, respectively show also 
that there are some spots that have no significant plane velocity, in other words that region could be eliminated. Pressure field is plot in Figure 94

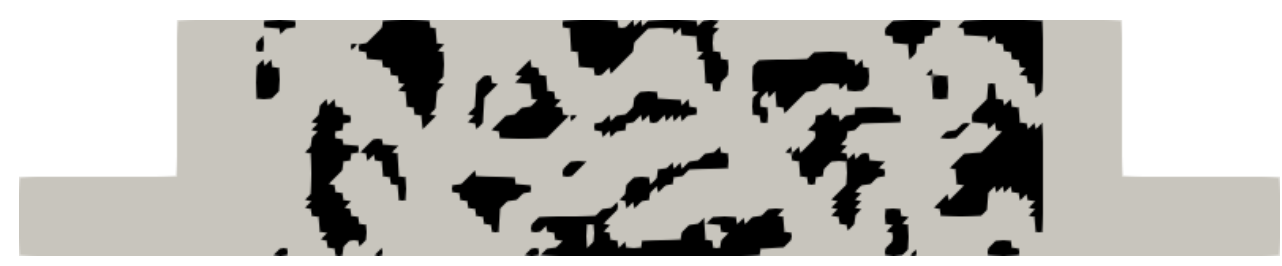

Figure 91: Modified design domain with $\mathrm{kmax}=10^{10}$.

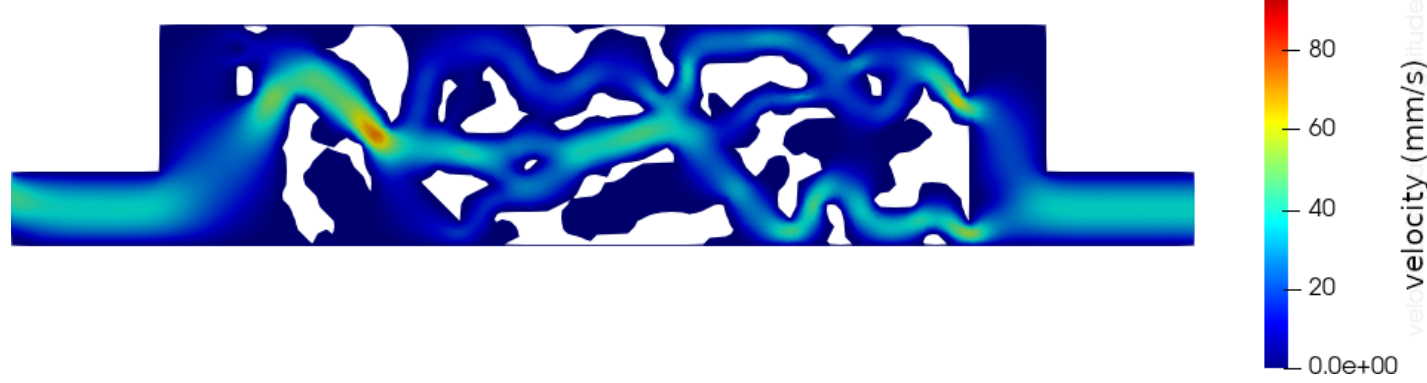

Figure 92: Modulus of plane velocity with $\mathrm{kmax}=10^{10}$.

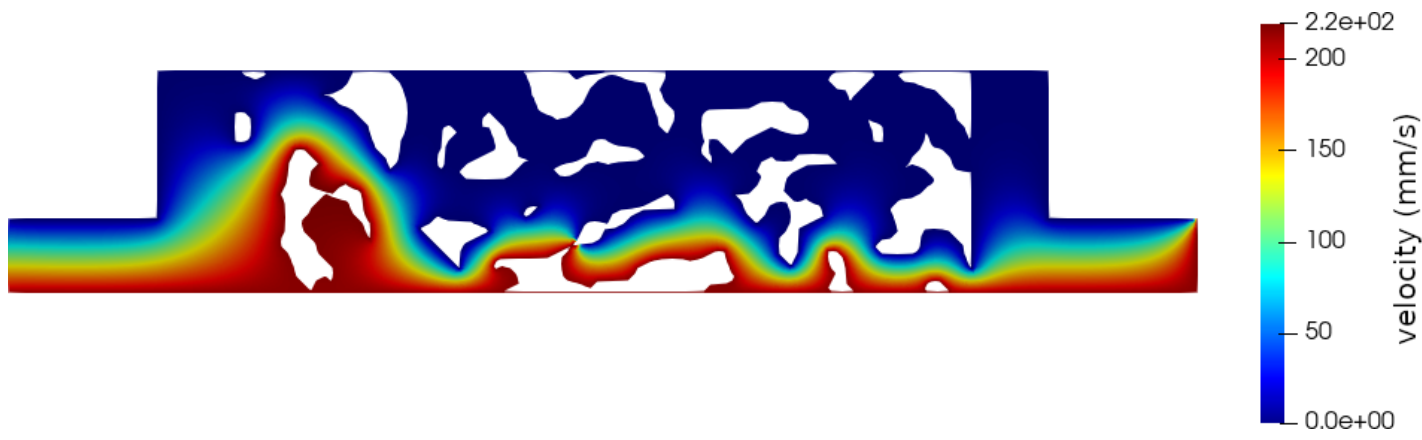

Figure 93: Circumferential velocity plot with $\mathrm{kmax}=10^{10}$.

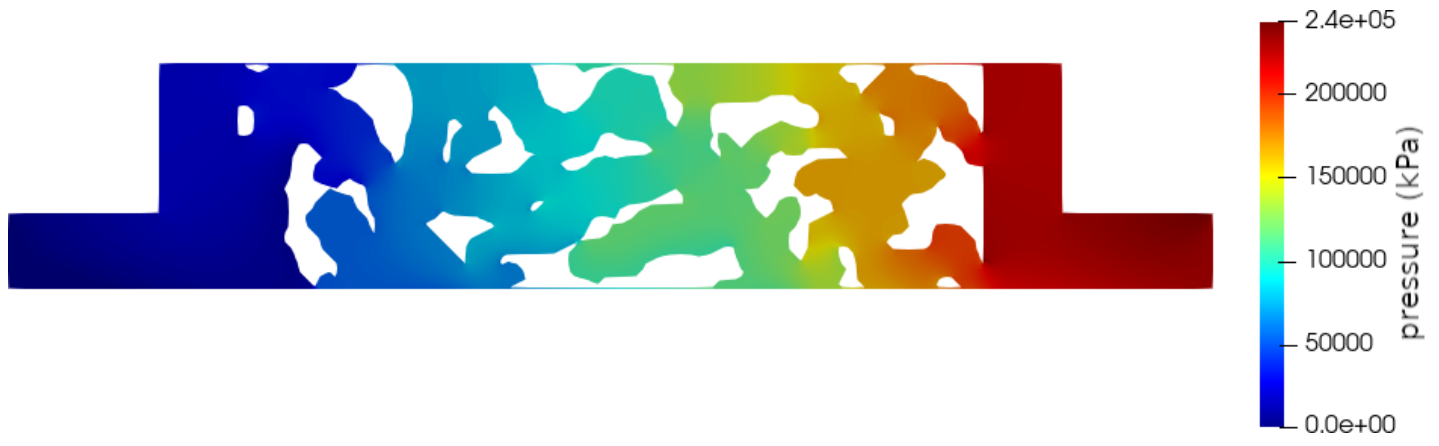

Figure 94: Pressure field with $\mathrm{kmax}=10^{10}$. 
In the same analysis, the filter radius is raised to 0.20 and a reasonable solution is shown in Figure 95 with velocity plot in Figure 98.

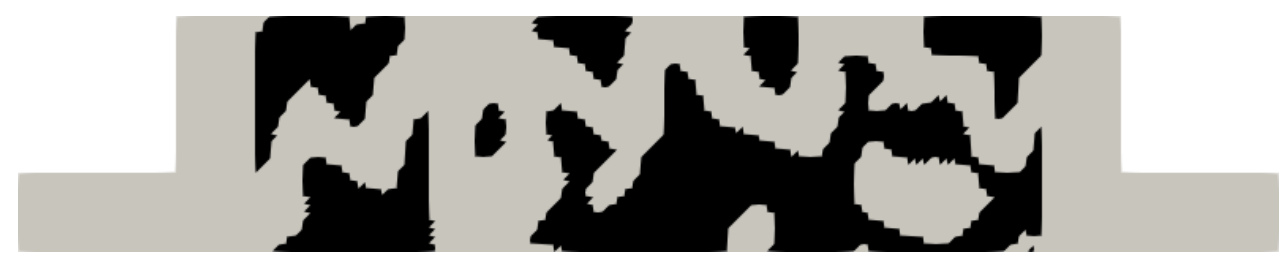

Figure 95: Modified design domain with $\mathrm{kmax}=10^{10}$ and $r_{\min }=0.20$.
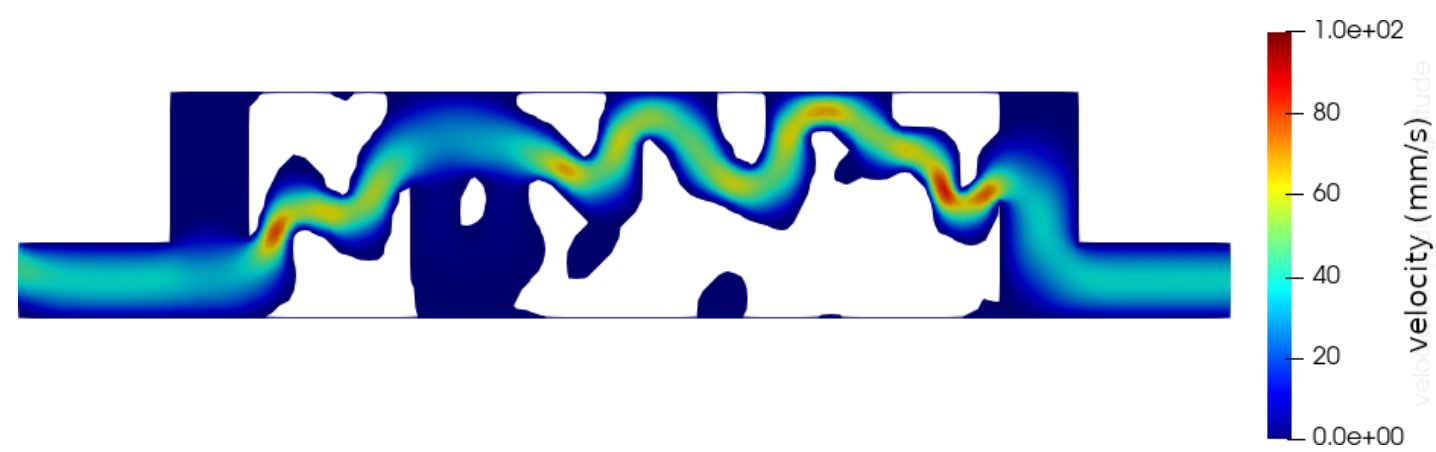

Figure 96: Modulus of plane velocity of modified design domain with $\mathrm{kmax}=10^{10}$ and $r_{\text {min }}=0.20$.

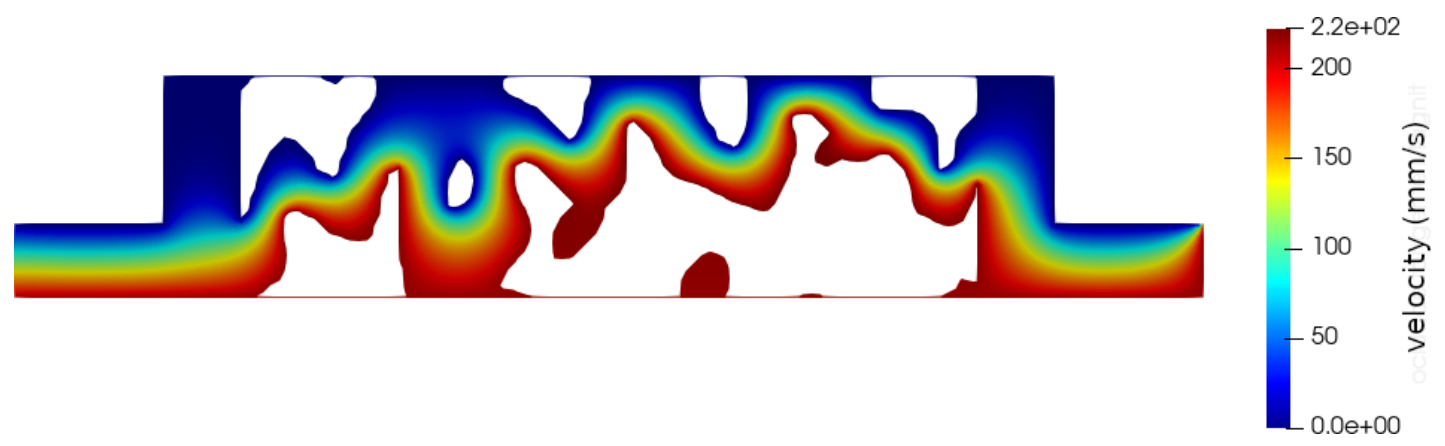

Figure 97: Circumferential velocity plot of modified design domain with $\mathrm{kmax}=10^{10}$ and $r_{\text {min }}=0.20$. 


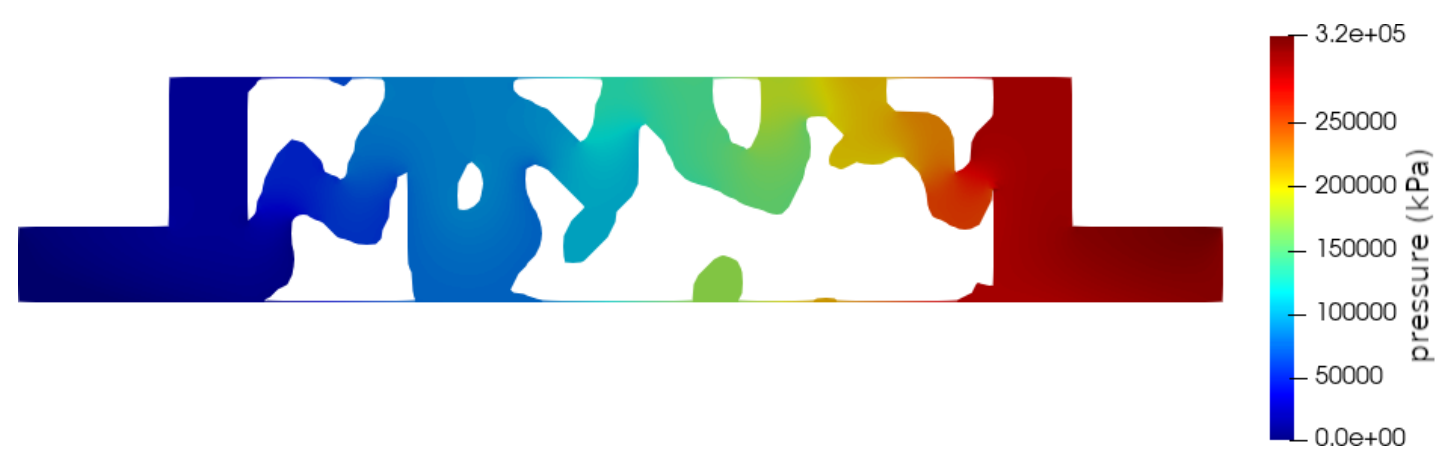

Figure 98: Pressure field of modified design domain with $\mathrm{kmax}=10^{10}$ and $r_{\min }=0.20$.

In order to explain and explore how the labyrinth seal would look like, Figure 99 shows a $3 \mathrm{D}$ perspective of previous simulation.

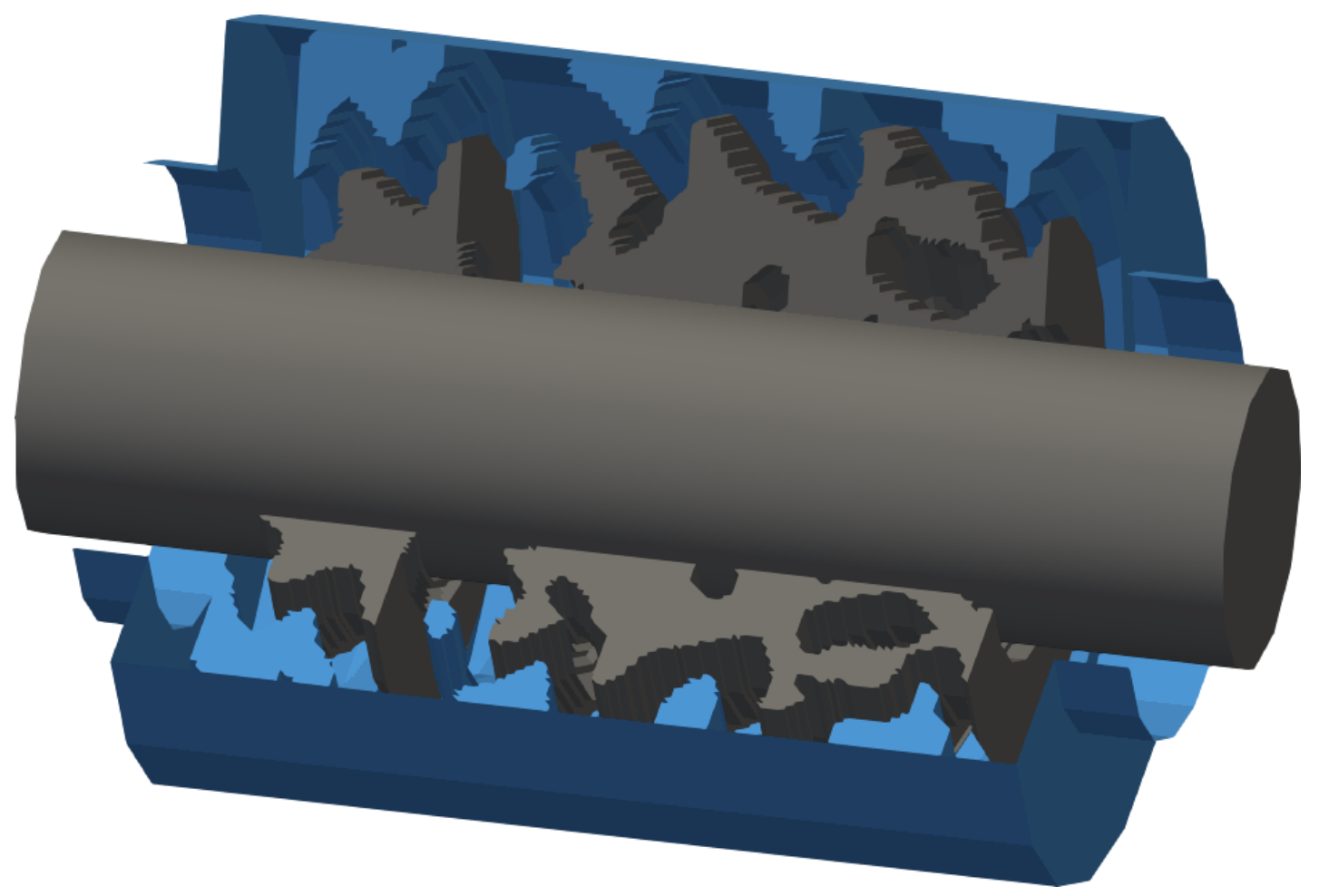

Figure 99: 3D apperance of labyrinth seal considering diodicity.

As it could be seen the diodicity are satisfatory when designing 2D swirl results and brings complex geometries that are not intuitive to a labyrinth seal, however it is still far from a reasonable solution, since it generates lots of solution with floating islands. Besides, $W_{F} F$ term is not enough to adjust a solution to clear the gap flow with satisfatory diodicity efficiency.

All diodicity values of previous simulation are shown in Table 7 , however it can be seen that in a $2 \mathrm{D}$ swirl system, the diodicity values are near 1.0, because circumferential 
velocity plays an important role on the computation of pressure and diodicity.

Table 7: Diodicity values in 2D swirl system.

\begin{tabular}{cc} 
Labyrinth seal & $D i$ \\
\hline Figure 73 & 1.01 \\
Figure 78 & 1.0 \\
Figure 83 & 1.02 \\
Figure 87 & 1.04 \\
Figure 91 & 1.05 \\
Figure 95 & 1.01 \\
\hline
\end{tabular}

\subsubsection{D swirl fluid diodes design considering vorticity}

Diodicity brings complex optimized geometry, however the challenge of blocking entrance is not completely solved and despite changing the factor $W_{F}$ can solve the problem, the solution can also be degenerated as shown in Figure 78.

For this reason the vorticity term is added to the objective function and considering the values in Table 8, with a initial guess full of fluid in the design domain. The result can be seen in Figure 100 and plane and circumferential velocity plot in Figures 101 and 102, respectively. Pressure field is shown in Figure 103.

Table 8: Optimization parameters considering vorticity

\begin{tabular}{cc}
\hline parameters & value \\
\hline$d$ & $40 \mathrm{~mm}$ \\
$R e$ & 200 \\
$n$ & $100 \mathrm{rpm}$ \\
$r_{\min }$ & $0.20 \mathrm{~mm}$ \\
$\kappa_{\text {max }}$ & $10^{10}$ \\
$W_{F}$ & 0.1 \\
$W_{E}$ & 0 \\
$W_{V}$ & $2 \cdot 10^{-7}$ \\
$V_{\text {up }}$ & $80 \%$ \\
$V_{\text {down }}$ & $50 \%$ \\
\hline
\end{tabular}




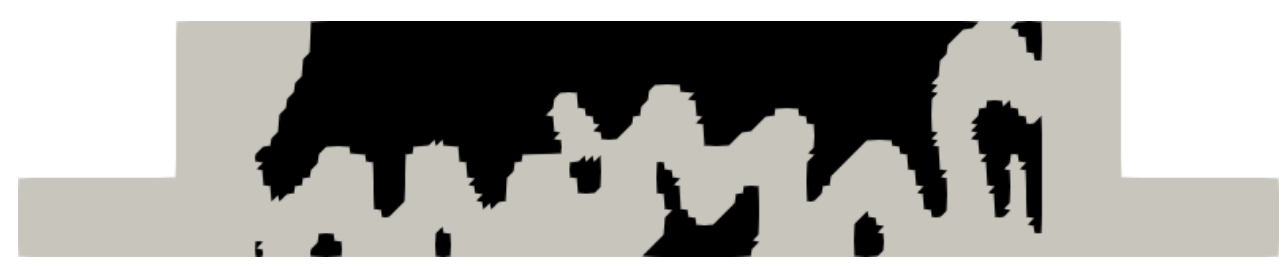

Figure 100: Vorticity with $\mathrm{kmax}=10^{10}$ and $r_{\min }=0.20$.

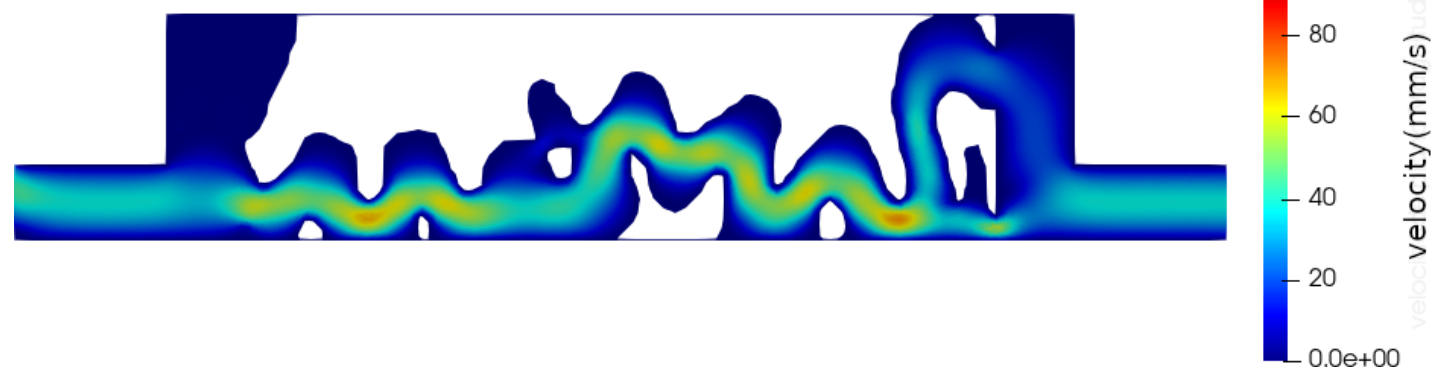

Figure 101: Modulus of plane velocity of modified design domain with vorticity.

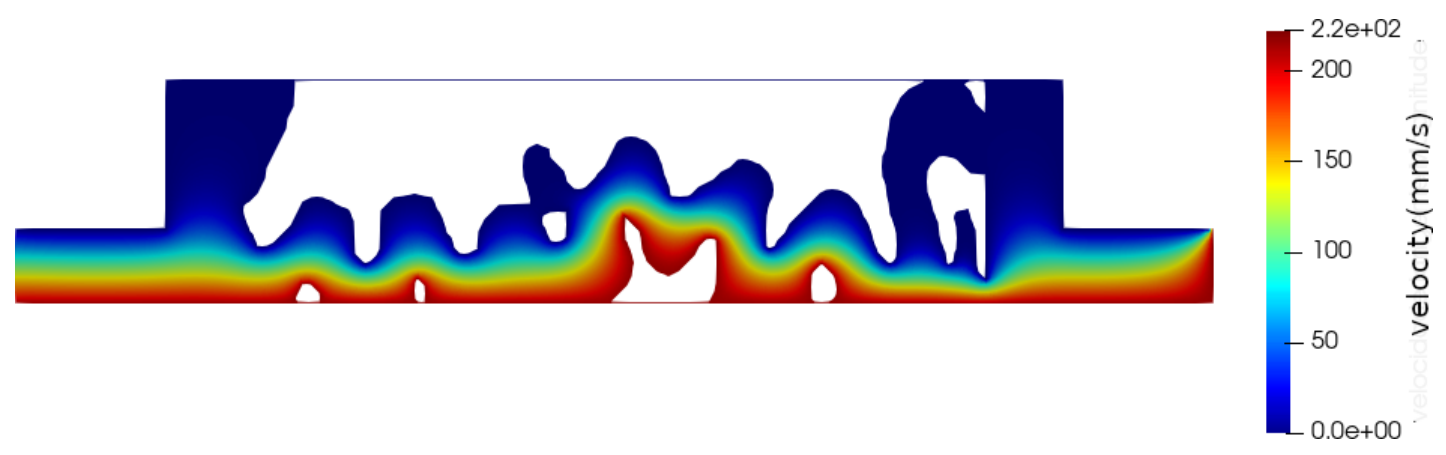

Figure 102: Circumferential velocity plot of modified design domain with vorticity.

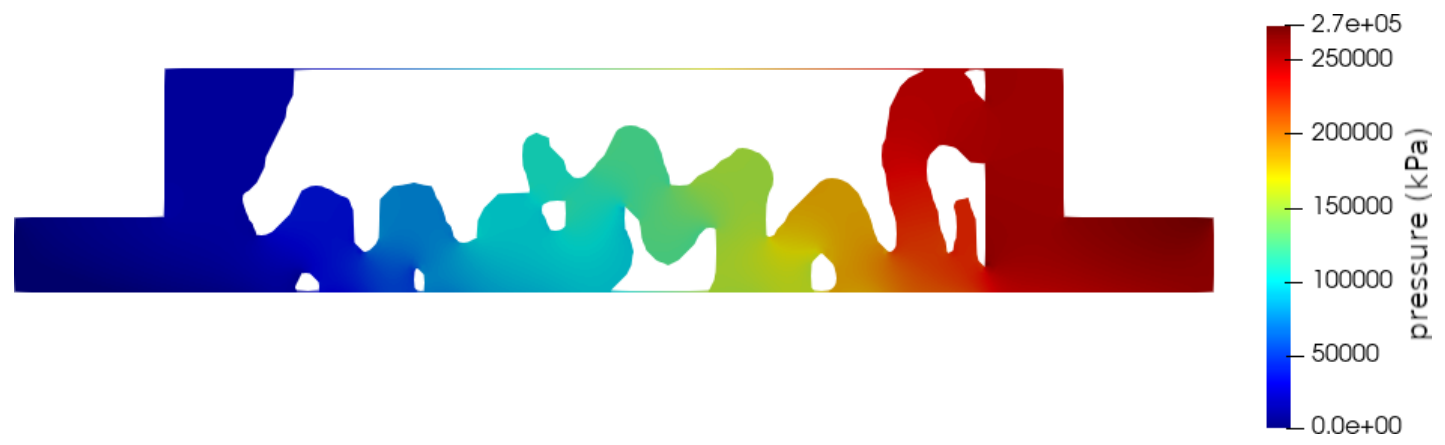

Figure 103: Pressure field of modified design domain with vorticity.

To explore shortly the influence of the $W_{V}$ factor in the objective function (Equation 3.11 ), it is raised to $2 \cdot 10^{-6}$ and the result is shown in Figure 104 with plane and cir- 
cumferential velocity plot in Figures 105 and 105, respectively. Pressure field is shown in Figure 107.

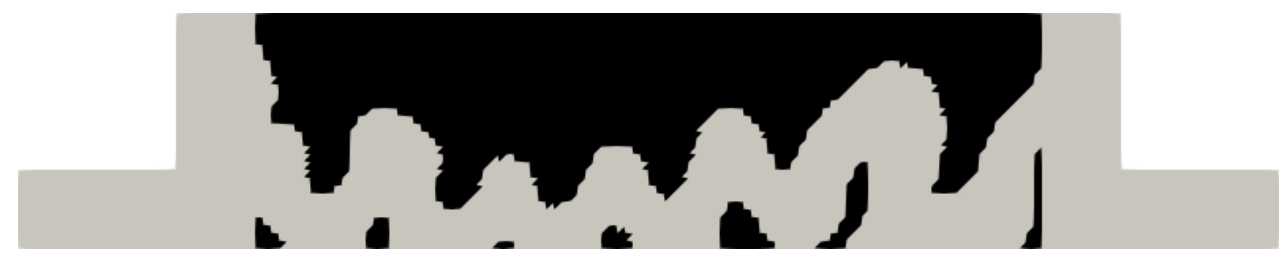

Figure 104: $W_{V}$ raised to $2 \cdot 10^{-6}$.

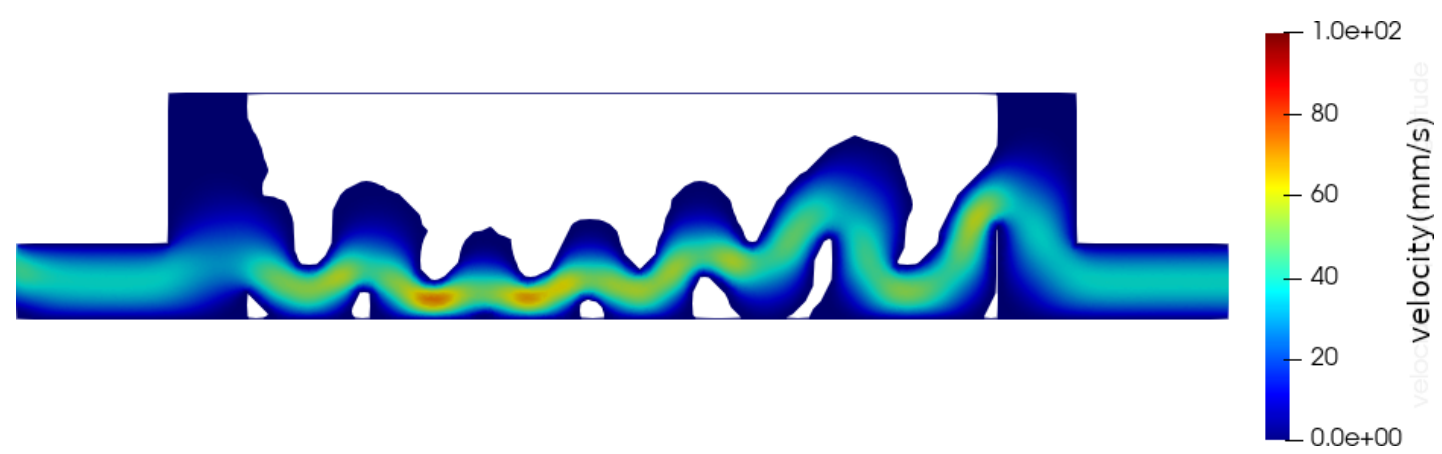

Figure 105: Modulus of plane velocity with $W_{V}=2 \cdot 10^{-6}$.

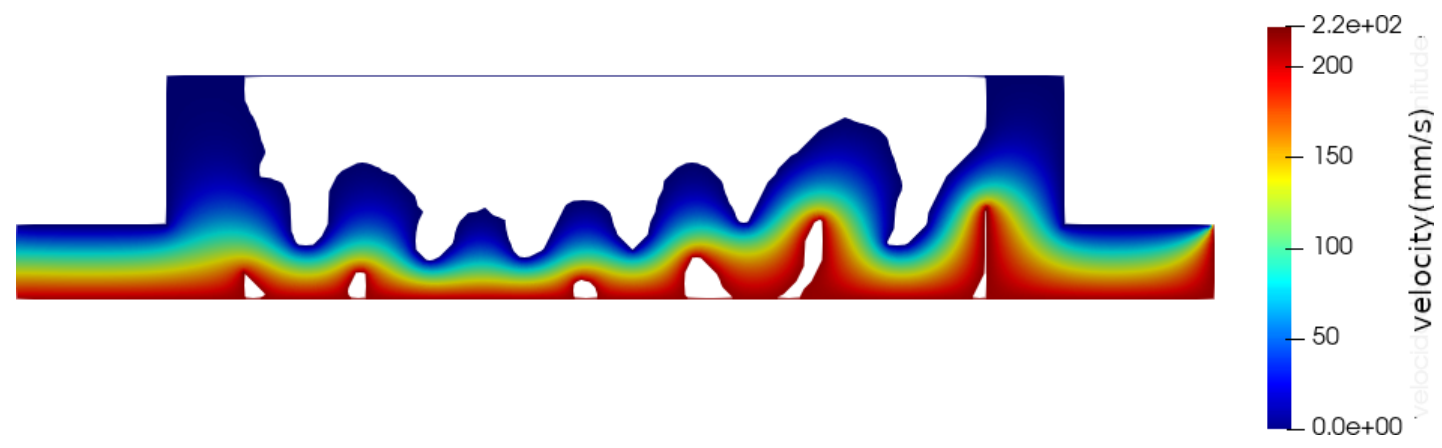

Figure 106: Circumferential velocity plot with $W_{V}=2 \cdot 10^{-6}$.

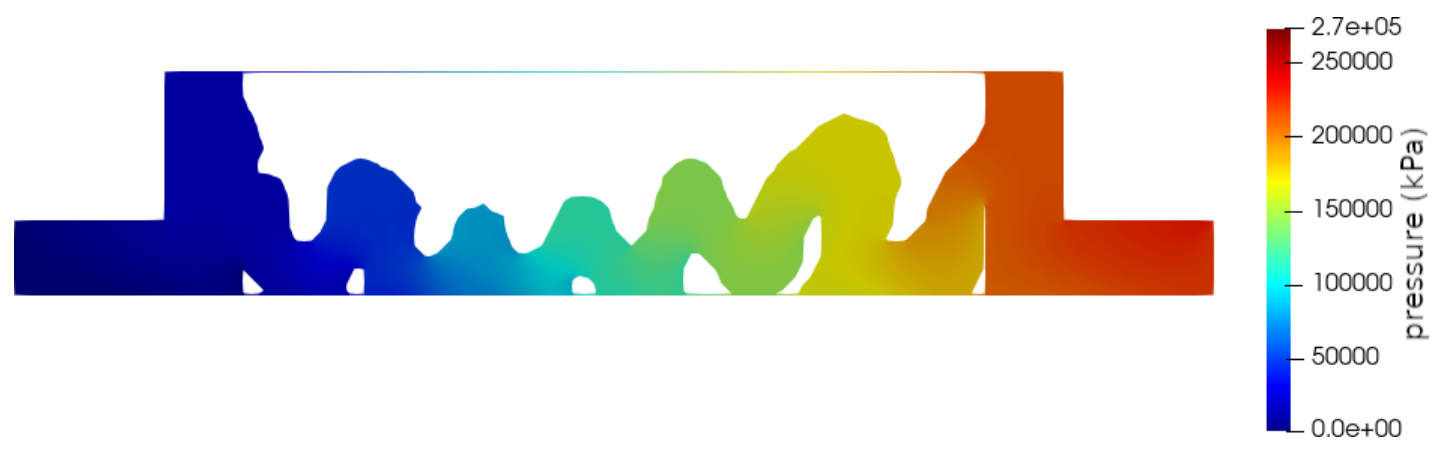

Figure 107: Pressure field with $W_{V}=2 \cdot 10^{-6}$. 
The repeated found pattern, which looks like a spring,is very interesting to the problem, since it creates a tortous path, which is the main idea of a labyrinth seal. This kind of repeated pattern is not commonly obtained by topology optimization problems, however the proposed method has succeeded.

Besides, it raises the question if the restricted design domain would be necessary at this point. Figure 108 shows that full domain can also be optimized, however for the sake of finding reasonable solution, from this point on, the design domain is kept with the gap. Plane and circumferential velocity plot are shown in Figure 109 and 110, respectively. Pressure field is shown in Figure 111.

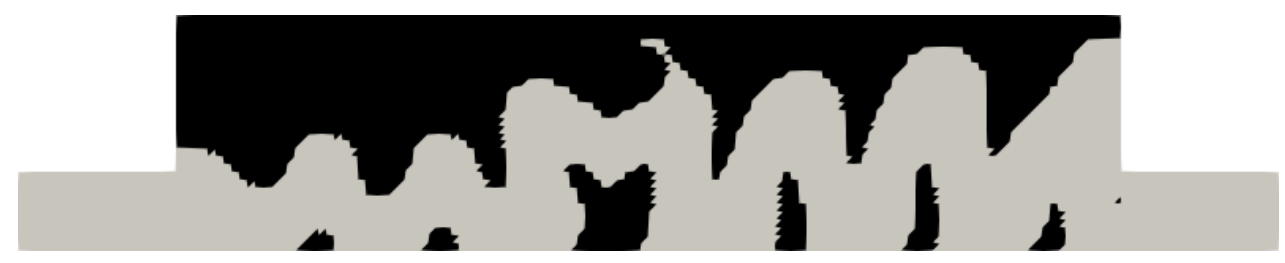

Figure 108: Full domain with $W_{V}=2 \cdot 10^{-6}$.

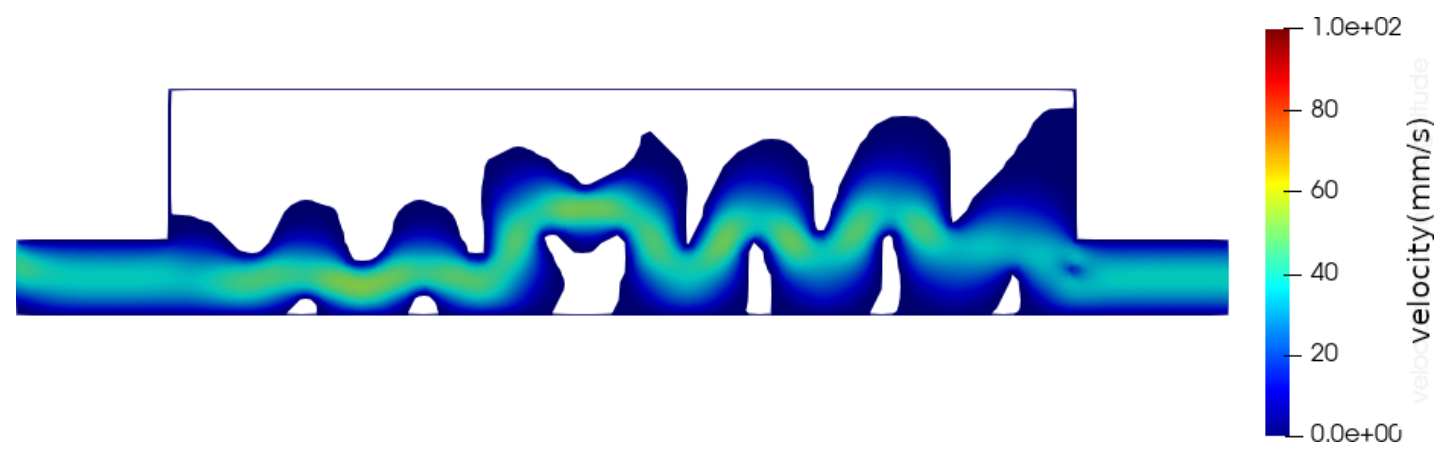

Figure 109: Modulus of plane velocity of full domain with $W_{V}=2 \cdot 10^{-6}$.

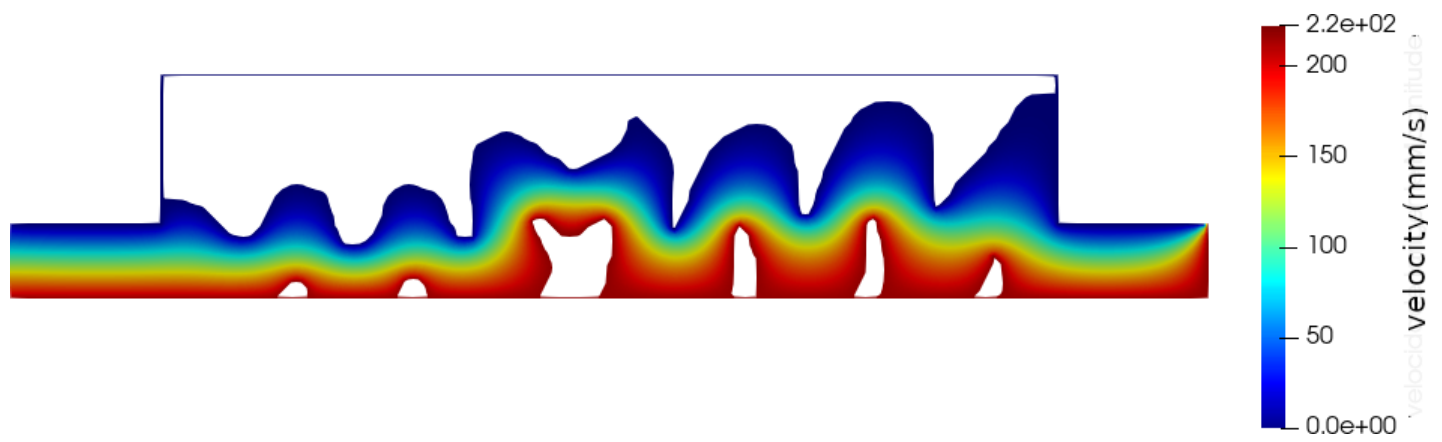

Figure 110: Circumferential velocity of full domain with $W_{V}=2 \cdot 10^{-6}$. 


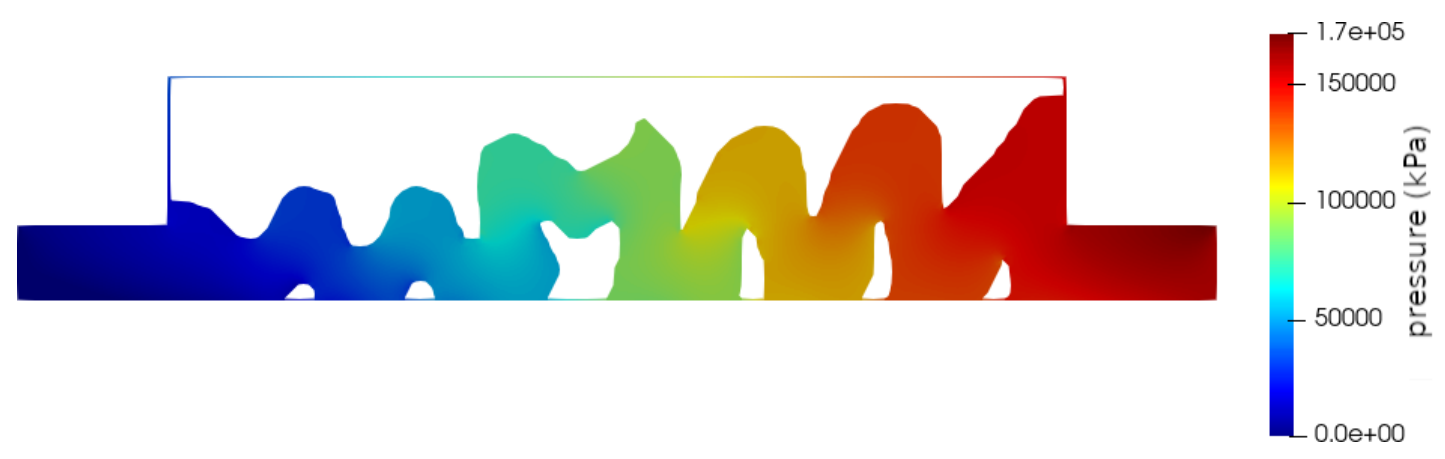

Figure 111: Pressure field of full domain with $W_{V}=2 \cdot 10^{-6}$.

From this point on, the performance of labyrinth seals is measured based on its fluid impedance $(\sqrt{\Delta p} / Q)$, which is related to pressure difference and it is the inverse of the constant of valves usually called as $K_{v}$ found in manufactures' handbooks. This is usefull to enable comparison with valves on the market.

Table 9: Performance of optimized labyrinth seal including vorticity terms (units:SI).

\begin{tabular}{cc} 
Labyrinth seal & $\sqrt{\Delta p} / Q \times 10^{5}$ \\
\hline Figure 100 & 6.33 \\
Figure 104 & 5.86 \\
Figure 108 & 4.96 \\
\hline
\end{tabular}

In order to explain and explore how the labyrinth seal would look like, Figure 112 shows a $3 \mathrm{D}$ perspective of previous simulation. 


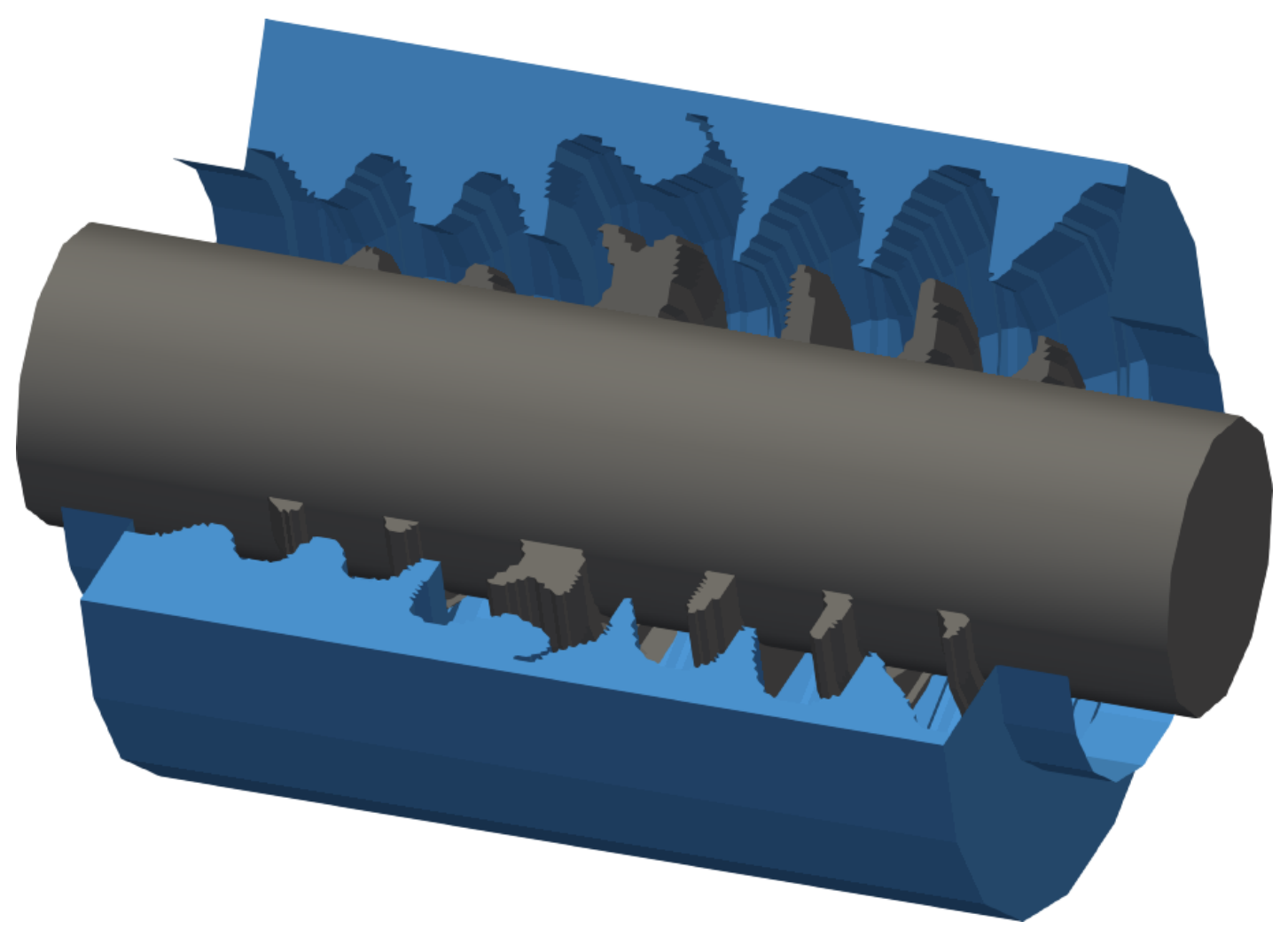

Figure 112: 3D apperance of labyrinth seal considering vorticity.

\subsection{Complete optimization problem considering Fluid- Structure interaction in fluid diodes}

Previous results is almost enough for implementing a satisfatory method for designing labyrinth seals, however, sometimes small islands can appear without any support from upper or lower parts, just like Figure 100. The analysis in this section proposes a method able to eliminate floating islands from the design domain, in other words, the design variables that corresponds to solid must be attached either to stator or to shaft.

From this section on, the model is complete with all objective function terms with initial guess full of fluid in the design domain, and for this reason, the next results of optimized labyrinth seal are compared to a similar called baseline with same fluid volume. It is defined in Figure 113 and it is created to compare with a common interlaced labyrinth seal, $V_{0}$ is the volume constraint of each case and $d_{0}$ is a value defined based on $V_{0}$ choice. 


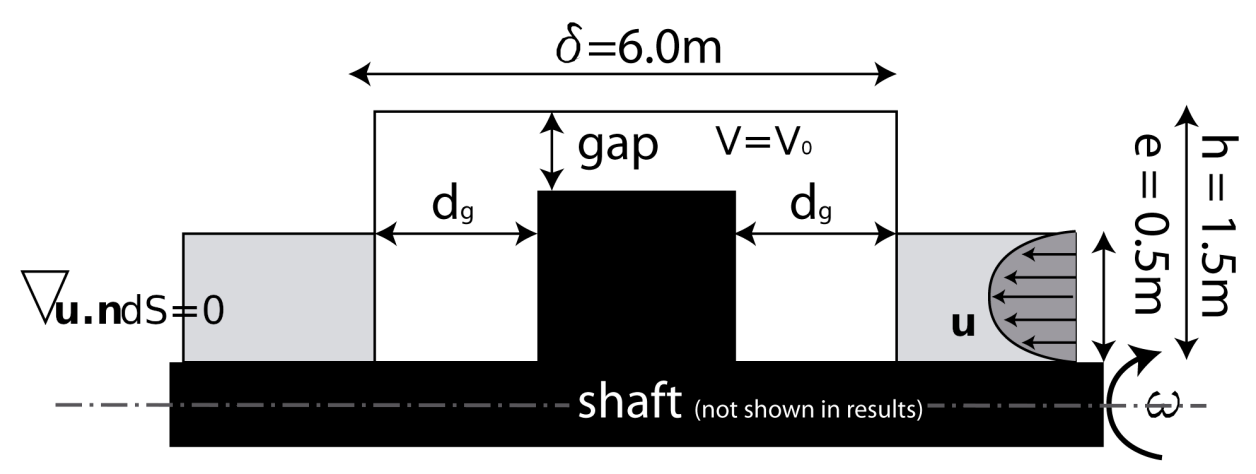

Figure 113: Baseline for labyrinth seals comparison, respecting the same volume constraint.

As boundary conditions, Figure 114 illustrates that it is used a prescribed inlet velocity, a stress free condition on outlet and a selective rotation condition depending on the position of the solid. The velocity field on inlet in this work considers a parabolic velocity profile with a corresponding Reynolds Number defined in Equation 5.3 used for all numerical examples. All parameter values are listed in Table 10.

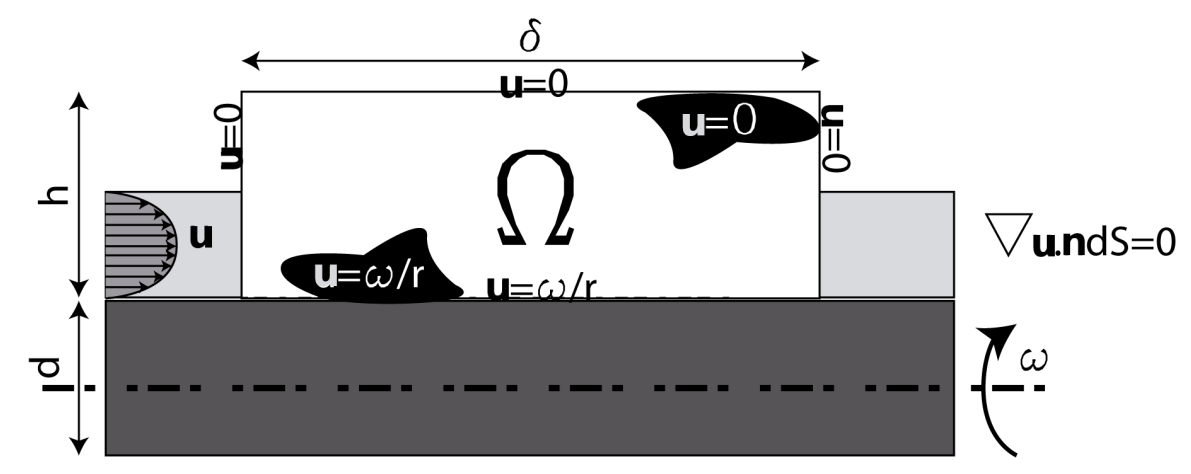

Figure 114: Boundary conditions of a rotating labyrinth seal.

$$
R e=\frac{\rho u_{a v} D}{\mu}=u_{a v} D
$$

where $\rho$ is fluid density, $u_{a v}$ is magnitude of average inlet velocity, $D$ is inlet length, and $\mu$ is dynamic viscosity of fluid.

The results can be seen in Figure 115 with interesting geometry, because the undesirable floating islands has disappeared. For the purpose of understanding the labyrinth seal behaviour the plane and circumferential velocity plot is shown in Figures 116 and 117, respectively. Pressure field is shown in Figure 118.

The deformation of optimized geometry are shown in all results only to illustrate the 
analysis, since the goal of the proposed method is to eliminate floating islands. However, it opens a possibility in future to work with materials that allow high deformation values and its impact in labyrinth seal efficiency. The convergence plot is shown in Figure 119, showing high oscillation until final solution achieved. That may occur due to the number of local minima. All deformation values in the plots are increased by 5000 times to be visual. In this case, it can be seen in Figure 120 and its efficiency is greater than the traditional (see Table 11).

In this simulation, it is possible to see that a tortous path is also found, however with a non elementary solution. The solids attached to shaft increase in the middle of the domain and the edges are slightly inclined to the right in an attempt to obstruct the flow from right to left.

Table 10: Optimization parameters in a FSI problem

\begin{tabular}{cc}
\hline parameters & value \\
\cline { 2 - 2 }$d$ & $40 \mathrm{~mm}$ \\
$R e$ & 200 \\
$n$ & $100 \mathrm{rpm}$ \\
$r_{\text {min }}$ & $0.20 \mathrm{~mm}$ \\
$\kappa_{\text {max }}$ & $10^{10}$ \\
$W_{F}$ & 0.1 \\
$W_{E}$ & $10^{-20}$ \\
$W_{V}$ & $2 \cdot 10^{-7}$ \\
$V_{\text {up }}$ & $80 \%$ \\
$V_{\text {down }}$ & $50 \%$ \\
\hline
\end{tabular}

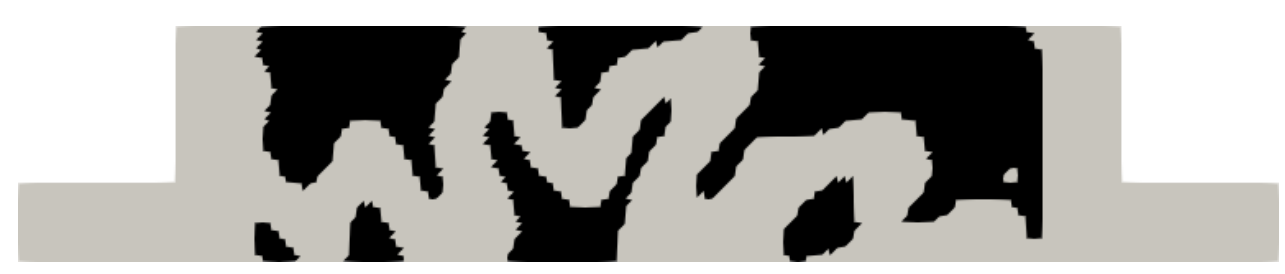

Figure 115: Labyrinth seal using fluid structure interaction algorithm. 


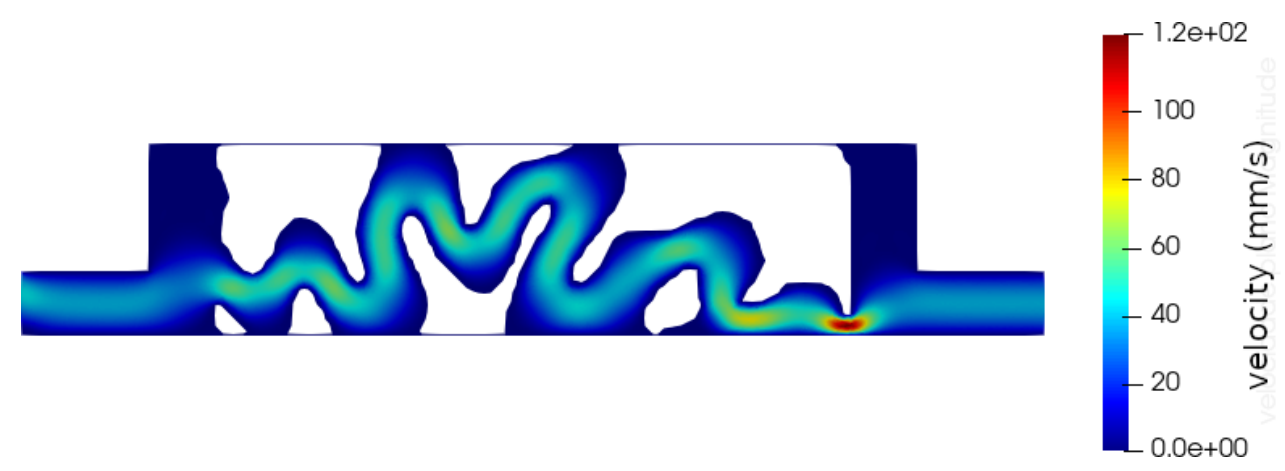

Figure 116: Modulus of plane velocity in the Labyrinth seal using fluid structure interaction algorithm.

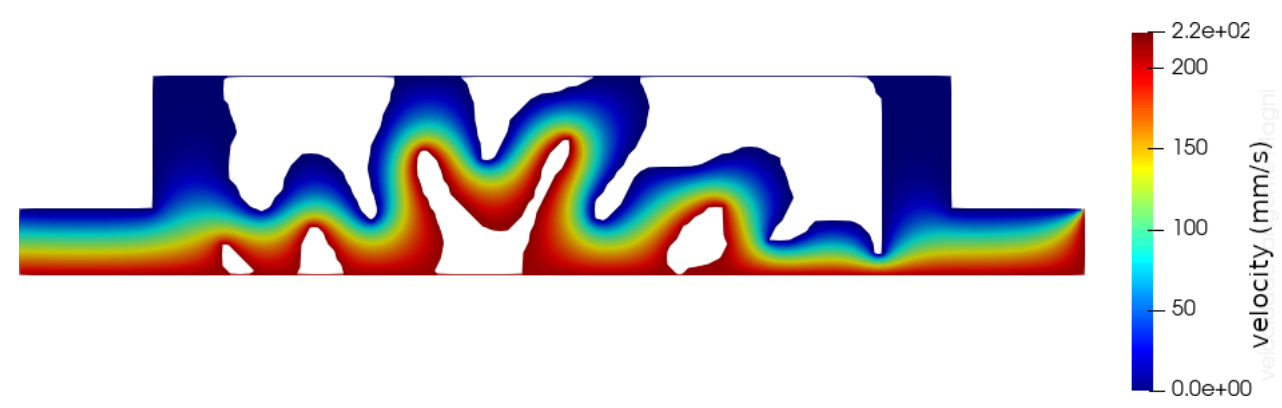

Figure 117: Circumferential velocity plot in the Labyrinth seal using fluid structure interaction algorithm.

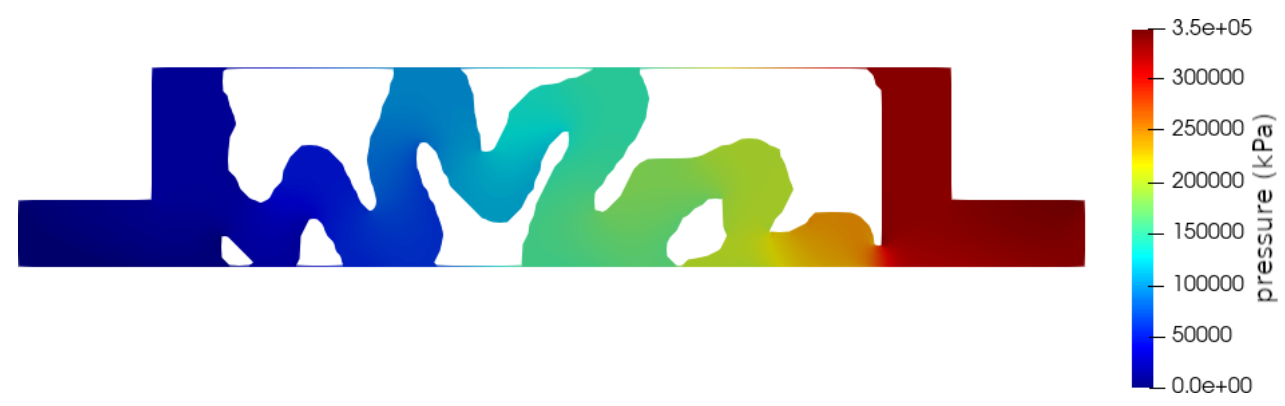

Figure 118: Pressure field in the Labyrinth seal using fluid structure interaction algorithm. 


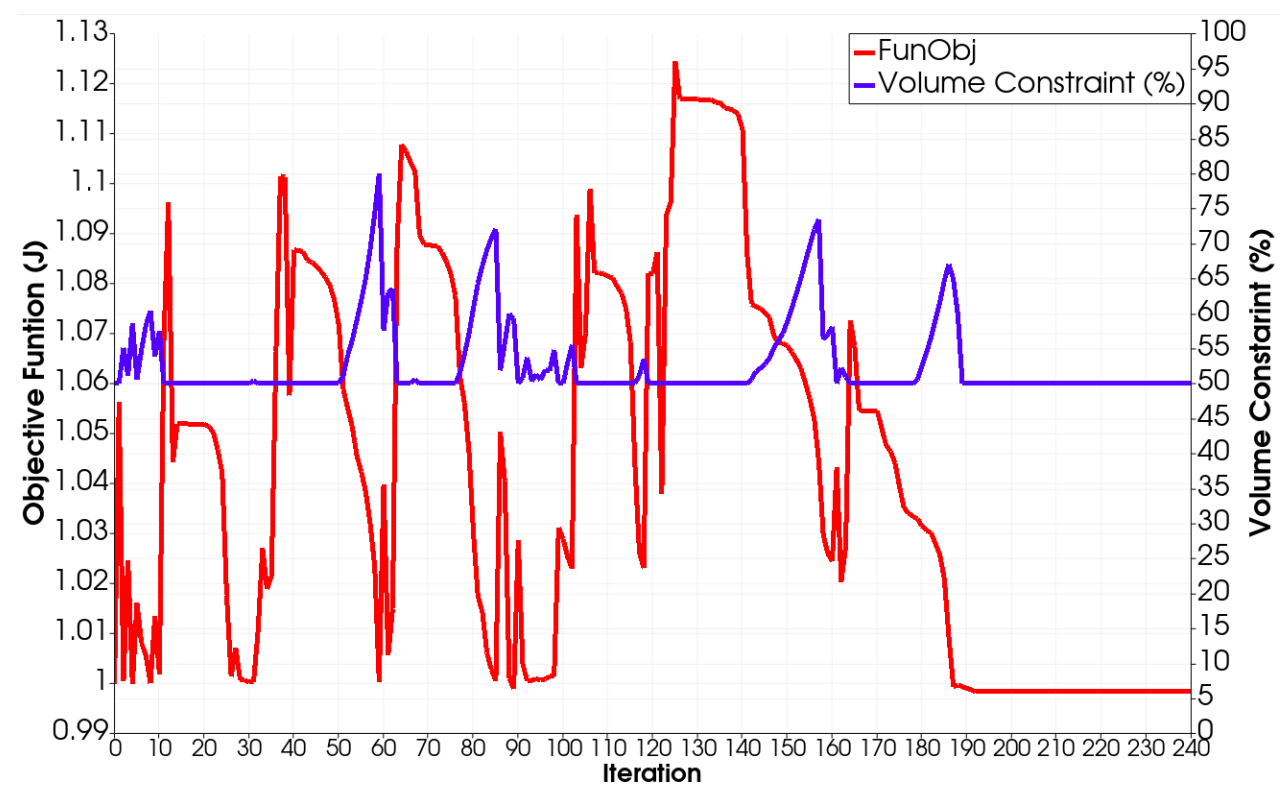

Figure 119: Objective Function in a full optimization problem considering FSI.

Table 11: Performance of optimized labyrinth seal using FSI (units:SI).

\begin{tabular}{cc}
\hline Labyrinth seal & $\sqrt{\Delta p} / Q \times 10^{4}$ \\
\hline Baseline & 1.15 \\
Optimized & 2.28 \\
\hline
\end{tabular}

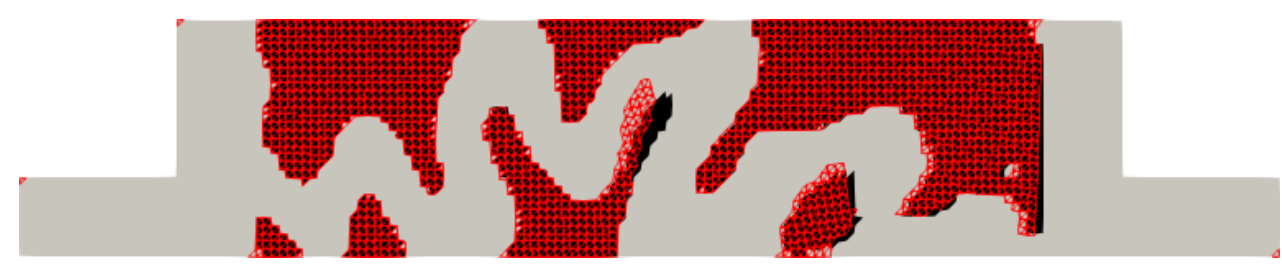

Figure 120: Displacement in red due to flow in Labyrinth seal.

In order to explain and explore how the labyrinth seal would look like, Figure 121 shows a $3 \mathrm{~d}$ perspective of previous simulation. 


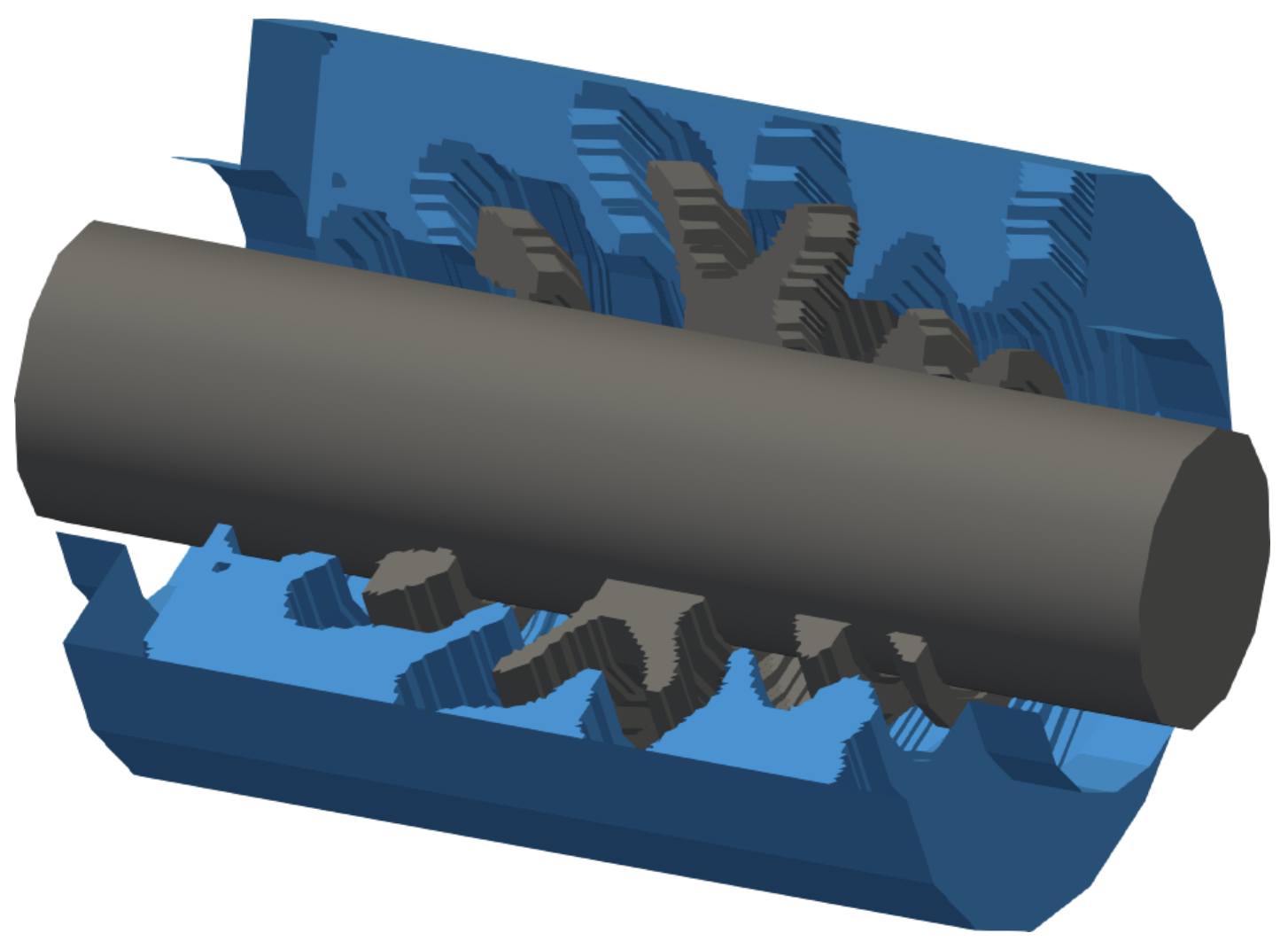

Figure 121: 3D apperance of labyrinth seal of a full optimization problem.

\subsubsection{Aspect ratio influence}

The problem studied in this work has several local minima, thus it is complicated to compare different aspect ratio. However, this section shows that a large labyrinth seal can also be modeled as a sequential of small optimized unit cells.

The solution found for different unit cell geometries can be seen in Figure 122 and how this could be implemented in Figure 123. The advantage of this method would be low computational cost due to the low number of design variables.

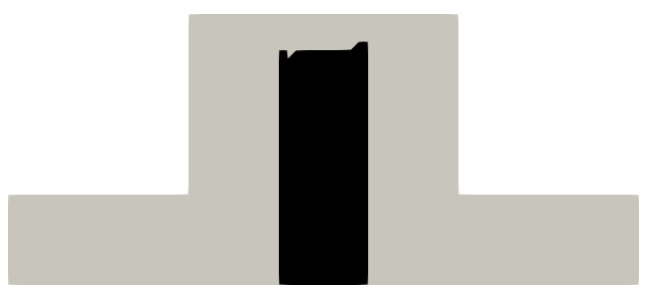

(a) Aspect ratio of 1:1

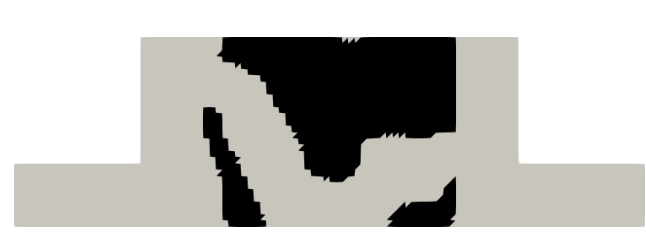

(b) Aspect ratio of 2:1

Figure 122: Aspect ratio influence 


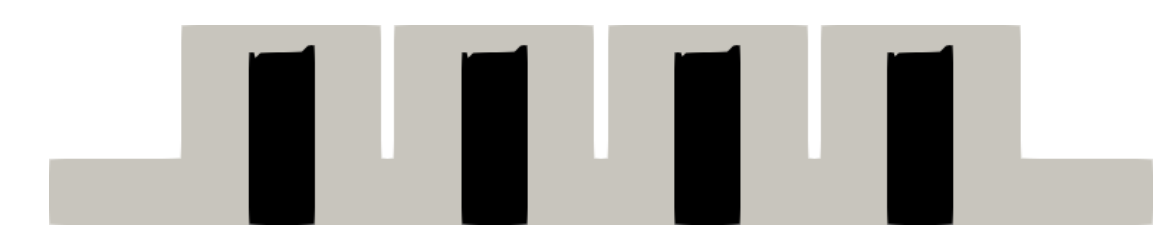

(a) Aspect ratio of 1:1

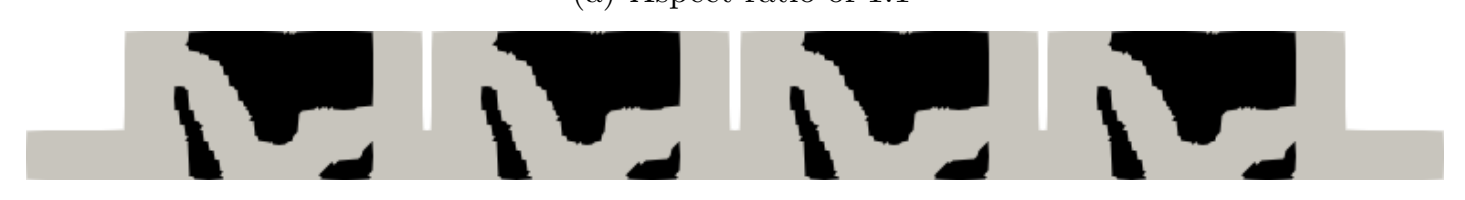

(b) Aspect ratio of 2:1

Figure 123: Aspect ratio influence

Figures 124 and 125 show the plane and perpendicular velocity plot, respectively, Figure 126 shows the pressure field of found results and Table 12 shows the disadvantages of using such aspect ratios, showing the proximity with the baseline efficiency. To illustrate how the geometry would suffer deformation from the fluid flow, it is shown in Figure 127. The smaller aspect ratio seal has much more more deformation in the same scale, thus if the designer is not worried with deformation and does not want complex geometry, it may be more beneficial to choose it.

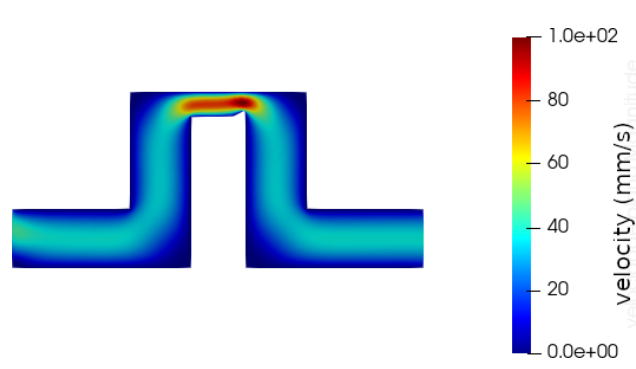

(a) Aspect ratio of 1:1

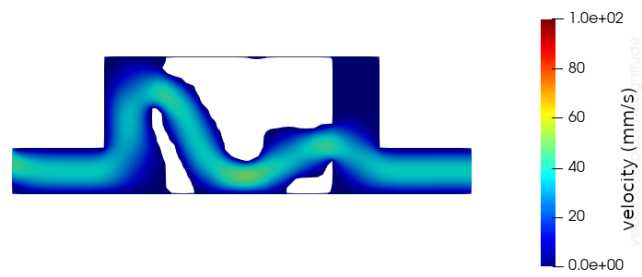

(b) Aspect ratio of 2:1

Figure 124: Modulus of plane velocity of unit cell for different aspect ratio.

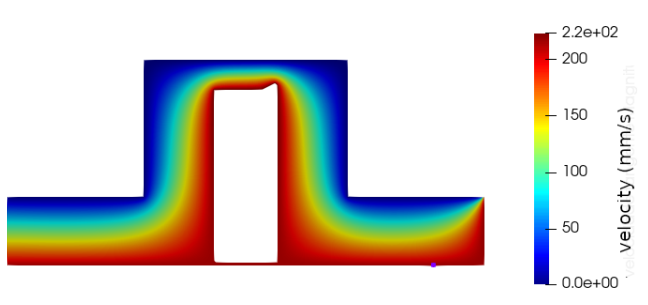

(a) Aspect ratio of 1:1

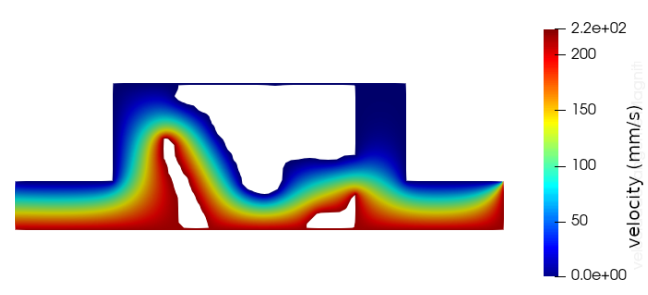

(b) Aspect ratio of 2:1

Figure 125: Circumferential velocity plot of unit cell for different aspect ratio. 


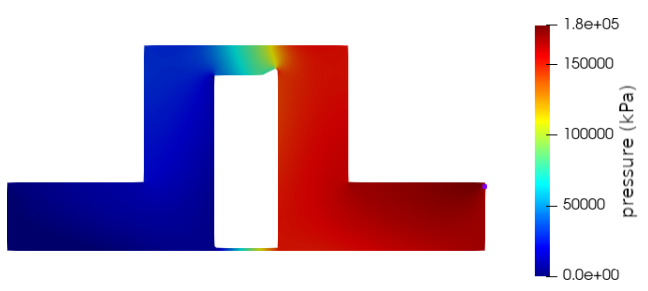

(a) Aspect ratio of 1:1

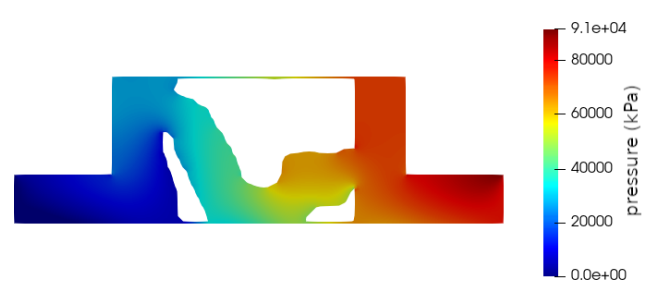

(b) Aspect ratio of 2:1

Figure 126: Pressure field of unit cell for different aspect ratio.

Table 12: Performance of optimized labyrinth seal using FSI with different aspect ratio considering only one cell.

\begin{tabular}{cc}
\hline Labyrinth seal & $\sqrt{\Delta p} / Q \times 10^{4}$ \\
\hline Baseline aspect ratio 1:1 & 1.60 \\
Optimized aspect ratio 1:1 & 1.60 \\
\hline Baseline aspect ratio 2:1 & 1.11 \\
Optimized aspect ratio 2:1 & 1.12 \\
\hline
\end{tabular}

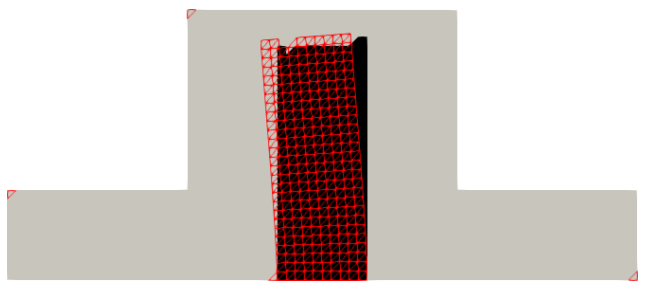

(a) Aspect ratio of 1:1

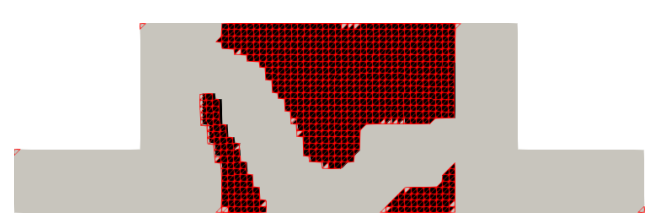

(b) Aspect ratio of 2:1

Figure 127: Deformation of geometries from different aspect ratio influence

\subsubsection{Diameter influence}

This section studies how it should be the change in the optimized geometry for a labyrinth seal with different shaft diameters.

The results of the proposed method with different diameter values shows that in smaller shaft diameter (Figure 128a), the solution tends to increase the size of solid parts attached to the shaft. On the other hand, Figure 128b shows a different type of solution, with a simpler tortous path solution.

The plane and circumferential velocity plots can be found in Figures 129 and 130, respectively, with efficiency values in Table 13. Pressure field is shown in Figure 131. 
The deformation (see Figure 132) shows that the result of high diameter values achieves smaller deformation in the analysis.

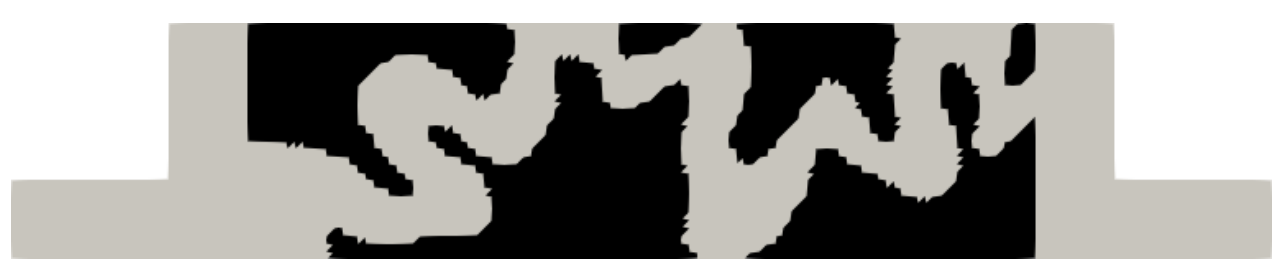

(a) Diameter of $20 \mathrm{~mm}$

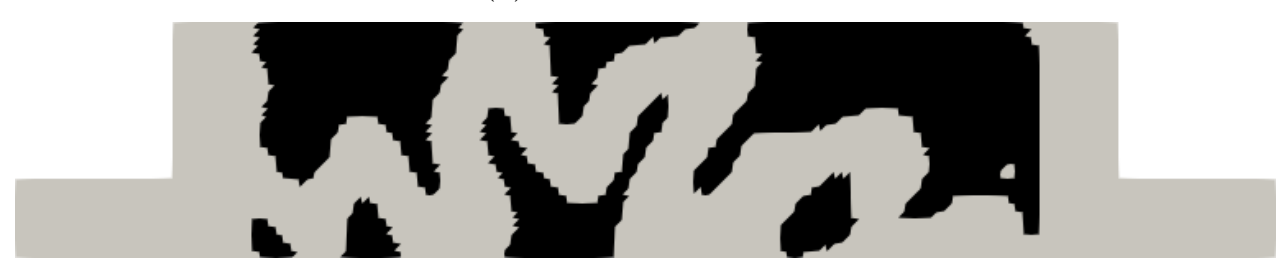

(b) Diameter of $40 \mathrm{~mm}$

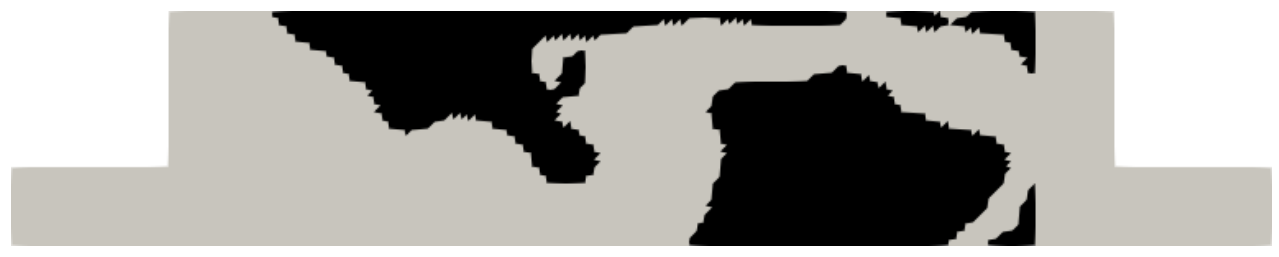

(c) Diameter of $60 \mathrm{~mm}$

Figure 128: Topology optimization results for different shaft diameters. 


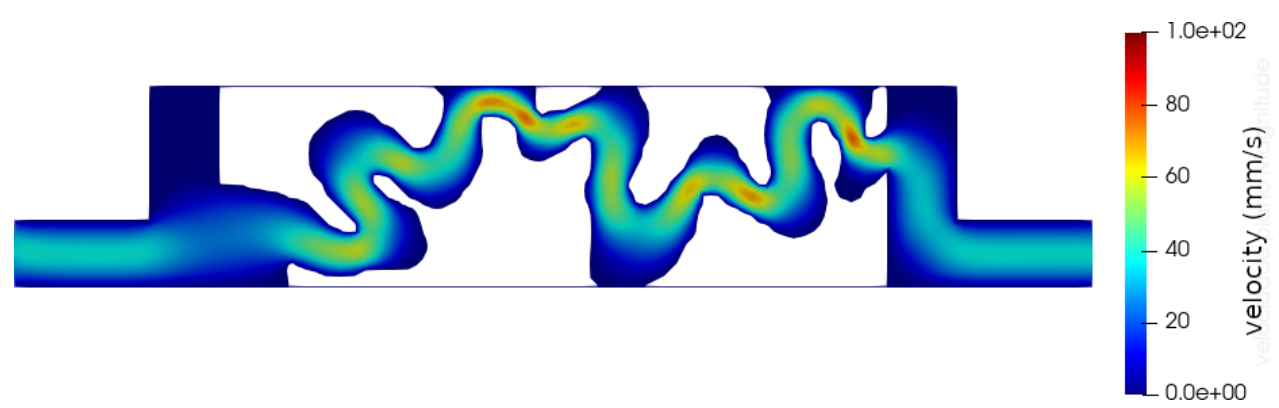

(a) Diameter of $20 \mathrm{~mm}$

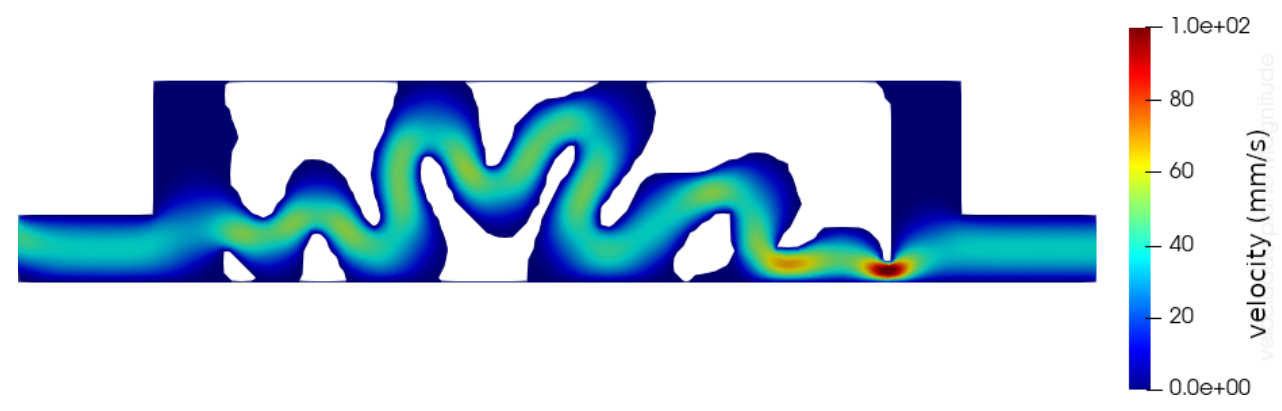

(b) Diameter of $40 \mathrm{~mm}$

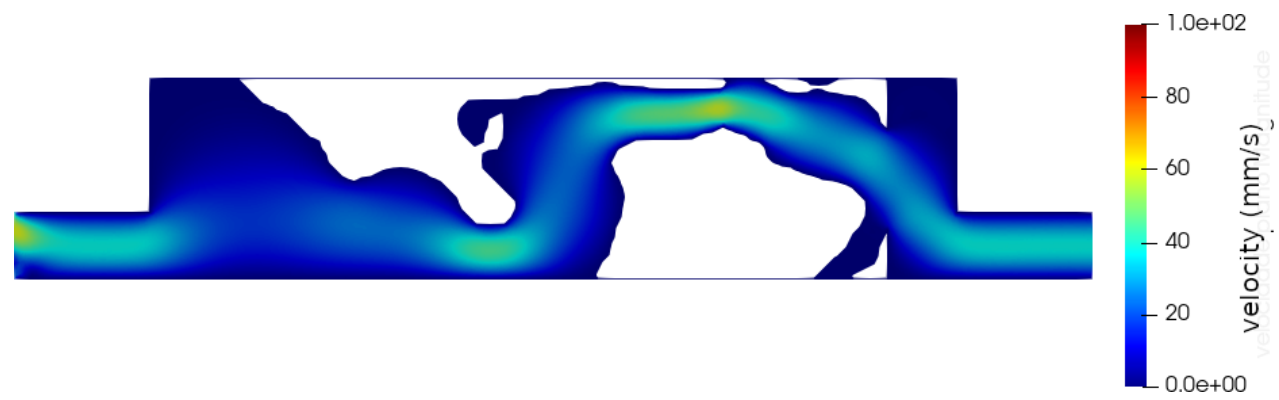

(c) Diameter of $60 \mathrm{~mm}$

Figure 129: Modulus of plane velocity for different shaft diameters. 


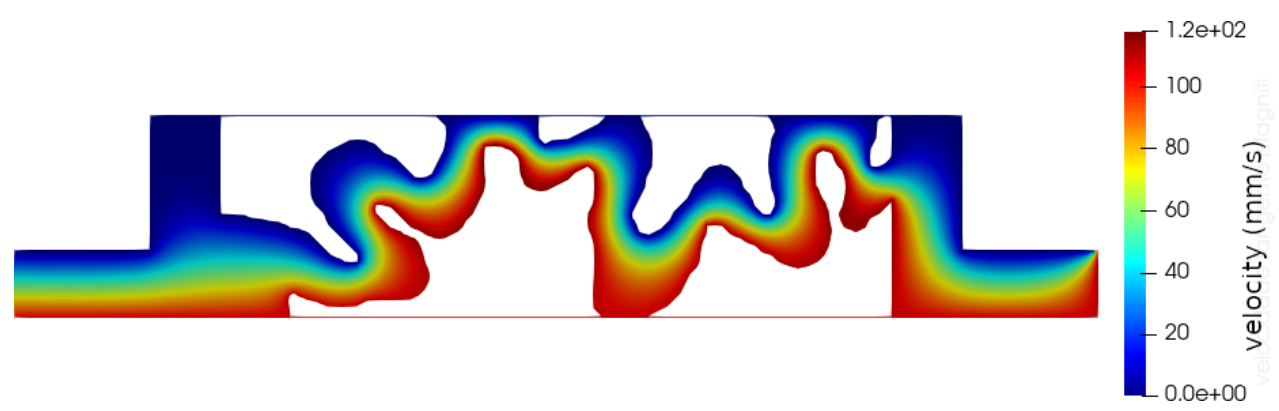

(a) Diameter of $20 \mathrm{~mm}$

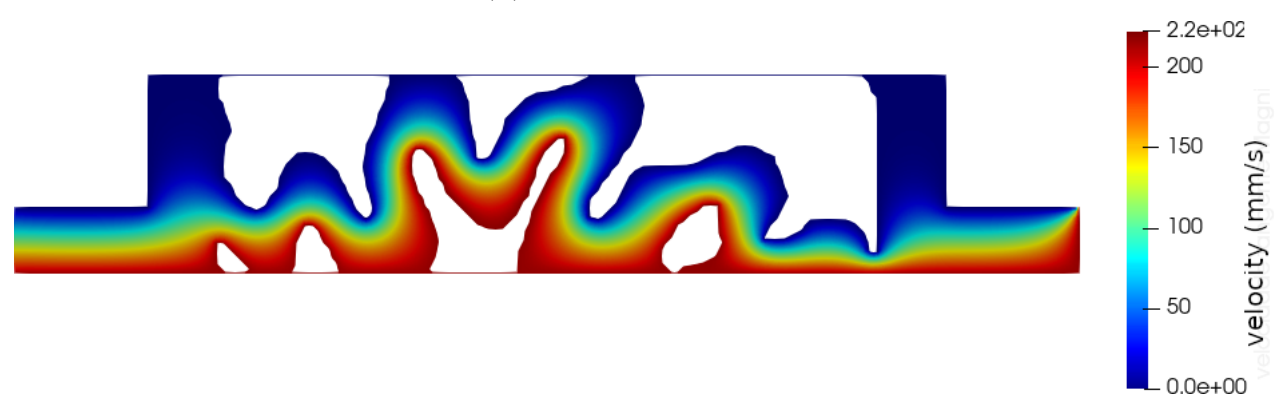

(b) Diameter of $40 \mathrm{~mm}$

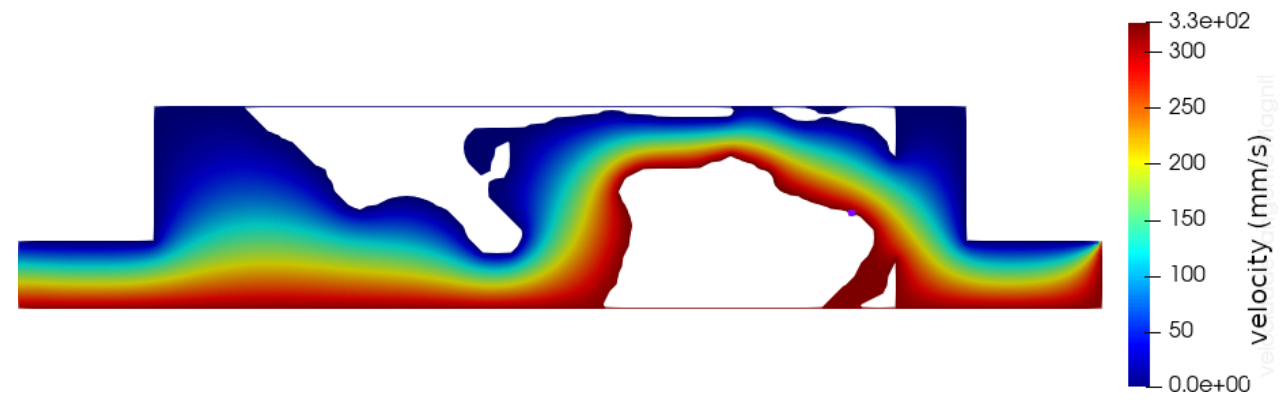

(c) Diameter of $60 \mathrm{~mm}$

Figure 130: Circumferential velocity plot for different shaft diameters. 


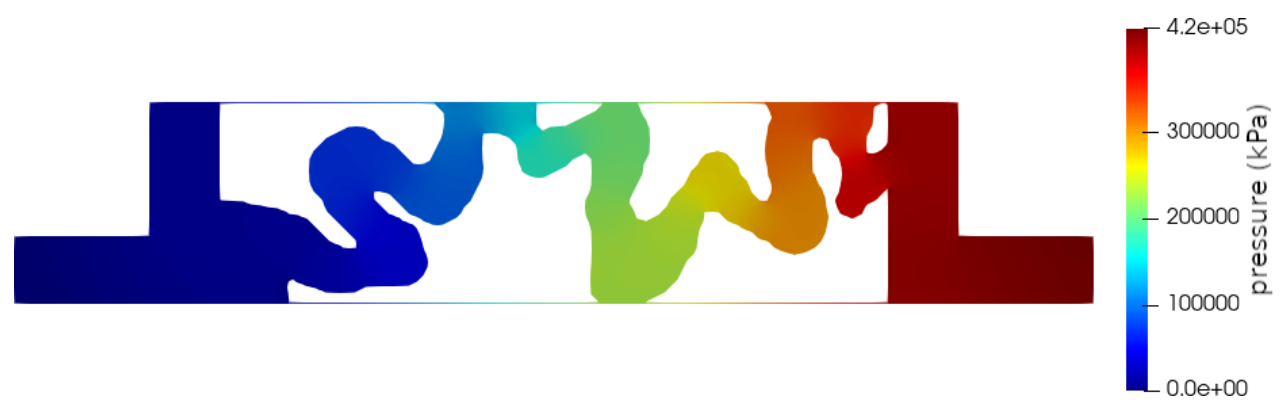

(a) Diameter of $20 \mathrm{~mm}$

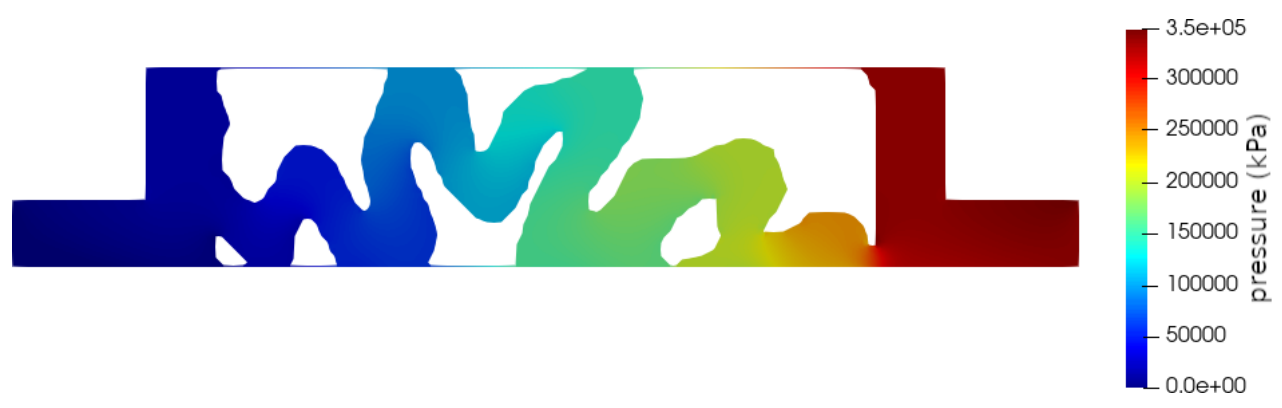

(b) Diameter of $40 \mathrm{~mm}$

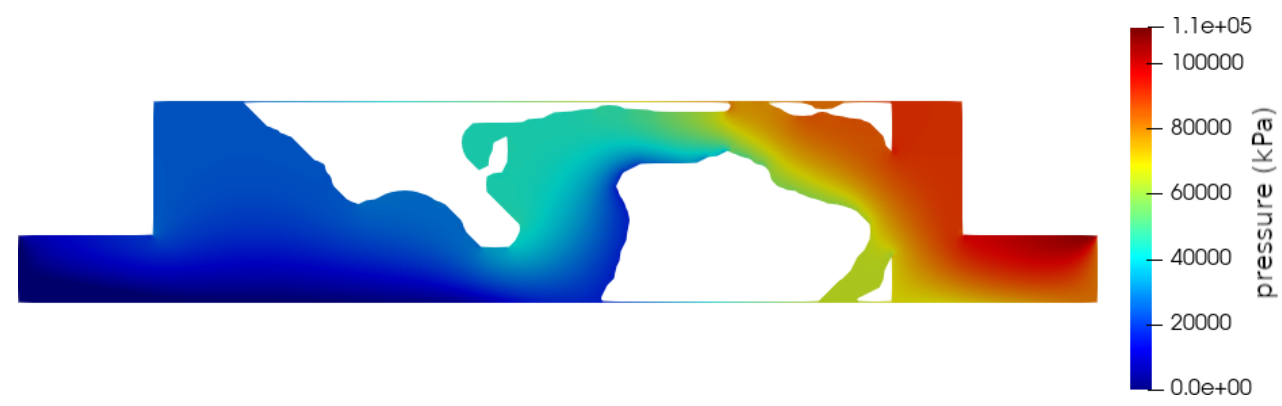

(c) Diameter of $60 \mathrm{~mm}$

Figure 131: Pressure field for different shaft diameters.

Table 13: Performance of optimized labyrinth seal using FSI with different shaft diameters.

\begin{tabular}{cc}
\hline Labyrinth seal & $\sqrt{\Delta p} / Q \times 10^{4}$ \\
\hline Baseline aspect $d=20 \mathrm{~mm}$ & 1.17 \\
Optimized aspect $d=20 \mathrm{~mm}$ & 2.54 \\
\hline Baseline aspect $d=40 \mathrm{~mm}$ & 1.15 \\
Optimized aspect $d=40 \mathrm{~mm}$ & 2.28 \\
\hline Baseline aspect $d=60 \mathrm{~mm}$ & 1.11 \\
Optimized aspect $d=60 \mathrm{~mm}$ & 1.14 \\
\hline
\end{tabular}




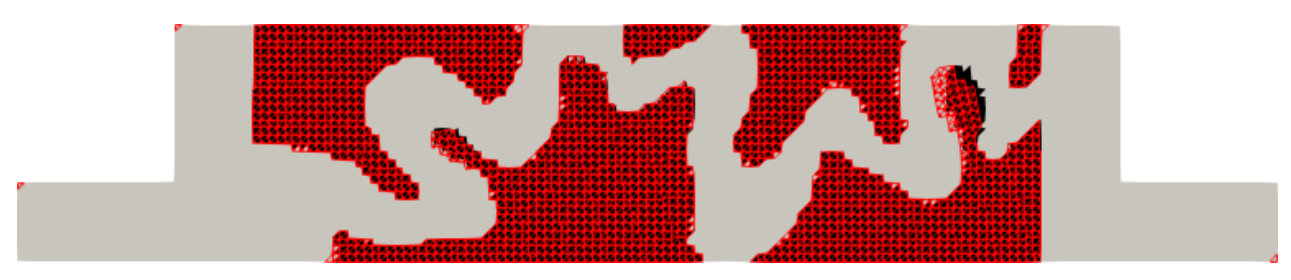

(a) Diameter of $20 \mathrm{~mm}$

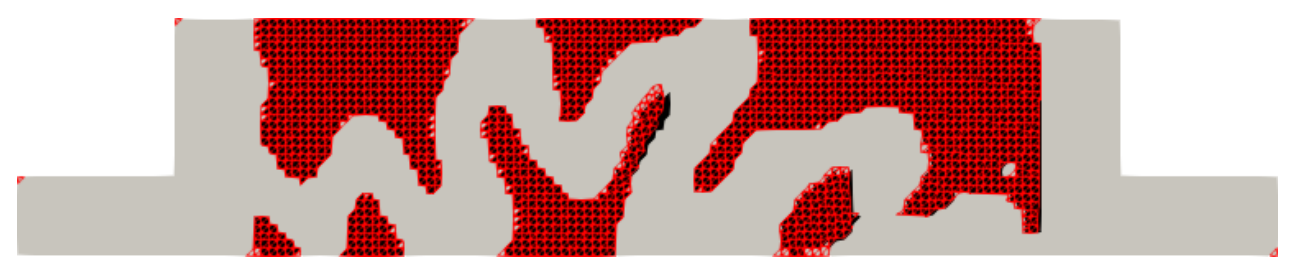

(b) Diameter of $40 \mathrm{~mm}$

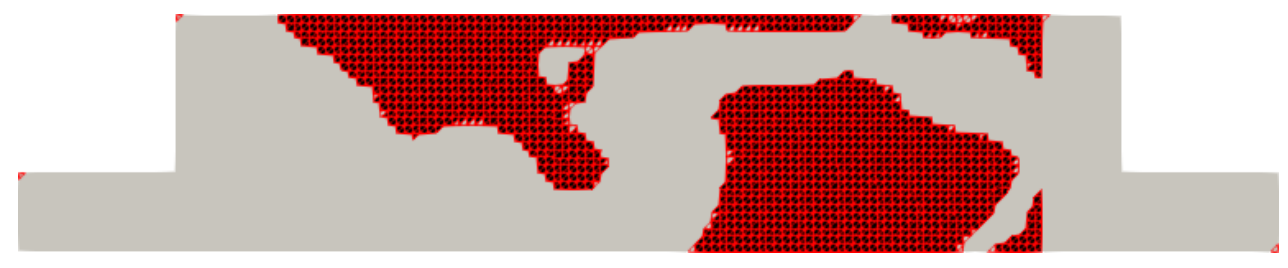

(c) Diameter of $60 \mathrm{~mm}$

Figure 132: Deformation of topology results for different shaft diameters.

\subsubsection{Rotational speed influence}

This section explores the rotational speed of the shaft in the optimized geometry for a labyrinth seal with a range of values from 50 to $300 \mathrm{rpm}$. Higher values requires stabilization techniques that are not scope of this work.

The results of the proposed method are shown in Figure 133, in which can be seen that in smaller rotational values, simpler tortous path is obtained with edges inclined to the left. On the other hand, with higher rotational values, complex tortous path is created with hollow structures.

Figure 134 and 135 show the plane and circumferential velocity plot, respectively for all cases, evidencing spots without significant velocity magnitude in the high rotational speed case. Pressure field is shown in Figure 136.

Table 14 shows the performance of each device, with results presenting better efficiency than the baseline.

The idea of previous section continues here where higher rotational velocity can cause higher displacement, eliminating small structures on the shaft, which is illustrated in Figure 137. 


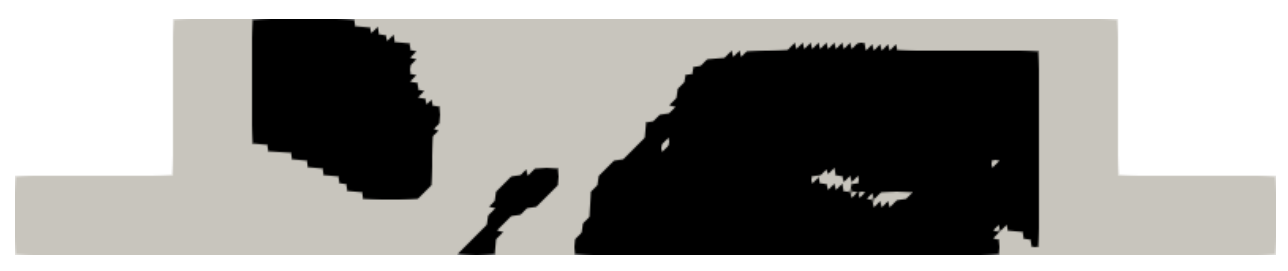

(a) Rotation of $50 \mathrm{rpm}$

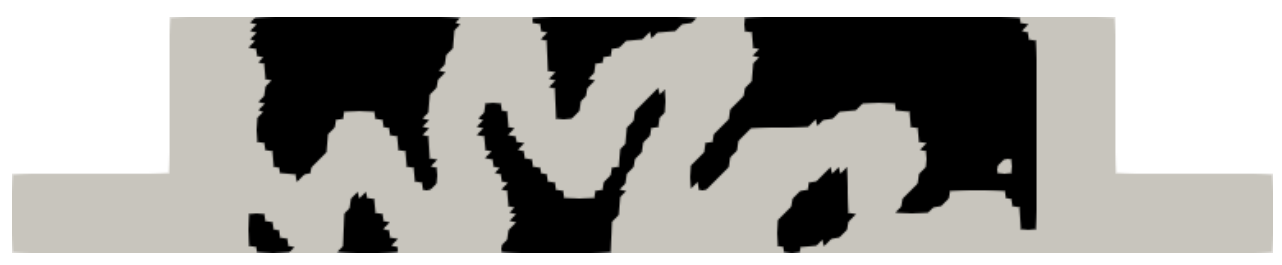

(b) Rotation of $100 \mathrm{rpm}$

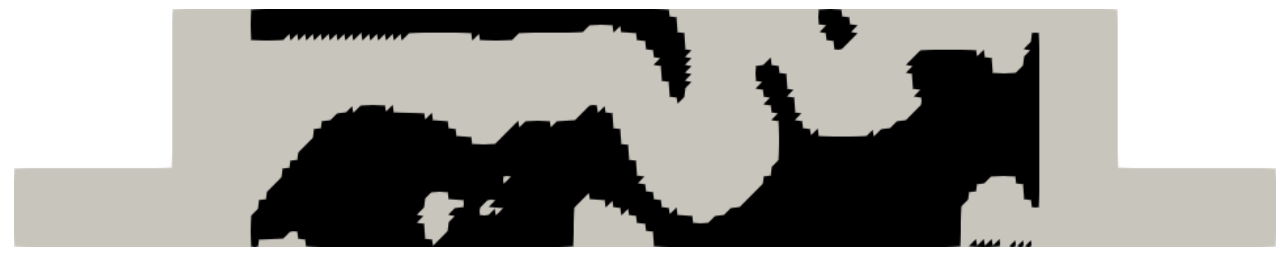

(c) Rotation of $300 \mathrm{rpm}$

Figure 133: Topology optimization results for different rotational values. 


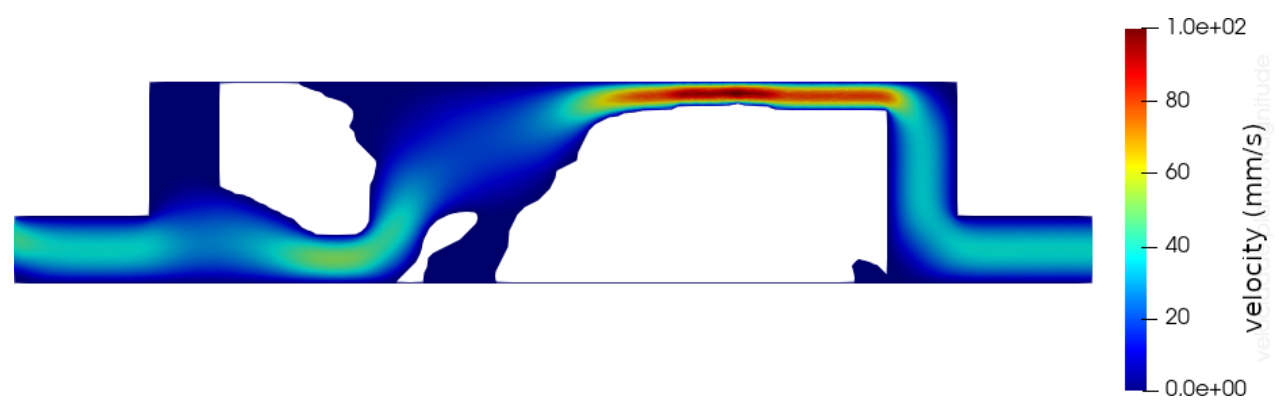

(a) Rotation of $50 \mathrm{rpm}$

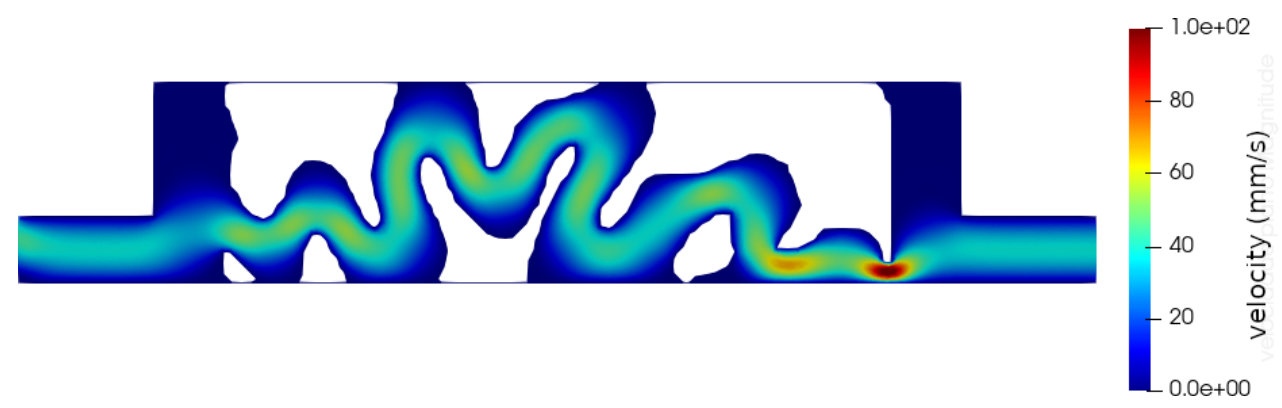

(b) Rotation of $100 \mathrm{rpm}$

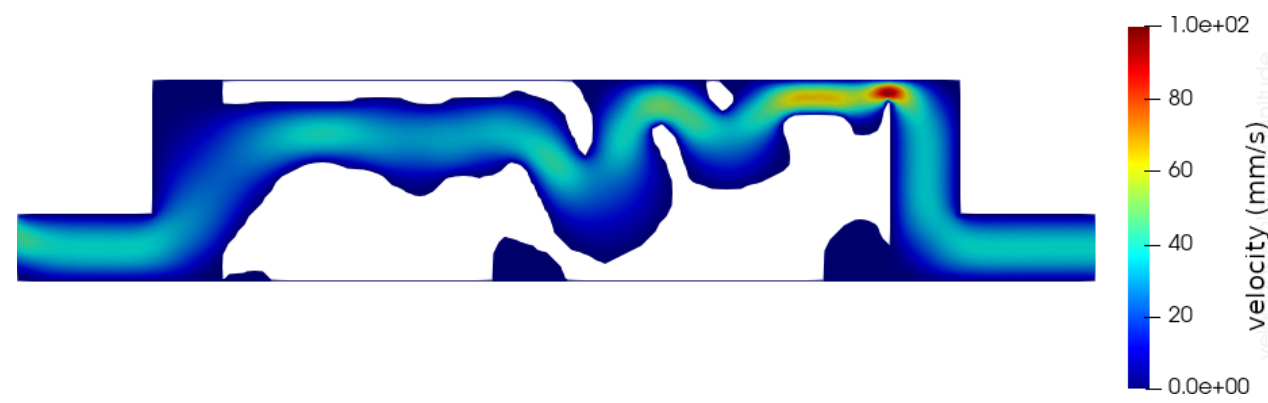

(c) Rotation of $300 \mathrm{rpm}$

Figure 134: Velocity field of topology results for different rotational values. 


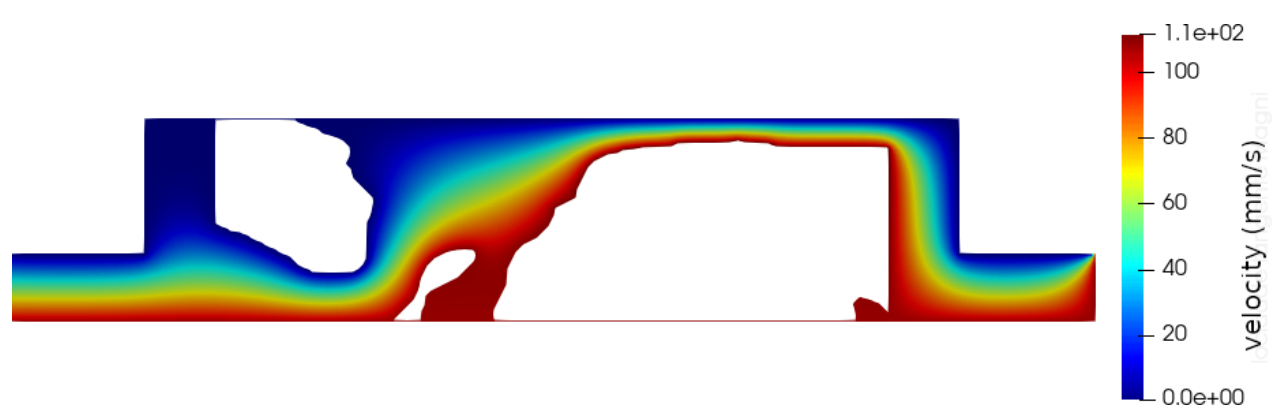

(a) Rotation of $50 \mathrm{rpm}$

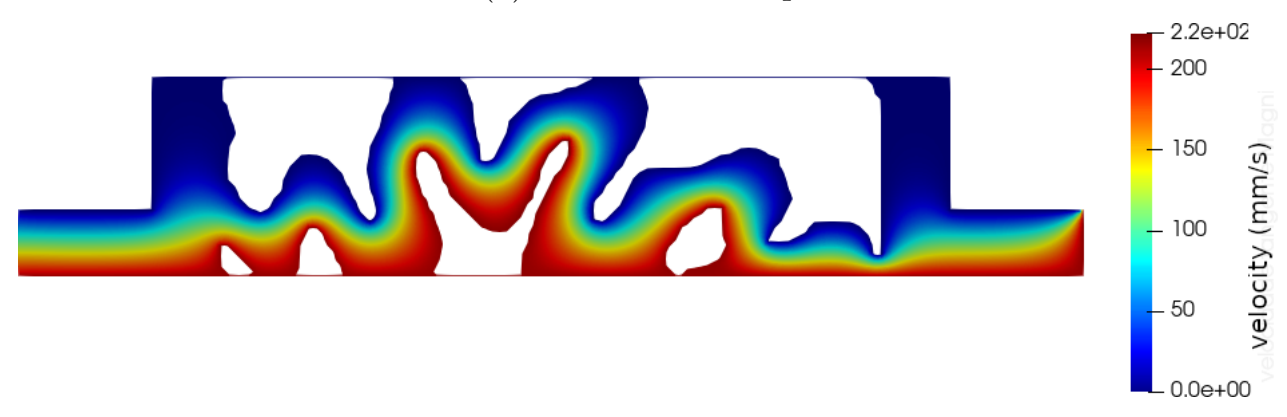

(b) Rotation of $100 \mathrm{rpm}$

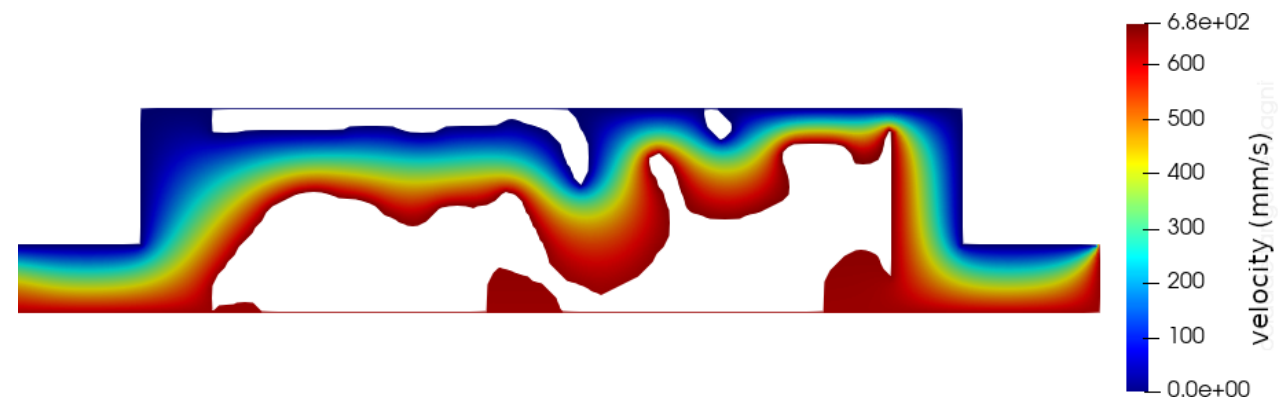

(c) Rotation of $300 \mathrm{rpm}$

Figure 135: Circumferential velocity field of topology results for different rotational values. 


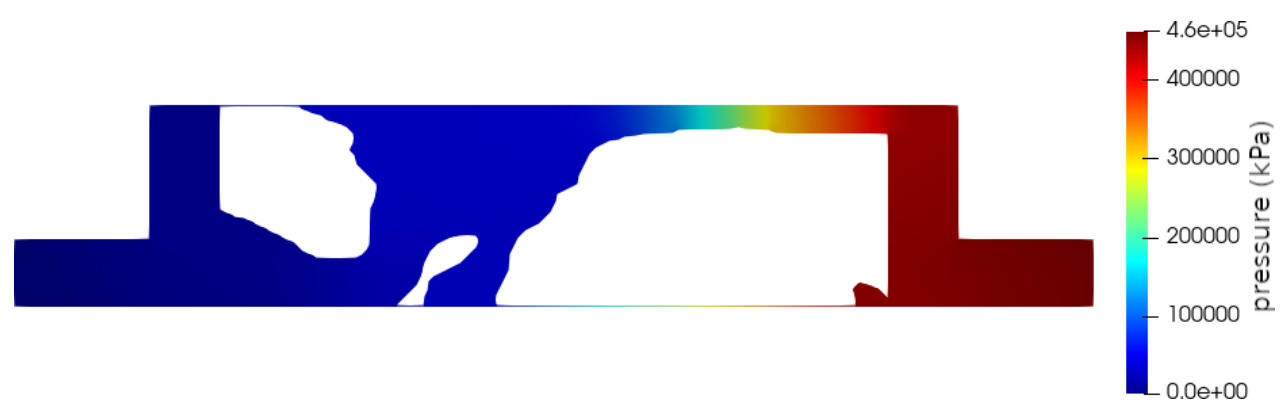

(a) Rotation of $50 \mathrm{rpm}$

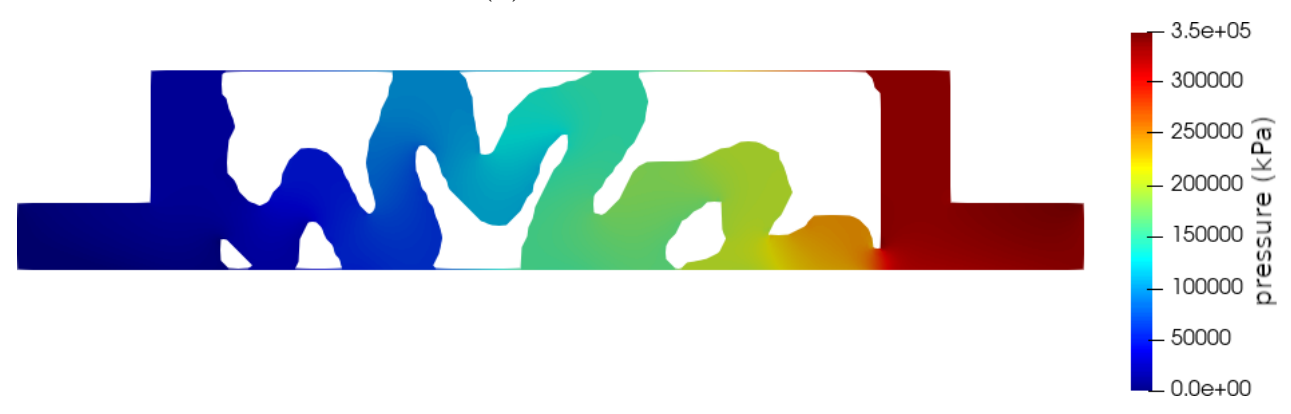

(b) Rotation of $100 \mathrm{rpm}$

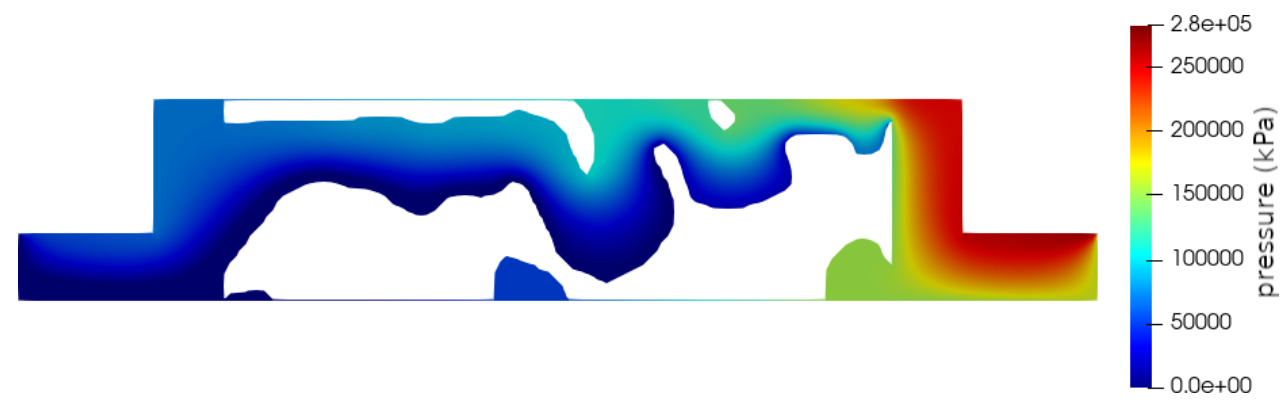

(c) Rotation of $300 \mathrm{rpm}$

Figure 136: Pressure field of topology results for different rotational values.

Table 14: Performance of optimized labyrinth seal using FSI for different rotational values.

\begin{tabular}{cl}
\hline Labyrinth seal & $\sqrt{\Delta p} / Q \times 10^{4}$ \\
\hline Baseline aspect $n=50 \mathrm{rpm}$ & 1.19 \\
Optimized aspect $n=50 \mathrm{rpm}$ & 2.64 \\
\hline Baseline aspect $n=100 \mathrm{rpm}$ & 1.15 \\
Optimized aspect $n=100 \mathrm{rpm}$ & 2.28 \\
\hline Baseline aspect $n=300 \mathrm{rpm}$ & 0.96 \\
Optimized aspect $n=300 \mathrm{rpm}$ & 1.79 \\
\hline
\end{tabular}




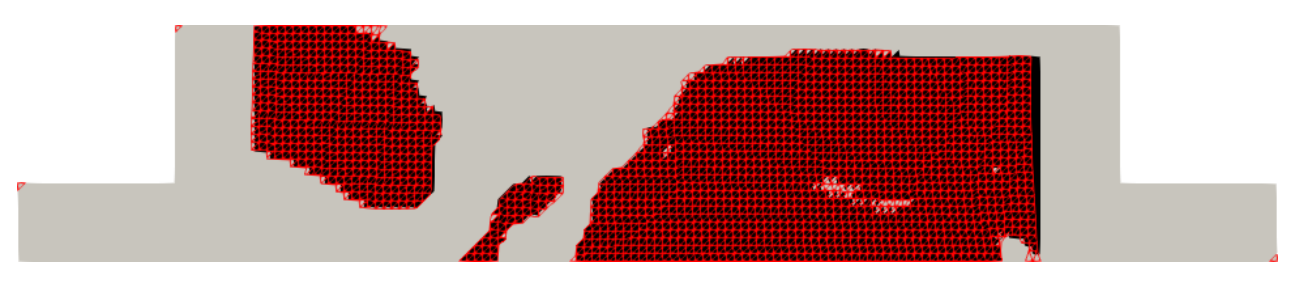

(a) Rotation of $50 \mathrm{rpm}$

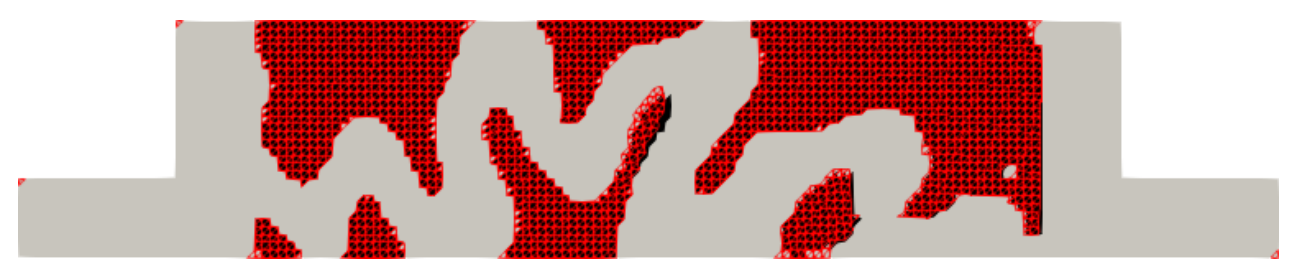

(b) Rotation of $100 \mathrm{rpm}$

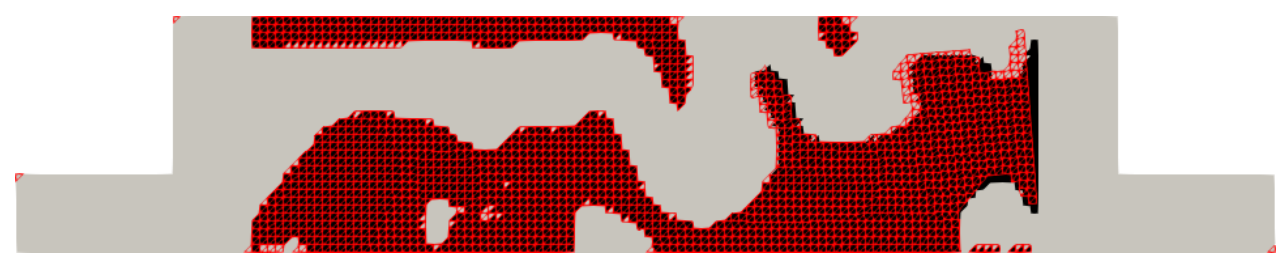

(c) Rotation of $300 \mathrm{rpm}$

Figure 137: Deformation of topology results for different rotational values.

The case of rotation equals to zero may be interesting to analyse, however structural parameter has to be changed to $W_{E}=10^{-30}$, because in this case structural terms plays an important role in the objective function and the solution seems similar to a truss and closes the flow passage. Thus, the result of a non-rotating seal can be seen in Figure 138. Since circumferential velocity is null, only the plane velocity is shown in Figure 139. Pressure field is shown in Figure 140 and displacement is shown in Figure 141. The performance values of this simulation are shown in Table 15.

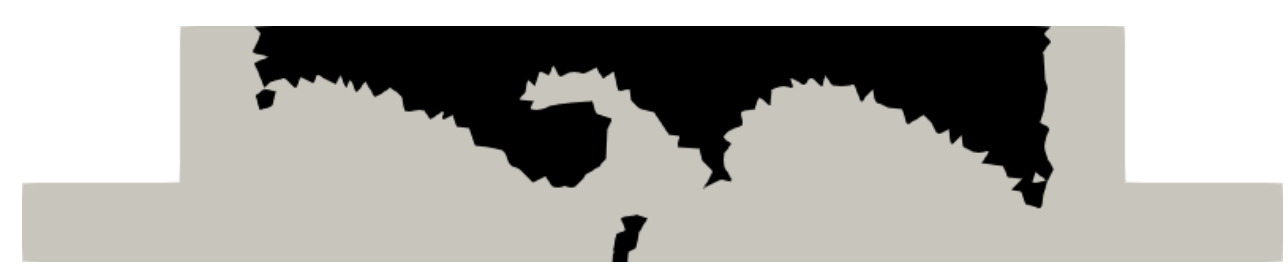

Figure 138: Labyrinth seal with null circumferential velocity. 


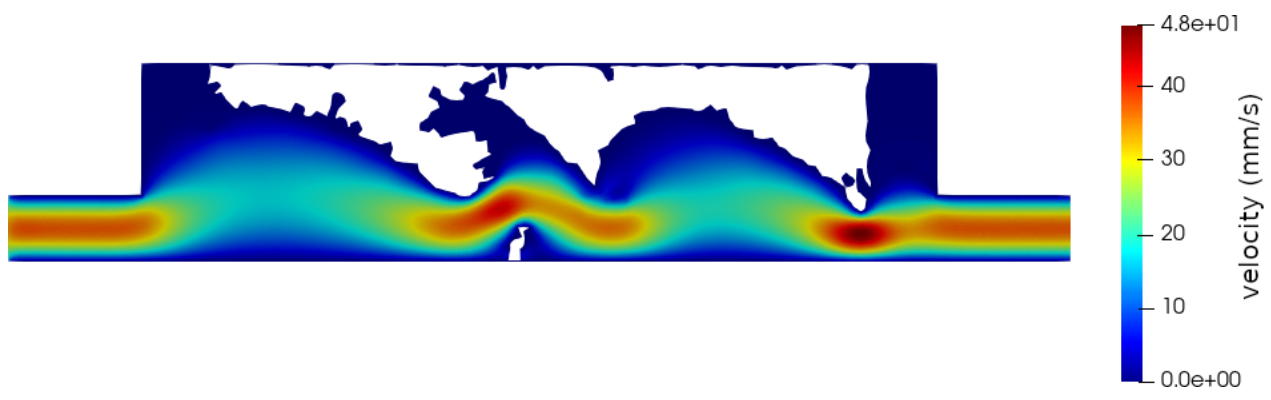

Figure 139: Modulus of plane velocity in the Labyrinth seal with null circumferential velocity.

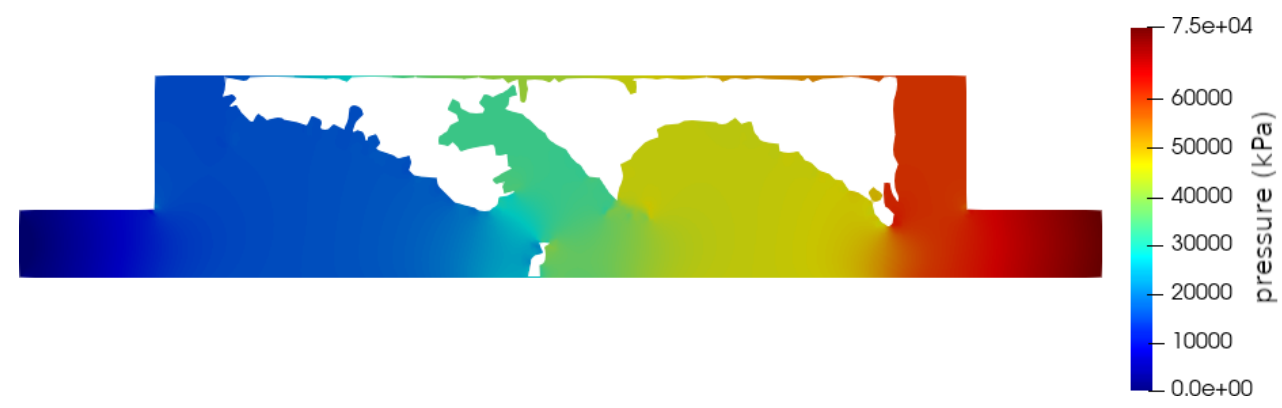

Figure 140: Pressure field in the Labyrinth seal with null circumferential velocity.

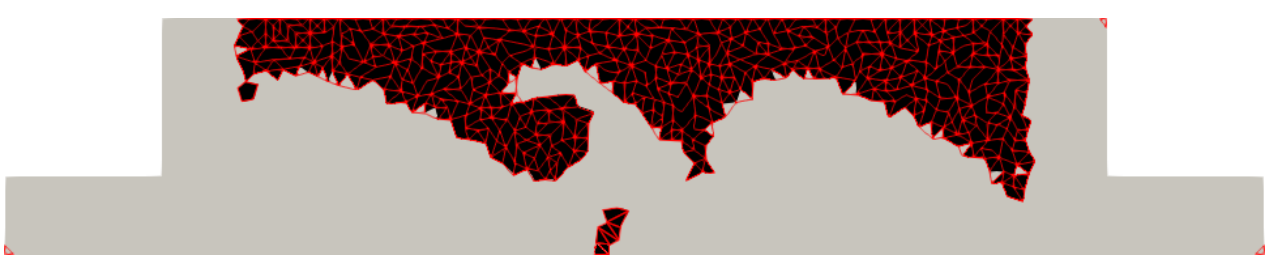

Figure 141: Displacement in red due to flow in Labyrinth seal with null circumferential velocity.

Table 15: Performance of optimized labyrinth seal using FSI for different rotational values.

\begin{tabular}{cc}
\hline Labyrinth seal & $\sqrt{\Delta p} / Q \times 10^{4}$ \\
\hline Baseline aspect $n=0 \mathrm{rpm}$ & 1.20 \\
Optimized aspect $n=0 \mathrm{rpm}$ & 3.39 \\
\hline
\end{tabular}

\subsubsection{Volume Constraint influence}

The optimization problem have worked with a volume constraint between $50 \%$ and $80 \%$ with the problem tending to keep the $50 \%$ of the full design domain. This choice is 
made based on several simulations and it is noticed that when the fluid volume contraint has lower values, it is easy for the optimizer to close the passage, bringing more issues to be solved during optimization process. On the other hand, high fluid volume constraint values makes the efficiency of the seal to be limited.

In order to illustrate this process, Figure 142 shows a different volume constraint (70\%), keeping circumferential velocity of $100 \mathrm{rpm}$. Interesting in this case is the solid concentrating near the inlet of the flow, trying to cause energy dissipation when the velocity is still high. For a wider range of volume constraints, it would be necessary to recalibrate the terms on structural and vorticity terms and this would be an unreasonable comparison.

Plane and circumferential velocity plots are shown in Figure 143 and 144, respectively, with comparison values in Table 16. Pressure plot is shown in Figure 145. The geometry deformation caused by the fluid flow is shown in Figure 146.

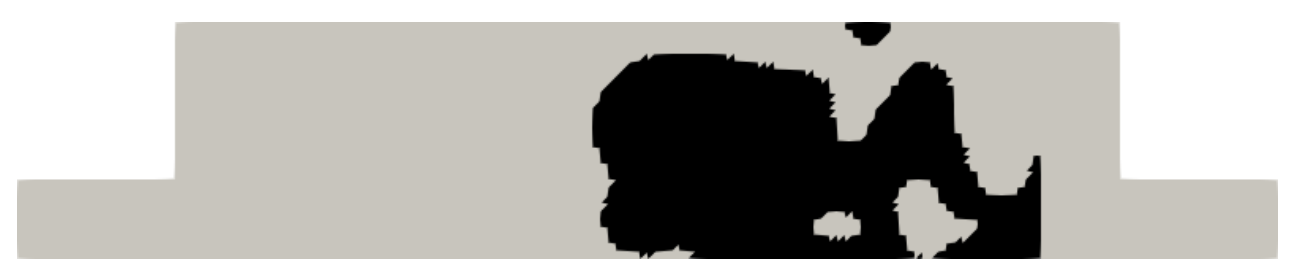

(a) Volume constraint of $70 \%$ at $100 \mathrm{rpm}$.

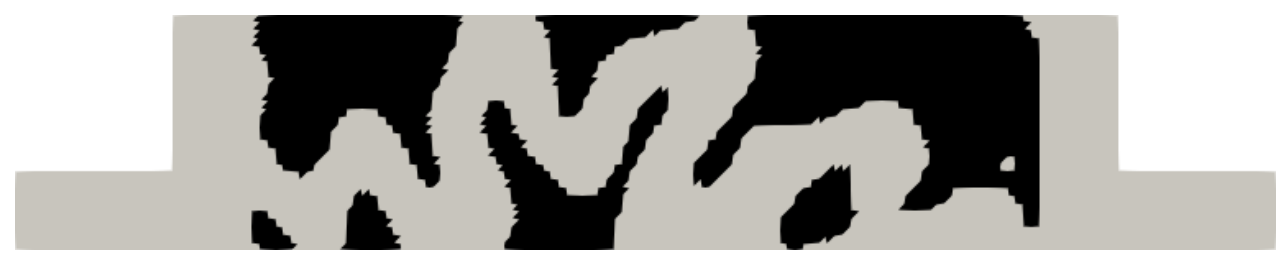

(b) Volume constraint of $50 \%$ at $100 \mathrm{rpm}$.

Figure 142: Topology results for different volume constraints. 


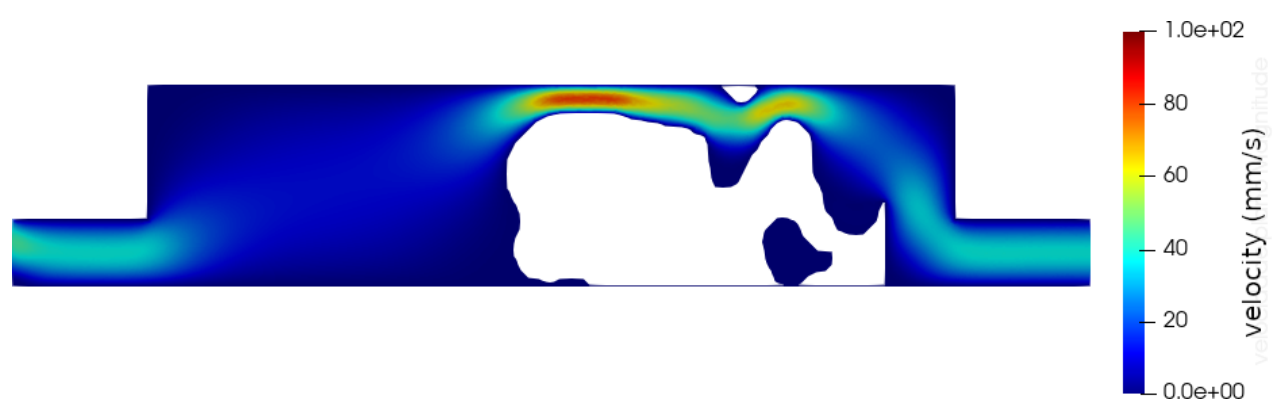

(a) Volume constraint of $70 \%$

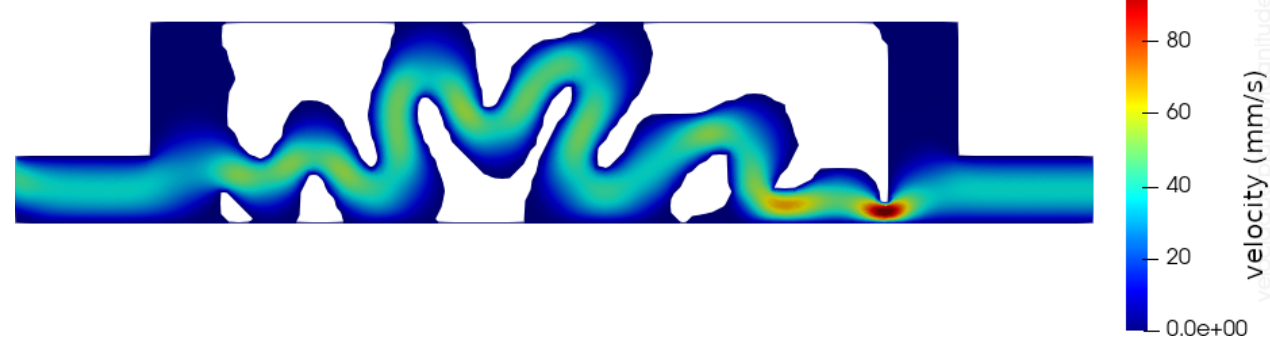

(b) Volume constraint of $50 \%$

Figure 143: Modulus of plane velocity for different topology results with different volume constraints.

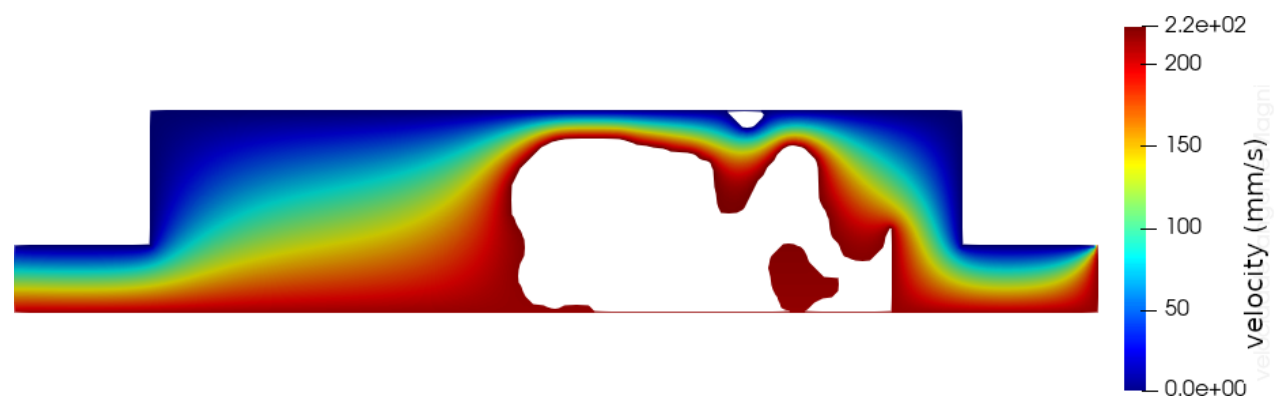

(a) Volume constraint of $70 \%$

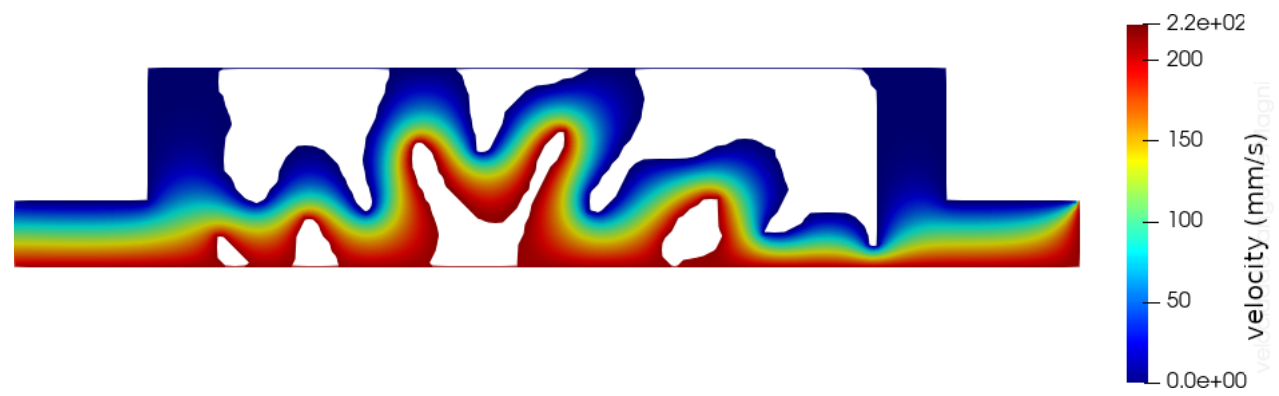

(b) Volume constraint of $50 \%$

Figure 144: Modulus of plane velocity for different topology results with different volume constraints. 


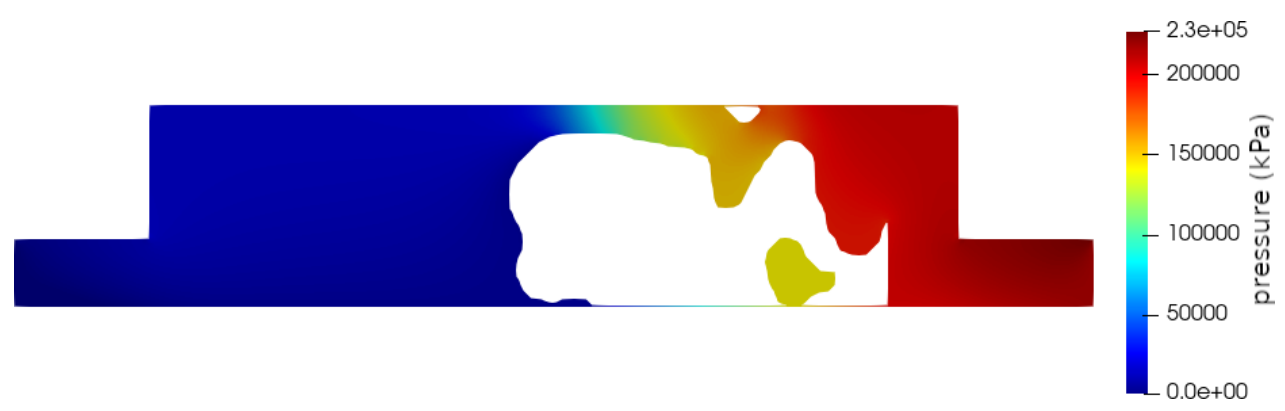

(a) Volume constraint of $70 \%$

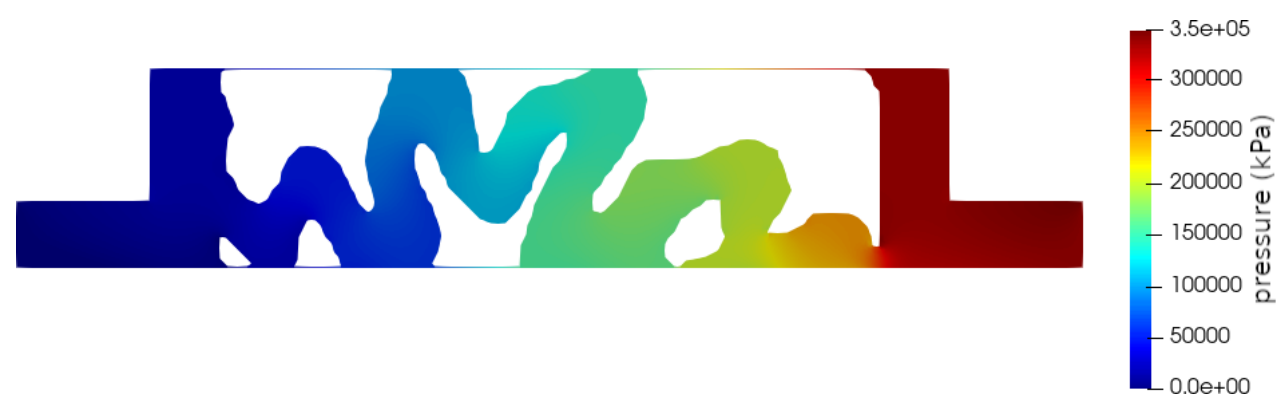

(b) Volume constraint of $50 \%$

Figure 145: Modulus of plane velocity for different topology results with different volume constraints.

Table 16: Performance of optimized labyrinth seal using FSI for different volume constraints.

\begin{tabular}{cc}
\hline Labyrinth seal & $\sqrt{\Delta p} / Q \times 10^{4}$ \\
\hline Baseline aspect $V=70 \%$ & 0.99 \\
Optimized aspect $V=70 \%$ & 1.82 \\
\hline Baseline aspect $V=50 \%$ & 1.15 \\
Optimized aspect $V=50 \%$ & 2.28 \\
\hline
\end{tabular}




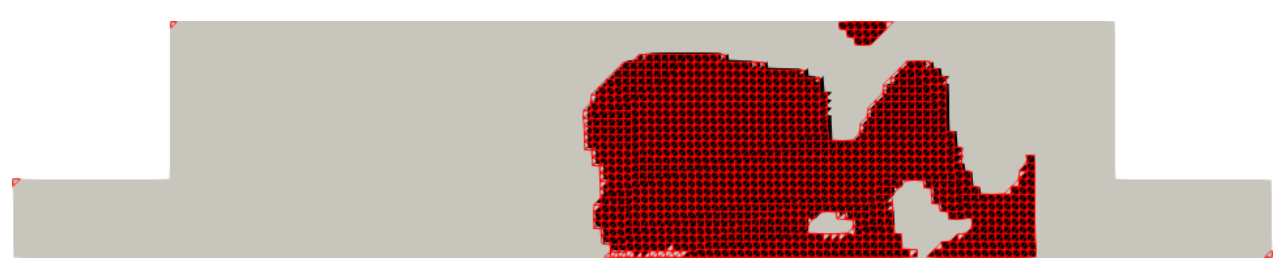

(a) Volume constraint of $70 \%$

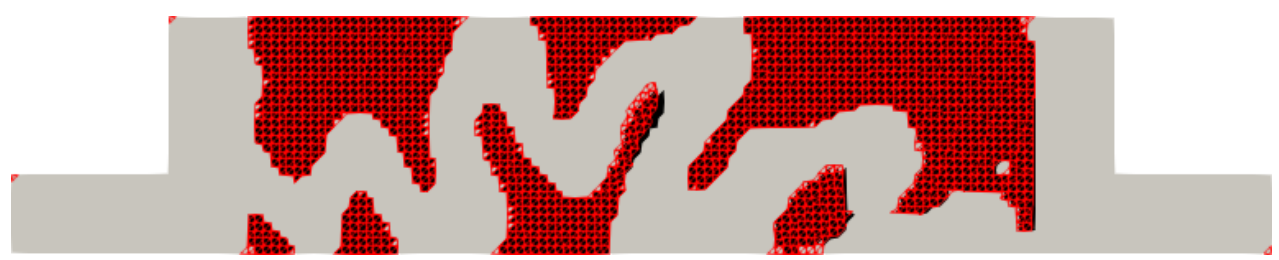

(b) Volume constraint of $50 \%$

Figure 146: Deformation of topology results for different volume constraints. 


\section{CONCLUSIONS}

This work have applied integer linear programming to design fluid diodes and labyrinth seals, using the topology optimization method with the help of open softwares, such as Fenics and Dolfin-Adjoint, together with commercial application, such as CPLEX' to deliver innovative geometries to contribute with emissions reduction challenge.

This is a pioneer work that explores how fluid diodes, more specially labyrinth seals, can be designed with the help of topology optimization method, specially with the TOBS approach. The proposed method not only helps on setting material model parameters, but also allows the design domain to have clear boundaries between fluid and solid. This behaviour during optimization process can contribute in future to more complex design optimization problem where the wall distance have to be calculated in the forward problem.

This work has expanded the application of TOBS to fluids by using sequential linear approximation of the original optimization problem in a coupled environment of FEniCS, which allows solving partial differential equations (Navier-Stokes), Dolfin-Adjoint, which allows calculating the gradient of the optimization problem, Octave which allows calculating the sensitivity vector of linearized problem, and $\mathrm{CPlex}^{\circledR}$, which is a proprietary software with a large capacity of optimizing binary variables efficiently.

Standard problems of topology optimization in fluids are studied as benchmarks to validate TOBS for fluids, such as the double pipe problem in Stokes flow, where the exact value of the objetive function is calculated, showing the proposed method is near the analytical solution and the literature. Navier-Stokes equation as forward problem is also studied and compared to literature present satisfatory results. Also, a 3D problem is simulated in order to demonstrate the easiness of expanding the proposed method.

A movable boundary condition is implemented based on a classification algorithm that uses 2D Delaunay segmentation able to detect which solid part belongs to stator or shaft. This condition allows applying the corresponding tangential velocity boundary 
condition for each case. This process can run at each optimization step automatically applying the correct boundary condition. This feature is specially beneficial to labyrinth seals, since they are divided in parts which must be attached to the shaft and parts which should be fixed with the stator.

The proposed objective function is innovative, since brings diodicity terms, recently explored in literature, influencing the fluid to flow in a certain direction, in addition to vorticity, which is not an usual term in the field of fluid optimization until this moment. Vorticity not only generates tortous fluid path, which is the primary objective of a labyrinth seal, but also brings interesting "spring" patterns in the results, which are very uncommon to topology optimization results.

Structural terms are also considered in the full optimization problem, considering the interaction caused by the pressure field into structural parts. This problem is faced as minimization of compliance of the structures and such implementation not only makes the labyrinth seal stiffer, but also eliminates the undesirable floating islands, making the devices much more feasible to manufacture.

The aspect ratio of labyrinth seals is studied and compared to a defined baseline, considering the possibility of designing only one cell, however it is shown that the shorter is the design domain, the harder is to have complex satisfatory results. The comparison of $\Delta p / \sqrt{Q}$ with the baseline confirms such difficulty.

The proposed optimization problem with all terms in objective function has a lot of local minima, making comparison with different parameters hard, however, it is possible to detect some influence according to the size of diameter (from $20 \mathrm{~mm}$ to $60 \mathrm{~mm}$ ) and rotational speed (from $50 \mathrm{rpm}$ to $300 \mathrm{rpm}$ ), possibly relating high tangential velocity with more solid material attached to the shaft. All values of pressure drop between optimized geometry and the baseline present advantages to the proposed method. Influence of volume constraint is also evaluated, showing that the algorithm tends to fill the inlet of the flow, because it may try to dissipate energy the soonest possible.

Finally, complex and efficient geometries of fluid diodes are presented using topology optimization, exploring wide range of parameters to compare to baseline devices proving the viability of the methodology used. The contribution of this work can help design engineers not only on designing non-intuitive fluid diodes, but also improving current available labyrinth seals, widely used in turbines and compressors. This improvement has high impact in equipment efficiency and also in the environment emissions, since international reports point leakage in industrial devices as one of the main causes of 
undesirable emissions.

\subsection{Future work}

As future work, the author recomends the exploration of a labyrinth seal operation with high velocity values, bringing the need of implementing turbulence models, improving the algorithm of presented work with the possibility to calculate wall distance during optimization process.

High pressure difference between two chambers can also be explored, considering compressibility analysis in flow with Mach number higher than 0.3.

Mechanical stress in labyrinth seals can also be studied, extending the structural analysis made so far in this work.

Vibration analysis is also an interesting topic when designing labyrinth seals, since vibration caused by fluid flow can influence shaft stability when reaching its ressonance values. 


\section{REFERENCES}

SÁ, L. F. N. and R. Amigo, R. C. and Novotny, A. A. and Silva, E. C. N.

ABOULAICH, R. et al. Shape optimization of labyrinth seals. International Journal of Applied Mathematics and Computer Science, v. 10, n. 2, p. 381-404, 2000.

ALLAIRE, G.; JOUVE, F. A level-set method for vibration and multiple loads structural optimization. Computer methods in applied mechanics and engineering, Elsevier, v. 194, n. 30-33, p. 3269-3290, 2005.

ALLAIRE, G.; JOUVE, F.; TOADER, A.-M. Structural optimization using sensitivity analysis and a level-set method. Journal of computational physics, Elsevier, v. 194, n. 1, p. 363-393, 2004.

ALONSO, D. H. et al. Topology optimization applied to the design of $2 \mathrm{~d}$ swirl flow devices. Structural and Multidisciplinary Optimization, Springer, v. 58, n. 6, p. 2341-2364, 2018.

ALONSO, D. H.; SAENZ, J. S. R.; SILVA, E. C. N. Non-newtonian laminar 2d swirl flow design by the topology optimization method. Structural and Multidisciplinary Optimization, Springer, p. 1-23, 2020.

AMSTUTZ, S.; ANDRÄ, H. A new algorithm for topology optimization using a level-set method. Journal of Computational Physics, Elsevier, v. 216, n. 2, p. 573-588, 2006.

ASOK, S. et al. Neural network and cfd-based optimisation of square cavity and curved cavity static labyrinth seals. Tribology International, Elsevier, v. 40, n. 7, p. 1204-1216, 2007.

BASKHARONE, E.; GHALI, A. Theoretical vs experimental rotordynamic coefficients of incompressible flow labyrinth seals. Journal of Propulsion and Power, v. 10, n. 5, p. 721-728, 1994.

BECKERS, M. Topology optimization using a dual method with discrete variables. Structural and Multidisciplinary Optimization, v. 17, p. 14-24, 1999.

BELLAOUAR, A.; KOPEY, B.; ABDELBAKI, N. Methods of the rational choice of a labyrinth seal design for gas pumping units. 2013.

BELYTSCHKO, T.; XIAO, S.; PARIMI, C. Topology optimization with implicit functions and regularization. International Journal for Numerical Methods in Engineering, Wiley Online Library, v. 57, n. 8, p. 1177-1196, 2003. 
BENDSØE, M. P.; KIKUCHI, N. Generating optimal topologies in structural design using a homogenization method. Computer methods in applied mechanics and engineering, Elsevier, v. 71, n. 2, p. 197-224, 1988.

BENDSØE, M. P.; SIGMUND, O. Material interpolation schemes in topology optimization. Archive of applied mechanics, Springer, v. 69, n. 9-10, p. 635-654, 1999.

BENDSOE, M. P.; SIGMUND, O. Topology optimization: theory, methods, and applications. [S.1.]: Springer Science \& Business Media, 2013.

BORRVALL, T.; PETERSSON, J. Topology optimization of fluids in stokes flow. International journal for numerical methods in fluids, Wiley Online Library, v. 41, n. 1, p. $77-107,2003$.

BOURDIN, B. Filters in topology optimization. International Journal for Numerical Methods in Engineering, Wiley Online Library, v. 50, n. 9, p. 2143-2158, 2001.

BOYCE, M. P. Gas turbine engineering handbook. [S.l.]: Elsevier, 2011.

BRUNS, T. E.; TORTORELLI, D. A. Topology optimization of non-linear elastic structures and compliant mechanisms. Computer methods in applied mechanics and engineering, Elsevier, v. 190, n. 26-27, p. 3443-3459, 2001.

BURGER, M.; HACKL, B.; RING, W. Incorporating topological derivatives into level set methods. Journal of Computational Physics, Elsevier, v. 194, n. 1, p. 344-362, 2004.

CHALLIS, V. J.; GUEST, J. K. Level set topology optimization of fluids in stokes flow. International journal for numerical methods in engineering, Wiley Online Library, v. 79, n. 10, p. 1284-1308, 2009.

CHANG, S.-Y.; YOUN, S.-K. Material cloud method for topology optimization. International journal for numerical methods in engineering, Wiley Online Library, v. 65, n. 10, p. 1585-1607, 2006.

CHIEN, K.-H. et al. A novel design of pulsating heat pipe with fewer turns applicable to all orientations. International journal of heat and mass transfer, Elsevier, v. 55, n. 21-22, p. 5722-5728, 2012.

CHILDS, D.; SCHARRER, J. Theory versus experiment for the rotordynamic coefficient of labyrinth gas seals: Part ii - a comparison to experiment. Journal of Vibration, Acoustics, Stress, and Reliability in Design, American Society of Mechanical Engineers, v. 110, n. 3, p. 281-287, 1988.

CHOLASEUK, D.; SRINIVASAN, V.; MODI, V. Shape optimization for fluid flow problems using bezier curves and designed numerical experiments. In: Proceedings of the 1999 ASME Design Engineering Technical Conferences. [S.l.: s.n.], 1999. p. 12-15.

DEMKO, J.; MORRISON, G.; RHODE, D. Effect of shaft rotation on the incompressible flow in a labyrinth seal. Journal of Propulsion and Power, v. 6, n. 2, p. 171-176, 1990.

DEMKO, J. A.; MORRISON, G. L.; RHODE, D. L. The prediction and measurement of incompressible flow in a labyrinth seal. Journal of engineering for gas turbines and power, American Society of Mechanical Engineers, v. 111, n. 4, p. 697-702, 1989. 
DENECKE, J. et al. Influence of rub-grooves on labyrinth seal leakage. In: AMERICAN SOCIETY OF MECHANICAL ENGINEERS. ASME Turbo Expo 2002: Power for Land, Sea, and Air. [S.l.], 2002. p. 771-779.

DILGEN, C. B. et al. Topology optimization of turbulent flows. Computer Methods in Applied Mechanics and Engineering, v. 331, p. 363 - 393, 2018. ISSN 0045-7825.

EPA, A. Inventory of us greenhouse gas emissions and sinks: 1990-2017. Environmental Protection Agency2020, 2019.

ESCHENAUER, H. A.; KOBELEV, V. V.; SCHUMACHER, A. Bubble method for topology and shape optimization of structures. Structural optimization, Springer, v. 8, n. 1, p. 42-51, 1994.

EVGRAFOV, A. The limits of porous materials in the topology optimization of stokes flows. Applied Mathematics and Optimization, Springer, v. 52, n. 3, p. 263-277, 2005.

EVGRAFOV, A. Topology optimization of slightly compressible fluids. ZAMM-Journal of Applied Mathematics and Mechanics/Zeitschrift für Angewandte Mathematik und Mechanik, Wiley Online Library, v. 86, n. 1, p. 46-62, 2006.

FOX, R. W.; MCDONALD, A. T. Introduction to Fluid Mechanics, John WileyESons. [S.l.]: Inc., New York, 1994.

FUNKE, S. W. The automation of PDE-constrained optimisation and its applications. Tese (Doutorado) - Department of Earth Science and Engineering, Imperial College London, 2013.

GERSBORG-HANSEN, A.; SIGMUND, O.; HABER, R. B. Topology optimization of channel flow problems. Structural and Multidisciplinary Optimization, Springer, v. 30, n. 3, p. 181-192, 2005.

GUEST, J. K.; GENUT, L. C. S. Reducing dimensionality in topology optimization using adaptive design variable fields. International journal for numerical methods in engineering, Wiley Online Library, v. 81, n. 8, p. 1019-1045, 2010.

GUO, X.; GU, Y. X. A new density-stiffness interpolation scheme for topology optimization of continuum structures. Engineering Computations, Emerald Group Publishing Limited, v. 21, n. 1, p. 9-22, 2004.

GUO, Y. et al. Design applicable 3d microfluidic functional units using 2d topology optimization with length scale constraints. Micromachines, Multidisciplinary Digital Publishing Institute, v. 11, n. 6, p. 613, 2020.

HABER, R. B.; JOG, C. S.; BENDSØE, M. P. A new approach to variable-topology shape design using a constraint on perimeter. Structural optimization, Springer, v. 11, n. $1-2$, p. $1-12,1996$.

HEIDRICH, F. Water flooded single screw (ssp) compressor technology. 1996.

HENDRICKS, R. C. et al. Relative performance comparison between baseline labyrinth and dual brush compressor discharge seals in a t-700 engine test. In: AMERICAN SOCIETY OF MECHANICAL ENGINEERS. ASME 1994 International Gas Turbine and Aeroengine Congress and Exposition. [S.l.], 1994. p. V001T01A095-V001T01A095. 
HESLER, S. Advanced steam labyrinth seal design. In: Phase 1 Initial Concept Evaluation EPRI - Electric Power Research Institute. [S.l.: s.n.], 2005.

HODKINSON, B. Estimation of the leakage through a labyrinth gland. Proceedings of the Institution of Mechanical Engineers, SAGE Publications Sage UK: London, England, v. 141, n. 1, p. 283-288, 1939.

KEARTON, W.; KEH, T. Leakage of air through labyrinth glands of staggered type. Proceedings of the Institution of Mechanical Engineers, SAGE Publications Sage UK: London, England, v. 166, n. 1, p. 180-195, 1952.

KHANDEKAR, S.; DOLLINGER, N.; GROLL, M. Understanding operational regimes of closed loop pulsating heat pipes: an experimental study. Applied Thermal Engineering, Elsevier, v. 23, n. 6, p. 707-719, 2003.

KHANDEKAR, S.; GROLL, M. An insight into thermo-hydrodynamic coupling in closed loop pulsating heat pipes. International journal of thermal sciences, Elsevier, v. 43, n. 1, p. 13-20, 2004.

KIM, H. et al. Improving efficiency of evolutionary structural optimization by implementing fixed grid mesh. Structural and Multidisciplinary Optimization, Springer, v. 24, n. 6 , p. 441-448, 2002.

KIM, T. S.; CHA, K. S. Comparative analysis of the influence of labyrinth seal configuration on leakage behavior. Journal of Mechanical Science and Technology, Springer, v. 23, n. 10, p. 2830, 2009.

KIM, T. S.; KANG, S. Y. Investigation of leakage characteristics of straight and stepped labyrinth seals. International Journal of Fluid Machinery and Systems, Turbomachinery Society of Japan, Korean Fluid Machinery Association, Chinese Society of Engineering Thermophysics, IAHR, v. 3, n. 3, p. 253-259, 2010.

KIM, T. S.; KANG, Y.; MOON, H. K. Aerodynamic performance of double-sided labyrinth seals. In: Fluid Machinery and Fluid Mechanics. [S.1.]: Springer, 2009. p. $377-382$.

KOMOTORI, K. Leakage characteristics of labyrinth seals with high rotating speed. In: Proc. of 1977 Tokyo Joint Gas Turbine Congress, ASME-JSME-GTSJ. [S.l.: s.n.], 1977. v. 45, p. 371.

LESCHZINER, M.; DIMITRIADIS, K. Computation of three-dimensional turbulent flow in non-orthogonal junctions by a branch-coupling method. Computers $\&$ fluids, Elsevier, v. 17, n. 2, p. 371-396, 1989.

LIN, S. et al. Topology optimization of fixed-geometry fluid diodes. Journal of Mechanical Design, American Society of Mechanical Engineers, v. 137, n. 8, p. 081402, 2015.

LIN, Z. et al. Investigation and improvement of the staggered labyrinth seal. Chinese Journal of Mechanical Engineering, Springer, v. 28, n. 2, p. 402-408, 2014.

MCGETTIGAN, M. Ireland National Inventory Report 2006: Greenhouse Gas Emissions 1990-2004 Reported to the United National Framework Convention on Climate Change. [S.1.]: EPA, 2006. 
MEYER, C.; LOWRIE, J. The leakage through straight and slant labyrinth and honeycomb seals. ASME Journal of Engineering for Power, v. 97, p. 495-502, 1974.

MORRISON, G.; CHI, D. Incompressible flow in stepped labyrinth seals. 1985.

MORRISON, G. et al. Labyrinth seals for incompressible flow. Final Report for NASA contract NAS8-34536, 1983.

OLESEN, L. H.; OKKELS, F.; BRUUS, H. A high-level programming-language implementation of topology optimization applied to steady-state navier-stokes flow. International Journal for Numerical Methods in Engineering, Wiley Online Library, v. 65 , n. 7 , p. 975-1001, 2006.

PARK, K.-S.; YOUN, S.-K. Topology optimization of shell structures using adaptive inner-front (aif) level set method. Structural and Multidisciplinary Optimization, Springer, v. 36, n. 1, p. 43-58, 2008.

PETERSSON, J.; SIGMUND, O. Slope constrained topology optimization. International Journal for Numerical Methods in Engineering, Wiley Online Library, v. 41, n. 8, p. 1417-1434, 1998.

PFAU, A. et al. Unsteady flow interactions within the inlet cavity of a turbine rotor tip labyrinth seal. Journal of Turbomachinery, American Society of Mechanical Engineers, v. 127, n. 4, p. 679-688, 2005.

PINGEN, G.; MAUTE, K. Optimal design for non-newtonian flows using a topology optimization approach. Computers $\&$ Mathematics with Applications, Elsevier, v. 59, n. 7, p. 2340-2350, 2010.

QUERIN, O.; STEVEN, G.; XIE, Y. Evolutionary structural optimisation (eso) using a bidirectional algorithm. Engineering computations, MCB UP Ltd, v. 15, n. 8, p. 1031-1048, 1998.

RHODE, D. L.; ADAMS, R. G. Computed effect of rub-groove size on stepped labyrinth seal performance. In: AMERICAN SOCIETY OF MECHANICAL ENGINEERS. ASME Turbo Expo 2000: Power for Land, Sea, and Air. [S.l.], 2000. p. V003T01A093-V003T01A093.

ROSS, S. L.; BECKINGER, R. F. et al. Compressor seal selection and justification. In: TEXAS A\&M UNIVERSITY. TURBOMACHINERY LABORATORIES. Proceedings of the 32nd Turbomachinery Symposium. [S.l.], 2003.

ROZVANY, G. I.; ZHOU, M.; BIRKER, T. Generalized shape optimization without homogenization. Structural optimization, Springer, v. 4, n. 3-4, p. 250-252, 1992.

SÁ, L. et al. Topological derivatives applied to fluid flow channel design optimization problems. Structural and Multidisciplinary Optimization, Springer, v. 54, n. 2, p. 249-264, 2016.

SÁ, L. F. N. et al. Topological derivatives applied to fluid flow channel design optimization problems. Structural and Multidisciplinary Optimization, n. 2003, feb 2016. ISSN 1615-147X. 
SÁ, L. F. N. et al. Topology optimization of turbulent rotating flows using spalart-allmaras model. Computer Methods in Applied Mechanics and Engineering, v. 373, p. 113551, 2021. ISSN 0045-7825.

SATO, Y. et al. Topology optimization of a no-moving-part valve incorporating pareto frontier exploration. Structural and Multidisciplinary Optimization, Springer, v. 56, n. 4, p. 839-851, 2017.

SCHRAMM, V. et al. Shape optimization of a labyrinth seal applying the simulated annealing method. International Journal of Rotating Machinery, Hindawi, v. 10, n. 5, p. 365-371, 2004.

SCHRAMM, V. et al. Influence of a honeycomb facing on the flow through a stepped labyrinth seal. In: AMERICAN SOCIETY OF MECHANICAL ENGINEERS. ASME Turbo Expo 2000: Power for Land, Sea, and Air. [S.l.], 2000. p. V003T01A092V003T01A092.

SETHIAN, J. A.; WIEGMANN, A. Structural boundary design via level set and immersed interface methods. Journal of computational physics, Elsevier, v. 163, n. 2, p. 489-528, 2000.

SHAHPAR, S. A comparative study of optimisation methods for aerodynamic design of turbomachinery blades. In: AMERICAN SOCIETY OF MECHANICAL ENGINEERS. ASME Turbo Expo 2000: Power for Land, Sea, and Air. [S.l.], 2000. p. V001T03A087-V001T03A087.

SIGMUND, O.; PETERSSON, J. Numerical instabilities in topology optimization: a survey on procedures dealing with checkerboards, mesh-dependencies and local minima. Structural optimization, Springer, v. 16, n. 1, p. 68-75, 1998.

SIVAPURAM, R.; PICELLI, R. Topology optimization of binary structures using integer linear programming. Finite Elements in Analysis and Design, Elsevier, v. 139, p. 49-61, 2018.

SIVAPURAM, R.; PICELLI, R.; XIE, Y. M. Topology optimization of binary microstructures involving various non-volume constraints. Computational Materials Science, Elsevier, v. 154, p. 405-425, 2018.

SOUZA, B. et al. Topology optimization of fluid flow by using integer linear programming. Structural and Multidisciplinary Optimization, Springer, p. 1-20, 2021.

STOCKER, H. Advanced labyrinth seal design performance for high pressure ratio gas turbines. In: AMERICAN SOCIETY OF MECHANICAL ENGINEERS. ASME 1975 Winter Annual Meeting: GT Papers. [S.l.], 1975. p. V001T01A005-V001T01A005.

STOCKER, H. Determining and improving labyrinth seal performance in current and advanced high performance gas turbines. AGARD Seal Technol. in Gas Turbine Eng. 22 p(SEE N 79-11056 02-07), 1978.

SUBRAMANIAN, S.; SEKHAR, A.; PRASAD, B. Influence of combined radial location and growth on the leakage performance of a rotating labyrinth gas turbine seal. Journal of Mechanical Science and Technology, Springer, v. 29, n. 6, p. 2535-2545, 2015. 
SVANBERG, K.; WERNE, M. Topology optimization by sequential integer linear programming. The Netherlands, Dordrecht: Springer, 2006.

TAYLOR, C.; HOOD, P. A numerical solution of the navier-stokes equations using the finite element technique. Computers \& Fluids, Elsevier, v. 1, n. 1, p. 73-100, 1973.

TIPTON, D. Labyrinth seal analysis (analytical and experimental development of a design model for labyrinth seals). Technical Report of Air Force Wright Aeronautical Laboratories, v. 3, p. 1-309, 1986.

VAKILI, A. D. et al. Advanced labyrinth seals for steam turbine generators. In: AMERICAN SOCIETY OF MECHANICAL ENGINEERS. ASME Turbo Expo 2006: Power for Land, Sea, and Air. [S.l.], 2006. p. 1599-1608.

VANCE, J. M.; ZIERER, J.; CONWAY, E. Effect of straight through labyrinth seals on rotordynamics. ASME, NEW YORK, NY(USA)., v. 60, p. 159-171, 1993.

VERMES, G. A fluid mechanics approach to the labyrinth seal leakage problem. Journal of engineering for power, American Society of Mechanical Engineers, v. 83, n. 2, p. 161-169, 1961.

VRIES, S. de et al. Design and operation of a tesla-type valve for pulsating heat pipes. International Journal of Heat and Mass Transfer, Elsevier, v. 105, p. 1-11, 2017.

WANG, M. Y.; WANG, X.; GUO, D. A level set method for structural topology optimization. Computer methods in applied mechanics and engineering, Elsevier, v. 192, n. 1-2, p. 227-246, 2003.

WANG, W.-z. et al. Numerical analysis of leakage flow through two labyrinth seals1. Journal of Hydrodynamics, Ser. B, Elsevier, v. 19, n. 1, p. 107-112, 2007.

WANG, X.; WANG, M.; GUO, D. Structural shape and topology optimization in a level-set-based framework of region representation. Structural and Multidisciplinary Optimization, Springer, v. 27, n. 1-2, p. 1-19, 2004.

WASCHKA, W. et al. Heat transfer and leakage in high-speed rotating stepped labyrinth seals. AGARD, Heat Transfer and Cooling in Gas Turbines 10 p(SEE N 93-29926 11-07), 1993.

WITTIG, S. et al. Numerical predictions and measurements of discharge coefficients in labyrinth seals. In: AMERICAN SOCIETY OF MECHANICAL ENGINEERS. ASME 1987 International Gas Turbine Conference and Exhibition. [S.l.], 1987. p. V001T01A064-V001T01A064.

WRÓBLEWSKI, W. et al. Optimization of tip seal with honeycomb land in lp counter rotating gas turbine engine. Task Quarterly, v. 14, n. 3, p. 189-207, 2010.

WYLER, J. Design and testing of a new double labyrinth seal. In: American Society of Mechanical Engineers and American Society of Lubrication Engineers, Joint Lubrication Conference, New Orleans, LA. [S.1.: s.n.], 1981. p. 1981.

XIE, Y.; STEVEN, G. P. Optimal design of multiple load case structures using an evolutionary procedure. Engineering computations, MCB UP Ltd, v. 11, n. 4, p. 295-302, 1994. 
XIE, Y. M.; STEVEN, G. P. A simple evolutionary procedure for structural optimization. Computers \& structures, Elsevier, v. 49, n. 5, p. 885-896, 1993.

YOON, G. H. Topology optimization for turbulent flow with spalart-allmaras model. Computer Methods in Applied Mechanics and Engineering, Elsevier, v. 303, p. 288-311, 2016.

YOON, G. H.; KIM, Y. Y. Element connectivity parameterization for topology optimization of geometrically nonlinear structures. International journal of solids and structures, Elsevier, v. 42, n. 7, p. 1983-2009, 2005.

YOUN, S.-K.; PARK, S.-H. A study on the shape extraction process in the structural topology optimization using homogenized material. Computers 85 Structures, Elsevier, v. 62 , n. 3 , p. 527-538, 1997.

ZHOU, M.; SHYY, Y.; THOMAS, H. Checkerboard and minimum member size control in topology optimization. Structural and Multidisciplinary Optimization, Springer, v. 21, n. 2 , p. $152-158,2001$.

ZHU, Y.; HE, F. Analysis of leakage characteristics of labyrinth seals. Chinese Journal of Aeronautics, v. 3, p. 233-238, 1990. 


\section{APPENDIX A - FILTER OF THE DESIGN VARIABLES}

Section 3.2.6 highlights the filtering method used in this work and more details of it is discussed here. In order to use a continous equation of filtering method, the Heaviside equation is regularized. First a weight function is defined in Equation A.1, then a proximity-based weighted average of active elements is calculated in Equation A.2. Finally, the Heaviside equation is regularized in Equation A.3.

$$
\begin{gathered}
w\left(x_{i}-x_{e}\right)=\left\{\begin{array}{c}
\frac{r_{\min }-\left\|x_{i}-x_{e}\right\|}{r_{\min }} \text { if } i \in N^{e} \\
0 \text { otherwise }
\end{array}\right. \\
\mu^{e}(\alpha)=\frac{\sum_{i \in N^{e}} \alpha_{i} w\left(x_{i}-x_{e}\right)}{\sum_{i \in N^{e}} w\left(x_{i}-x_{e}\right)} \\
\phi(\alpha)=1-e^{-\beta \mu^{e}(\alpha)}+\mu^{e}(\alpha) e^{-\beta}
\end{gathered}
$$

Also, the sensitivities of the problem should be adjusted, so the derivative of $p h i$ with respect to a single active design variable is calculated first in Equation A.4. Thus, the sensitivities of the topology optimization can be adjusted in Equation A.5.

$$
\frac{\partial \phi}{\partial \alpha_{i}}=\left(\beta e^{-\beta \mu^{e}(\alpha)}+e^{-\beta}\right) \frac{w\left(x_{i}-x_{e}\right)}{\sum_{i \in N^{e}} w\left(x_{i}-x_{e}\right)}
$$

with $\beta$ as an weight factor usually from 1 to 10 (1 is used in this work except when exposed its value). 


$$
\frac{\partial f}{\partial \alpha_{i}}=\sum_{e \in \Omega} \frac{\partial f}{\partial \phi} \frac{\partial \phi}{\partial \alpha_{i}}
$$

with $f$ as a generic objective function. 


\section{APPENDIX B - FLUID FLOW VERIFICATION}

In order to check if the implemented CFD corresponds to a correct solution, it is now compared with comercial software COMSOL 5.4.

The chosen problem is similar to the more complex analysis made in this work with a 2D swirl system. Properties used in both simulations are illustrated in Table 17, the velocity magnitude plot considering all three directions is shown in Figure 147, and pressure field in Figure 149. The analysis presents very similar values.

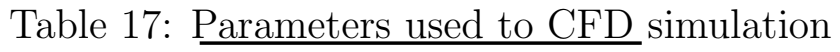

\begin{tabular}{cc} 
parameters & value \\
\hline$d$ & $40 \mathrm{~mm}$ \\
$n$ & $100 \mathrm{rpm}$ \\
$\mu$ & 1.0 \\
$R e_{\text {inlet }}$ & 200 \\
aspect ratio & $1: 4$ \\
\hline
\end{tabular}




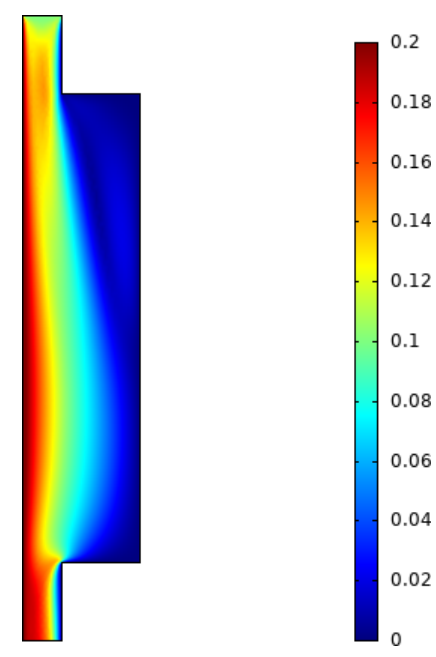

(a) Comsol

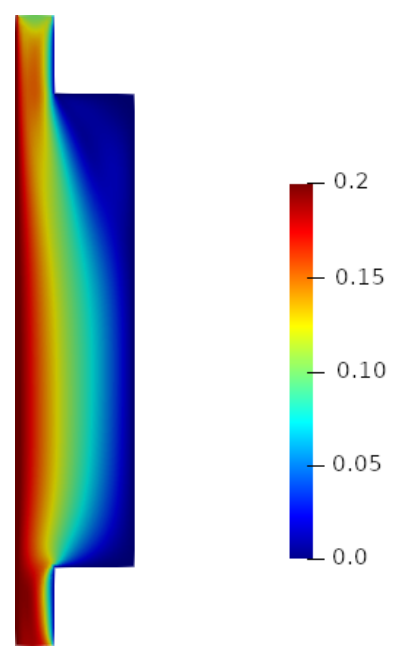

(b) Implemented code

Figure 147: Comparison in velocity plot.

In order to have a more clear comparison of velocity magnitude, the values from both solvers are performed in Figure 148. It is possible to see reasonable values even with a small distance in the middle of the design domain.

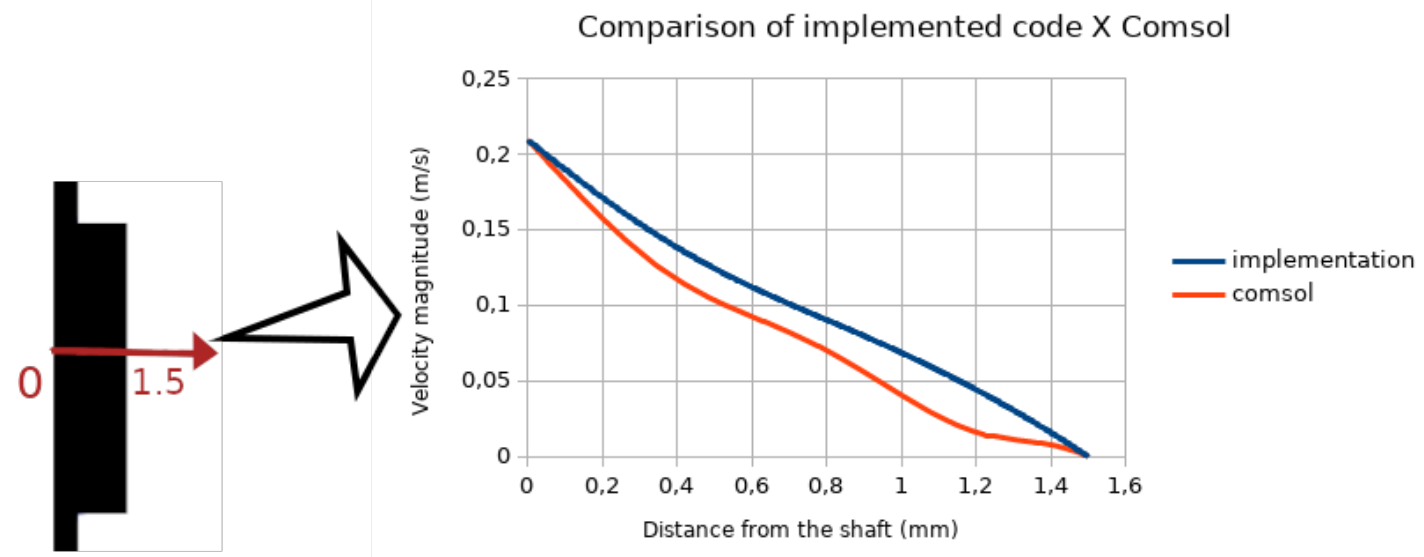

Figure 148: Velocity magnitude over line. 

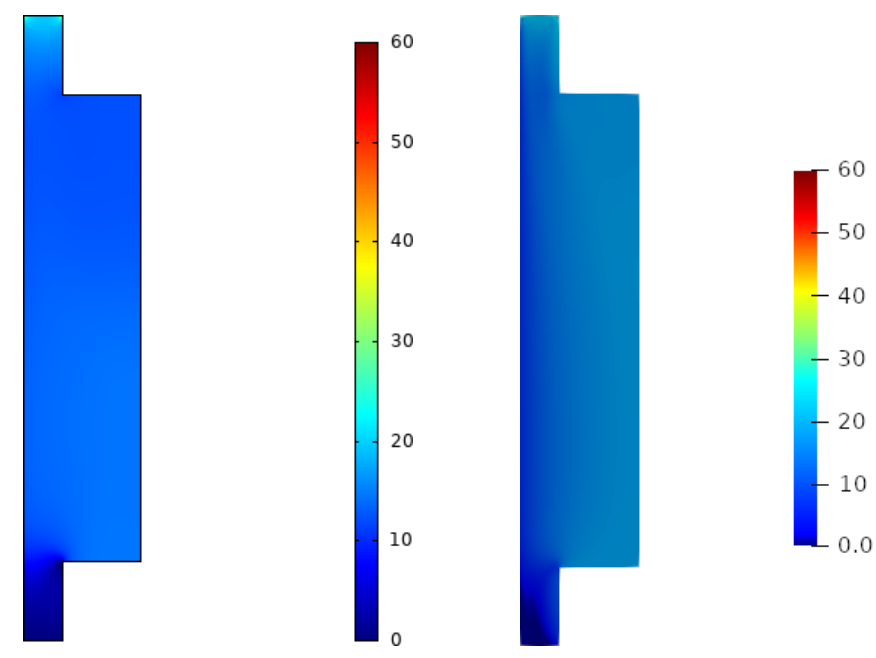

(a) Comsol

(b) Implemented code

Figure 149: Comparison in pressure plot.

There is some difference found in some details of the plots, however this occurs due to different mesh, tolerance and methods of non-linear solvers and color plot that are not from the same program. 


\section{APPENDIX C - GRADIENT VERIFICATION}

In order to check if the sensitivity is correctly calculated in this work, it is compared with a finite difference by changing $10^{-10}$ each design variable in the more complete problem (see parameters in Table 18) that takes diodicity, vorticity and fsi into consideration (Equation 3.11). Such comparision can be seen in Figure 150a-b.

Table 18: Optimization parameters used to check gradient

\begin{tabular}{cc} 
parameters & value \\
\hline$d$ & $40 \mathrm{~mm}$ \\
$n$ & $100 \mathrm{rpm}$ \\
$r_{\min }$ & $0.20 \mathrm{~mm}$ \\
$\kappa_{\max }$ & $10^{10}$ \\
$W_{F}$ & 0.1 \\
$W_{E}$ & $10^{-20}$ \\
$W_{V}$ & $2 \cdot 10^{-7}$ \\
$V_{u p}$ & $80 \%$ \\
$V_{\text {down }}$ & $50 \%$ \\
\hline
\end{tabular}

A random choice of cells is performed and they are illustrated in Figure 151 with the more detailed values in Table 19.

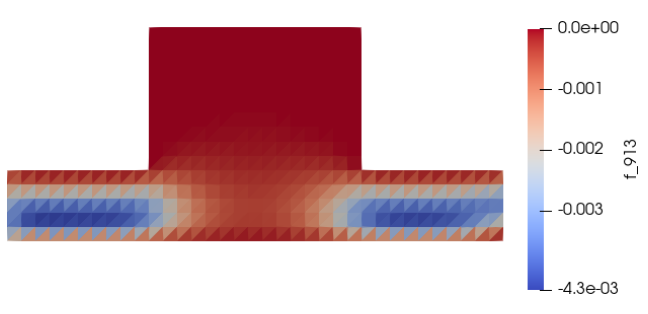

(a) Finite Difference

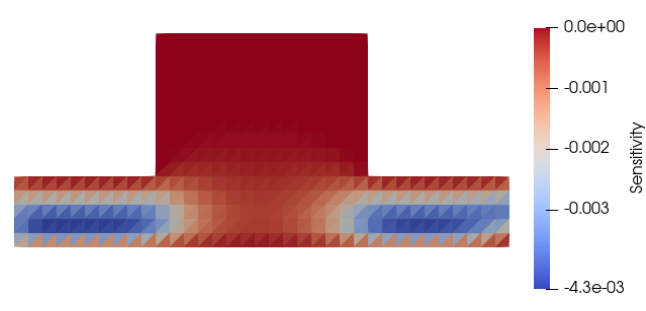

(b) Calculated Sensitivity

Figure 150: Difference of calculated sensitivity and finite difference 


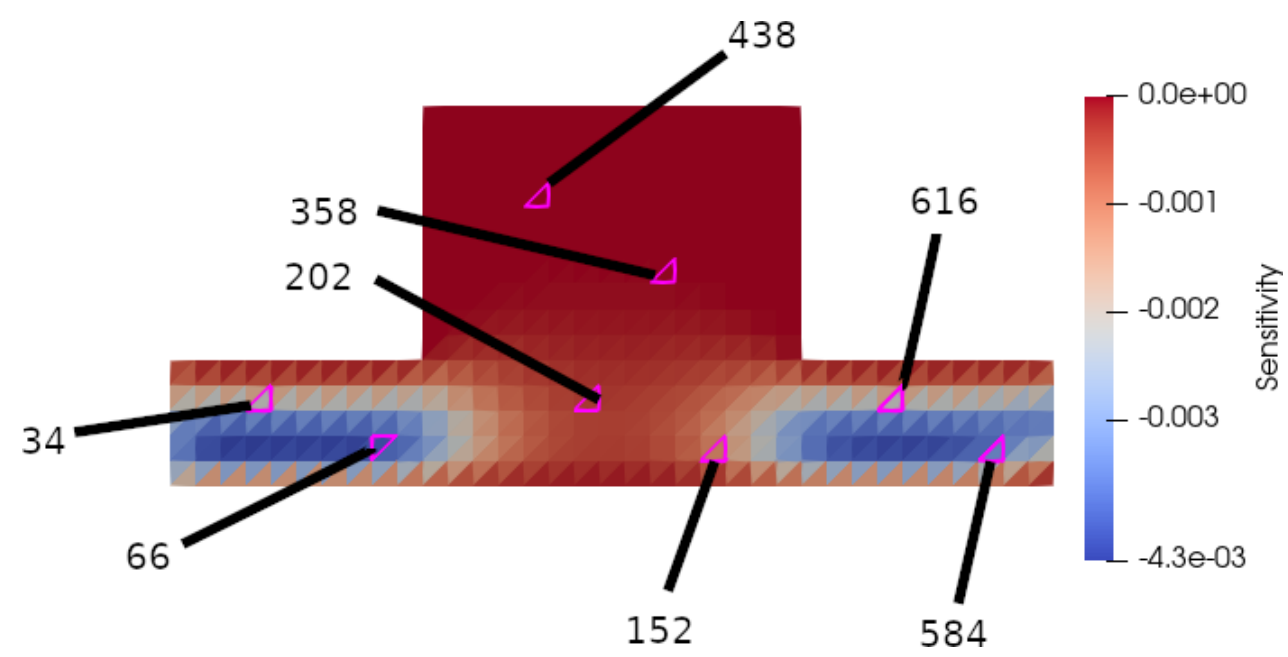

Figure 151: Identification of cell in the calculated sensitivity field.

Table 19: Parameters used in the fluid diode optimization

\begin{tabular}{cccc}
\hline Point Number & Finite Difference & Calculated Sensitivity & Relative Error(\%) \\
\hline ID:37 & $-4,088 \mathrm{E}-03$ & $-4,092 \mathrm{E}-03$ & $-0,09 \%$ \\
ID:66 & $-2,214 \mathrm{E}-03$ & $-2,213 \mathrm{E}-03$ & $0,04 \%$ \\
ID:152 & $-1,097 \mathrm{E}-03$ & $-1,096 \mathrm{E}-03$ & $0,05 \%$ \\
ID:202 & $-3,775 \mathrm{E}-04$ & $-3,759 \mathrm{E}-04$ & $0,42 \%$ \\
ID:358 & $-1,998 \mathrm{E}-05$ & $-1,908 \mathrm{E}-05$ & $4,74 \%$ \\
ID:438 & $-2,220 \mathrm{E}-06$ & $-2,255 \mathrm{E}-06$ & $-1,52 \%$ \\
ID:584 & $-3,402 \mathrm{E}-03$ & $-3,403 \mathrm{E}-03$ & $-0,04 \%$ \\
ID:616 & $-2,229 \mathrm{E}-03$ & $-2,229 \mathrm{E}-03$ & $0,00 \%$ \\
\hline
\end{tabular}

All the points have resoable values with error bellow $5 \%$. 